\title{
Fetal asphyxia : friend or foe? : the impact of fetal asphyxia and preconditioning during early periods of brain development
}

Citation for published version (APA):

Strackx, E. (2010). Fetal asphyxia : friend or foe? : the impact of fetal asphyxia and preconditioning during early periods of brain development. [Doctoral Thesis, Maastricht University]. Maastricht University. https://doi.org/10.26481/dis.20100114es

Document status and date:

Published: 01/01/2010

DOI:

10.26481/dis.20100114es

Document Version:

Publisher's PDF, also known as Version of record

Please check the document version of this publication:

- A submitted manuscript is the version of the article upon submission and before peer-review. There can be important differences between the submitted version and the official published version of record.

People interested in the research are advised to contact the author for the final version of the publication, or visit the DOI to the publisher's website.

- The final author version and the galley proof are versions of the publication after peer review.

- The final published version features the final layout of the paper including the volume, issue and page numbers.

Link to publication

\footnotetext{
General rights rights.

- You may freely distribute the URL identifying the publication in the public portal. please follow below link for the End User Agreement:

www.umlib.nl/taverne-license

Take down policy

If you believe that this document breaches copyright please contact us at:

repository@maastrichtuniversity.nl

providing details and we will investigate your claim.
}

Copyright and moral rights for the publications made accessible in the public portal are retained by the authors and/or other copyright owners and it is a condition of accessing publications that users recognise and abide by the legal requirements associated with these

- Users may download and print one copy of any publication from the public portal for the purpose of private study or research.

- You may not further distribute the material or use it for any profit-making activity or commercial gain

If the publication is distributed under the terms of Article $25 \mathrm{fa}$ of the Dutch Copyright Act, indicated by the "Taverne" license above, 
Copyright (c) Eveline Strackx, Maastricht 2010

ISBN: 978-90-9024961-2

Fetal asphyxia: Friend or foe? The impact of fetal asphyxia and preconditioning during early periods of brain development

Thesis with summary in English and Dutch

All rights reserved. No part may be reproduced or transmitted in any form or by any means, electronic or mechanical including photocopying, recording or by any information storage and retrieval system, without permission, in writing, from the copyholder.

Cover design by Ragdoll Design (Priscilla Brouwers)

Typesetting and layout: Eveline Strackx

Printed by PrintPartners Ipskamp, Enschede 


\title{
Fetal asphyxia: Friend or foe?
}

\section{The impact of fetal asphyxia and preconditioning during early periods of brain development}

\author{
Proefschrift \\ ter verkrijging van de graad van doctor \\ aan de Universiteit Maastricht, \\ op gezag van de Rector Magnificus, \\ Prof. mr. G.P.M.F. Mols, \\ volgens het besluit van het College van Decanen \\ in het openbaar te verdedigen op \\ donderdag 14 januari 2010 om 16.00 uur \\ door \\ Eveline Strackx \\ geboren op 10 oktober 1984 te Neerpelt
}




\section{Promotores}

Prof. dr. J.S.H. Vles

Prof. dr. L.J.I. Zimmermann

\section{Copromotor}

dr. A.W.D. Gavilanes

\section{Beoordelingscommisie/Evaluation committee}

Prof. dr. M. de Baets (Chairman)

Prof. dr. M. Daemen

Prof. dr. D. Giussani, University of Cambridge, UK

Prof. dr. L. Lagae, Universitair ziekenhuis Leuven

Prof. dr. F. Ramaekers 
Similia similibus curentur 'Likes are cured by likes'

Hippocrates (460-370 BC) 
Chapter 2

A combined behavioral and morphological study on the effects of fetal asphyxia on the nigrostriatal dopaminergic system

Chapter 3

Fetal asphyxia leads to a decrease in dorsal raphe serotonergic neurons

Chapter 4

Fetal asphyxia leads to the loss of striatal presynaptic boutons in adult rats

Chapter 5 Alternations of brain apoptotic cell death and carotid artery reactivity in a fetal asphyxia preconditioning model

Chapter 6

Fetal asphyctic preconditioning protects against perinatal asphyxiainduced apoptotic cell death and astrogliosis in the neonatal brain

Chapter 7

Fetal asphyctic preconditioning protects against perinatal asphyxiainduced behavioral consequences in adulthood

Chapter 8

General discussion

References

Summary 

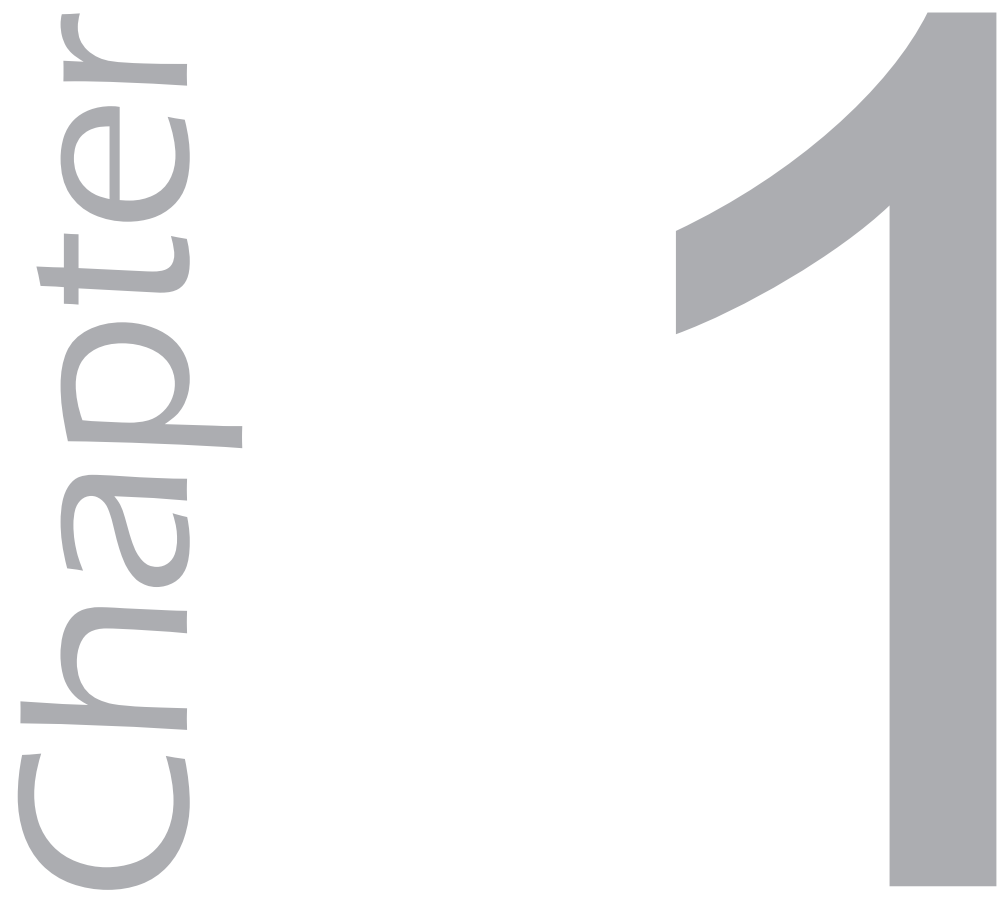

General introduction

E.Strackx 
sphyxia during development, whether pre-, peri- or postnatally, is
a world-wide problem [3]. It is a major cause of neonatal morbid-
ity and mortality, leading to roughly $23 \%$ of all neonatal deaths
(Figure 1) [3]. Approximately 15\% of all cases are considered moderate-to-severe, associated with adverse multi-organ deficits, like hepatic, heart and renal failure [6]. Brain damage is, however, of most concern, because the developing brain is extremely vulnerable and brain development determines the quality of life. The prevalence of moderate or severe post-asphyctic encephalopathy is 0.86 per 1000 term life births [7]. Moreover, the prevalence of asphyxia during birth or pregnancy in premature newborn infants is even higher [8]. The impact on the future lives of both the children and their families is substantial and therefore the clinical and the socio-economic implications are significant as well [9].

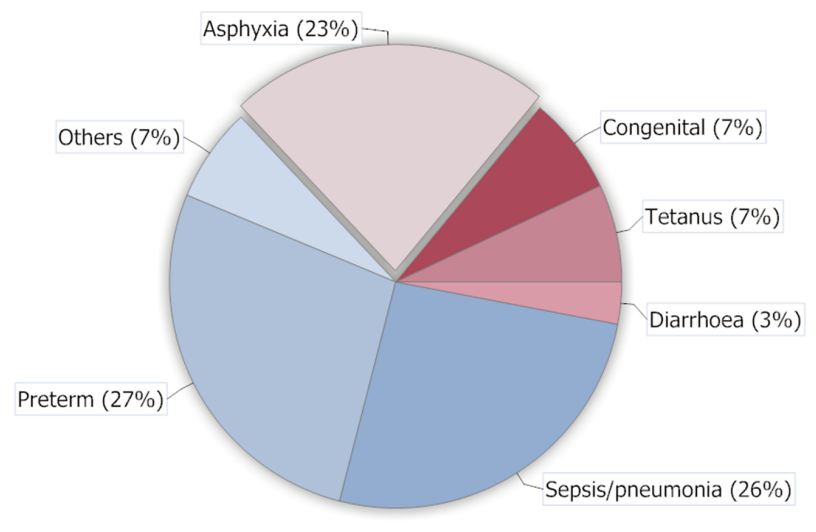

Figure 1: Estimated direct causes of neonatal deaths for the year 2000 (Adapted from Lawn et al. [3])

Over the past two centuries, doctors and scientists have tried to study the relationship between asphyxia during pregnancy/birth and permanent damage to the central nervous system (CNS). Before 1975, these studies mainly consisted of clinical and pathological non-experimental papers. The first detailed description was presented by William Little in 1861, defining a causal relationship between abnormal parturition and central nervous system (CNS) damage [10]. He noted associations between spasticity and the degree of prematurity, 
difficult labor and asphyxia with convulsions. Two decades later William Osler termed these disabilities 'cerebral palsy' [11]. In addition, he also associated birth complications with impaired intellect, speech problems and epilepsy. The first important scientific, pathophysiological studies of asphyxia were done by Brann and Myers in 1975 [12-14]. They used primates to determine the occurrence and pattern of perinatal brain damage.

Over time different definitions of asphyxia have been used. Etymologically, it comes from the Greek, meaning 'without pulse', while in the 1930s N.J. Eastman defined asphyxia as 'an inability of the child to breathe and apnea associated with neonatal acidosis' [15]. Those definitions are, however, not satisfactory. Nowadays asphyxia during development is defined in most textbooks as an impaired respiratory gas exchange between the mother and the fetus or newborn child leading to hypoxemia, accompanied with hypercapnia and metabolic and respiratory acidosis [16]. It can take place during several stages of development: prenatal, perinatal or postnatal. The most common causes of asphyxia during development are a compressed or prolapsed umbilical cord, reduced uteroplacental blood flow due to hypotension, placental abruption and other placental or uterine lesions $[16,17]$. The severity of the damage usually depends on the degree of acidosis, hypoxia and hypercarbia, comprising a number of sensitizing factors, including the developmental stage of the brain, the severity and duration of the insult, the metabolic and cardiovascular status and the intermittency of the insult [18].

It is important to note that the fetus owns a unique set of physiological and metabolic adaptive mechanisms that are critical to its outcome. These mechanisms enable them to protect themselves in order to survive an asphyctic insult. The most important defense mechanism is the redistribution of the circulation away from the peripheral organs towards critical organs, like the brain. This redistribution is mediated by chemoreceptors and the sympatheticadrenomedullary axis [19-21]. A second mechanism is an actively mediated reduction in energy metabolism and oxygen consumption. Because of these adaptive mechanisms, brain injury can only occur in a narrow window, when the defense mechanisms fall short, causing circulation failure and consequently tissue hypoperfusion [22]. 


\section{Consequences of asphyxia during development}

The impact of asphyxia during development can be somewhat variable. Nowadays, it is accepted that asphyxia might lead to a continuous range of outcomes. Some fetuses do not survive an asphyctic episode, while others survive with short- and/or long-term neurological consequences. Examples are cerebral palsy, mental retardation, epilepsy and learning and memory deficits [16, 23-25]. Some infants will also develop auditory and visual problems [26-30]. Still others survive without any sign of damage.

The pattern of brain damage that will arise after an asphyctic insult depends on the maturational state of the brain as identified by recent magnetic resonance imaging studies. Asphyctic infants born full-term will develop a different injury pattern than asphyctic infants born prematurely [31]. In the full-term asphyctic infant mainly two different patterns have been identified, affecting both neuronal and white matter structures [31-35]. The first pattern involves injury to the watershed regions of the parasagittal cortex and the underlying subcortical white matter, with relative sparing of the thalamus and the basal ganglia. This kind of injury is seen most frequently, occurring in approximately 45\% of asphyctic infants at term. It is usually associated with a less severe, but chronic or repeated asphyxia [36]. The second pattern, occurring in about 25\% of term asphyctic infants, is injury to central nuclei like the basal ganglia, thalamus, cerebellum and brain stem, with relative sparing of the cerebral cortex [33, 37-39]. This type of brain damage often is associated with acute profound, near terminal asphyxia. In addition, clinical evidence is available showing that the hippocampus is vulnerable to asphyxia at term $[33,40]$. Typically, damage to the hippocampus is not observed independently, but combined with damage to the thalamus/basal ganglia and/or a widespread grey and white matter damage. Altogether, asphyxia in near-term infants seems to be relatively selective for brain regions like the thalamus, basal ganglia (mainly putamen) and the cortex. This is probably due to their position within the excitatory neuronal circuits [4, 41]. These regions are all targets of a major glutamatergic input, predisposing them to glutamate-mediated excitotoxicity (Figure 2). 


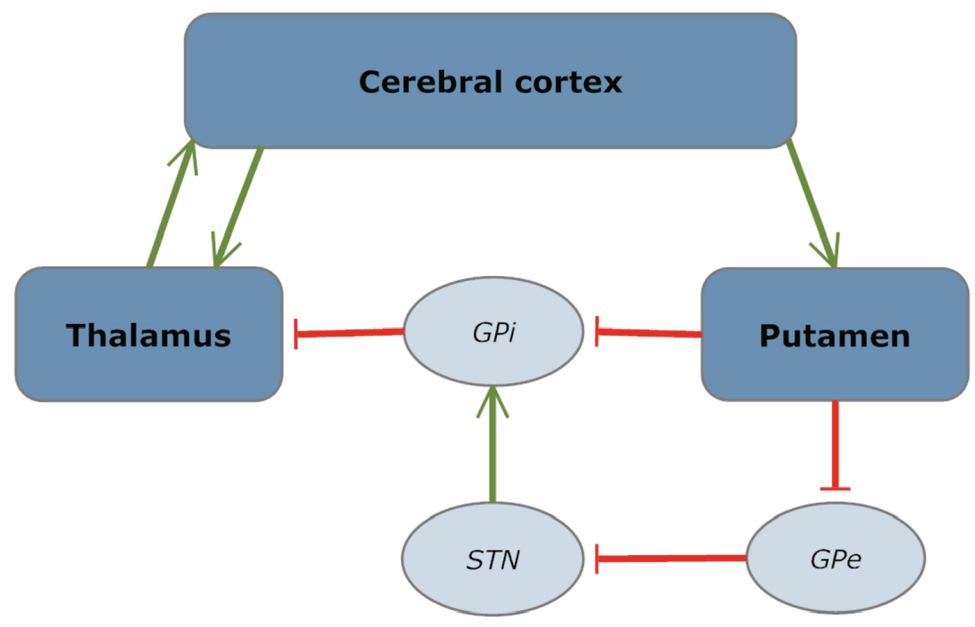

Figure 2: A schematic overview of the regions particularly vulnerable to asphyxia at term, i.e. the cortex, thalamus and putamen (dark blue squares). The green arrows represent excitatory glutamatergic synapses and the red blunted lines represent inhibitory GABA-ergic synapses (Adapted from Johnston et al. [4].

Although the etiology of brain injury in premature infants is complex and multifactorial, periventricular leukomalacia and periventricular/intraventricular haemorrhage are the most frequently diagnosed consequences in those infants [42]. These lesions can either be focal, necrotic lesions in the periventricular white matter or a more diffuse injury of the cerebral white matter. This selectivity for the white matter in the premature newborns may be explained by the characteristics of the oligodendrocytes [43-45]. Preoligodendrocytes and oligodendrocyte progenitor cells seem to be more vulnerable than mature oligodendrocytes, especially for oxidative stress during hypoxia-ischemia. In addition, it is currently thought that white matter lesions are followed by the impaired development of the underlying grey matter regions [46]. Recent MRI studies show reduced volumes of the cerebral cortex, thalamus, basal ganglia and hippocampus of premature infants as well [47-50]. In conclusion, asphyctic encephalopathy reflects a wide range of injury patterns depending on maturational state and severity. 


\section{Current knowledge on experimental models of asphyxia during development: an overview}

In order to better understand the pathophysiological processes leading to neonatal asphyctic encephalopathy after asphyxia, several animal models have been developed, concentrating either on fetal or early postnatal asphyxia [5153]. The choice of animal species or model depends on the research question. Rodents are most frequently used, because they are easily handled, inexpensive and suitable to study both short- and long-term effects. Therefore, a lot of useful data are already available on the physiology, neurochemistry and behavior of both normal and asphyctic animals. Although brain development in sheep and piglet models resembles that of the human infants more closely, these models are very expensive and more difficult to handle [54].

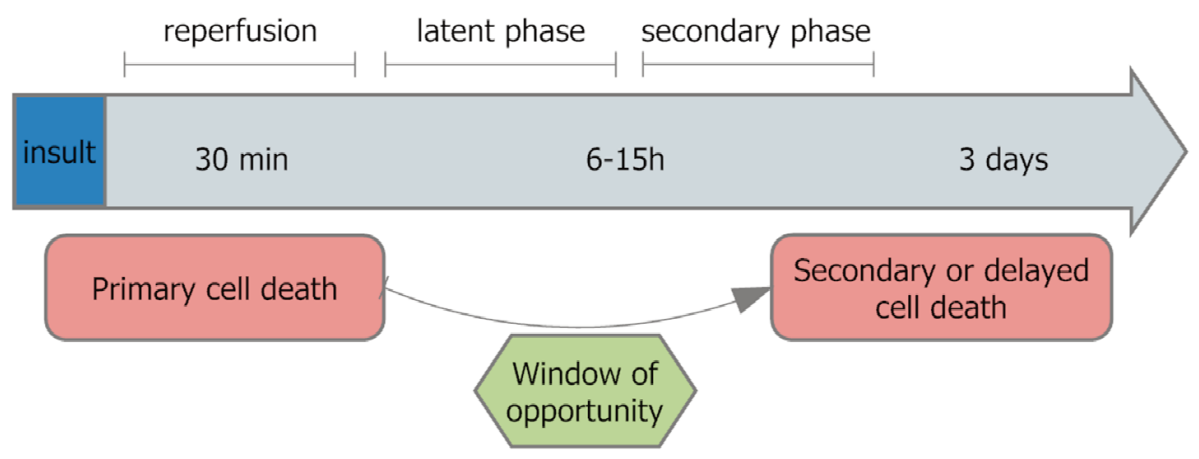

Figure 3: Phases of cerebral injury following a hypoxic-ischemic insult (Adapted from Gunn et al. [2].

The most widely used rat model, the Rice-Vannucci model, is induced by unilateral carotid artery occlusion in a 7-day-old rat pup, followed by a period of systemic hypoxia $[55,56]$. This model produces a stroke-like injury pattern, which is usually restricted to the hemisphere ipsilateral to the occlusion, resulting in damage to the cortex, the subcortical and periventricular white mat- 
ter, the striatum and the hippocampus. A second model rat model, less frequently used, is that of 'delayed Caesarian section' [57]. This model mimics the conditions of asphyxia during human labour. Perinatal asphyxia is induced by removing the uterine horns still containing the fetuses and submersing them in a water bath of $37^{\circ} \mathrm{C}$ for $10-20$ minutes. This insult usually results in primarily apoptotic cell death in different areas of the brain, like the striatum, hippocampus, cortex and cerebellum [58-61]. A last model, that was reported by Cai and colleagues, is an intrauterine hypoxia model, in which the uterine vasculature is clamped for 30 minutes at embryonic day 17 [62, 63]. The models used in this thesis were based on these last two animal models, as will be explained further in the introduction.

Using the models mentioned above, significant advances have been made during the last few decades in understanding the pathophysiological mechanisms leading to post-asphyctic encephalopathy. The major pathogenic mechanisms include programmed cell death, glutamate receptor-mediated excitotoxicity and oxidative stress [64-68]. Injury evolves during one of the four phases of the hypoxic-ischemic insult (Figure 3) [2].

During the primary phase, i.e. the actual hypoxic-ischemic event, cerebral energy failure develops, resulting in an overstimulation of the neuronal glutamate receptors and a primary wave of cell death. Calcium-dependent glutamatergic excitotoxicity is one of the main mechanisms suggested to be involved [69]. In this cascade, the depletion of oxygen and glucose leads to deprivation of the energy stores in the cell, which in turn leads to malfunctioning of the $\mathrm{NA}^{+} / \mathrm{K}^{+}$ATPases through lack of adenosine triphosphate (ATP) and depolarization of the cell [70-72]. The absence of a proper membrane gradient causes an increased release as well as an impaired reuptake of glutamate, mediating a toxic buildup of extracellular glutamate in the synaptic cleft $[73,74]$. This leads to an overstimulation of glutamate receptors, like the N-methyl-Daspartic acid (NMDA) and alfa-amino-3-hydroxyl-5-methyl-4-isoxazoleproprionate (AMPA) receptors in the postsynaptic membrane, inducing a lethal calcium influx in the cell. In the cytosol, excessive calcium amounts trigger the activation of proteases, lipases and endonucleases, which subsequently lead to cell damage and often cell death [75]. Moreover, the increased activation of NMDA receptors can also lead to the production of oxygen free radicals 
through the activation of cyclooxygenase and lipooxygenase pathways, the activation of nitic oxide synthase (NOS) and the activation of proteases and phospholipase C, which can all contribute to cell death (reviewed by [76]). An overview of this cascade is given in figure 4.

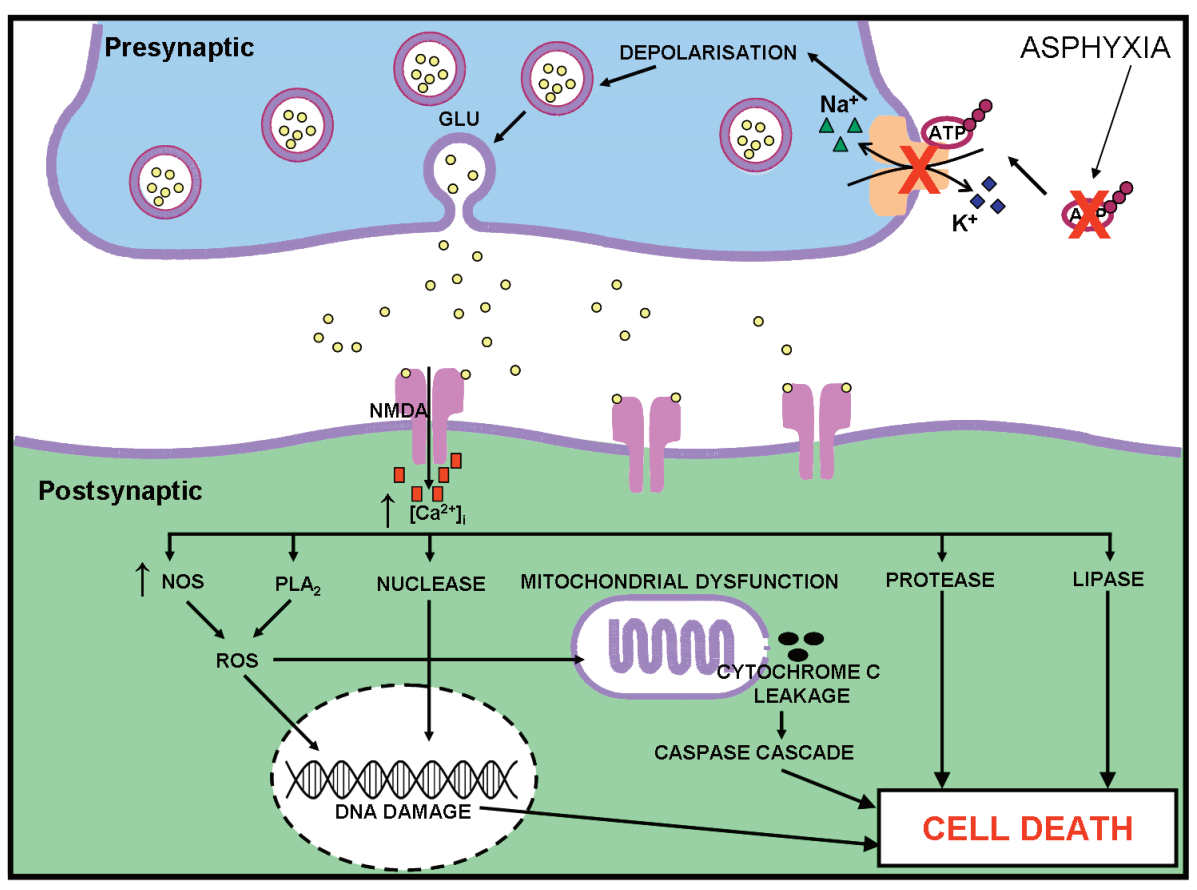

Figure 4: An overview of the mechanisms causing cell death after asphyxia

As reperfusion occurs, characterized by the partial recovery of cellular oxidative and metabolic processes, injury further evolves [2]. Oxygen and glucose substrates become available again, leading to the formation of more oxygen free radicals, like superoxide anion, hydrogen peroxidase and hydroxyl radicals [7779]. Free radicals further initiate injury through lipid and protein oxidation as well as DNA base modifications [80]. The immature brain is more vulnerable to oxidative stress than the adult brain, because of its high concentrations unsaturated fatty acids, high rate of oxygen consumption, low concentrations of anti-oxidants and increased availability free redox-active iron [66, 81-85]. In addition, radicals also trigger mitochondrial dysfunction and leakage of mito- 
chondrial components, like cytochrome $\mathrm{C}$ that can activate the apoptotic cascade.

The reperfusion phase is followed by the latent phase, in which there is a complete normalization of the oxidative energy metabolism [2].

In the end, the latent phase is followed by a secondary deterioration phase, usually starting as late as 6-15 hours after the initial insult [2]. In this phase, cerebral blood flow is markedly increased and after a while a secondary fall in cerebral blood flow occurs. These events ultimately lead to secondary or delayed cell death, during which neurons and/or oligodendrocytes continue to die for longer periods [61]. Little is known about the exact mechanisms leading to this delayed phase of cell death. It is speculated that it is the result of a complex physiological cascade, comprising distinct events. Because dying cells are only detectable for a restricted time window of 8-11 hours before being removed by microglia, delayed apoptosis cannot be the direct result of the insult or reperfusion itself. Apoptotic cells are visible until postnatal day 15, peaking at postnatal day 7-8 [58]. The critical processes, which start as early as 6 hours after the insult, probably involve the accumulation of toxic metabolites like glutamate, the overproduction of free radicals, the release of cytochrome C into the cytosol and caspase- 8 activation and DNA cleavage by caspase 3 $[61,86]$. In addition, it is currently thought that delayed cell death may be apoptotic and/or necrotic and that both forms of cell death may not be mutually exclusive. Hybrid cells can be seen that display morphological features of both types. This has been described as the 'apoptosis-necrosis continuum' [61, 87]

It is important to note that there is an time window of opportunity for intervention, since there is a time frame of 3-8 days after the insult prior to the final execution phase of delayed apoptotic cell death [58]. This window of opportunity is also depicted in figure 3.

In contrast to the morphological and biochemical changes described above, less is known about the behavioral consequences of asphyxia during development. Locomotor and sensimotor behavior are mostly studied in young and young adult animals. Open field testing revealed mainly hyperactivity at weaning and early postnatal ages in the Rice-Vannucci model, the delayed Caesarian section model as well as in the intrauterine hypoxia model [88-93]. At 6 
weeks of age, the Rice-Vannucci model also demonstrated other motor deficits in the rotarod test, the grip task and limb placing and foot-faults [94-96]. Only one study showed such sensimotor problems after global asphyxia [97]. Learning and memory impairments were found as well in all different animal models. The Rice-Vannucci model resulted in impaired learning and memory performance in the Morris Water Maze, T-maze acquisition, the choice reaction time task and plus-maze tasks at 6-16 weeks of age [89, 98-100]. Intrauterine hypoxia also impaired learning abilities in 1-month-old rats in the Morris water maze and passive avoidance test [93]. Similarly, global perinatal asphyxia impaired memory performance starting from 4 months of age [101]. Perinatal asphyxia caused spatial and non-spatial learning problems in the Morris Water Maze and object recognition task at 4, 18 and 24 months of age [57, $97,101,102]$. In these aged animals, anxiety-related behavior and social interaction were also affected, as seen by the decreased social aggressiveness and increased social contact and anxiety levels [103].

\section{Strategies for neuroprotection: hypoxic-ischemic preconditioning}

Nowadays, the treatment of neonates at risk for asphyctic encephalopathy is mainly supportive, involving blood pressure control, seizure control, assisted ventilation and medications to support the infant's breathing. There are almost no clinically approved treatments available that improve their outcome or prevent damage. However, many different treatment options are currently under investigation. These include methods to inhibit glutamate release, block the NMDA receptors, reduce oxygen free radicals, modulate inflammatory processes or inhibit apoptosis [64, 104-106]. Although many of these interventions seem to ameliorate brain injury in animal models, most clinical trials have not been that successful. Only mild post-asphyctic hypothermia improves the outcome of some asphyctic near-term infants. For example, Gluckman et al. reported that head cooling was beneficial in near-term infants with moderate post-asphyctic encephalopathy compared to conventional care [107-109]. In addition, Shankaran et al. demonstrated a decrease in percentage of death or 
moderate-to-severe disabilities from 62\% in the control group to only $44 \%$ in the group with whole-body mild hypothermia [ $110-114]$. The mechanism of action is, however, still unclear. Although this strategy has become the standard care in near-term asphyxia, further research is still necessary to find the ideal drugs and therapies to prevent post-asphyctic encephalopathy more efficiently.

An interesting approach to further study neuroprotection is investigating the concept of preconditioning. Practically any stimulus capable of causing injury to a tissue or organ can, when applied close to the threshold of damage, activates endogenous protective mechanisms $[1,5]$. Thus, it can lessen the impact of a subsequent more severe stimulus. Ischemic preconditioning was first reported by Murray and colleagues in 1986 [115]. They showed that a series of short coronary artery occlusions followed by reperfusion can protect the heart against a subsequent lethal ischemia. This protection against a potentially recurring challenge can also be applied to asphyxia in the brain and then it is called 'hypoxic-ischemic/asphyctic preconditioning' or 'tolerance'. The basis principle is shown in figure 5.

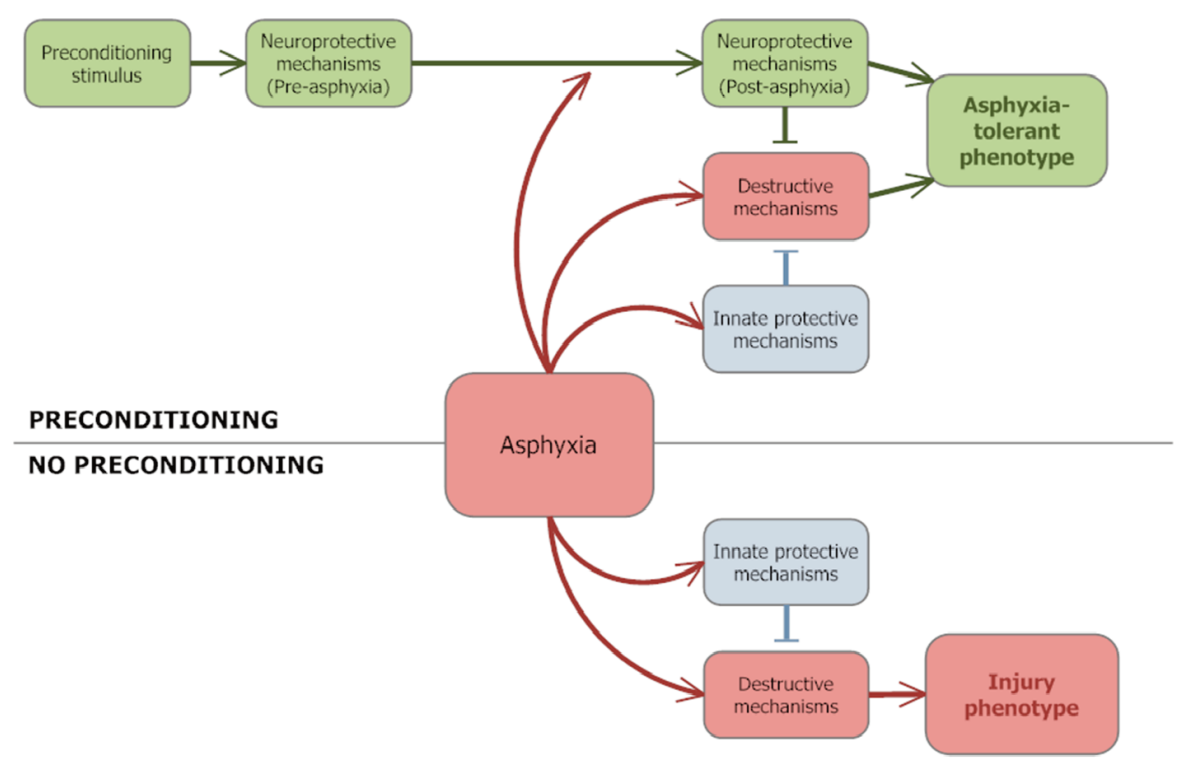

Figure 5: A schematic overview of neuroprotection by preconditioning (Adapted from Gidday et al. [1] and Dirnagl [5]. 
There are two temporally and mechanistically distinguishable forms of preconditioning observed in the brain: a short-lasting or acute form and a longlasting or delayed form $[1,5]$. The acute form is mediated by post-translational modifications of proteins like ion channels and is short-lived, while the delayed form is mediated by the new protein synthesis and sustains for days or even weeks [1 16-1 18]. Understanding these protective signaling pathways and the identification of drugs that can mimic these protective responses are important steps towards improving the outcome of infants at risk for asphyctic encephalopathy.

\section{Current knowledge on experimental models hypoxic-ischemic preconditioning in the immature brain: an overview}

Nowadays, many experimental models are being used, involving different preconditioning stimuli, like brief ischemia or hypoxia, oxidative stress, lipopolysaccharides, anesthetics in either adult or immature rats, mice and gerbils [1, 119 -123]. These preconditioning models can be differentiated into neonatal or early postnatal and prenatal models.

\section{Neonatal and early postnatal models}

The most often used model, i.e. postnatal hypoxic preconditioning, was introduced by Gidday and colleagues in 1994 [124]. Exposure of a 6-day-old rat pup to hypoxia $\left(8 \% \mathrm{O}_{2}\right.$ for $\left.3 \mathrm{~h}\right)$, used as a preconditioning stimulus, was followed by the Rice-Vannucci model one day later. Gidday and colleagues have shown that this type of preconditioning can reduce the infarct volume in the immature brain. Other studies using similar animal models confirmed these results $[55,125]$. A lower incidence in brain damage, a reduction in lesion volume and a decrease in the number of apoptotic cells in areas like the cortex, hippocampus and striatum were the main findings [55, 125-130]. 
The exact mechanisms underlying this hypoxic preconditioning phenomenon still remain unclear. Nevertheless, a few important finding have already been made in this model. A first finding is that nitric oxide (NO) and NOS seem to play a critical role. Gidday et al. have reported that especially endothelial NOS (eNOS) is required. Furthermore, hypoxia-inducible factor-1 (HIF-1) seems to be involved in the establishment of a neuroprotective state [131]. Jones et al. and Bernaudin et al. both showed an increased HIF-1 expression [132, 133]. In addition, the expression of different HIF-1 target genes important for cell survival, glycolysis and angiogenesis, like GLUT-1, erythropoietin (EPO), lactate dehydrogenase and vascular endothelial growth factor (VEGF), was upregulated as well in preconditioned animals $[119,132,134]$. Another important finding is that a hypoxic preconditioning stimulus influences the energy metabolism. Brucklancher et al. showed that preconditioned animals show an increase in brain glycogen and ATP compared to non-preconditioned animals [135]. Accordingly, Vannucci et al. also reported a more optimal preservation of highenergy reserves, like ATP and phosphocreatine. Two additional important findings are that rapid, intermediate and long-term neuroprotection in the neonatal brain all activate CAMP response element binding (CREB) [129, 136] and that preconditioning induces the expression of heat-shock protein (HSP) 72 in different areas of the brain, especially in endothelial cells [122, 137]. CREB is an important transcription factor in neuronal development and plasticity, while HSP 72 is a stress protein that plays an important role in the stabilization of existing and correct folding of new proteins [138, 139].

\section{Prenatal models}

Besides early postnatal or neonatal animal models, also a few prenatal preconditioning models have been reported. Cai and colleagues clamped the uterine vasculature for 30 minutes as a preconditioning stimulus, followed by ligation of the right common artery at P7 [93]. Using this model, they found that prenatal hypoxic-ischemic preconditioning reduced the infarct volumes and neuronal damage, probably mediated by HSP70. In the same model, Xiao et al demonstrated that a reduction in caspase-3 activity and NO seems to contribute to this type of tolerance [121]. Zhao et al induced tolerance by exposing the pregnant dam to 30 minutes of $15 \%$ hypoxia. They have shown that 
neuroprotection is inducible NOS (iNOS)-dependent [140].

\section{Current limitations: Why novel animal models?}

One of the important limitations of the current asphyxia and hypoxic-ischemic preconditioning studies is that almost all animal models use a focal insult, like unilateral carotid artery occlusion, in postnatal rat pups [55, 124]. These models merely cause one-sided focal brain damage with the formation of cavitary lesions, which does not mimic the most common pathological type of global asphyctic encephalopathy and multiple organ failure in the human neonate. Furthermore, those current models do not take into account the unique physiological mechanisms specific to the time of birth that may also play an important role in the development of asphyctic brain injury. For these reasons, two new rat models were used in thesis (Figure 6). The first model used studies severe fetal asphyxia. The uterine vasculature is completely clamped for 75 minutes at embryonic day 17 (E17). The second model used, induces mild fetal asphyxia as preconditioning stimulus. At E17, the uterine vasculature is clamped for 30 minutes, according to Cai et al. in 1997 [121, 130]. In contrast to their model, the stimulus was followed by a perinatal insult at E21/P0 to induce severe asphyxia. A Caesarian section was performed to remove both uterine horns still containing the pups and these horns were submersed in a water bath $\left(37^{\circ} \mathrm{C}\right)$ for 19 minutes.

Both animal models used in this thesis are, therefore, global events that are associated with both systemic metabolic and hemodynamic changes that might also influence the outcome of the fetus. Therefore, these models, with the described consequences, mimic the global, pathophysiological conditions of asphyxia before and during birth. Furthermore, the unique physiological mechanisms specific to the time of birth, involving hormonal changes, might play an important role in the development of asphyctic encephalopathy during the parturition process itself. In addition, the degree of energy consumption between a near-term fetus and neonatal animal is quite different. Overall, the fetus is more resistant to hypoxia than the neonate [141-143]. For these reasons, it is particularly important to study the multifactorial effects of asphyxia and preconditioning during the processes of pregnancy and delivery. The in- 
sults used in this study, therefore, reflect the clinical situation more closely than stroke, which is only rarely seen in human newborns (approximately 1 in 4000 term births) [144].

However, one could argue that the rat brain at birth is more immature than that of the human newborn at term. With regard to synaptogenesis and myelination, the rat brain resembles the human equivalent at approximately 10 days after birth [145-148]. Nonetheless, premature neonates more often suffer from perinatal asphyxia and hypoxic-ischemic encephalopathy than term infants, making this maturational status clinically relevant as well [149-151].

A second important limitation of the current asphyxia and preconditioning studies is that almost all reports focus on the immediate, histological outcome [124-126]. Developmental encephalopathy and neuroprotection are typically established by quantifying the infarct volume or the degree of cell death a few days after the insult, i.e. 1 week or shorter. Nevertheless, hypoxic-ischemic injury is a dynamic process extending up to weeks or even months after the initial injury [58, 152-154]. Furthermore, hypoxia-ischemia does not necessarily result in both explicit brain damage on a morphological level and observable deficits in brain functioning or behavior $[89,155]$. Histological preservation does not necessarily mean that neuronal functioning and behavior is preserved and visa versa, which is impossible to establish in short-term histological studies. In addition, some of the protective strategies might just delay the injury rather than providing a lasting protection $[156,157]$. These might be reasons why some clinical neuroprotective strategies failed, despite good protective results in short-term, morphological animal studies [158]. Consequently, it is important to study the long-term, functional consequences as well Therefore, the studies in this thesis mainly concentrated on the long-lasting, morphological consequences combined with functional/behavioral analyses of both fetal asphyxia and hypoxic-ischemic preconditioning.

\section{Aim and outline of the thesis}

As mentioned in paragraph 5, two different animal models were used in this thesis: a model for severe fetal asphyxia and a model for fetal asphyctic pre- 
conditioning. An overview of the different animal models and experiments is given in figure 6 .

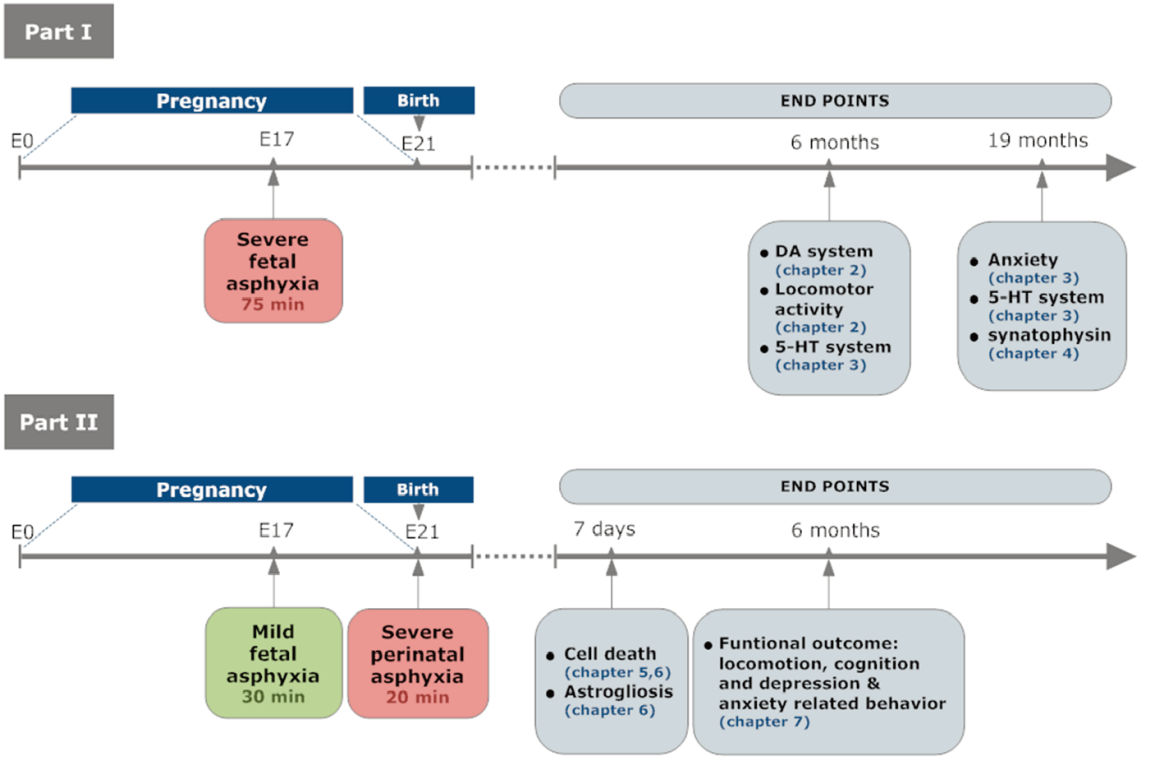

Figure 6: An overview of the different models and experiments in this thesis.

The first part of this thesis aimed at exploring the long-term consequences of severe fetal asphyxia both at a behavioral and morphological level. Fetal asphyxia was induced at E17 by completely clamping the ovarian and uterine arteries for 75 minutes, followed by reperfusion. In chapter $\mathbf{2}$, we present the long-term effects of fetal asphyxia on spontaneous locomotion and the dopaminergic nigrostriatal system. In this study, 6-month-old animals were tested in the open field task. Afterwards, the number of dopaminergic neurons was counted in the substantia nigra pars compacta. In Chapter 3, we investigated the long-term consequences of fetal asphyxia on anxiety-related behavior and on the serotonergic dorsal raphe neurons in the aging rat. Chapter 4 highlights the long-lasting effects of fetal asphyxia on striatal and prefrontal synapses. The number and density of presynaptic boutons were estimated in 19-month-old animals using a synaptophysin antibody. 
In contrast to the first part, the second part of this thesis discusses the consequences of mild fetal asphyxia as a preconditioning stimulus. This second part aimed at developing and validating a new rat model for hypoxic-ischemic preconditioning, because of the limitations mentioned in paragraph 5. Our new model combines mild fetal asphyxia, i.e. 30 minutes as described above, with severe perinatal asphyxia at birth. To induce severe perinatal asphyxia, a Caesarian section was performed by remove both uterine horns still containing the pups and these horns were submersed into a water bath of $37^{\circ} \mathrm{C}$ for 19 minutes. We wanted to validate our model for short-term, morphological neuroprotection as well as long-term functional protection. Chapters 5 and 6 concentrate on this first aspect by analyzing the degree of apoptotic cell death in different brain areas of the 8-day-old preconditioned rat pup. Chapter 7 addresses the long-term functional consequences. Spontaneous locomotor activity, anxiety and depression-related behavior and learning/memory were assessed in 6-monh-old preconditioned rats. Finally, in chapter 8, an overall conclusion of our experimental findings and possible future implications is provided. 

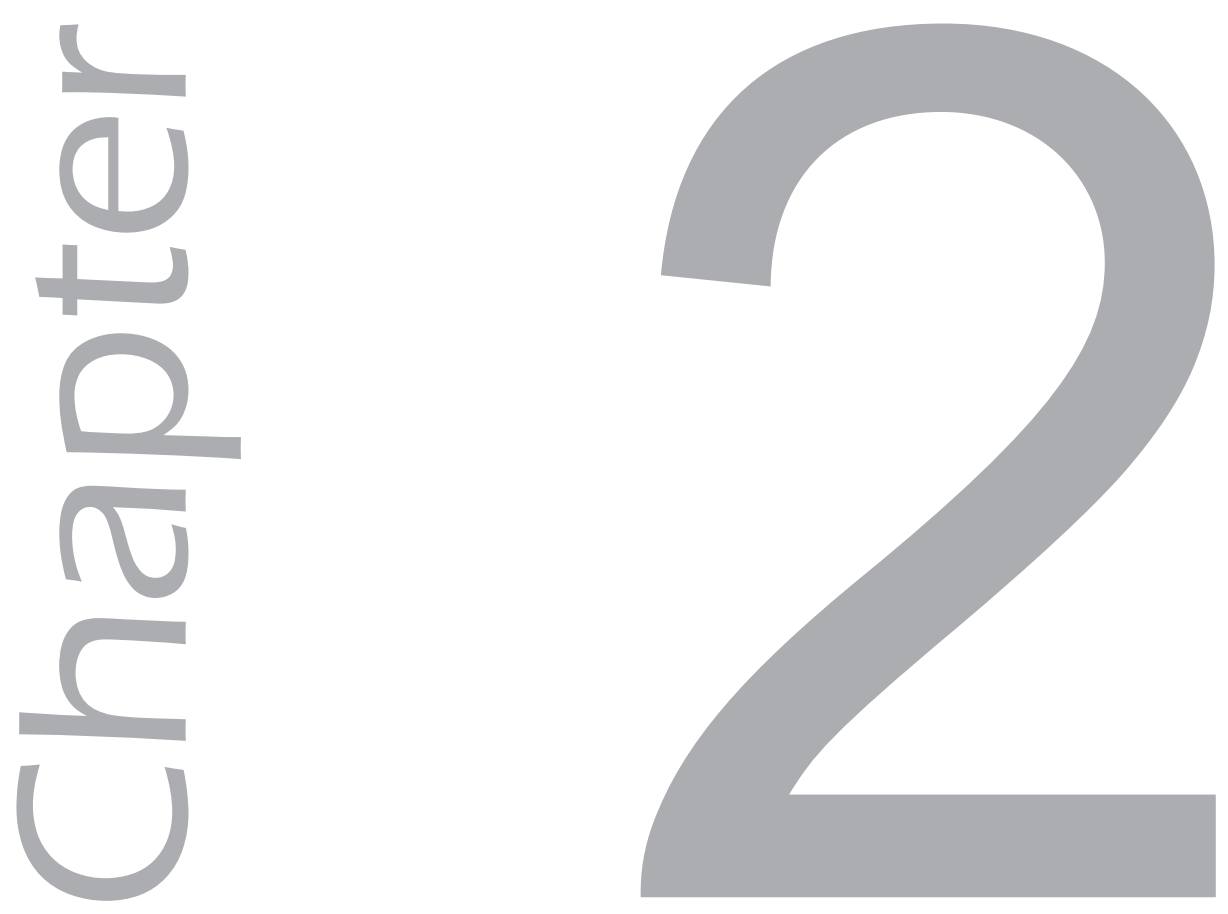

A combined behavioral and morphological study on the effects of fetal asphyxia on the nigrostriatal dopaminergic system in adult rats

E. Strackx, D.L.A. Van den Hove, H.P. Steinbusch, H.W.M. Steinbusch, J.S.H. Vles, C.E. Blanco and A.W.D. Gavilanes 


\section{Abstract}

Fetal asphyxic insults in the brain are known to be associated with developmental neurological problems like neuromotor disorders. However, little is known about the long-term consequences of fetal asphyxia (FA). For that reason, the present study investigated the long-term effects of FA on motor behavior and dopaminergic circuitry. FA was induced at embryonic day 17 by 75-minute clamping of the uterine and ovarian arteries. SHAM animals underwent the same procedure except for the clamping. This was followed by fullterm vaginal delivery of animals in all groups (FA, SHAM and untreated controls). At 6 months, basal and amphetamine-induced locomotor activity were measured during open field testing. Brain sections were stained for tyrosine hydroxylase (TH) and glial fibrillary acidic protein (GFAP). TH-positive cells and GFAP-positive cells in substantia nigra pars compacta (SNC) and striatum were counted using design-based stereology. Moreover, TH-immunoreactivity in the striatum was assessed by grey value measurements. Behavioral analysis demonstrated that SHAM and FA showed less basal and amphetamine-induced activity than controls. Histochemically, FA decreased the number of TH-positive neurons in the SNC and lowered TH-positive in the striatum. Further, more GFAP-positive cells were found in the SNC and striatum in FA than in either control and SHAM groups. The data show that FA was associated with deficits in both dopamine-related motor behavior and biochemistry. These alterations were associated with nigrostriatal astrogliosis. The present study demonstrates the sensitivity of the dopaminergic system towards FA. 
sphyxic insults in the developing brain, whether pre-, peri- or post-
natal, are the leading cause of neonatal mortality and morbidity
and may have dramatic neurological consequences, including
motor impairments like cerebral palsy in those children that survive [159-161]. Additionally, asphyxia is presumed to contribute to the development of psychiatric disorders like schizophrenia and autism as well as neurodegenerative disorders in adult life [162-166].

Several experimental animal models have been developed to study the effects of asphyxia on brain development. Most of them are limited to (full-term) neonatal models, investigating the neurological consequences of either perinatal or early postnatal asphyxia [reviewed in [52, 167]]. The model most widely used is the Rice-Vannucci model, combining unilateral carotid artery ligation with exposure to hypoxia for several hours in 7-day-old rat pups (P7) [168]. Another frequently used model is a delayed caesarian section, inducing severe perinatal asphyxia (SPA) at P0 [166]. However, potential complications in the developing brain during or shortly after delivery can only explain a small part of the impairments seen in children's behavior and development [169]. Numerous epidemiological studies have demonstrated that prenatal factors, with fetal inflammation and hypoxia-ischemia being the most important ones, are essential predictors of postnatal neurodevelopmental outcome [170-172]. Nevertheless, little attention is paid to models investigating the consequences of asphyxic insults during this prenatal period. For that reason, this study used a fetal asphyxia model, which reflects the pathological conditions of global asphyxia induced in a very early stage of brain development.

Currently, there is growing evidence, in both humans and animal models, that dopamine and the dopaminergic neurocircuitries are extremely sensitive to hypoxia and/or ischemia around birth [173-177]. SPA studies (19-20 minutes) show both short- and long-term deficits, among which are less dopaminergic neurons, a decreased level of dopaminergic receptor D1 and lowered extracellular dopamine levels together with a decreased presynaptic dopamine release in both the substantia nigra (SN) and striatum [178-180]. Using the Rice-Vannucci model, a similar loss in dopaminergic neurons was found [181, 182]. Both the SN and striatum are part of the mesostriatal dopaminergic pathway, which is one of the systems involved in the control of motor behavior 
[183]. Therefore dysregulation of dopamine function in the basal ganglia might be responsible for motor disturbances. Interestingly, 15-16 minutes of SPA induced hyperactivity, while 19-20 minutes of SPA resulted in hypoactivity during open field testing $[88,166]$. The effects of asphyxia during the fetal period on these dopamine-mediated systems are unknown. However, the fetal period is very important for the ontogeny of dopaminergic structures, like the SN. $\mathrm{BrdU}$ and $[3 \mathrm{H}]$ thymidine experiments have shown that cell division of nigral precursor cells in the subventricular zone (SVZ) occurs between E12 and E15 [184-187]. Between E15 and E18 these nigral precursor cells migrate from the SVZ to the SN $[188,189]$. By E20 the dopaminergic neurons have topography similar to the adult brain. In addition, the first dopaminergic projections reach the striatum by E14, but most of the innervation takes place between E18 and P3 [190]. For that reason, it seems plausible that an insult during this fetal period can cause profound effects on the embryonic development of the nigrostriatal neurons and projections.

The aim of the present study was to determine long-term effects of fetal asphyxia (FA) on motor behavior and dopaminergic circuitry at the age of 6 months. For this purpose, the uterine circulation was clamped for 75 minutes at embryonic day 17 (E 17). Basal and amphetamine (AMPH)-stimulated motor behavior were tested in an open field. Additionally, brains were analyzed for tyrosine hydroxylase (TH) and glial fibrillary acidic protein (GFAP) immunoreactivity in the substantia nigra pars compacta (SNC) and striatum. This is the first report demonstrating long-term changes in dopamine-related locomotion and biochemistry due to a fetal asphyxic insult.

\section{Materials and methods}

\section{Animals}

All experiments were approved by Animal Ethics Board of Maastricht University on animal welfare according to Dutch governmental regulations. All efforts were made to minimize the pain and stress levels experienced by the animals and to minimize the number of rats necessary to produce reliable sci- 
entific data. Inbred date-pregnant Lewis rats were supplied on day 14 of gestation by the Animal Care Department of Maastricht University and were housed individually at its facilities. The rats were kept under standard laboratory conditions with food and water given ad libitum, $21 \pm 2^{\circ} \mathrm{C}$ environment temperature, a 12h light/dark schedule (lights on at 07:00 h) and background noise provided by radio.

\section{Fetal asphyxia procedure}

At E17, pregnant rats were anaesthetized by xylazine (1 mg/kg s.c.) and ketamine $(50 \mathrm{mg} / \mathrm{kg}$ i.m.). Rats were subjected to a midline laparotomy to expose both uterine horns. All procedures were performed within a controlled environment $\left(37^{\circ} \mathrm{C}, 80 \%\right.$ humidity, room air). Fetal asphyxia was achieved by completely clamping both uterine and ovarian arteries with removable clamps. In the mean time, the uterine horns were kept wet, by rinsing them with saline $0.9 \%$. Additionally, dams were injected subcutaneous with saline $0.9 \%$ $(5 \mathrm{ml} / 100 \mathrm{~g}$ interscapularly) to prevent dehydration. After 75 minutes, the clamps were removed to allow reperfusion. The uterine horns were placed back intra-abdominally and the abdominal wall was repaired. Sham-operated rats underwent the same procedure except for clamping the arteries. The fetal asphyxic (FA, $n=10$ ), sham-operated (SHAM, $n=10$ ) and control (C, $n=10$ ) groups were allowed to deliver normally (i.e. full-term vaginally delivered on E21-22). Exclusively male offspring were used within this study, because both morphological and behavioral evidence show a differential vulnerability to a birth insult in males versus females. A greater impact is seen in the male gender, probably due to the protecting role of the circulating estrogens in females [191, 192]. No more than two pups per litter were used to prevent litter effects. They were randomly cross-fostered to surrogate dams, which have given birth normally the same day.

\section{Behavioral testing}

Open field motor activity was assessed at the age of 6 months under saline $0.9 \%$ and D-amphetamine (AMPH)-conditions $(0.5$ and $1.0 \mathrm{mg} / \mathrm{kg}$; intraperitoneally). Locomotor activity was evaluated by placing the rat in the centre of 
a 50 by $50-\mathrm{cm}$ square with a $30-\mathrm{cm}$ high enclosure. Their locomotor behavior was recorded for 60 minutes with a video-camera attached to a computer running the Ethovision ${ }^{\mathrm{TM}}$ programme (Noldus Equipment, Wageningen, The Netherlands). All animals were subjected to one trial a day for 3 consecutive days. Distance moved and mean velocity while moving per 10-minute interval were scored. Of note, background noise was provided by radio during the tests.

\section{Perfusion and tissue preparation}

Following completion of the behavioral experiments, the animals were deeply anaesthetized with sodium pentobarbital $(60 \mathrm{mg} / \mathrm{kg}$ i.p.) and transcardially perfused with ice-cold $4 \%$ paraformaldehyde in $0.1 \mathrm{M}$ phosphate buffer $(\mathrm{pH} 7.6)$. The brains were dissected rapidly from the skull and post-fixed for $2 \mathrm{~h}$ at $4^{\circ} \mathrm{C}$ in the same fixative. The brains were suspended in $15 \%$ sucrose. Brains were cut serially into 30 micrometer thick coronal cryostate sections at the following bregmas: for striatum and lateral ventricles bregmas $1.60 \mathrm{~mm}$ to $-0.80 \mathrm{~mm}$ and for SNC bregma $-5.20 \mathrm{~mm}$ to $-6.30 \mathrm{~mm}$.

\section{Immunohistochemistry}

To evaluate the degree of neuronal dopaminergic changes in the SNC and striatum, sections were immunostained with mouse anti-tyrosine hydroxylase (TH) antibodies (TOHA 1.1 kindly supplied by Dr. C. Cuello, Canada) at a dilution of 1:100, followed by incubation with donkey anti-mouse biotinylated secondary antibodies (1:400; Jackson Immunoresearch Laboratories, West Grove, USA). Astrocytic changes were visualized using glial fibrillary acidic protein (GFAP) as a marker. Cryostat sections were labeled with mouse anti-GFAP (1:100; Innogenetics), using donkey anti-mouse biotinylated immunoglobulins as secondary antibodies (1:200; Jackson). Brain sections were further processed for immunohistochemistry using the avidin biotin complex technique with 3,3diaminobenzidine (DAB) to obtain a color reaction (Vector laboratories, USA). All immunohistochemical reactions were carried out in a free-floating manner. After the labeling procedures, sections were mounted on gelatine-coated glass slides, air-dried, dehydrated in ascending ethanol concentrations and cover- 
slipped with depex. Sections from FA (n=6), SHAM (n=6) and control (n=4) animals were processed simultaneously under the same experimental conditions.

\section{Design-based stereology}

Design-based stereology was performed for volume measurements and cell counts, using a stereological computer microscopy system and Stereolnvestigator software (MicroBrightField, Williston, VT, USA). The regions of interest (e.g. total brain, ventricles, striatum, SNC and cortical grey matter) were delineated according to Paxinos and Watson (1986) by anatomical landmarks with a 1.25x magnification on live microscopic video images displayed on a monitor. Volumes were estimated by multiplying the average surface area with the section thickness, the number of sections investigated, and a factor of eight since every eighth section was used (section sampling fraction (ssf) $=1 / 8$ ). White matter volumes were measured, using the Cavalieri's point counting method, as follows. A counting grid (300 x $300 \mu \mathrm{m}$ ) was randomly placed over the sections and the points hitting the white matter were counted. The white matter volume was then calculated by multiplying the average sections thickness, the grid size, the ssf and the total number of points counted [193].

The TH and GFAP-positive cells were counted with the optical fractionator method [193]. Briefly, counting frames were created by the software and placed at the intersections of a grid that had been randomly placed over the section. The counting frames were replaced systematically by stepwise movements in $x$-and $y$-directions. The area of the counting frame relative to the area associated with the $x$ and $y$ steps gives the area sampling fraction (asf). The optical dissector height, along the z-axis, was determined by excluding the parts close to the slide and the coverslip. The height of the optical dissector relative to the actual thickness of the section results in the height sampling fraction (hsf). Only counting frames for which at least a part of the frame fell within the delineated contour were used for counting. Cells were marked if they werepositive and in focus within the counting area. Furthermore, the counting frames have forbidden lines to avoid edge effects. The estimated total number of positive cells $(N)$ was calculated from the number of cells counted (Q) according to the formula: $N=1 / s^{*}$ * $1 /$ asf * $1 /$ hsf * $Q$. Details on the stereological counting procedures are summarized in table 1. 
Table 1: Details of the stereological procedure

\begin{tabular}{|c|c|c|c|c|c|c|c|c|}
\hline & obj & B & $\mathrm{H}$ & $D_{x}$ & $D_{y}$ & $\mathrm{t}$ & $\Sigma Q^{-}$ & CEpred $_{[n]}$ \\
\hline TH-SNC & $100 x$ & 440 & 5 & 25 & 25 & 8.7 & 623 & 0.040064 \\
\hline GFAP-SNC & $50 x$ & 4000 & 5 & 90 & 65 & 10.7 & 321 & 0.055815 \\
\hline $\begin{array}{l}\text { GFAP-striatum } \\
\text { obj, Objective used for co }\end{array}$ & $\frac{50 x}{i n g ; B, B a}$ & $\frac{6000}{\text { e area of th }}$ & $\frac{5}{\text { nbias }}$ & $\frac{400}{\text { irtual cou }}$ & $\begin{array}{l}400 \\
\text { ing space }\end{array}$ & $\frac{8.3}{\text { in } \mu m^{2} ; H}$ & $\frac{972}{\text { leight of }}$ & $\frac{0.032075}{\text { iiased virtual count- }}$ \\
\hline $\begin{array}{l}\text { ing spaces in } \mu m^{2} ; \text {, dist } \\
\text { thickness measured after }\end{array}$ & e between & the unbias & & unting $s p$ & es in direc & & & $\begin{array}{l}\text { tual average section } \\
\text { rage predicted coef- }\end{array}$ \\
\hline
\end{tabular}

\section{Grey value measurements}

TH-immunoreactivity in the striatum was measured by a semi-quantitative method. Briefly, photographs were taken from 4 different regions (ventral, medial, dorsal and lateral) within each section with the Olympus AX-70 microscope connected to a digital camera (F-view; Olympus, Tokyo, Japan). To correct for potential variability in lighting conditions, all images were collected under identical conditions. Photographs were analyzed, using their corresponding average grey values (ranging from 0-black to 255-white), obtained with the NIH ImageJ software (http://rsb.info.nih.gov/ij/). Using a trial and error method, a threshold value was selected and kept the same for all measurements. Blood vessels, tissue out of focus or artifacts were excluded from the analyses. Background immunoreactivity was normalized within each sections by dividing the average gray value by the average value of white matter, which has negligible TH-staining.

\section{Statistical analysis}

All data are represented as mean + standard error of means (+ SEM). Stereological and immunoreactivity data were analyzed using a one-way analysis of variance (ANOVA) test, followed by post-hoc comparisons using Bonferroni ttest. For both parameters (distance moved and velocity) of the open field task, the effects of dose and group were analyzed by multivariate ANOVA (MANOVA). Significant effects were further analyzed by post-hoc Duncan corrections. The accepted level of statistical significance was $p<0.05$ for all analyses. All calculations were done using the Statistical Package for the Social Sciences (SPSS 12.0 software). 


\section{Results}

\section{Open Field}

The results of the open field task are depicted in figure 1. Comparison of motor activity in control animals after saline or AMPH injection indicates that both the distance traveled (Fig 1A) and velocity (Fig 1B) were stimulated by AMPH in a dose-dependent manner. There was a significant effect of dose on distance moved (MANOVA; $p=0.0001$; figure 1A). Under basal saline conditions, both SHAM and FA animals showed a significantly reduced distance moved compared with control animals (MANOVA; $p=0.0025$ ). Post-hoc testing revealed no difference between the SHAM and FA group. After $0.5 \mathrm{mg} / \mathrm{kg}$ AMPH injection, no significant differences were found between the different groups. However, after $1.0 \mathrm{mg} / \mathrm{kg}$ AMPH stimulation, both SHAM and FA animals were less active than control animals as indicated by the shorter distances traveled (MANOVA; $p=0.049$ ). Again post-hoc comparisons revealed no differences between the SHAM and FA group. In addition, there was a significant effect of dose on the mean velocity (MANOVA; $p=0.0001$; figure 1B). However, no effect of either SHAM or FA was found on the mean velocity under basal conditions, after $0.5 \mathrm{mg} / \mathrm{kg}$ or after $1.0 \mathrm{mg} / \mathrm{kg} \mathrm{AMPH}$-stimulation.
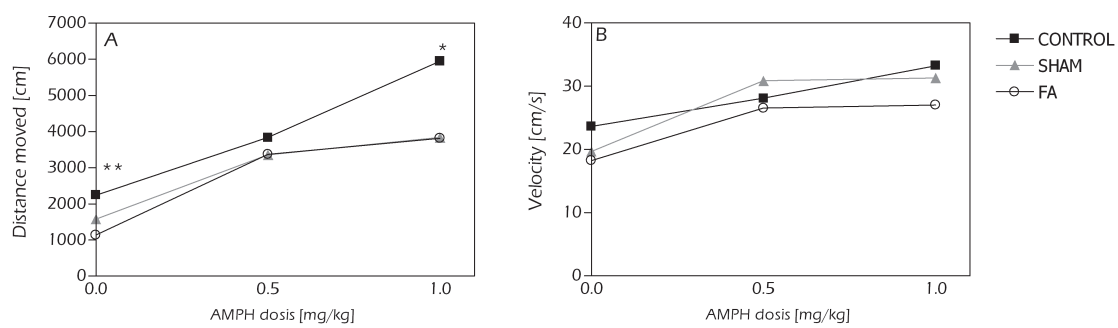

Figure 1. The effect of fetal asphyxia on basal and AMPH-induced motor behavior during open field testing (A) Mean distance moved (cm x 103) in open field. There was a significant effect of dose on distance moved. Under both basal and $1.0 \mathrm{mg} / \mathrm{kg}$ AMPH-stimulated conditions, SHAM and FA animals showed a reduced distance traveled compared to control animals. There was no significant difference between the SHAM and FA group. Furthermore, no differences were found under $0.5 \mathrm{mg} / \mathrm{kg}$ AMPH conditions between groups. (B) Mean velocity $(\mathrm{cm} / \mathrm{s})$ during open field task. There was a significant effect of dose on the velocity. No significant differences in velocity were found between the different groups. Data are expressed as mean + SEM. $N=10$ animals per group. (MANOVA + Duncan; * $p<0.05$ and ${ }^{* *} p<0.01$ ) 


\section{Nigral and striatal volumes}

As illustrated in figure $2 \mathrm{~A}$, FA resulted in a reduction of the mean volume of the SNC when compared to control (Post-hoc Bonferroni; $p<0.001 ;-32 \%$ ) as well as SHAM animals (Post-hoc Bonferroni; $p=0.003 ;-16 \%$ ). Post-hoc testing indicated that the mean volume in SHAM group was smaller than in the control group (Post-hoc Bonferroni; $p=0.005 ;-16 \%$ ). In contrast to the changed nigral volumes, the striatal volumes were not affected by either FA or SHAM procedure (figure 2B).

\section{TH-positive cells in substantia nigra pars compacta}

Results of the stereological analysis of TH-positive cells in the SNC are shown in figures 2C-D. There was a profound loss of TH-positive neurons as a result of FA when compared to both the control (Post-hoc Bonferroni; $p<0.001$; -39\%; figure $2 \mathrm{C}$ ) and the sham-operated group (Post-hoc Bonferroni; $p=0.035 ;-17 \%$ ). SHAM also significantly lowered the number of $\mathrm{TH}$-positive cells as compared to the control group (Post-hoc Bonferroni; $p=0.006$; -22\%). Additionally, the density of TH-positive neurons in SNC was decreased in FA group (Post-hoc Bonferroni; $p=0.01 ;-19 \%$ ) and in the SHAM group (Post-hoc Bonferroni; $p=0.03 ;-11 \%$; figure 2D) in comparison with the control animals. However, in this case no significant difference was found between the SHAM and the FA group. See figures 3A-C for representative photographs of the $\mathrm{TH}$-stained sections of the SNC.

\section{TH-immunoreactivity in striatum}

As illustrated in figure 2E, both the FA insult (Post-hoc Bonferroni; $p<0.001$; $23 \%$ ) and the sham-operation (Post-hoc Bonferroni; $p=0.001 ;-18 \%$ ) caused a profound loss in TH-immunoreactivity in the striatum in comparison with the control group. There was no significant difference between the SHAM and FA animals. See figures $4 \mathrm{~A}-\mathrm{C}$ for representative photographs of $\mathrm{TH}$-stained sections of the striatum. 

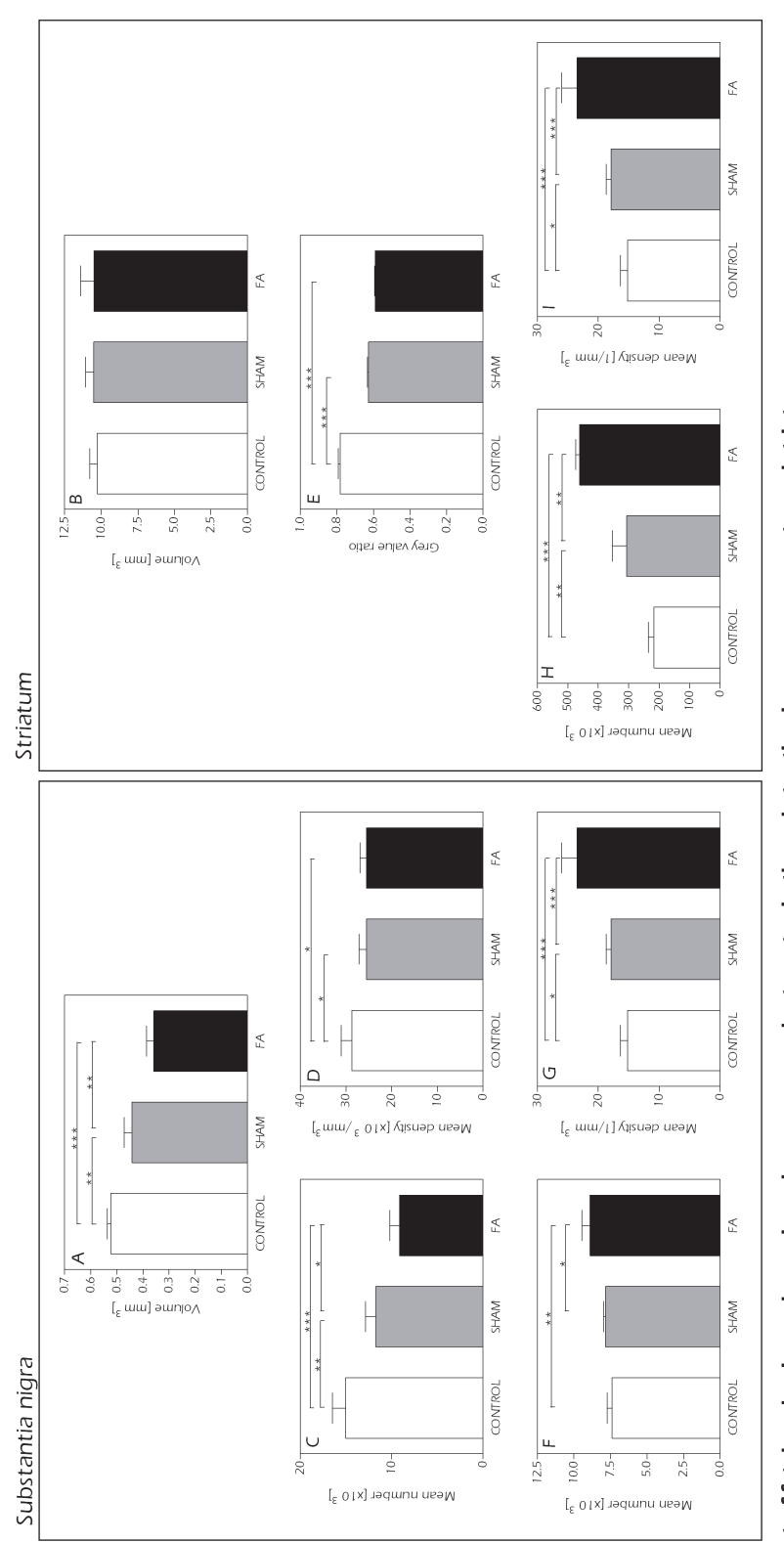

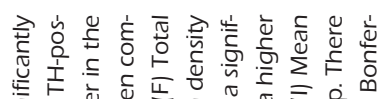

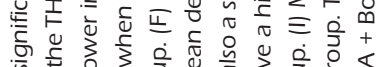

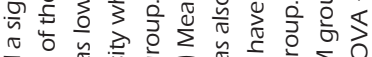

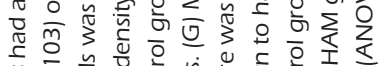

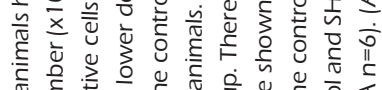

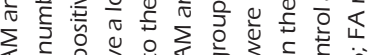

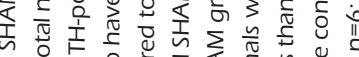

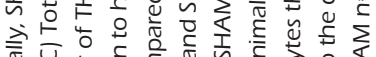

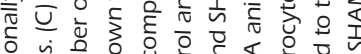

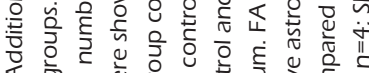

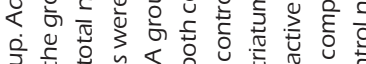
₹

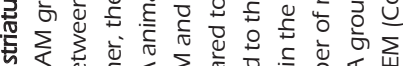

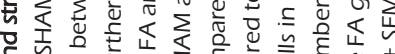

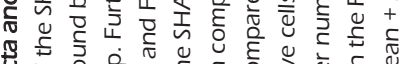

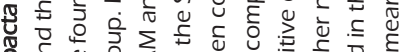

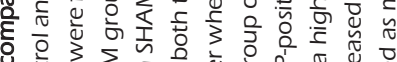

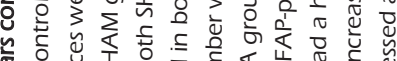

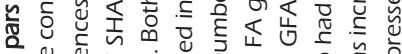

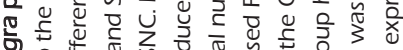

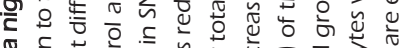

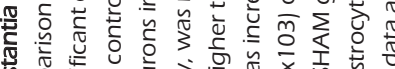

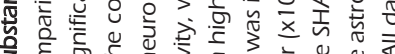
जू

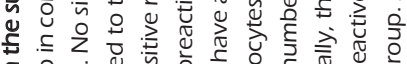

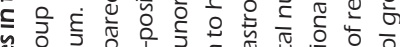

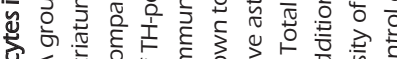

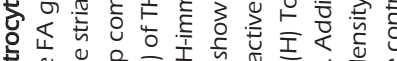

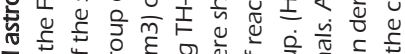

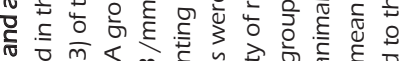

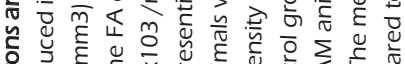

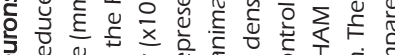

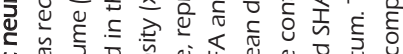

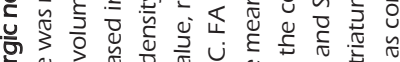

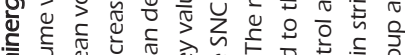

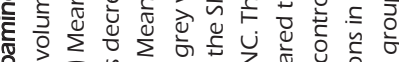

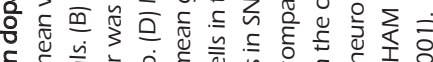

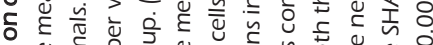

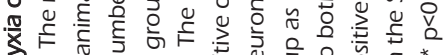
空讨

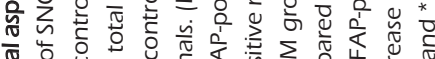

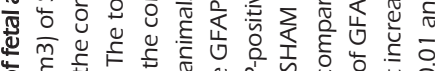

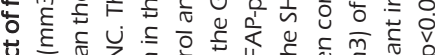

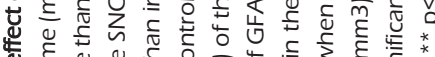

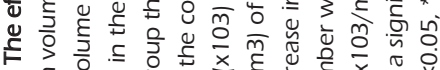

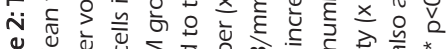

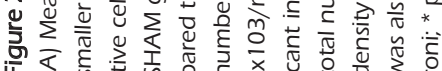



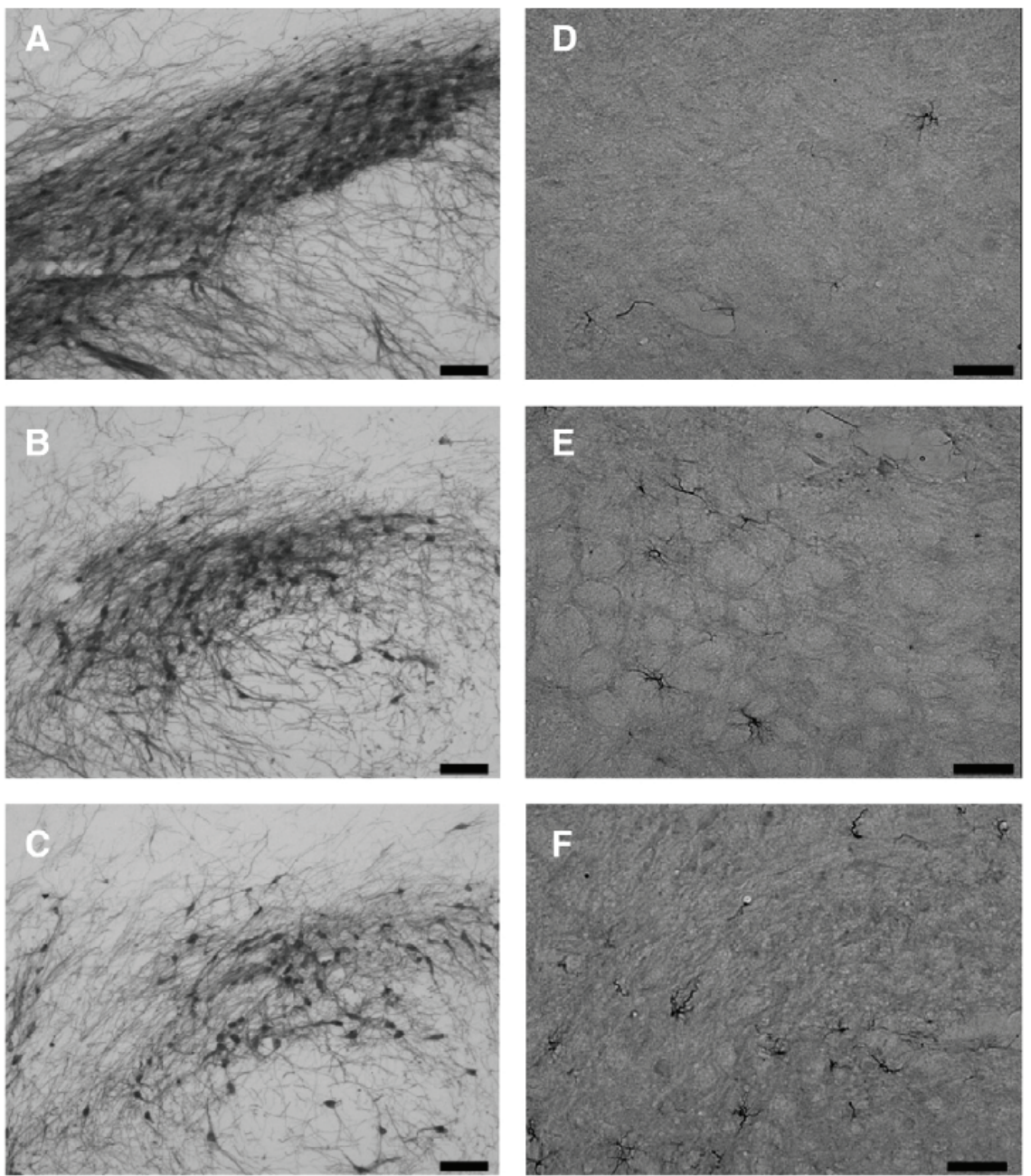

Figure 3: Photographs of TH- and GFAP-stained sections of the SNC

Representing photographs of TH-stained sections (magnification $4 x$ / scale bar $=200 \mu \mathrm{m}$ ) of the SNC of a control animal (A), a sham-operated animal (B) and a fetal asphyxic animal (C). Note the smaller size of the SNC in the fetal asphyxic animal when compared to both the control and SHAM animal. Representing photographs of GFAP-stained sections (magnification 20x / scale bar $=50 \mu \mathrm{m}$ ) of the SNC of a control animal $(D)$, a sham-operated animal (E) and a fetal asphyxic animal (F). Note the higher number of reactive astrocytes in the FA animal compared to both the control and SHAM animal. All photographs were taken at the same frontal level at which the optic tract divides the ventral tegmental area from the substantia nigra. 

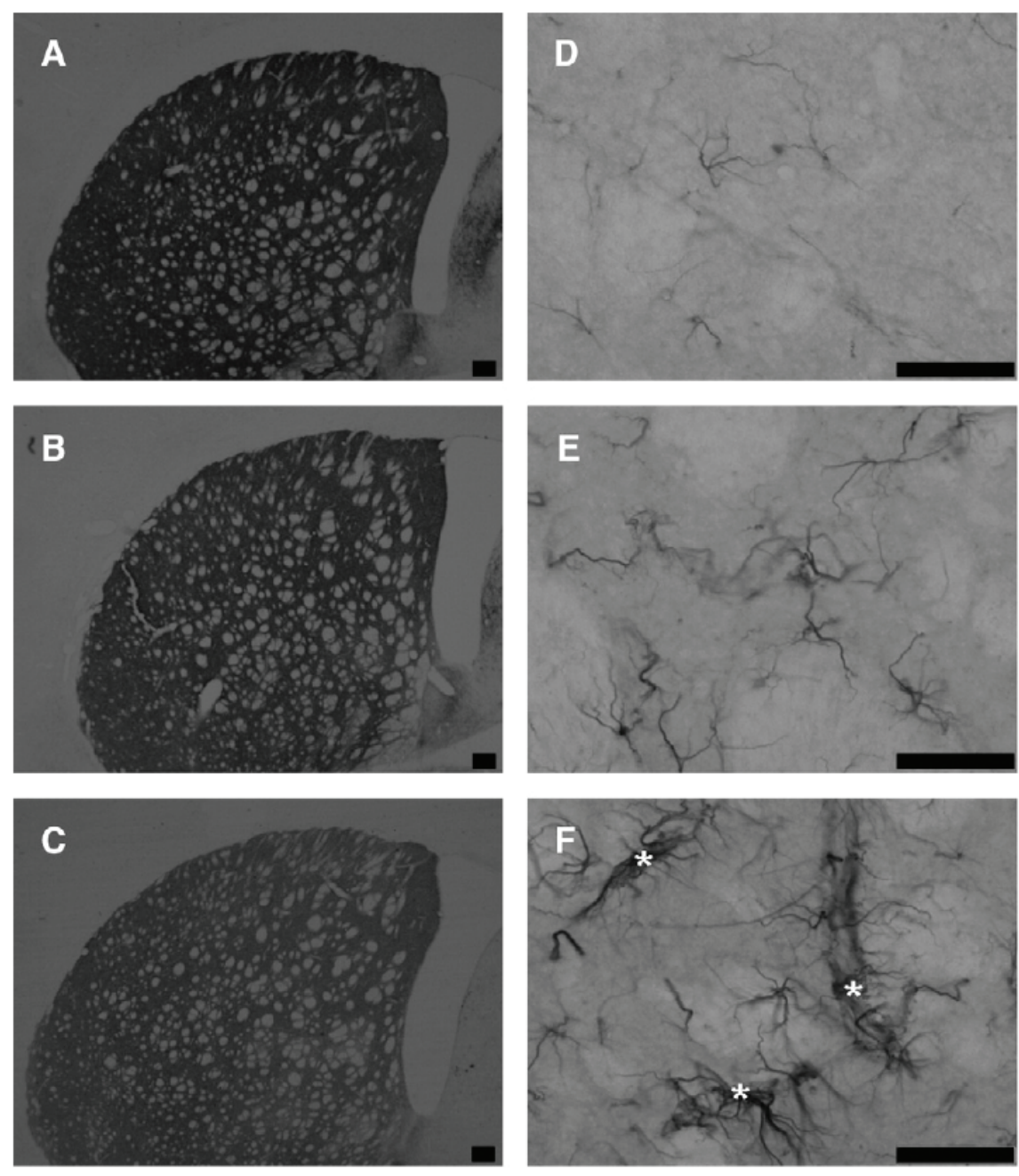

Figure 4: Photographs of TH- and GFAP-stained sections of the striatum

Representing photographs of TH-stained sections (magnification $2 x$ / scale bar $=200 \mu \mathrm{m}$ ) of the striatum of a control animal (A), a sham-operated animal (B) and a fetal asphyxic animal (C). Note the TH-immunoreactivity was lower, as shown by the lower grey value, in the FA animal than in both the control and SHAM animal. Representing photographs of GFAP-stained sections (magnification 40x / scale bar $=50 \mu \mathrm{m}$ ) of the striatum of a control animal (D), a sham-operated animal (E) and a fetal asphyxic animal (F). Note the intense the intense GFAP-positive and hypertrophied astrocytes in the striatum after fetal asphyxia $\left({ }^{*}\right)$. All photographs are taken at the same level. 


\section{GFAP-positive cells in substantia nigra pars compacta}

Figures 2F-G depict the results of the stereological analysis of GFAP in the SNC. FA animals showed significantly more reactive astrocytes in the SNC as compared to both the control (Post-hoc Bonferroni; $\mathrm{p}=0.003 ;+20 \%$; Figure $2 \mathrm{~F}$ ) and the SHAM group (Post-hoc Bonferroni; $p=0.04 ;+13 \%$ ). Additionally, a significant increase was found in the density of GFAP-positive astrocytes in the FA animals in comparison to control (Post-hoc Bonferroni; $p<0.001$; +54\%; figure 2G) and SHAM animals (Post-hoc Bonferroni; $p<0.001 ;+31 \%$ ). This density was also higher in the SHAM group than in the control group (Post-hoc Bonferroni; $p=0.014 ;+18 \%)$. See figures 3D-F for representative photographs of GFAPstained sections of the SNC.

\section{GFAP-positive cells in striatum}

Results of the stereological analysis of GFAP-positive cells in the striatum are given in figures $2 \mathrm{H}-\mathrm{I}$. The data demonstrated a significant increase in the total number of reactive astrocytes throughout the striatum in FA group as compared to both the control group (Post-hoc Bonferroni; $\mathrm{p}<0.001 ;+151 \%$; figure $2 \mathrm{H}$ ) and the SHAM group (Post-hoc Bonferroni; $\mathrm{p}<0.001 ;+54 \%$ ). SHAM also increased the total number of astrocytes in comparison with the control group (Post-hoc Bonferroni; $\mathrm{p}=0.002 ;+97 \%$ ). In addition, the mean density of reactive astrocytes was higher in the FA group when compared to the control (Post-hoc Bonferroni; $\mathrm{p}<0.001$; + 104\%; figure 2I) and the SHAM group (Post-hoc Bonferroni; $p<0.001 ;+71 \%$ ). Moreover, post-hoc testing demonstrated that the mean density was higher in the SHAM group than in the control group (Posthoc Bonferroni; $\mathrm{p}<0.014 ;+33 \%$ ). Of note, astrocytes in the FA group appeared to be more darkly stained and to possess thicker processes (see figures 4D-F).

\section{Cerebral and ventricular volumes}

The volume estimates of the lateral ventricles and total brain are shown in figures 5A-C. FA significantly enlarged the ventricular volumes compared to both the control (Post-hoc Bonferroni; $p=0.014 ;+52 \%$; figure $5 A$ ) and SHAM group 
(Post-hoc Bonferroni; $p=0.036 ;+38 \%$ ). Total brain volumes did not differ between groups (figure 5B). For that reason, the percentage ventricle of total brain volume was higher in FA animals than in the control (Post-hoc Bonferroni; $p=0.017$; $+44 \%$; figure $5 \mathrm{C}$ ) and the SHAM animals (Post-hoc Bonferroni; $p=0.049 ;+31 \%)$. White matter and cortical grey matter volumes were measured as well (Figure 5D-E). FA significantly decreased the white matter volume (Post-hoc Bonferroni; $p=0.03 ;-14 \%$; figure 5D) as well as the cortical grey matter volume (Post-hoc Bonferroni; $\mathrm{p}=0.009 ;-12 \%$, figure $5 \mathrm{E}$ ) in comparison to control animals. SHAM caused no significant differences in either white matter or cortical grey matter volume. Representing photographs are given in figures 6A-C. Like shown in the photograph, the ventricles are asymmetric with one ventricle being larger than the other one. This was the case in all FA animals. Control and SHAM animals showed no asymmetry.
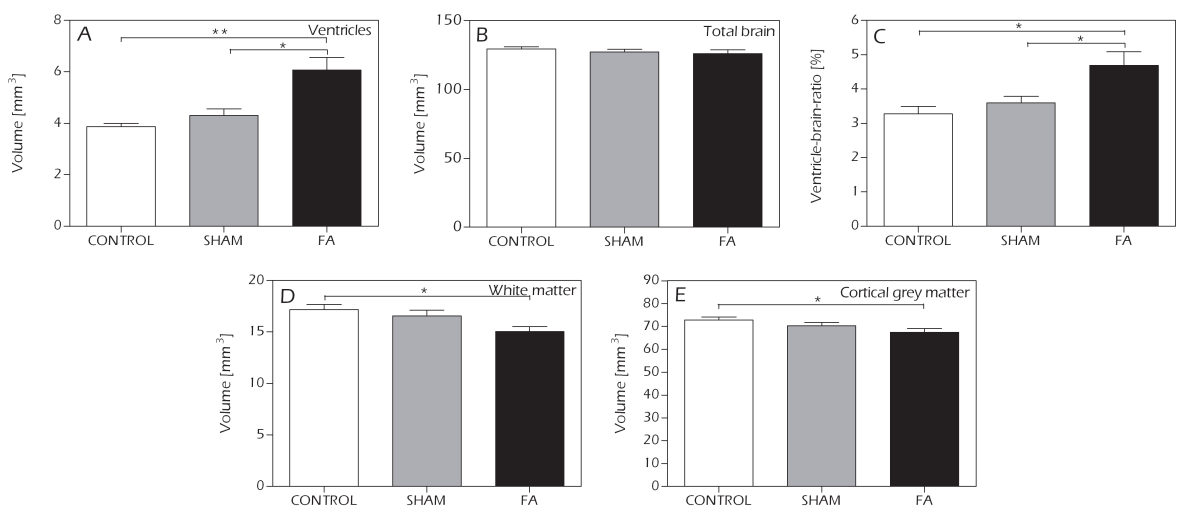

Figure 5: The effect of fetal asphyxia on the ventricular and total brain volume

(A) Results of the ventricular volume (mm3). Mean volume of the lateral ventricles was higher FA group compared to both the control and SHAM group. (B) Results of the total brain volume. No significant differences were found in total brain volume. (C) Results of the percentage ventricle of the total brain volume. Because of equal brain volumes, this percentage was higher in the FA animals compared to the control and SHAM animals. (D) Results of the white matter volume (mm3). Mean white matter volume was significantly lower in the FA group than in the control group. (E) Results of the cortical grey matter volume measurements (mm3). The cortical grey matter volume was significantly smaller in the FA group in comparison to the control group. Data are expressed as mean + SEM (Control $n=4 ;$ SHAM n=6; FA n=6). (ANOVA + Bonferroni; * $p<0.05)$. 

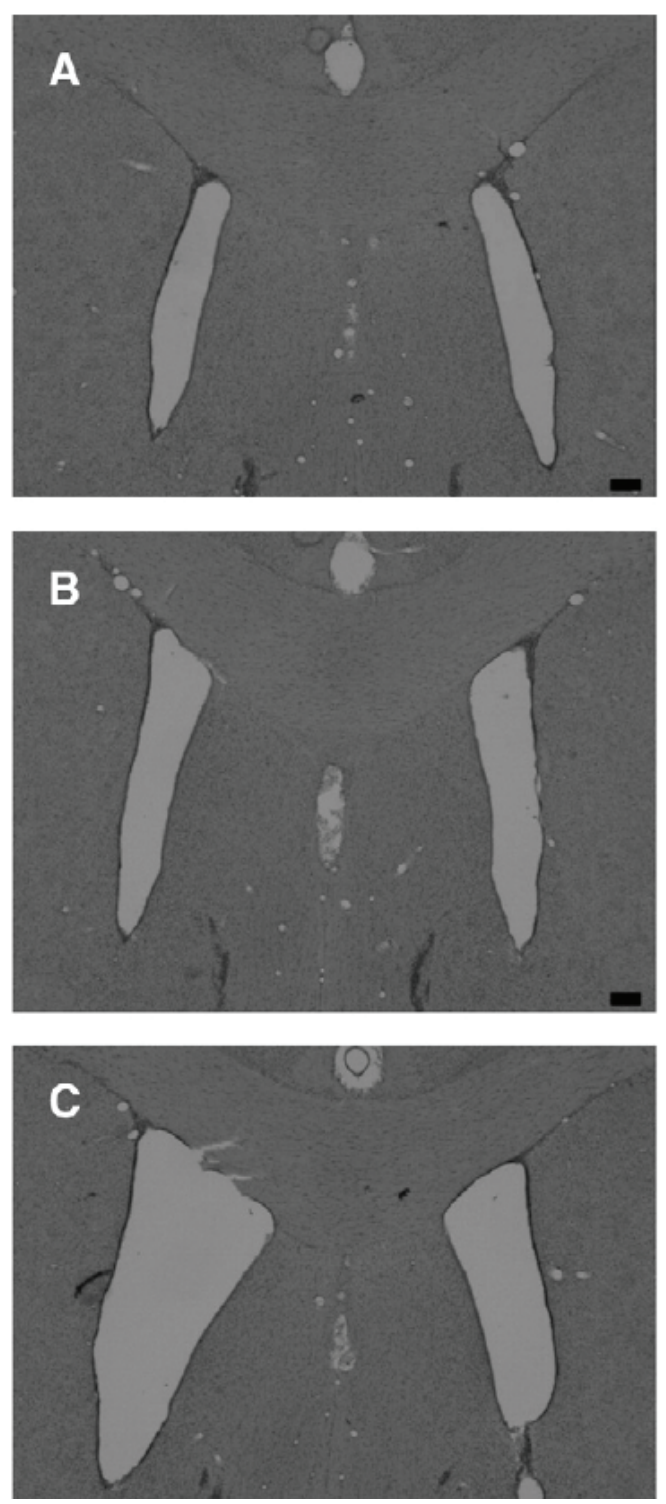

Figure 6: Photographs of the lateral ventricles

Representing photographs (magnification $2 x$ / scale bar $=$ $200 \mu \mathrm{m}$ ) of the lateral ventricles of a control animal (A), a sham-operated animal (B) and fetal asphyxic animal (C). Note the enlarged ventricles in the FA animals compared to both the control and the SHAM animal. All photographs were taken at the same level. 


\section{Discussion}

The present study shows that FA can produce long-term impairments in the dopaminergic nigrostriatal system and dopamine-mediated motor behavior in 6-month-old rats. At behavioral level, the results demonstrate that rats born after a FA insult show a reduction in basal as well as $1.0 \mathrm{mg} / \mathrm{kg}$ AMPH-induced locomotor activity during open field testing. Furthermore, immunohistochemical analysis revealed a diminished number and density of $\mathrm{TH}$-positive neurons in the SNC as well as a decreased TH-immunoreactivity in the striatum. These histological changes were accompanied by an increase in the number of GFAP-positive astrocytes in the SNC as well as the striatum. Although, sham-operated animals showed a similar impairment in locomotor behavior, the neuron loss was graded, being more severe in the FA group than in the SHAM group. Additionally, FA animals showed ventriculomegaly as well as smaller white matter and cortical grey matter volumes.

\section{Fetal asphyxia impairs dopamine-mediated locomotion.}

Previous research showed that perinatal asphyxia can reduce the striatal dopamine release under both basal and $\mathrm{AMPH}$-stimulated conditions in 6month-old rats [180]. For that reason, both basal and AMPH-induced locomotor activity were measured during open field testing. In normal animals, AMPH increases locomotion by preventing the reuptake of dopamine into the presynaptic cell and enhancing the release of this neurotransmitter from presynaptic cells. Together, these two processes augment the amount of dopamine available in the synaptic cleft, increasing dopaminergic transmission by stimulation of postsynaptic dopamine receptors [194]. In accordance, the present study demonstrated a significant effect of AMPH dose on both distance moved and the velocity. Our experiments showed that FA decreased the total distance moved under basal conditions during open field testing. Furthermore, FA and SHAM animals were not able to increase the distance moved in the same extent as in the control animals in response to $1.0 \mathrm{mg} / \mathrm{kg} \mathrm{AMPH}$. The velocity under either basal conditions or AMPH stimulation did not significantly differ between groups. In conclusion, FA only affected the total distance moved and 
not the movement velocity.

Similar deficits in dopamine-mediated locomotor behavior have been reported before in other models of developmental brain hypoxia and/or ischemia [166, $195,196]$. Those models are based on perinatal insults, whereas our model is based on a fetal event. The work of Loidl et al.(2000) demonstrated motor hypoactivity in 5-month-old rats that suffered from SPA. Likewise, Chen et al. (1995) found a decrease in basal locomotion, motility and rearing following 20 minutes of SPA in 4-week-old rats. In that same study, AMPH was not able to increase these parameters in the asphyxic animals. In a guinea pig model of a global anoxia during birth (2 minutes), Vaillancourt and Boksa (2000) showed reduced $\mathrm{AMPH}$-induced locomotion 3 months after the insult compared to control animals. On the other hand, Cai and colleagues (1999) demonstrated that 30 minutes of fetal asphyxia at E17 increased locomotor activity on postnatal days 13 and 15. A similar increase in locomotor activity was seen between postnatal days 12 and 18 in a bilateral carotid artery occlusion model at P4 [197]. In both studies this hyperactivity seemed transient, since it was no longer present after 3 weeks. Furthermore, 19-20 minutes SPA caused a decrease in locomotion, while a milder form (15-16 minutes) caused an increase in locomotion. These dissimilarities may be explained by either timing, i.e. developmental stage of the brain, or severity of the insult.

\section{Fetal asphyxia causes dopaminergic changes in the nigrostriatal pathway}

In the present study, both SHAM and FA decreased the number and density of dopaminergic neurons in the SNC. Nevertheless, the number of TH-positive cells was significantly lower in the FA group than in the SHAM group. These observations are in agreement with previous reports. Both short and long-term decreases in the number of $\mathrm{TH}$-positive cells were reported in other developmental asphyxic models. Early postnatal bilateral carotid occlusion followed by 15 minutes of hypoxia resulted in a loss of TH-positive cells in the SNC in neonatal (postnatal days 9 and 21 ) as well as adult rats (postnatal days 56 and 84) $[181,182,197]$. Moreover, the Rice-Vannucci model as well as severe episodes of perinatal asphyxia (19-20 minutes) also reduced the number of $\mathrm{TH}$-positive 
cell bodies in the SNC in 4-week-old rats $[178,181]$. Besides in the SNC, both SHAM and FA also caused reductions in TH-immunoreactivity in the striatum in contrast to control animals. The decreased TH-immunoreactivity in the striatum could be easily explained by a reduced number of $\mathrm{TH}$-positive cells in the SNC, given that the SN projects to the striatum.

Since the sham-operation, without FA procedure, altered both dopamine-related behavior and biochemistry, we suggest that the sham-operation also causes a mild form of fetal asphyxia. It seems plausible that the interventions (i.e. laparotomy and extra-abdominal exposure of the uterine horns for 75 minutes) might cause changes in uterine blood flow, thereby affecting the oxygenation to the fetuses. Although the literature is very contradictory, part of the changes may also be caused by the anesthesia (e.g. combination of xyalzine and ketamine. According to Young et al and Rudin et al, a single exposure to ketamine can induce apoptotic neurodegeneration in different regions of the developing brain $[198,199]$. Though, recently Ferro et al. found that the combination of ketamine and xylazine can have a neuroprotective effect as well [200]. They observed protection at histological as well as behavioral level in two different rat models for Parkinson's disease (e.g. 6-OHDA model and MPTP model). Furthermore, it is important to note that the results of both groups (SHAM and FA) are not completely similar. The neuron loss was graded, being more severe in the FA group than in the SHAM group.

The impairments in motor behavior following fetal asphyxia may be explained by the reduction in TH-positive cells in the SNC as well as a decreased immunoreactivity in the striatum, as demonstrated in this study. It is known that the dopaminergic systems in the basal ganglia play a central role in the control of motor behavior in the rat. In particular the nigrostriatal pathway, which arises from the SNC A9 cell group and projects to the striatum thereby modulating the striatal neurons, is of great importance [reviewed in [183, 201]]. Therefore, an insufficient dopamine transmission in the nigrostriatal dopamine pathway would generally lead to a reduction of movement and impulses. Furthermore, it is known that the basal ganglia seem extremely sensitive to oxygen deprivation [202]. Another possible explanation for the changes in locomotor behavior could be post-synaptic impairments, like lower receptor numbers, because AMPH was not able to improve locomotion in the FA animals to the 
same extent as in the control animals. The hypoactivity observed may also be linked to previous observations in different developmental models, in which morphological nigral and striatal alterations were found. SPA caused a reduction in dopamine utilization in the striatum and the SN [203]. The same model also showed a decreased number of $D 1$ receptors in the $S N$ at the age of 4 weeks [179]. Furthermore, 6-month-old rats exposed to perinatal asphyxia showed a reduced striatal dopamine release under both basal and AMPH-stimulated conditions [180]. The same changes in dopamine release were found in the basal ganglia in 4-week-old perinatal asphyxic rats [204]. In addition, perinatal asphyxia caused a decrease in the number and length of $\mathrm{TH}$-positive processes in organotypic cultures from the $\mathrm{SN}$ and striatum, especially from secondary or higher level branching $[177,205,206]$.

The loss of dopaminergic cells in the SNC and the loss in TH-immunoreactivity in the striatum at the age of 6-months will most likely have a developmental origin. The possibility that a fetal asphyxic insult at E17 interferes with cell division of nigral precursor cells in the subventricular zone (SVZ) seems unlikely, since $[3 \mathrm{H}]$ thymidine and $\mathrm{BrdU}$ experiments have shown that these precursors undergo mitosis between E12 and E15 [184-187]. However, between E15 and E18 nigral precursor cells migrate from the SVZ to the SN [188, 189]. For that reason, it might be possible that a fetal insult at this specific time point (E17) causes an incomplete functional incorporation, impeding the attainment of a normal number of dopaminergic cells in the SN. Further, migrating nigral precursor cells may be more prone to cell death during fetal asphyxia. In support of this idea, Oo and colleagues (1995) showed that early hypoxic-ischemic injury induced apoptotic cell death in the dopaminergic neurons of the SN. These processes could be responsible for the diminished number of TH-positive cells in the adult SN. Evidently, these interpretations must be validated by further studies.

\section{Fetal asphyxia causes nigrostriatal astrogliosis}

The loss of TH-positive cells in the SNC and the loss in TH-immunoreactivity in the striatum were accompanied by an increase in the number GFAP-positive cells (astrocytic hyperplasia). In addition, the astrocytes appeared to be more 
darkly stained and to possess thicker processes (astrocytic hypertrophy). These are characteristics of astrogliosis, a common neurocellular event in brain pathology as a response to many diseases and/or injury [reviewed in [207, 208]]. Similar increases in GFAP-positive cells were reported a few times before in different animal models of developmental asphyxia. Cebral et al. (2006) demonstrated striatal astrogliosis in 6-month-old rats born after SPA, while Benjelloun et al (1999) showed proliferation of astrocytes apparent 24h after a left common carotid artery occlusion at P7. Futhermore, Ezquer and collegues (2006) found an increased GFAP expression throughout the SN at $48 \mathrm{~h}$ after severe hypoxia in a 7-day-old rat pup. In our model, the astrogliosis seems persistent, since it was still apparent 6 months after the insult.

The precise cause and function of astrogliosis in still not known, but recent data indicate that this pathological process is associated with inflammatory processes and the production of reactive oxygen species, two events linked with asphyxic insults [209-211]. For quite some time, it has been thought that astrogliosis was a response to neuronal death to replace the lost neurons. Recent evidence suggests that the process of astrogliosis can induce detrimental effects. Glial tissue can interfere with normal functioning of neuronal circuits by inhibiting regeneration and causing electrical instability [212]. These harmful processes might contribute to malfunctioning of the striatum and consequently the nigrostriatal pathway, seen in the locomotor behavior in our animals. However, besides its detrimental effects, astrogliosis is may actually be beneficial as well. Reactive astrocytes are believed to reestablish a protective barrier, namely the glial limiting membrane. In this way, they may assist repair/recovery of the neuronal tissue by stabilizing the surrounding environment, i.e. by producing neurotrophic factors promoting neuronal survival [212, 213]. In addition, neurons are highly dependent on the functioning of astrocytes for nutrition and maintenance of oxygen supply [214]. For that reason, a rise in reactive astrocytes due to hypoxic and ischemic insult may be important. 


\section{Fetal asphyxia causes ventriculomegaly, white matter loss and atrophy of the cerebral cortex}

To asses brain atrophy, cerebral and ventricular volumes were measured in our model. The present study showed that FA significantly enlarged the volumes of the lateral ventricles, while both the striatal and total brain volume remained unchanged. In the assumption that other brain regions have to compensate for the dilatation of the lateral ventricles, cortical grey matter and white matter volumes were measured as well. Both the cortical grey matter and the white matter volumes were significantly smaller in the FA animals. Similar findings were reported in others rodent models of neonatal hypoxia and/or ischemia. Ment et al demonstrated progressive cerebral ventriculomegaly, associated with a decreased subcortical white matter, a smaller corpus callosum and a decreased cortical volume in a rat model for chronic sublethal hypoxia at postnatal day 3 [215]. Turner et al. also found ventriculomegaly in a similar mouse model [216]. Ventricle enlargements, atrophy of the cortex and white matter loss are abnormalities found in imaging studies of human asphyxic term and preterm infants as well $[49,217,218]$. Ventriculomegaly is probably secondary damage that represents both cortical and white matter damage [219]. White matter loss can be caused by an incomplete vascular supply or an impaired regulation of the cerebral blood flow inadequate to perfuse the white matter. The white matter is susceptible for hypoxia-ischemia because of the high metabolic activity needed for the myelination during development [219]. The locations of the highest glucose uptake within the neonatal brain correlate with the locations of the most advanced myelination. Another reason can be that the pre-oligodendrocytes forming the white matter are extremely vulnerable for hypoxia-ischemia [220].

Available clinical data suggest that infants with ventriculomegaly of different etiologies have a higher frequency of cognitive and motor handicaps [221]. Probably because ventriculomegaly is associated with reductions in white matter volume that can have profound effects on hemispheric connectivity. An important remark to be made is that all FA animals showed asymmetrical bilateral ventricle enlargement. Bilateral asymmetrical ventriculomegaly is more often 
associated with developmental delay and postnatal neurological disease compared to symmetrical and unilateral enlargements [222].

\section{Implications}

The findings of this study may be important in understanding the pathological mechanisms of FA. Although direct implications for the human fetus cannot be correctly extrapolated yet, this study shows that FA can cause subtle but longlasting changes in the brain. These changes may be relevant for better understanding the pathogenesis of different neurological disorders. For example, white matter loss associated with ventriculomegaly forms a neuropathological substrate for both cognitive and motor deficits later in life [221]. Furthermore, asphyxic insults during development may be a factor contributing to the development of neurodegenerative diseases [166, 180]. FA might make the brain more prone to neurodegeneration later in life. Loss of neurons due to early disruptions in brain development (e.g. fetal asphyxia; first hit) may produce a long-term vulnerability to a second or third hit (e.g. normal aging or other risk factors) for the development of disorders, like Alzheimer's disease or Parkinson's disease. For example, this study demonstrated the loss of dopaminergic neurons related to a decrease in locomotor activity. It seems plausible that fetal asphyxia could accelerate the onset and progression of age-related loss of dopaminergic neurons, enhancing the vulnerability to develop parkinsonian symptoms [223].

\section{General conclusion}

The present study is the first one to demonstrate that FA causes long-term deficits in dopamine-mediated locomotion associated with the loss of dopaminergic neurons in the SNC. These alterations were related to nigrostriatal astrogliosis. Additionally, FA animals showed ventriculomegaly probably caused by the loss of white matter as well as cortical grey matter volume. This study shows that FA can cause subtle but long-lasting changes in the brain that may be relevant to the pathophysiology of different neurological disorders. 

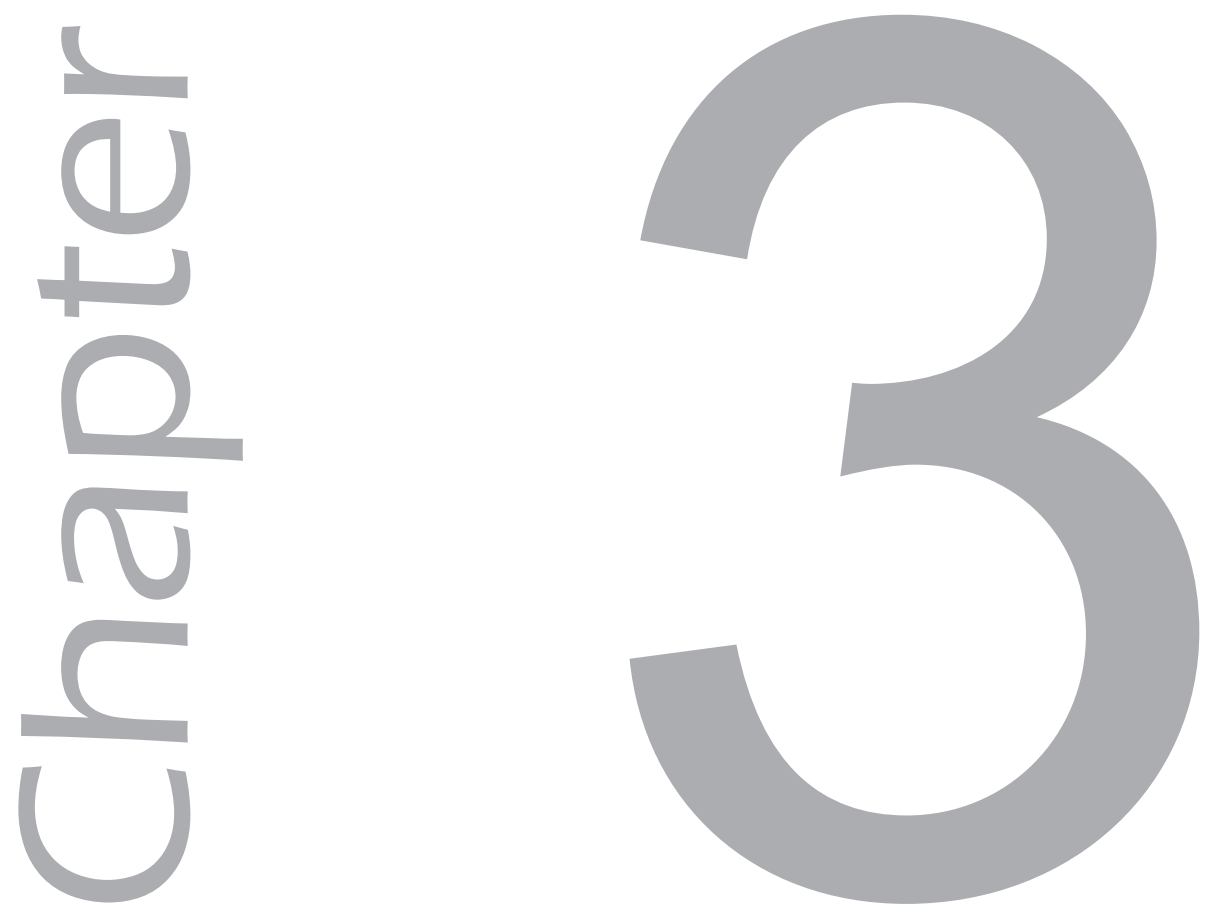

\section{Fetal asphyxia leads to a decrease in dorsal raphe serotonergic neurons}

E. Strackx, D.L.A. Van den Hove, H.P. Steinbusch, J. Prickaerts, J.S.H. Vles, C.E. Blanco, H.W.M. Steinbusch and A.W.D. Gavilanes

Developmental Neuroscience (2008), 30(5): 358-366 


\begin{abstract}
The aim of the present study was to determine the effects of fetal asphyxia (FA) on anxiety and serotonergic neurons in young adult and middle-aged rats. FA was induced at E17 by clamping the uterine circulation for 75 minutes. Anxiety-related behavior was tested in an open field and design-based stereology was used for counting serotonergic (5-HT) neurons in the dorsal raphe nucleus (DRN). The open field revealed increased anxiety in the 19-month-old FA rats in comparison to control animals. No significant differences were found in DRN 5-HT neuron numbers at 6 months. At 19 months, however, FA significantly lowered the mean density and volume of 5-HT neurons in the DRN as compared to controls. Further, an age-related reduction was found in the total number, the mean density and the mean volume of 5-HT neurons within the FA group. In conclusion, FA is associated with increased anxiety and age-related changes in 5-HT immunohistochemistry within the DRN. These results support the notion that asphyctic insults during critical periods of brain development could predispose to serotonergic abnormalities and anxiety deficits in adulthood.
\end{abstract}


sphyxic insults on the developing brain, whether pre-, peri-, or post-
natal, are the most important cause of neonatal mortality and mor-
bidity [3, 7, 161]. They can cause dramatic neurological deficits in
children, including seizures, mental retardation and motor impairments like cerebral palsy [24, 224, 225]. In addition, developmental asphyxia may contribute to the development of psychiatric disorders as well as neurodegenerative disorders in adult life $[162,164,166]$.

Various animal studies have previously examined the consequences of term perinatal asphyxia during early adulthood. The main findings of those studies were that severe perinatal asphyxia (19-20 minutes) is associated with impaired locomotor activity, decreased anxiety-related behavior as well as learning deficits in later life $[101,166,226]$. Little is known about the long-term consequences of an asphyxic insult in middle-aged and aged animals. Previous research in our group showed impaired memory performance in 18-month-old perinatal asphyxic rats compared to control animals [57]. Likewise, Weitzdoerfer et al demonstrated lower cognitive function in 2-year-old perinatal asphyxic rats [102]. These changes were associated with higher hippocampal levels of the serotonin (5-HT) transporter. The same group also found increased anxiety levels in the same perinatal animal model [103]. Cai et al reported an altered hippocampal nitric oxide synthase (NOS) system and learning deficits in young rats previously subjected to fetal asphyxia (FA) [93]. Recently, our group has shown nigro-striatal dopaminergic disruption and motor behavioral deficits in aged rats previously subjected to FA [227]. Nonetheless, the long-term effects of an asphyxic event during the fetal period in the aged rat are still unknown.

It is widely accepted that dysregulation of the 5-HT neurotransmitter system plays an important role in the pathophysiology of affective disorders in adulthood [228]. The underlying mechanisms of these disorders are still unclear. One of the current thoughts is that a combination of specific functional and/or anatomical pathways and different 5-HT receptor subtypes may be responsible for different syndromes of anxiety and depression [229-231]. Moreover, it is known that 5-HT plays an important role in normal brain development, besides its a role as a neurotransmitter in the mature brain [232, 233].

The aim of the present study was to determine the long-term effects of FA on 
anxiety-related behavior and the 5-HT circuitry of the DRN in adult (6 months) and middle-aged ( 19 months) rats. This study only focuses on 5-HT in the DRN because this structure is the main source of the serotonergic input to the forebrain and it is involved in the control of the affective state [234].

\title{
Materials and methods
}

\begin{abstract}
Animals
All experiments were approved by Animal Ethics Board of the University of Maastricht on animal welfare according to Dutch governmental regulations. All efforts were made to minimize the pain and stress levels and to minimize the number of rats necessary to produce reliable scientific data. Inbred date-pregnant Lewis rats were supplied on day 14 of gestation by the Animal Care Department of the University of Maastricht and housed individually in its facilities. The rats were kept under standard laboratory conditions with food and water given ad libitum, $21 \pm 2^{\circ} \mathrm{C}$ ambient temperature, a $12 \mathrm{~h}$ light/dark schedule (lights on at 07:00h) and background noise provided by radio.
\end{abstract}

\section{Fetal asphyxia procedure}

At embryonic day 17 (E 17), rats were anaesthetized by xylazine ( $1 \mathrm{mg} / \mathrm{kg}$ s.c.) and ketamine (50mg/kg i.m.). Dams were subjected to a midline laparotomy to expose both uterine horns. All procedures were performed within a controlled environment $\left(37^{\circ} \mathrm{C}, 80 \%\right.$ humidity, room air). FA was achieved by completely clamping both uterine and ovarian arteries with removable clamps. The uterine horns were kept wet, by rinsing them with $0.9 \%$ saline solution and dams were injected subcutaneous with $0.9 \%$ saline $(5 \mathrm{ml} / 100 \mathrm{~g}$ interscapularly) to prevent dehydration. After 75 minutes, the clamps were removed to allow perfusion again. The uterine horns were placed back and the abdominal wall was repaired. SHAM-operated rats underwent the same procedure except for clamping the arteries. The FA, SHAM-operated and control animals were fullterm vaginally delivered on E21-E22. Exclusively male offspring were used, because both morphological and behavioral evidence shows a differential 
vulnerability to a birth insult. A lesser impact is seen in the female gender, probably due to the protecting role of the estrogens [191, 192].

\section{Behavioral testing}

At 19 months, the number of subjects was reduced in the three experimental groups (control= from 10 to 4, SHAM= from 11 to 7, and FA= from 11 to 6) due to spontaneous mortality caused by aging. At that age, the animals were tested in the open field, a valid test to study anxiety-related behavior [235]. This test was conducted by placing the rat in the centre of a $100 \times 100 \mathrm{~cm}$ plexiglas, square box with a $30 \mathrm{~cm}$ high enclosure. The arena was subdivided into different zones: a central zone $(60 \times 60 \mathrm{~cm}), 4$ corner zones $(20 \times 20 \mathrm{~cm})$ and 4 wall zones $(60 \times 20 \mathrm{~cm})$. The movements were recorded for 5 minutes with a film-camera attached to a computer running the Ethovision ${ }^{\mathrm{TM}}$ programme (Noldus Equipment, Wageningen, The Netherlands). The mean time spent in the different zones was scored. All animals were subjected to one 5-minute trial a day for 4 consecutive days. The data of the 4 consecutive days were averaged.

\section{Perfusion and tissue preparation}

Rats were anaesthetized with sodium pentobarbital (60mg/kg i.p.) and transcardially perfused with $4 \%$ paraformaldehyde in $0.1 \mathrm{M}$ phosphate buffer $(\mathrm{pH}$ 7.6). Brains were dissected, post-fixed for $2 \mathrm{~h}$ and suspended in 15\% sucrose/0.1 M phosphate buffer for $48 \mathrm{~h}$. They were cut serially into $30 \mu \mathrm{m}$ thick coronal sections using a cryostate. DRN sections were made from bregma 6.80mm until $-8.80 \mathrm{~mm}$.

\section{Immunohistochemistry}

Sections were immunostained, in a free-floating manner, with rabbit anti-5hydroxytryptamine antibodies 1:30000; Steinbusch, Maastricht University, The Netherlands), followed by incubation with donkey anti-rabbit biotinylated secondary antibodies (1:800; Jackson Immunoresearch Laboratories, West Grove, USA). Sections were further processed using the avidin biotin complex tech- 
nique with 3,3-diaminobenzidine (DAB) to obtain a color reaction (Vector laboratories, USA). Afterwards, sections were mounted, air-dried, dehydrated in ascending ethanol concentrations and coverslipped with depex. Sections from control ( $n=4$ for each time point), SHAM ( $n=6$ for each time point) and FA ( $n=6$ for each time point) animals were processed simultaneously under the same experimental conditions. On additional sections, nissl stains were performed. Sections were dried, defatted with triton-X100 and stained with cresyl violet (0,01\%, 20 minutes).

\section{Analysis}

Design-based stereology was performed to count neurons in Nissl stained sections as well as 5-HT-stained sections, using a stereological computer microscopy system and Stereolnvestigator software (MicroBrightField, Williston, VT, USA). The DRN was delineated at a 1.25x magnification on live microscopic images displayed on a monitor. The DRN borders were delineated between bregma -6.80 and -8.80 according to Paxinos and Watson [236]. The delineation is demonstrated in figure 1.

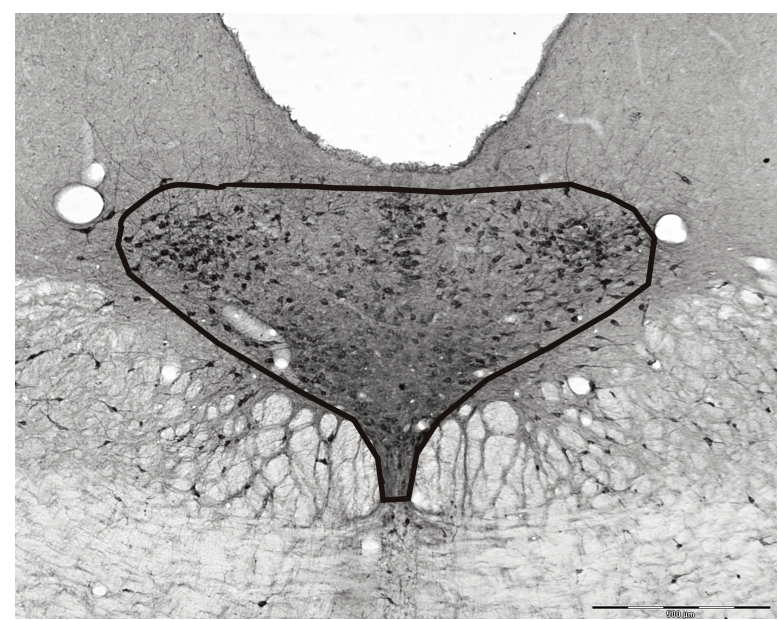

Figure 1: Representative photograph of a 5-HT stained section.

This photograph shows the delineation of the dorsal raphe nucleus in a 5-HT stained section of a control animal. 
Volume estimates were calculated, according to the Cavalieri's principle, by multiplying the average surface area with the section thickness, the number of sections, and a factor of eight since every eighth section was used /section sampling fraction $(\mathrm{ssf})=1 / 8)$. Cells were counted at magnification 50x with the optical fractionator method [193]. Briefly: counting frames were created by the software and placed at the intersections of a grid that had been randomly placed over the section. The counting frames were replaced systematically by stepwise movements in $\mathrm{x}$ - and $\mathrm{y}$-directions. The area of the counting frame relative to the area associated with the $x$ and $y$ steps gives the area sampling fraction (asf). The optical dissector height, along the z-axis, was determined by excluding the parts close to the slide and coverslip. The height of the optical dissector relative to the actual thickness results in the height sampling fraction (hsf). Cells were marked if they were positive and in focus within the counting area. Furthermore, the counting frames have forbidden lines to avoid edge effects. The estimated total number of positive cells $(N)$ was calculated from the number of cells counted (Q) according to the formula: $N=1$ /ssf * 1 /asf * 1 /hsf * $\mathrm{Q}$. Details on the stereological counting procedures are summarized in table 1.

Table 1: Details of the stereological procedure

\begin{tabular}{|c|c|c|c|c|c|c|c|c|}
\hline & Obj & B & $\mathrm{H}$ & $D_{x}$ & $D_{y}$ & $t$ & $\Sigma O^{-}$ & CEpred $_{[n]}$ \\
\hline NISSL, DRN & $50 x$ & 400 & 4 & 150 & 90 & 12,12 & 624 & 0,0400 \\
\hline 5-HT, DRN & $50 x$ & 400 & 4 & 150 & 90 & 12,26 & 512 & 0,0442 \\
\hline
\end{tabular}

NISSL, DRN, Neurons in the dorsal raphe nucleus; 5-HT, DRN, 5-HT-positive cells in the dorsal raphe nucleus; Obj, Objective used for counting; $B$, Base area of the unbiased virtual counting spaces in $\mu \mathrm{m}^{2} ; H$, Height of unbiased virtual counting spaces in $\mu \mathrm{m} ; D$, distance be tween the unbiased virtual counting spaces in directions $x$ and $y$ in $\mu \mathrm{m} ; t$, actual average section thickness measured after histological processing in $\mu \mathrm{m} ; \Sigma Q$-, average number of counted cells; CEpred[n], average predicted coefficient of error of the estimated total numbers of cells using the prediction method described by Schmitz and Hof (2005).

The mean cellular volume of all neurons was estimated with the nucleator method [193]. The midpoint of the nucleolus of each counted neuron was marked. Then the software randomly generated two mutually orthogonal lines through this midpoint. The boundaries of the cell body were identified at the intersections of these lines with the membrane. The volume can be calculated from the average of the third powers of these intercept measurements (I) (Vn $=4 \pi / 3 *(3 n)$. 
Immunoreactivity of 5-HT in the DRN was also measured by a semi-quantitative method. Photographs were taken within each section with the Olympus AX70 microscope connected to a digital camera (F-view; Olympus, Tokyo, Japan). To correct for potential variability in lighting conditions, all images were collected under identical conditions. Photographs were analyzed, using their corresponding average grey values (ranging from 0-black to 255-white), obtained with the NIH ImageJ software (http://rsb.info.nih.gov/ij/). Using a trial and error method, a threshold value was selected and kept the same for all measurements. Blood vessels, tissue out of focus or artifacts were excluded. Background IR was normalized within each sections by dividing the mean grey value by the average value of white matter, which has negligible $5 \mathrm{HT}$-staining.

\section{Statistics}

All data are represented as mean \pm standard error of means ( \pm SEM). All data were analyzed using a one-way analysis of variance (ANOVA) test, followed by post-hoc comparisons using Bonferroni test. Data from 6-month and 19-monthold animals were compared using a Student's t-test. The accepted level of statistical significance was $p<0.05$. All calculations were done using the Statistical Package for the Social Sciences (SPSS 12.0 software).

\section{Results}

\section{Fetal asphyxia increased anxiety-related behavior in 19- month-old rats}

The effect of FA on behavior in the open field is depicted in figure 2. FA rats spent more time in the corners (post-hoc Bonferroni; $\mathrm{p}=0.021 ;+31 \%$ ) and less time along the walls (post-hoc Bonferroni; $p=0.018 ;-51 \%$ ) of the open field as compared to control animals. In addition, the FA animals also spent significantly less time along the walls than the SHAM animals (Post-hoc Bonferroni; $p=0.047 ;-43 \%)$. Time spent in the center did not differ between groups. Addi- 
tionally, there was no significant effect on the movement velocity /data not shown).

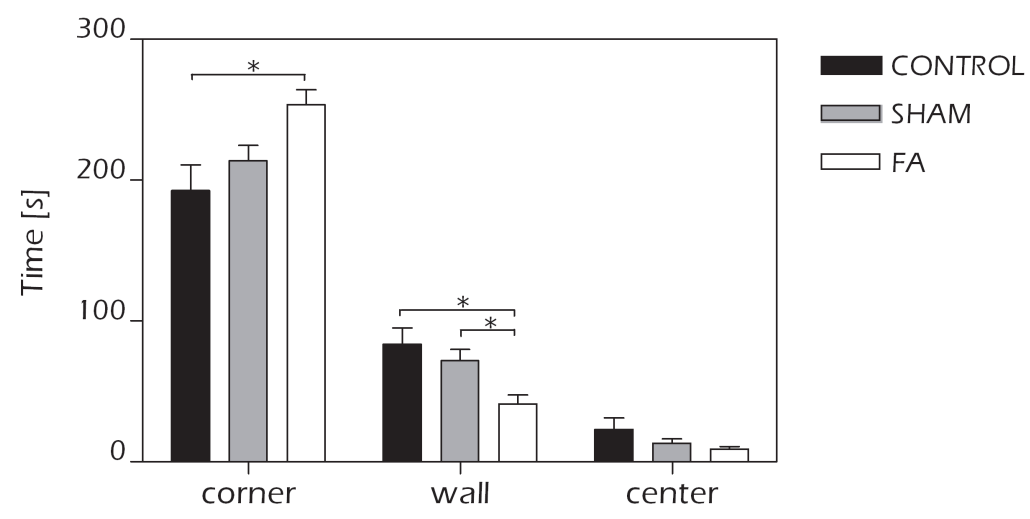

Figure 2: The effects of fetal asphyxia on open field, depicting the time spent in the different zones at the age of 19 months.

FA animals spent significantly more time in the corners and less time along the walls compared to both control and SHAM animals (control $n=4$, SHAM $n=7$ and FA $n=6$ ). Data represent mean + SEM. (ANOVA + Bonferroni; * $p<0.05)$

\section{Fetal asphyxia increased 5-HT-IR in DRN in 19-month-old rats}

Figure 3 illustrates the results of the 5-HT-IR measurements, represented as grey value ratios. No significant differences were observed at the age of 6 months. At 19 months, the FA group showed a profound increase in 5-HT-IR in the DRN in comparison to both the control (Bonferroni; $p<0.001 ;+56 \%$ ) and the SHAM group (Bonferroni; $p<0.001 ;+30 \%$ ). In addition, SHAM animals showed a higher 5-HT-IR than the controls (Bonferroni; $\mathrm{p}=0.009 ;+20 \%$ ).

\section{Fetal asphyxia did not affect the dorsal raphe volume}

Figure 4 depicts the volume estimates of the DRN. No significant volume differences found between groups at 6 or 19 months. Additionally, there were no volume alterations as a result of aging. 


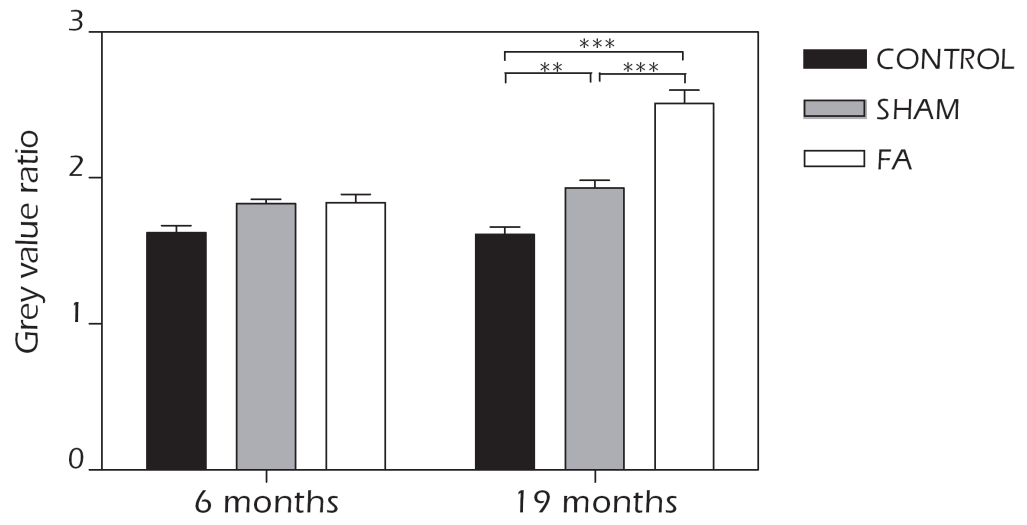

Figure 3: The effect of fetal asphyxia on 5-HT-immunoreactivity in the DRN.

(A) 5-HT-immunoreactivity, measured as grey values, at the age of 6 months. No significant differences were observed between groups. (B) 5-HT-immunoreactivity at the age of 19 months. Both SHAM and FA caused an increase in 5-HT-immunoreactivity in the DRN as compared to the control group. In addition, the grey value ratio was also higher in the FA group than in the SHAM group. (Control $n=4$, SHAM $n=6$ and FA $n=6$ per age) Data represent mean + SEM (ANOVA + Bonferroni; * $p<0.05 ;{ }^{* * *} p<0.001$ )

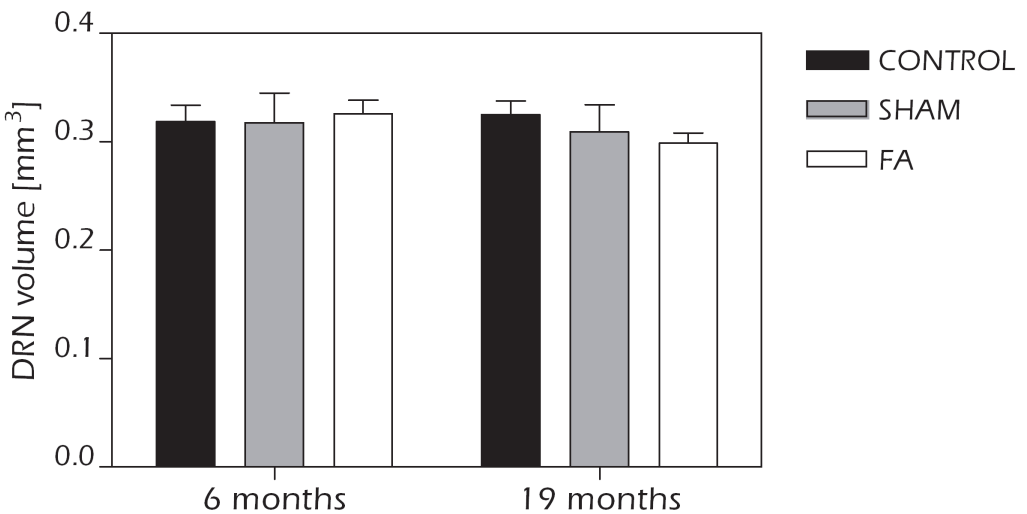

Figure 4: Fetal asphyxia did not affect the DRN volume.

Mean volume of the DRN at 6 and 19 months. At both time points, no significant alterations were found between groups. In addition, there was no effect of aging on the DRN volume. (Control $n=4$, SHAM $n=6$ and FA $n=6$ per age). Data represent mean + SEM. 


\section{Fetal asphyxia caused an age-related decrease in 5-HT- positive neuron number and density}

Total neuron numbers and mean neuron densities, as well as 5-HT-positive neuron numbers and densities are depicted in figure 5 A-D. At the age of 6 months, no significant differences were found between groups for any of the parameters measured. However, the mean density of serotonergic neurons (Bonferroni; $\mathrm{p}=0.041$; -9\%; figure 5D) was significantly lower in the FA group compared with the control group at 19 months. Additionally, multivariate analysis showed that aging significantly lowered the total number of neurons (figure 5A), total number of 5-HT-positive neurons (figure $5 \mathrm{C}$ ), mean neuron density (figure 5B) and the mean 5-HT-positive neuron density (figure 5D) (Multivariate ANOVA; respectively (resp.) $p=0.044 ; p<0.001 ; p=0.003 ; p=0.035$ ). This aging effect could be almost entirely ascribed to the FA group (Student t-test 6 vs 19 months; resp. $p=0.01 ; p=0.009 ; p=0.01 ; p=0.045$; resp. $-9 \%,-21 \%,-6 \%$, $-8 \%)$. Furthermore, aging significantly decreased the mean 5-HT neuron density and total 5-HT neuron number within the SHAM group as well /Student ttest; resp. $p=0.02 ; p=0.02$; resp. $-15 \%,-4 \%$ ). In contrast, aging did not affect any of the measured parameters within the control animals.

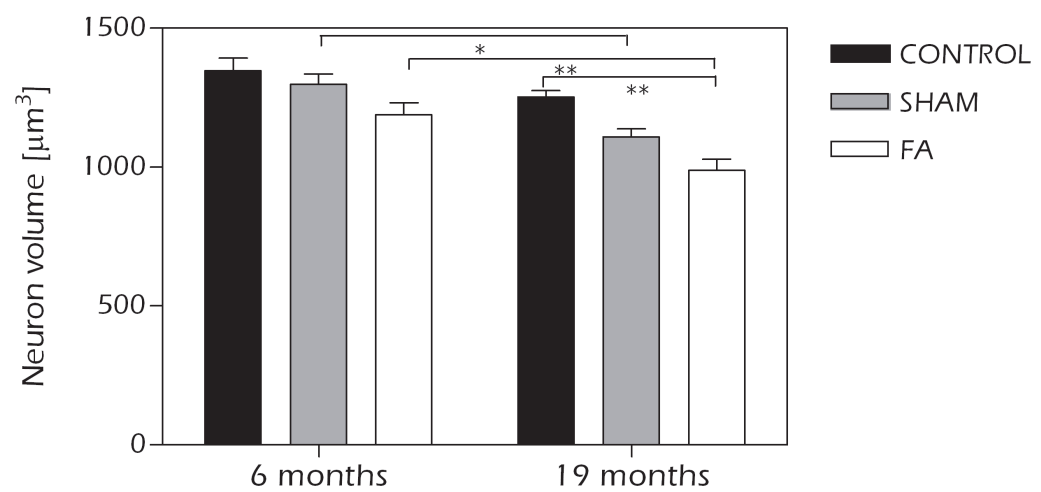

Figure 6: The effect of fetal asphyxia on cell size of serotonergic neurons in the DRN.

Mean volume of serotonergic neurons at 6 and 19 months. No significant changes were found between groups in 6-month-old rats. FA decreased the mean neuron volume as compared to the control group at the age of 19 months. Moreover, 19-month-old SHAM and FA rats had a significant lower neuron volume in comparison to their 6-month-old counterparts. (Control $n=4$, SHAM $\mathrm{n}=6$ and $\mathrm{FA} \mathrm{n}=6$ per age). Data represent mean + SEM. (ANOVA + Bonferroni; * $p<0.05 ;{ }^{* *} p<0.01$ ) 


\section{Fetal asphyxia lowered the mean volume of 5-HT- positive neurons}

As shown in figure 6, no significant effects on neuron volume were found at 6 months. In the 19-month-old animals, the mean neuron volume was significantly lower in the FA group compared to the control group (Bonferroni; $\mathrm{p}=0.002 ;-21 \%)$. Furthermore, mean neuron volume in both the SHAM (Student t-test; $p=0.04 ;-12 \%$ ) and FA group (Student t-test; $p=0.006 ;-17 \%$ ) declined significantly over time.
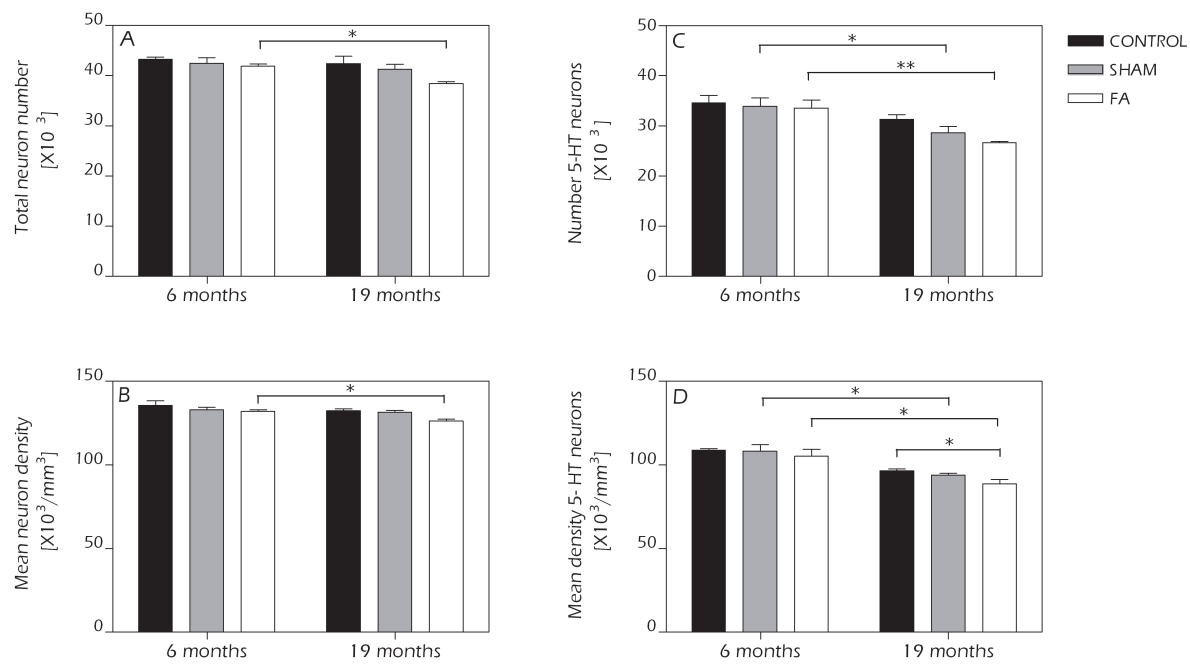

Figure 5: The effect of fetal asphyxia on neuron numbers and densities in the DRN

(A) Total number neurons $\left(\times 10^{3}\right)$ in the DRN at 6 and 19 months. No significant differences were observed at either 6 or 19 months. However, there was a significant effect of age within FA group. (B) Mean neuron density $\left(\times 10^{3}\right.$ cells $\left./ \mathrm{mm}^{3}\right)$ in the DRN. At either 6 or 19 months, no significant differences were found. There was a significant decrease within the FA group in the 19-month-old animals compared to the 6-month-old animals. (C) Total number of 5-HT positive neurons $\left(\times 10^{3}\right)$ in the DRN at 6 and 19 months. No significant changes were found at either 6 or 19 months. The 19-month-old FA and SHAM animals had significant less serotonergic neurons than their younger counterparts. (D) Mean density of 5-HT-positive neurons $\left(\times 10^{3} \mathrm{cells} / \mathrm{mm}^{3}\right)$ in the DRN at 6 and 19 months. At 6 months of age, no significant differences were observed. At 19 months, FA animals had a significant lower density of 5-HT-positive neurons than the control animals. In addition, there was a significant effect of age within both the SHAM and FA group. (Control $n=4$, SHAM $n=6$ and FA $n=6$ per age). Data represent mean + SEM. (ANOVA + Bonferroni; ${ }^{*} p<0.05 ;{ }^{* *} p<0.01$ ) 


\section{Discussion}

The present study examined the long-term effects of FA on anxiety-related behavior and the serotonergic circuitry of the DRN in adult and middle-aged rats. The data showed that FA increases anxiety-related behavior at 19 months of age. Our results demonstrated a decline in the mean density and the mean volume of DRN serotonergic neurons of 19-month-old FA rats compared to control animals as well as an age-related reduction in the total number, the mean density and the volume of serotonergic neurons in FA animals. Additionally, 19-month-old FA animals had a higher 5-HT immunoreactivity in the DRN.

\section{Induction of fetal asphyxia and serotonergic develop- ment at E17}

The complete 75 minutes of clamping both uterine and ovarian arteries, followed by reperfusion, induces FA by impairing the utero-placental circulation. Such a global event is associated with both fetal metabolic and hemodynamic changes, as well as with selective cerebral damage [237]. The fetal sheep is the most used and well-defined model of global fetal asphyxia. It is induced by umbilical cord occlusion [238]. The fetal sheep, however, is not a long-term survival model and does not allow behavioural testing. To solve this limitation, Cai et al used a similar rat model that induces asphyxia by clamping the uterine and ovarian circulation for 30 minutes. In this rat model NOS alterations and learning deficits were demonstrated at postnatal day 28 [93]. No previous work is available on the role of 5-HT in FA. However, the fetal period is very important for the ontogeny of serotonergic structures. Around E11-E 12 the first serotonergic cells are already born and from E 12 to E 18 they increase in number, acquire a more complex dendritic structure and migrate to their final positions[239-242]. We induced FA at E17, an important time point because serotonergic ascending and descending projections are still developing. 


\section{Fetal asphyxia causes an age-related decline in the total number, density and volume of serotonergic neurons in the DRN}

At 19 months, the DRN neuron volume as well as the number and density of serotonergic neurons were smaller in the FA group compared to the control group. Furthermore, there was a significant age-related decrease of the total number and mean density of DRN serotonergic neurons within the FA group. This neuron loss was associated with shrinkage of the remaining neurons. No such changes were found within the control group.

Traditionally, several animal and human studies described that aging can lead to a substantial loss of neurons throughout the brain [243]. For example, Henderson and collegues described a substantial loss in neuron density in the somatosensory cortex [244]. Likewise, Brizzee et al reported a clear age-related decline in hippocampal CA1 neurons [245]. Aging has also been linked to shrinkage of neurons. Smaller perikaryon and smaller nucleus were found in the human and rat hippocampus with increasing age [246, 247]. For that reason, a possible explanation for the age-related changes in our study could be that FA induces an acceleration of the aging process, by increasing the rate of normal age-related processes, like atrophy and neurodegeneration. Of note, rats are generally considered aged at approximately 24-28 months, which is the equivalent to humans more or less in their seventies. Therefore, rats used in our study were middle-aged, i.e., 19 months of age, which supports the theory of accelerated aging.

Recently, improved methods, like design-based stereology, indicated that neuronal death is not that prominent during normal aging [248]. Neuronal loss seems not as inevitable as previously was presumed. Furthermore, those new studies showed that the extent of neuronal loss can vary tremendously between different brain regions, with some brain regions being more vulnerable than others [249]. In our study, no age-related decline was found within the control group. Additionally, no loss or shrinkage of DRN neurons has been reported yet in normal aged humans or animals. For those reasons, it is possible that the observed changes in neuron number and volume are due to diseasemediated aging, rather than a normal aging process. Possibly, FA makes the DRN neurons more prone to neurodegeneration. 
It is interesting to note that a smaller neuronal size may be related to a decline in cellular function, like lower protein synthesis or a lower metabolic capacity [250]. Consequently, the decline in cell volume found in this study might implicate an impaired 5-HT neurotransmission. In addition, neuronal shrinkage always precedes neuronal cell death [251]. Therefore, the smaller neuronal size may reflect an early phase of neurodegeneration, contributing to the loss of serotonergic neurons. Neuronal shrinkage and neurodegeneration are also accompanied by the loss of dendrites and axons, resulting in a diminished number of synapses. This may result in functional impairments, which can have important behavioral consequences. A last explanation for the smaller neurons might be the selective loss of the largest neurons. In age-related diseases like Alzheimer's disease, it has been shown that the decrease in mean neuronal volume is often due to loss of larger neurons and preservation of the smaller ones [252].

We are the first to propose the concept of accelerated aging in relation to asphyxia during the fetal period. The link between term perinatal asphyxia and an accelerated vulnerability of dopaminergic neurons was already suggested by Loidl et al. [166]. Similarly, Weitzdoerfer et al. postulated that age-related behavioral changes, like aggressive behavior and decreased social exploration, are intensified after perinatal asphyxia [103]. Additionally, it is interesting to note that Nyakas et al. found anatomical abnormalities in serotonergic fibers of 28-month-old rats after perinatal nitrite exposure [253].

Although the 5-HT neuron density and number were decreased, the 5HT-immunoreactivity was increased in the FA animals at the age of 19 months. There are a few possible explanations that may clarify this contrasting finding. The first explanation could be that the enhanced IR may represent an increased 5HT content inside the cell [254]. The loss of 5-HT neurons might elicit a compensatory mechanism trying to maintain normal 5-HT levels in the DRN. An increase in intracellular 5-HT content can be caused by different mechanisms, like an enhanced synthesis, a decreased degeneration or a changed reuptake capacity of 5-HT. An increased reuptake was already demonstrated by Weitzdoerfer and collegues [102]. They showed increased serotonin transporter (5$\mathrm{HTT}$ ) levels in the hippocampus of 2-year-old rats exposed to perinatal asphyxia. It might be that a similar mechanism is triggered in the DRN after FA. Another 
possible explanation could be that the higher 5-HT-IR represents only relative increase because of smaller neuronal volumes. Smaller volume together with normal or elevated 5-HT levels might cause a relative higher than normal 5-HT concentration inside the cell.

Since the sham-operation, without FA procedure, altered also the serotonin biochemistry, we suggest that the sham-operation also causes a mild form of fetal asphyxia. It seems plausible that the interventions (i.e. laparotomy and extra-abdominal exposure of the uterine horns for 75 minutes) might cause changes in uterine blood flow, thereby affecting the oxygenation to the fetuses. Although the literature is very contradictory, part of the changes may also be caused by the anesthetics (e.g. combination of xyalzine and ketamine). According to Young et al. and Rudin et al., a single exposure to ketamine can induce apoptotic neurodegeneration in different regions of the developing brain $[198,199]$. Currently, there is no literature available on the effects of $x y-$ lazine and ketamine on the 5-HT neurons in the DRN. Furthermore, it is important to note that the results of both groups (SHAM and FA) are not completely similar. The neuron loss was graded, being more severe in the FA group than in the SHAM group.

\section{Fetal asphyxia increased anxiety-related behavior at 19 months}

In the present study, FA rats spent more time in the corners and less time along the walls of the open field at 19 months of age. These observations point towards higher levels of anxiety in comparison to control animals. These results are in line with a previous report of Weitzdoerfer and collegues [103], who showed an increase in anxiety-related behavior in 2-year-old rats exposed to perinatal asphyxia.

The increased anxiety-related behavior following FA can be explained by the alterations found in the serotonergic system of the DRN. 5-HT has been implicated in the neurochemistry of anxiety for a long time. However, there is no agreement on whether anxiety results from excessive or deficient 5-HT functioning. This discrepancy can be explained by the current thought that a com- 
bination of specific functional and/or anatomical pathways and different 5-HT receptor subtypes may be responsible for distinct types of anxiety [231, 255].

It is important to notice that anxiety is one of the emotional behavioral changes associated with aging. Boguszewski and colleagues observed significantly increased anxiety levels in 24-month-old rats compared to their 4-month-old counterparts [256]. Therefore, these behavioral changes may also support our idea that the aging process is intensified by an asphyxic insult during fetal brain development. This idea awaits further research though.

\section{Fetal asphyxia: a link with affective disorders?}

It is widely accepted that a deficient $5-\mathrm{HT}$ transmission contributes to various neuropsychiatric behavioral changes, with depressive and anxiety disorders being the most important ones [228]. The underlying causes of these disorders are unknown. The etiology is probably multifactorial, with genetic as well as psychosocial factors playing an essential role. These causations, however, can only clarify a small part of the disease. Nevertheless, it is known that 5-HT plays an important role in normal brain development, before playing a role as a neurotransmitter in the mature brain [232]. Fetal insults could possibly cause disruptions in serotonergic development, thereby contributing to the development of affective disorders. Furthermore, it is important to note that very low birth weight is associated with anxiety-related disorders in adolescents [257]. In addition, it has been suggested that age-related serotonergic changes are associated with both late-life cognitive and mood disorders, like geriatric depression [258]. It seems plausible that FA could accelerate the onset and the progression of age-related loss of serotonergic neurons, enhancing the vulnerability to develop mood disorders later in life. Therefore these data might have important implications for mood-related research. All the assumptions made above await further research though.

\section{General conclusion}

This is the first study reporting increased anxiety-related behavior and age-related changes in 5-HT immunohistochemistry within the DRN in fetal asphyxic 
rats. These age-related changes suggest that $F A$ accelerates the aging process. Furthermore, the data imply that FA might be associated with the development of affective disorders. 

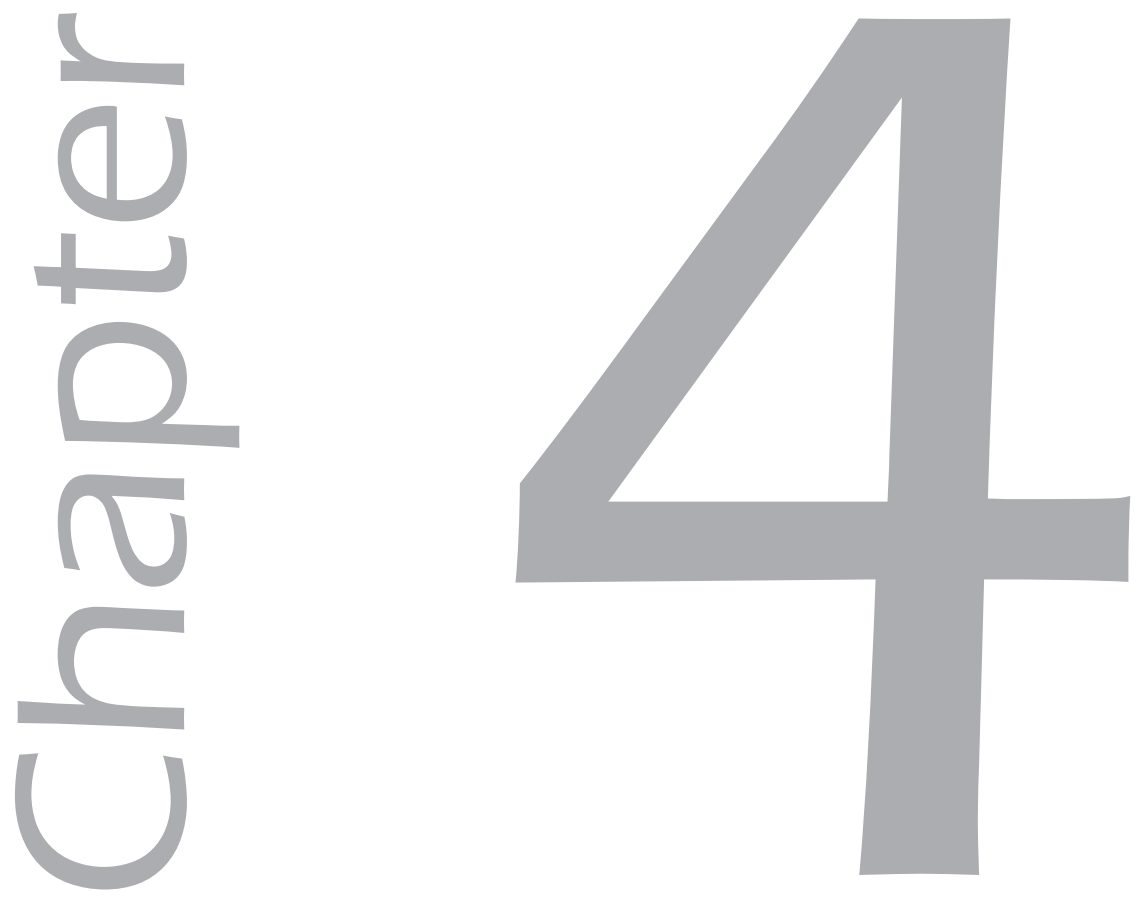

Fetal asphyxia leads to the loss of striatal presynaptic boutons in adult rats

E.Strackx, D.L.A. Van den Hove, H.P. Steinbusch, H.W.M. Steinbusch, J.S.H. Vles, C.E. Blanco and A.W.D. Gavilanes

International Journal of Developmental Neuroscience (2009); In press 


\begin{abstract}
Fetal asphyxic insults in the brain are known to be associated with developmental and neurological problems like neuromotor disorders and cognitive deficits. Little is known, however, about the long-term consequences of fetal asphyxia contributing to the development of different neurological diseases common in the adult or the aging brain. For that reason the present study aimed to investigate the long-term effects of fetal asphyxia on synaptic organization within the adult rat brain. Fetal asphyxia was induced at embryonic day 17 by 75-minute clamping of the uterine and ovarian arteries. Presynaptic bouton densities and numbers were analyzed in the striatum and prefrontal cortex at the age of 19 months. A substantial decrease in presynaptic bouton density and number was observed in the striatum of fetal asphyxia rats compared to control rats, while an increase was found in the fifth layer of the prefrontal cortex. These results suggest that fetal asphyxia can have long-lasting effects on synaptic organization that might contribute to a developmental etiology of different neurological disorders and aging.
\end{abstract}




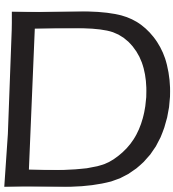

evelopmental asphyxia is an important cause of neonatal mortality and morbidity, frequently associated with long-term motor, behavioral and cognitive deficits in survivors [6, 259, 260]. Nowadays, numerous reports are available studying the different short-term clinical and morphological consequences of fetal asphyxia [261]. In contrast, one might assume that there may be also long-term effects of fetal asphyxia, for example during adolescence or even in adulthood. We have recently shown that fetal asphyxia has long-lasting effects on both dopamine-mediated motor and serotonin-mediated anxiety behavior and biochemistry. Fetal asphyxia leads to a decrease in basal and amphetamine-induced locomotion in 6-month-old rats, which was related to the loss of dopaminergic neurons in the substantia nigra pars compacta (SNC) [153]. Additionally, fetal asphyxia causes increased anxiety-related behavior associated with the loss of serotonergic neurons in the dorsal raphe nucleus (DRN) at the age of 19 months [152]. Looking for more evidence of long-lasting effects of fetal asphyxia on the aging brain, this study aimed to determine the effect on synapse numbers at the age of 19 month in two different brain areas, i.e., the striatum and the prefrontal cortex (PFC). These aims are chosen, because: 1) the striatum and the PFC are associated with locomotor and anxiety-related behavior respectively, two types of behavior which are known to be affected after fetal asphyxia [153, 262], 2) both structures have been shown to be vulnerable to hypoxic-ischemic damage $[57,59]$ and 3) the number of functional connections between cells is an important parameter that contributes to neuronal functioning.

\section{Materials and methods}

\section{Animals}

All experiments were approved by Animal Ethics Board of Maastricht University on animal welfare according to Dutch governmental regulations. All efforts were made to minimize the pain and stress levels experienced by the animals and to minimize the number of rats necessary to produce reliable scientific data. Inbred date-pregnant Lewis rats were supplied on day 14 of ges- 
tation (E 14) by the Animal Care Department of Maastricht University and were housed individually at its facilities. The rats were kept under standard laboratory conditions with food and water given ad libitum, $21 \pm 2^{\circ} \mathrm{C}$ environment temperature, a 12h light/dark schedule (lights on at 07:00 h) and background noise provided by radio. Exclusively male offspring were used within this study, because both morphological and behavioral evidence show a differential vulnerability to a birth insult in males versus females. A greater impact is seen in the male gender, probably due to the protecting role of the circulating estrogens in females [191, 192].

\section{Fetal asphyxia procedure}

At E17 fetuses were exposed to severe fetal asphyxia as previously described [153]. In brief, pregnant rats were anaesthetized by xylazine ( $1 \mathrm{mg} / \mathrm{kg}$ s.c.) and ketamine (50mg/kg i.m.). Rats were subjected to a midline laparotomy to expose both uterine horns. Fetal asphyxia was achieved by completely clamping both uterine and ovarian arteries with removable clamps. In the mean time, the uterine horns were kept wet, by rinsing them with saline $0.9 \%$. Additionally, dams were injected subcutaneous with saline $0.9 \% 15 \mathrm{ml} / 100 \mathrm{~g}$ interscapularly) to prevent dehydration. After 75 minutes, the clamps were removed to allow reperfusion. The uterine horns were placed back intra-abdominally and the abdominal wall was repaired. Sham-operated rats underwent the same procedure except for clamping the arteries. The fetal asphyxic, sham-operated and control groups were allowed to deliver normally (i.e. full-term vaginally delivered on E21-22). All procedures were performed within a controlled environment $\left(37^{\circ} \mathrm{C}, 80 \%\right.$ humidity, room air). No more than two pups per litter were used to prevent litter effects. They were randomly cross-fostered to surrogate dams, which had given birth normally earlier that day.

\section{Perfusion and tissue preparation}

At 19 months of age, fetal asphyctic $(n=6)$, sham $(n=6)$ and control $(n=4)$ rats were deeply anaesthetized with sodium pentobarbital $160 \mathrm{mg} / \mathrm{kg}$ i.p.; Nembutal ${ }^{\circledR} \mid$ and transcardially perfused with ice-cold 4\% paraformaldehyde in $0.1 \mathrm{M}$ phosphate buffer ( $\mathrm{pH}$ 7.6). The brains were dissected rapidly from the skull and 
post-fixed for $2 \mathrm{~h}$ at $4^{\circ} \mathrm{C}$ in the same fixative. The brains were suspended in 15\% sucrose for $48 \mathrm{~h}$. Brains were cut serially at $-30^{\circ} \mathrm{C}$ into $30 \mu \mathrm{m}$ thick coronal cryostate (Leica CM 350, Germany) sections at the following bregmas: 1.60mm to $-0.80 \mathrm{~mm}$.

\section{Synaptophysin immunohistochemistry}

Synaptophysin immunostaining was used to analyze presynaptic bouton densities. Synaptophysin is an integral membrane protein located in the synaptic vesicles and therefore a good marker to detect nerve terminals. All immunohistochemical reactions were carried out in a free-floating manner. They were processed simultaneously to guarantee identical conditions. All washing and dilutions steps of the antibodies were done by TBS (0,01M) with 0,2\% Triton$\mathrm{X}-100$ (TBS-T) at room temperature. In order to minimize the background staining, all sections were pre-incubated with 5\% normal goat serum (Sigma, The Netherlands) for 30 minutes. Furthermore, normal goat serum (2,5\%) was added to all solutions containing antibodies. An anti-synaptophysin antibody (monoclonal mouse; Boehringer-Mannheim, Germany) was used as a primary antibody overnight at a dilution of $1: 35$ at $4^{\circ} \mathrm{C}$, followed by immersion with a goat anti-mouse biotinylated secondary antibody 11:150; American Qualex, CA) for $1 \mathrm{~h}$. Brain sections were further processed using the avidin biotin complex technique with 3,3-diaminobenzidine (DAB) to obtain a color reaction (Vector laboratories, USA). After the labeling procedures, sections were mounted on gelatine-coated glass slides, air-dried and dehydrated in ascending ethanol concentrations, cleared with xylene and cover-slipped with DePex.

\section{Stereological analysis: volume estimates}

For stereological analysis, a Nissl staining was used. Sections were dried, defatted with a Triton-X100 solution and stained with cresyl violet for the detection of Nissl body in the cytoplasm of neurons $(0.01 \%, 11 \mathrm{~min})$. Slides were coverslipped with DePeX.

Design-based stereology was performed for volume measurements using a stereological computer microscopy system and Stereolnvestigator software (Mi- 
croBrightField, Williston, VT, USA). The regions of interest (striatum and PFC) were delineated according to Paxinos and Watson (1986) by anatomical landmarks with a $1.25 \mathrm{x}$ magnification on live microscopic video images displayed on a monitor. Volumes were estimated by multiplying the average surface area with the measured average section thickness (after histological processing), the number of sections investigated, and a factor of eight since every eighth section was used [193].

\section{Quantitative analysis of synaptophysin staining}

Presynaptic bouton densities were analyzed in the frontal cortex and striatum. Delineation of the different areas occurred as described by Van de Berg et al. according to anatomical boundaries [57]. In the PFC measurements were performed for each of the six cortical laminae, in two adjacent areas of $5000 \mu \mathrm{m}^{2}$, while the striatum was separated in four distinct regions: dorsal, ventral, medial and lateral. Measurements were performed in those 4 areas in two adjacent areas of $5000 \mu \mathrm{m}^{2}$ and the data of all 4 regions were pooled.

The immunoreactive punctae were estimated by calculating the density of the synaptophysin-immunoreactive presynaptic boutons, like previously described by Van de Berg et al [57]. Analysis was done using an Olympus AX-70 microscope. For each subregion, photos were taken at magnification $\times 100$, using a Olympus F-view cooled CCD camera (Olympus, Tokyo). Three different sections of each animal (interaural $10.20 \mathrm{~mm}, 9.48 \mathrm{~mm}$ and $7.84 \mathrm{~mm}$ ) were analyzed in the above described areas, yielding $36 / 6$ layers $x 2$ adjacent areas $x 3$ sections) photos for the PFC and 24 (4 subregions $x 2$ adjacent areas $x 3$ sections) photos for the striatum of each animal.

The immunoreactive punctae were detected, using the image analyzing system CellP (Soft Imaging System, Münster, Germany). All measurements were performed at a single focal plane. Background levels were equalized and a shading correction was carried out by the software to correct for irregularities in illumination. Figure 1B gives an example of an edited photograph. Using a trial and error method, the threshold values, providing the most accurate measurement compared to direct visual counting, were selected. Once the ideal threshold value was found, it was saved in the computer program and kept 
the same for all measurements. All blood vessels and cell bodies and cortical tissue out of focus were excluded (Figure 1A).

\section{Calculation of the presynsptic bouton numbers}

Presynaptic bouton numbers were calculated by multiplying the individual density data with the corresponding volume data, not taking into account the dimensions of the investigated parameters (i.e., $1 / \mu \mathrm{m}^{2}$ for density and $\mathrm{mm}^{3}$ for volume). Although these numbers are not unbiased because the densities were analyzed in two dimensions (= one focal plane) instead of three dimensions, they give a reliable estimation $[263,264]$. It is not possible to calculate the exact total number of synapses, since size, shape and geometric orientation of the presynaptic boutons are unknown

\section{Statistical analysis}

All date are expressed as mean + standard error of the mean (SEM). Comparisons were tested with an one way analysis of variance (ANOVA) in the striatum and the different layers of the PFC. Significant effects were further analyzed by post-hoc Bonferroni corrections. The level of significance was set at $p<0.05$. All calculations were performed using standard statistical software (SPSS 12.0 software).

\section{Results}

Figures $1 \mathrm{~A}$ and $\mathrm{B}$ illustrate an example of a synaptophysin staining in the striatum of a control animal. The synaptophysin immunoreactivity was restricted to small punctae, representing presynaptic boutons. There were no apparent differences in the morphological appearance of the punctae between groups.

Figures $2 A$ and $B$ show the results of the presynaptic bouton densities in the striatum (Figure 2A) and the different layers of the PFC (Figure 2B). In the striatum, one-way ANOVA analysis showed an overall effect of group on mean 
presynaptic bouton density $(p<0.05)$. Fetal asphyctic animals had a lower bouton density than control animals $(-38 \% ; p<0.05)$, as indicated by Bonferroni post-hoc testing. Sham animals did not differ from either fetal asphyctic or control animals. In the PFC, one-way ANOVA analysis showed an overall difference between groups in mean presynaptic bouton density in the 5th layer $(p<0.05)$. Nevertheless post-hoc testing revealed no significant differences between groups.
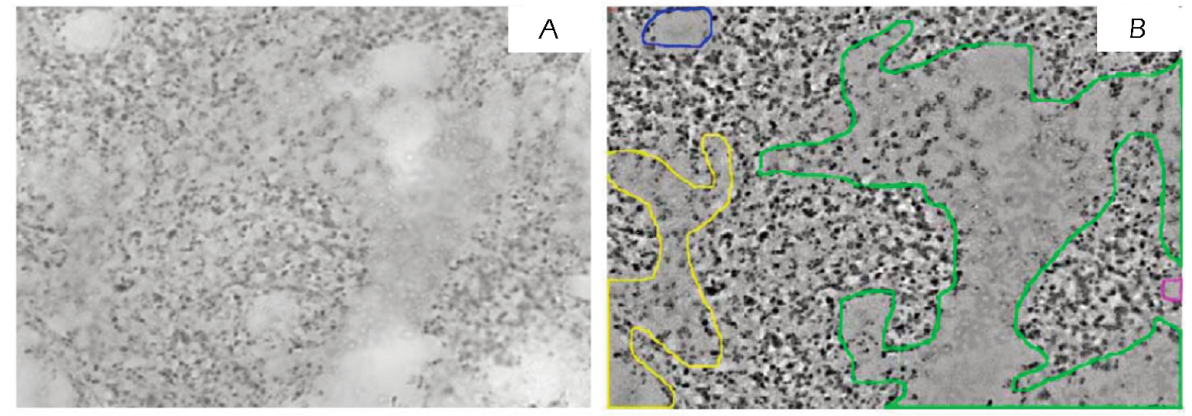

Figure 1: Example of an edited and unedited photograph of a synaptophysin-stained section.

(A) Unedited photograph of a synaptophysin stained section obtained from the striatum of a control animal. (B) Edited photograph, showing the modified punctae, as acquired from the image analysis system. Areas out of focus, artifact, blood vessels and cell bodies were excluded (delineated areas).

Figures $2 \mathrm{C}$ and $\mathrm{D}$ depict the results of presynaptic bouton numbers in the striatum (Figure 2C) and the layers of the PFC (Figure 2D). In the striatum, an overall difference was found by one-way ANOVA analysis $(p<0.05)$. Post-hoc Bonferroni corrections showed that fetal asphyctic animals had a significant smaller number of boutons compared to control animals (-42\%; $p<0.05)$. Again the sham group did not differ from the control and fetal asphyxia group. In the PFC, one-way ANOVA analysis demonstrated significant differences in presynaptic bouton numbers in the 5 th layer (respectively $\mathrm{p}<0.05$ ). Higher bouton numbers were found in all layers of fetal asphyctic animals. Post-hoc Bonferroni testing showed that fetal asphyctic animals had significant more presynaptic boutons in the 5 th layer $(+34 \%$; $p<0.01)$ than control animals.

For none of the investigated brain areas significant differences in volume were found between groups (Figures 2B and E). 
Overall, these data show a decrease in the number and density of presynaptic boutons in the striatum and an increase in the number of presynaptic boutons in the 5th layer of PFC.
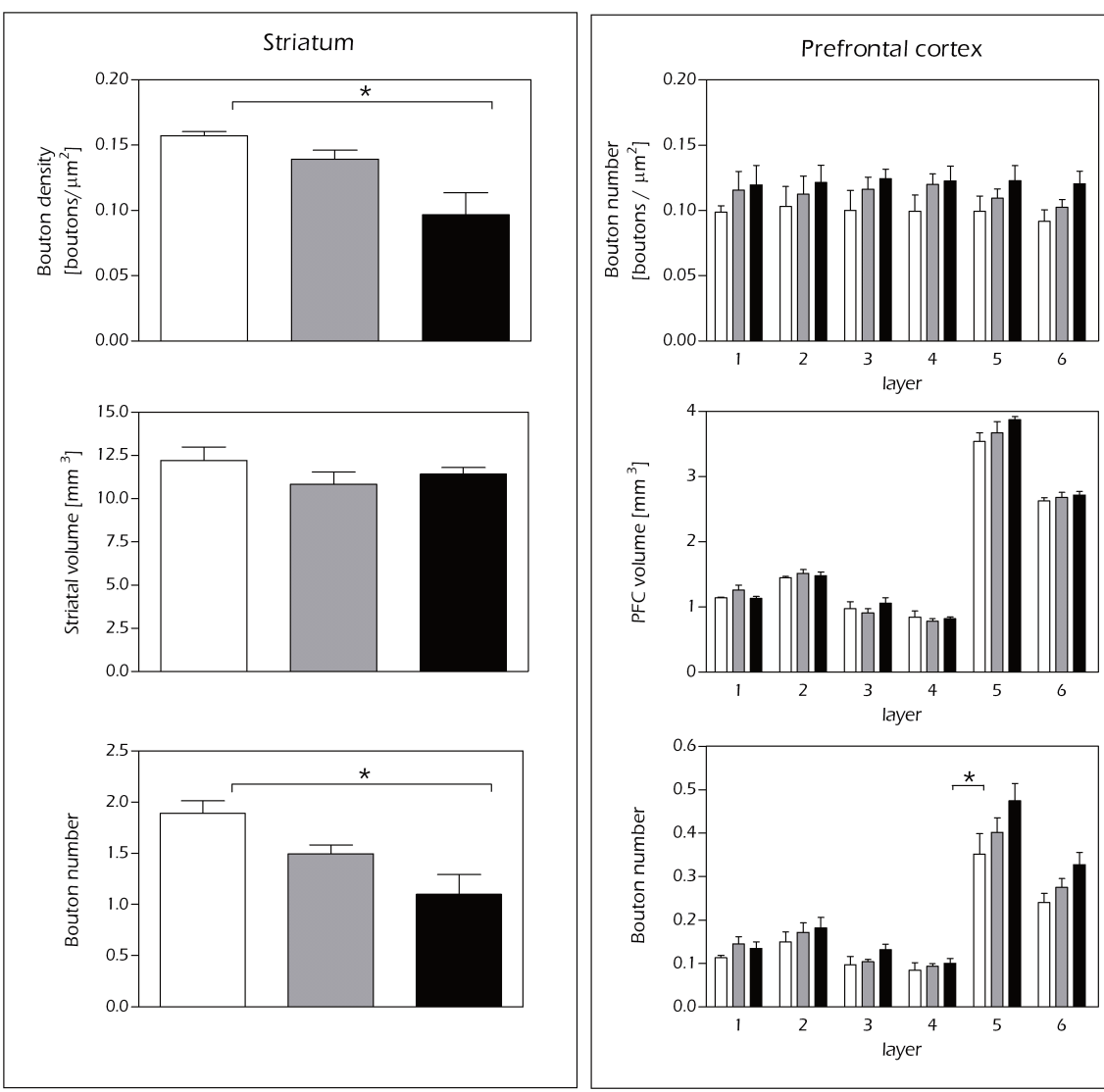

$$
\varpi \text { CONTROL }
$$

Figure 2: Results of the quantitative analysis of presynaptic boutons in the striatum and PFC

(A) Mean density of presynaptic boutons in the striatum. Fetal asphyctic animals showed a significant lower bouton density compared to control animals. (B) Mean striatal volume. No significant differences were seen between the groups. (C) Estimated number of presynaptic boutons in striatum. Fetal asphyctic animals showed a decreased number of boutons in comparison to control animals (D) Mean density of presynaptic boutons in the different layers of the PFC. No significant differences were found between groups. (E) Mean volume of the different layers of the PFC. No significant differences were seen between the groups. (F) Estimated number of presynaptic boutons in the different layers of the PFC. There was a significant increase in the number of presynaptic boutons in layer 5 in the fetal asphyxia group compared to the control group. All data are expressed as mean + SEM. $\left({ }^{*} p<0.05\right)$ 


\section{Discussion}

The present study shows that a severe asphyctic insult during pregnancy, a critical period for brain development, can have long-lasting effects on synaptic densities and numbers. Nineteen-month-old fetal asphyctic rats showed a significant decrease in both the density and number of presynaptic boutons within the striatum as compared to control rats, whereas an increase in presynaptic bouton number was found in the fifth layer of the PFC.

Long-lasting synapse loss within the striatum, as seen in our rats at 19 months of age may be the result of synapse loss that occurs within the first minutes and hours after the hypoxic-ischemic insult. Several studies demonstrated a decline in both pre-and postsynaptic density in different areas of the brain immediately after the insult. For example, Horner et al. showed that transient global perinatal ischemia caused a marked decrease in postsynaptic density in the chick hippocampus [265]. Likewise, Stepanov et al. demonstrated a similar decrease in presynaptic bouton numbers in the cerebellar cortex of 1-day-old rat pups after fetal hypoxia [266]. Studies examining long-term effects of neonatal hypoxia and/or ischemia showed that synapse loss still exists a couple of weeks after the exposure. Accordingly, Ramirez et al. detected altered phospholipid patterns 30 days after hypoxia-ischemia, pointing to reduced hippocampal synaptogenesis [267]. An alternative explanation for the striatal synapse loss observed in our 19-month-old rats is that it is induced later in life. Normally, aging leads to synapse loss in a number of brain regions, like the striatum, parietal cortex and hippocampus. For example, Wong et al. reported a reduction in presynaptic bouton density in the parietal cortex of older rats compared to their younger counterparts [268]. Smith et al. also reported an age-related synapse loss is in the rat hippocampus [269] Of note, rats are generally considered aged at approximately 24-28 months, which is the equivalent to humans around their seventies. Therefore, rats used in our study were only middle-aged, i.e., 19 months of age. For that reason, the loss of striatal synapses is probably due to disease-mediated aging, rather than a normal aging process. In other words, fetal asphyxia might possibly induce a diseasemediated or accelerated aging process by increasing the rate of normal age- 
related processes like synapse loss or by making the brain more susceptible to neurodegeneration.

At a cellular level, the loss of striatal synapses can be due to several interacting factors. First, synapse loss might be caused by the loss of striatal neurons. Recent animal studies have indicated that the striatum is very vulnerable to fetal or neonatal hypoxia-ischemia. Striatal neuron loss in relation to developmental hypoxia-ischemia has been demonstrated previously. Both complete and partial umbilical cord occlusions in sheep resulted in the loss of striatal neurons, especially projection neurons and GABA-ergic interneurons [270-273]. Severe perinatal asphyxia in rats caused the loss of striatal projection and interneurons as well $[58,274]$. Second, striatal synapse loss could be caused by neuron loss in regions that have extensive projections to the striatum. One important structure that has projections to the striatum is the DRN. Approximately a third of all serotonergic DRN neurons project to the striatum [234].

Besides the DRN, also the SNC has extensive projections to the striatum [275]. Previous studies by our group already demonstrated that fetal asphyxia induces the loss of serotonergic neurons in the DRN in the same 19-month-old animals as well as to a loss of nigral dopaminergic neurons in 6-month-old animals [153, 262]. Therefore, the loss of striatal synapses might be partly explained by the loss of raphe serotonergic and nigral dopaminergic neurons. Third, neuronal changes can also lead to synapse loss. For example, degeneration of dendritic structures or retraction of axonal processes intruding on striatal neurons might cause synapse loss, without losing the neurons itself. An important remark to be made is that the loss of synapses often precedes the loss of neurons [276]. Which of these explanations might hold true awaits further research though.

Embryonic day 17 is an important time point in the development of both areas investigated in this study. The first structure is the striatum. According to the prediction model of Finlay and Darlington, striatal neurogenesis in rats peaks between post-conception days 14.4 and 17.3 [145]. This corresponds to the human equivalent, the caudatoputamen complex, which peaks at post-conception day 56, i.e. early gestation. The other structure of interest was the cortex. According to the same model, neurogenesis of the cortex, especially layer $\checkmark$, peaks between post-conception days 15.1 and 17.6. This corresponds to the 
development of the human cortex at post-conception day 79.6. In conclusion, $\mathrm{E} 17$ is an important moment during rat brain development since both the striatum and cortex peak neurogenesis around this time point.

Another important finding of this study is the regional difference in presynaptic bouton numbers. In contrast to a decreased number of presynaptic boutons in the striatum, the fifth layer of the PFC showed an increased number of presynaptic boutons. These findings support the hypothesis that brain trauma, like hypoxic-ischemic brain damage, could not only lead to synapse loss but also to the formation of new synaptic contacts. Accordingly, Stroemer et al. have shown an increase in synapses in the cortex following cerebral ischemia in the adult brain [277]. Van de Berg et al. revealed a similar increase in the parietal cortex of an immature brain after severe perinatal asphyxia [57]. They did, however, not find differences in the prefrontal cortex. This difference may be explained by the different timing of the insult. It could be that fetal asphyxia affects different brain areas than perinatal asphyxia, which is induced a few days later.

Smaller pyramidal neurons, located in upper infragranular layer $V$ of the frontal and PFC, project to the striatum [278-280]. Therefore, the increased number of synapses in the PFC may be an attempt to compensate for the loss of striatal synapses or neurons by allowing more neuronal communication. Similar compensatory mechanisms between two different areas have already been demonstrated in neurodegenerative diseases, like Alzheimer's disease [281]. Additionally, the synaptic density in the PFC is regulated by dopamine [282]. Dopaminergic fibers are mainly located in layer $\mathrm{V}$. The serotonergic neuron system, which originates in the DRN, inhibits dopaminergic function in the midbrain as well as the forebrain. As previously stated, the nigrostriatal dopaminergic as well the DRN serotonergic systems are affected in FA rats [262]. Therefore we hypothesized that the increased PFC synaptic density might result from a shift of the dopaminergic / serotonergic balance in favor of a disinhibited dopaminergic system, while it stays inhibited in control animals. This might lead to a higher than normal extracellular dopamine concentration in the PFC, which in turn leads to an increase in synaptic density.

An important remark to be made is that the sham-operation, without fetal asphyxia procedure, also altered the density and estimated number of synapses, although not significant. Therefore, we suggest that the sham-operation also 
causes a mild form of fetal asphyxia. It seems plausible that the interventions (i.e. laparotomy and extra-abdominal exposure of the uterine horns for 75 minutes) might cause changes in uterine blood flow, thereby affecting the oxygenation to the fetuses. It is important to note that the results of both groups (sham and fetal asphyxia) are not completely similar. The neuron loss was graded, being more severe in the fetal asphyxia group than in the sham group. Although the literature is very contradictory, part of the changes may also be caused by the anesthetic agents (e.g. xyalzine and ketamine). A single exposure to ketamine can induce apoptotic neurodegeneration in different brain regions of the 7-days old mouse [198, 199]. In contrary, other experimental studies showed that the use of the combination of ketamine and xylazine in pregnant rats at E12 does not influence cerebellar neuronal precursors at birth [283]. Additionally, a recent study even showed that the combination of ketamine and xylazine can induce nigrostriatal neuroprotection in two adult rat models for Parkinson's disease [200]. In conclusion, the data on the effects of ketamine plus xylazine are inconsistent, probably because the effects are highly depended on the maturational brain status. Therefore, further research is necessary to reveal the possible effect of these anesthetic agents on the fetal brain.

The findings of this study may be important in understanding the pathological mechanisms of fetal asphyxia. Although direct implications for the human fetus cannot be correctly extrapolated yet, this study confirms again that fetal asphyxia can cause subtle but long-lasting changes in the brain. These changes may be relevant for better understanding the pathogenesis of different neurological disorders. Additionally, the results of this study might explain some of the behavioral changes due to fetal asphyxia. Motor impairments have been demonstrated in these animals before. At the age of 6 months, fetal asphyctic rats showed a reduction in basal as well as amphetamine-induced locomotion during open field testing [153]. Since the striatum plays a crucial role in motor control, alterations in striatal synaptic numbers might be associated with these deficits in motor function. Moreover, that same study demonstrated that these impairments in motor function were associated with the loss of dopaminergic in the SNC. This correlates with our current data, since the SNC has extensive projections to the striatum. For that reason, the loss of nigral neurons might cause the loss of striatal synapses. 
In conclusion, the present study is the first to demonstrate that fetal asphyxia has long-lasting effects on synaptic numbers. These changes were region-specific, showing synapse loss in the striatum and an increase in synapse numbers in the 5th layer of the PFC. Additionally, these data may contribute to the hypothesis that fetal asphyxia can cause subtle, but long-lasting changes that may be relevant to the pathophysiology of different neurological disorders. 

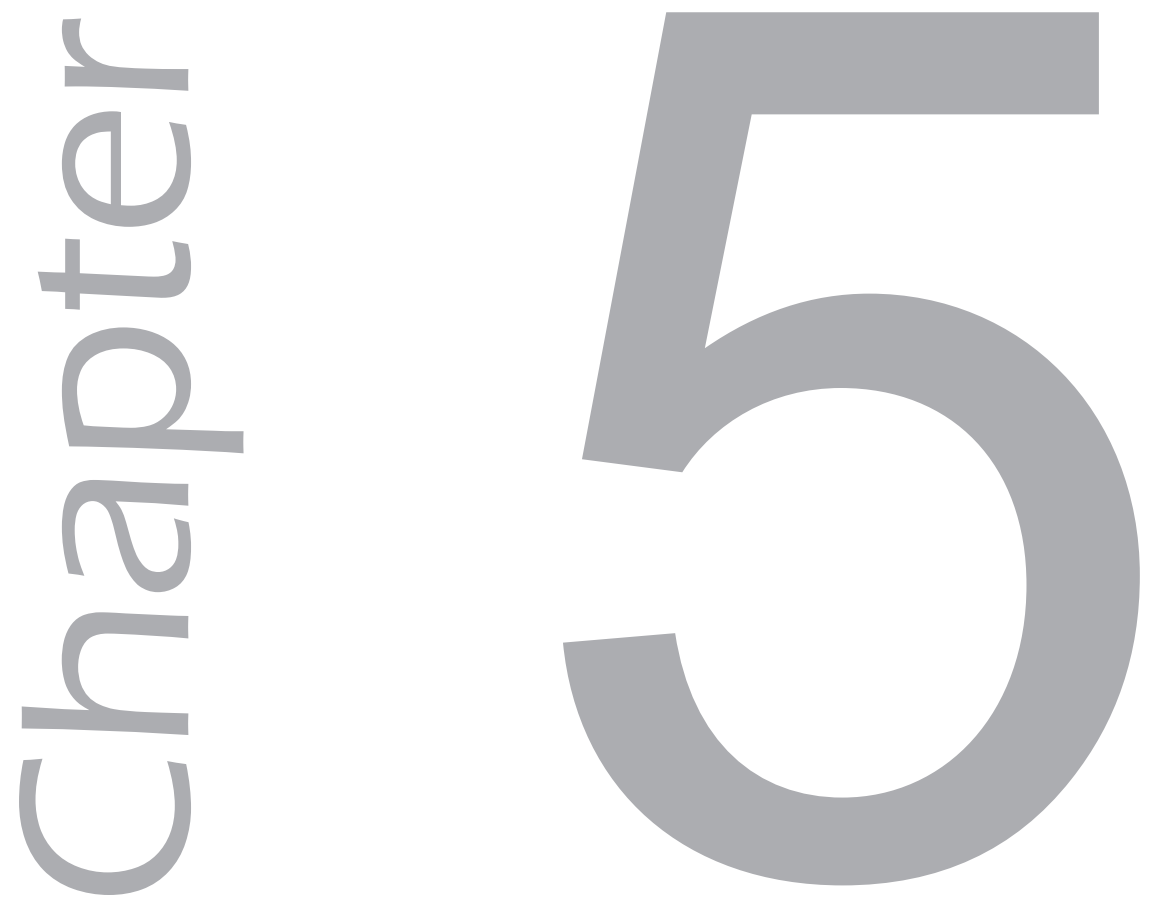

\section{Alternations of brain apoptotic cell death and carotid artery reactivity in a fetal asphyxia preconditioning model}

E.Strackx, B. Zoer, D.L.A. Van den Hove, H.P. Steinbusch, H.W.M. Steinbusch, C.E. Blanco, J.S.H. Vles, E. Villamor and A.W.D. Gavilanes 


\section{Abstract}

Perinatal asphyxia is one of the major causes of impaired development in infants. Interestingly, brief episodes of experimental hypoxia-ischemia (HI) can provoke an endogenous neuroprotection against subsequent more severe insults. Little is known about the exact mechanisms underlying this phenomenon called "HI preconditioning". The aim of this work was to develop a new model of fetal HI preconditioning by combining two global asphyctic insults. These global insults reflect the pathophysiological conditions of asphyxia before and during birth more closely than the existing neonatal stroke models. A fetal asphyctic insult (FA) was induced, as a preconditioning stimulus, by clamping the uterine vasculature for 30 minutes on embryonic day 17 (E 17, first hit). At birth (PO), severe perinatal asphyxia (SPA) was induced by placing the uterine horns, still containing the fetuses, in a water bath for 19 minutes. At P4, carotid arteries were studied in a wire myograph and at P8 brains were analyzed for apoptotic cell death in the prefrontal cortex and striatum, using a terminal deoxynucleotidyl transferase-mediated dUTP-biotin nick end-labeling (TUNEL) staining. Results show that animals that underwent FA (FA and $\mathrm{FA}+\mathrm{SPA}$ ) had significantly lower birth weights than SPA and control animals (7.3\%; $p=0.044)$, while at P8 SPA animals were significantly lighter. At P4, the contraction induced by $\mathrm{K}+$ was significantly reduced in the carotid arteries from the SPA group and endothelium-dependent relaxation (mediated by acetylcholine) was augmented in the FA group. These changes in vascular responsiveness were not present in the animals exposed to both insults (FA + SPA). Additionally, FA+SPA animals showed lower numbers of apoptotic cells compared to SPA animals in both the prefrontal cortex $(-44 \% ; p=0.003)$ and striatum (-46.4\%; $p<0.001$ ) at P8. We can conclude that exposure to a global fetal asphyctic insult seems to protect against the vascular alterations and the increase of apoptotic cell death in striatum and prefrontal cortex induced by a severe perinatal asphyctic insult at birth. Therefore, this model offers new possibilities to study the effects of global fetal HI preconditioning on post-asphyxtic encephalopathy at birth. 


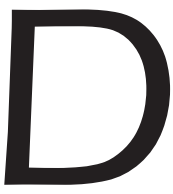

evelopmental asphyxia is a major cause of neonatal mortality and morbidity, which is frequently associated with long-term motor, behavioral and cognitive deficits in survivors [6, 161, 259-261, 284]. Although our understanding has increased considerably during the last decade, there is still a lack of effective therapeutic strategies against asphyctic brain damage. Interestingly, brief episodes of hypoxia-ischemia (HI) in the brain can provoke an endogenous neuroprotection against subsequent more severe episodes. This phenomenon is called "hypoxic-ischemic preconditioning" or "hypoxic-ischemic tolerance". Little is known about the exact mechanisms underlying this phenomenon. A better understanding of these mechanisms of neuroprotection can offer interesting targets for the development of new therapeutic approaches.

Currently, various animal models are being used to study $\mathrm{HI}$ preconditioning in the brain. Most studies have used adult stroke models, combining different types of artery occlusion (i.e. common carotid artery and middle cerebral artery) and exposure to low oxygen concentrations [1 18, 285-287]. There are, however, fewer models to study preconditioning in the immature brain. Gidday et al. reported that exposure of 7-day-old pups to $8 \%$ oxygen induced a robust neuroprotection against a stroke /combination of $\mathrm{FiO} 2$ of $8 \%$ and carotid occlusion) one day later [124]. Another model used is based on clamping of the uterine circulation at embryonic day 17 (E 17) followed stroke at postnatal day 7 (P7) [130]. Therefore, almost all recent preconditioning models in the immature brain aim to influence or modulate the effects of a neonatal stroke (i.e. focal insult). Focal insults, however, differ from the more relevant clinical situation at birth because they lack multi-organ injury, which is present in most infants with moderate or severe post-asphyctic encephalopathy and which may also influence the neurological outcome.

In the present study our aim was to develop a new model of fetal HI preconditioning by combining two global asphyctic insults which will reflect better the pathological conditions of asphyxia induced in a very early stage of brain development. For this purpose, the uterine vasculature of pregnant rats was clamped for 30 minutes on E17. On P0, the uterine horns still containing the fetuses were placed in a water bath to induce a second perinatal asphyctic insult. On P4 the reactivity of isolated carotid arteries was studied in a wire myo- 
graph. On P8, brains were analyzed for apoptotic cell death in the prefrontal cortex and striatum, using a terminal deoxynucleotidyl transferase-mediated dUTP-biotin nick end-labeling (TUNEL) staining. We hypothesize that fetal asphyxia (FA) may alter carotid artery responses and/or the brain damage induced by severe perinatal asphyxia group (SPA) in the rat.

\section{Materials and methods}

\section{Animals}

All experiments were approved by Animal Ethics Board of Maastricht University on animal welfare according to Dutch governmental regulations. Wistar rats, obtained form Charles River (Maastricht, The Netherlands), were kept under standard laboratory conditions with food and water given ad libitum, $21 \pm 2^{\circ} \mathrm{C}$ environment temperature, a $12 \mathrm{~h}$ light/dark schedule /lights on at 07:00 h) and background noise provided by radio. Exclusively male offspring were used within this study, because both morphological and behavioral evidence show a differential vulnerability to a birth insult in males versus females. A greater impact is seen in the male gender, probably due to the protecting role of the circulating estrogens in females [191, 192].

\section{Animal model}

The new preconditioning model is a combination of two previously well described models. The first one is a model for FA, used as a preconditioning stimulus, on E17, by completely clamping the uterine and ovarian arteries with removable clamps for 30 minutes. A second hit, SPA, was induced on E21/22 by submersing the uterine horns still containing the fetuses in a water bath, precisely calibrated at $37^{\circ} \mathrm{C}$, for exactly 19 minutes /starting from cutting off the blood circulation of the uterus to the moment the pups were taken from the water bath) $[57,130]$. The uterine horns were dissected quickly and the pups were removed, cleaned with medical swipes and stimulated manually to breathe to aid recovery within a pediatric incubator $\left(37^{\circ} \mathrm{C}, 60-80 \%\right.$ humidity, normal gas values). Pups of all conditions were left to recover for approximately 
60 minutes after birth in the same controlled environment. No more than 2 pups per litter were used to prevent litter effects. Afterwards, the pups were randomly cross-fostered to surrogate dams, each surrogate mother receiving 10 pups. Four groups of animals were used: FA, SPA, fetal asphyxia combined with severe perinatal asphyxia (FA+SPA) and control. Body weight of the pups was measured on P0 and P8 or P15, respectively.

\section{Vascular reactivity studies}

Common carotid arteries from P4 pups were isolated and kept in Krebs-Ringer bicarbonate (KRB) solution (in mmol/L: $\mathrm{NaCl} 118.5, \mathrm{MgSO}^{*}$ * $7 \mathrm{H} 2 \mathrm{O}$ 1.2, KH2PO4 1.2, NaHCO3 25.0, $\mathrm{KCl} 4.7, \mathrm{CaCl} 2.5$ and glucose monohydrate 5.5). Rings of $\sim 2 \mathrm{~mm}$ in length were mounted between an isometric force transducer (Kistler Morce DSC 6, Seattle, U.S.A.) and a displacement device in a myograph (model 610M, Danish Myotechnology, Aarhus, Denmark) using two stainless steel wires (diameter $40 \mu \mathrm{m}$ ). During mounting and experimentation, the KRB buffer was maintained at $37^{\circ} \mathrm{C}$ and aerated with $21 \%$ O2-74\% N2-5\% $\mathrm{CO} 2$. Artery rings were normalized to a resting pretension corresponding to an intraluminal pressure of $55 \mathrm{mmHg}$. Pilot experiments showed that at this initial passive tension the vessels developed maximal contraction in response to 62.5 $\mathrm{mM} \mathrm{K}+$. Vascular responses to $\mathrm{K}+(62,5 \mathrm{mM})$, the thromboxane $\mathrm{A} 2$ mimetic U46619 $(0.1 \mu \mathrm{M})$, the adrenergic receptor agonist norepinephrine (NE, 0.1$100 \mu \mathrm{M}$ ), the endothelium-dependent vasodilator acetylcholine (ACh 0.1-100 $\mu \mathrm{M})$, the NO-donor sodium nitroprusside (SNP, 0.1-100 $\mu \mathrm{M}$ ), the adenylate cyclase stimulator forskolin (0.1-10 $\mathrm{MM}$ ), and acute hypoxia were studied. For the study of the responsiveness to ACh, SNP, and hypoxia vessels were pre-contracted with U46619 (0.1 $\mu \mathrm{M})$. Hypoxia was induced by switching the bubbling gas mixture from $21 \%$ O2-74\% N2-5\% CO2 (pO2 $17 \pm 1 \mathrm{kPa}$ ) to 95\% N2-5\% CO2 (pO2 $2.3 \pm 0.4 \mathrm{kPa}$ ), as previously described [288].

\section{Evaluation of apoptotic cell death}

On P8, pups were anesthetized and perfused intracardially by fixative containing 4\% paraformaldehyde. Right hemispheres were entirely cut to serial, $30 \mu \mathrm{m}$-thick coronal cryostate sections. Sections were dried, defatted with a 
Triton-X100 solution and stained with cresyl violet. Volumes estimates of the striatum and prefrontal cortex were calculated according to the Cavalieri's principle [193]. This principle gives an unbiased estimate of the volume of the region of interest by multiplying the sum of the profile areas on all sections with the distance between the sections. The prefrontal cortex and the striatum were delineated as previously described by Kantor et al. [289] by their anatomical landmarks according to Paxinos and Watson [290]. Tracing was carried out with a stereology workstation and Stereolnvestigator software (MicroBrightField, Williston, VT).

Left hemispheres were cut into serial, $16 \mu$ m-thick coronal cryostate sections and cell death was identified by a terminal deoxynucleotidyl transferase-mediated dUTP-biotin nick end-labeling (TUNEL) staining, as described before by Van de Berg et al [58]. Briefly, sections were permeabilized by methacarn and then labeled with the reaction mixture in a humidified box at $37^{\circ} \mathrm{C}$ for $1.5 \mathrm{~h}$. This reaction mixture consisted of $0.1 \mu \mathrm{l} \mathrm{TdT}(400 \mathrm{U} / \mu \mathrm{l}) ; 1 \mu \mathrm{l}$ cobalt chloride $(2.5$ $\mathrm{mM}$ ); $2 \mu \mathrm{ml}$ TdT reaction buffer; $0.4 \mu$ l biotin-dUTP (2 nM); $6.5 \mu$ l MilliQ (Roche Diagnostics Nederland B.V., Almere, Netherlands). The reaction was stopped by placing the slides in $4 x$ standard saline citrate buffer. Next, streptavidin-Alexa 594 ( 1:2000 dilution; for 60 min at RT, Sigma, The Netherlands) was used to visualize nuclei labeled with biotinylated dUTP. Sections were counterstained with Hoechst 33342 (1:500 dilution; 30 min at RT; Sigma, The Netherlands). The procedure was finished by washing and enclosure with TBS:glycerol (1:3). TUNEL-positive cells were counted in the two regions of interest /striatum and prefrontal cortex), using the counting method described by Van de Berg et al. [58]. To detect the presence of positive cells, all slices were examined at a magnification 40x with an Olympus AX-70 microscope. Every TUNEL-positive cell, characterized by its intense red staining, was double-checked with the Hoechst 33342 staining to seen whether that particular cell was highly pyknotic and whether the nucleus was fragmented.

\section{Statistical analysis}

Results are shown as means (SD) of measurements in $n$ animals. For clarity, results in the figures are shown as means +/- SEM. Vascular contractions are ex- 
pressed in terms of active wall tension $(\mathrm{N} / \mathrm{m})$, calculated as the force divided by twice the length of the arterial segment, while the relaxant responses are expressed as the percentage of reduction of the contraction induced by U46619. Sensitivity (expressed as pD2 $=\log$ EC50) and maximal response (Emax) to agonists was determined for each artery by fitting individual concentration-response data to a non-linear sigmoidal regression curve (Graphpad Prism version 5.01; GraphPad Software Inc, San Diego, U.S.A.). Apoptotic cell death results are expressed in terms of mean total number of TUNEL-positive cells per area as well as density of TUNEL-positive cells per area. Qualitative variables were compared using the Fisher exact test and continuous variables through analysis of variance (ANOVA) followed by a Bonferroni post hoc test. Differences were considered to be significant if $P<0.05$. All calculations were done using the Statistical Package for the Social Sciences (SPSS 15.0 software).

\section{Results}

\section{Survival}

$55 \%$ of the SPA animals died at birth, while none of the FA or control animals died. When the pups had a prenatal $\mathrm{HI}$ exposure before being exposed to a perinatal insult, fewer animals died (FA+SPA $=36 \%)$. This difference in mortality rate between the SPA and the FA+SPA did not reach statistical significance $(P=0.107 ; N=243$ pups and 25 dams). Moreover, a few pups died in the period between P0 and P15. These differences were, however, not significant. It is also important to note that none of the fetuses died intrauterinely in the period between the FA insult at E17 and birth.

\section{Somatic growth}

Birth and body weights are presented in figure 2. One-way ANOVA analysis showed an overall effect on birth weight (Figure 2A). Post-hoc Bonferroni demonstrated that both SHAM (-9\%, $p<0.01)$ and FA (-11\%; $p<0.01)$ pups had significantly lower birth weights than control animals that did not undergo a procedure before birth (CVD, CCD and SPA). Mean body weights were meas- 
ured at $\mathrm{P} 7$ and $\mathrm{P} 14$ (Figure 2B). A repeated measured ANOVA showed a significant overall effect of both time $(p<0.001)$ and group $(p<0.001)$. At $P 7$, SHAM/SPA $(p<0.05)$ and FA/SPA $(p<0.05)$ animals were significantly lighter than CVD and CCD animals. At P14, SHAM ( $p<0.05)$, FA ( $p<0.05)$, SHAM/SPA $(p<0.05)$ and FA/SPA $(p<0.05)$ animals were significantly smaller than the CVD, CCD and SPA animals.
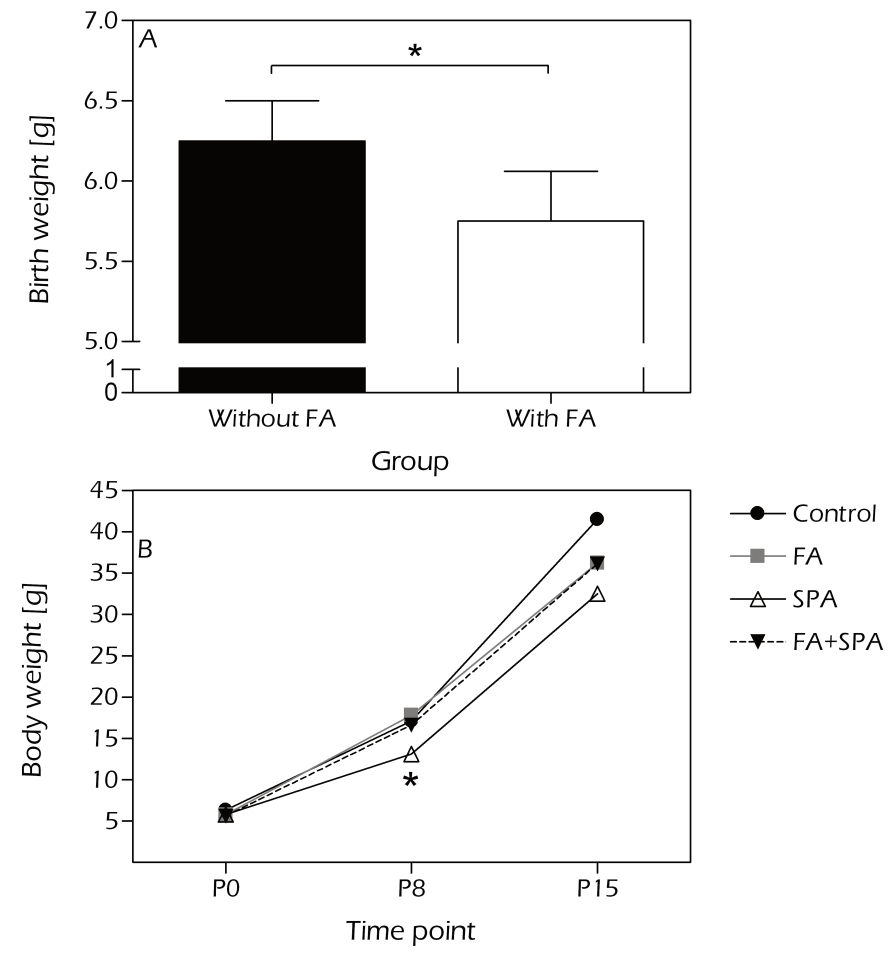

Figure 5: The birth and body weights of the different groups on the different time points.

(A) Birth weight of the pups. The pups that underwent a FA insult, meaning both the FA and the FA+SPA group, have a smaller birth weight than the pups that did not undergo a FA insult (control and SPA group). (B) Body weights of the pups at the different time points during the experiment. On $P 8$, the mean body weight of the SPA group was lower compared to the other groups. $N=4$ animals per group. Data are expressed as mean + SEM. (ANOVA + Bonferroni; * $p<0.05)$. 


\section{Reactivity of carotid arteries}

Potassium (62,5 mM) and U46619 (0.1 $\mu \mathrm{M})$ induced tonic contractions in neonatal carotid arteries (Figure 2A). In contrast, NE induced very weak contractions (Control Emax 0.025N/m, SD 0.030, n=16). To asses whether this hyporesponsiveness to NE was an age-related feature, we performed concentration-response curves to NE in carotid arteries from adult rats (18-wkold) and observed a high contractile potency and efficacy of the adrenergic agonist (Figure $2 \mathrm{~B}$ and $2 \mathrm{C}$ ). The contraction induced by $\mathrm{K}+$ was significantly reduced in the SPA group (0.067 N/m, SD 0.072, n=14) when compared with the control $(0.182 \mathrm{~N} / \mathrm{m}, \mathrm{SD} 0.147, \mathrm{n}=37)$ and the FA+SPA $(0.177 \mathrm{~N} / \mathrm{m}, \mathrm{SD}$ $0.117, n=18$ ) groups (Figure 2A). The contractions induced by U46619 were not significantly different in the four groups (Figure 2A).

ACh (Figure 3A), SNP (Figure 3B) and forskolin (Figure 3C) relaxed U46619contracted neonatal carotid arteries in a concentration-dependent manner. The maximal relaxation induced by ACh was significantly higher in the FA group (Emax 96.79\%,SD 70.08, $n=5$ ) than in the control (Emax 41.65\%, SD $18.99, \mathrm{n}=18$ ) and the FA+SPA (Emax 38.53\%, SD 24.60, $\mathrm{n}=10$ ) groups (Figure $3 A$ ). Sensitivities to $A C h$ were comparable in the four groups ( $P D 2$ control: 6.62, SD 0.46; FA: 6.48, SD 0.15; SPA: 6.49, SD 0.72; FA+SPA: 6.45, SD 0.72). The relaxations induced by SNP and forskolin were not significantly different in the four groups.

Acute hypoxia relaxed U46619-induced contraction (Figure 3D). The maximal relaxations observed after 10 minutes of hypoxia were not significantly different in the four groups (control: 28.99\%, SD 22.75; FA: 65.28, SD 79.68; SPA: 16.13, SD 57.31, FA + SPA: 32.30, SD 39.73). Also when the relaxation induced by 10 min of hypoxia was expressed as area above the curve /corrected for the U46619-induced contraction) no significant differences among the four groups were observed (Figure 3E). 

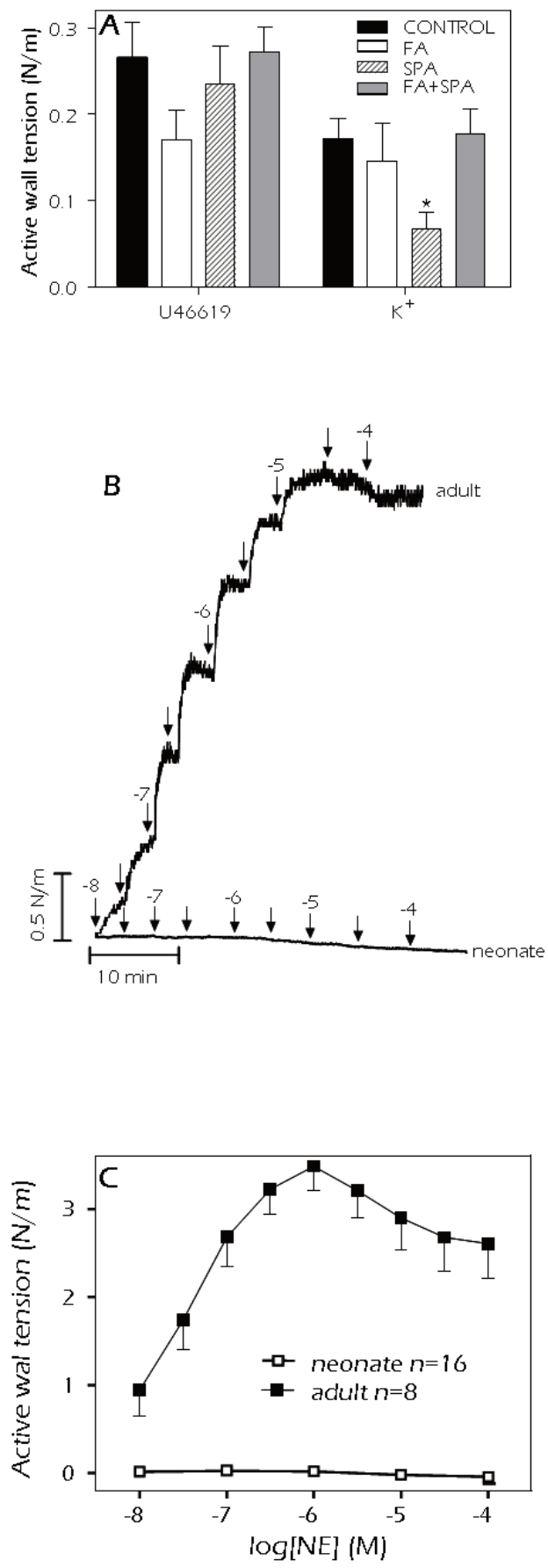

Figure 2: Contractions induced by K+, U46619 and norepinephrine in neonatal (P4) carotid arteries.

(A) The contraction induced by $\mathrm{K}+(62,5 \mathrm{mM})$ was significantly reduced in the SPA group $\left({ }^{*} \mathrm{P}<0.05\right)$ when compared with the control (CON) and the FA+SPA groups. The contractions induced by U46619, a thromboxane A2 mimetic $(0.1 \mu \mathrm{M})$, were not significantly different in the four groups. (B) Representative tracing showing the contractile effects of NE in neonatal and adult (18-wk-old) carotid arteries. (C) Summary of the contractile effects of the adrenergic receptor agonist, norepinephrine (NE, 0.1-100 $\mu \mathrm{M}$ ), in neonatal and adult carotid arteries. 

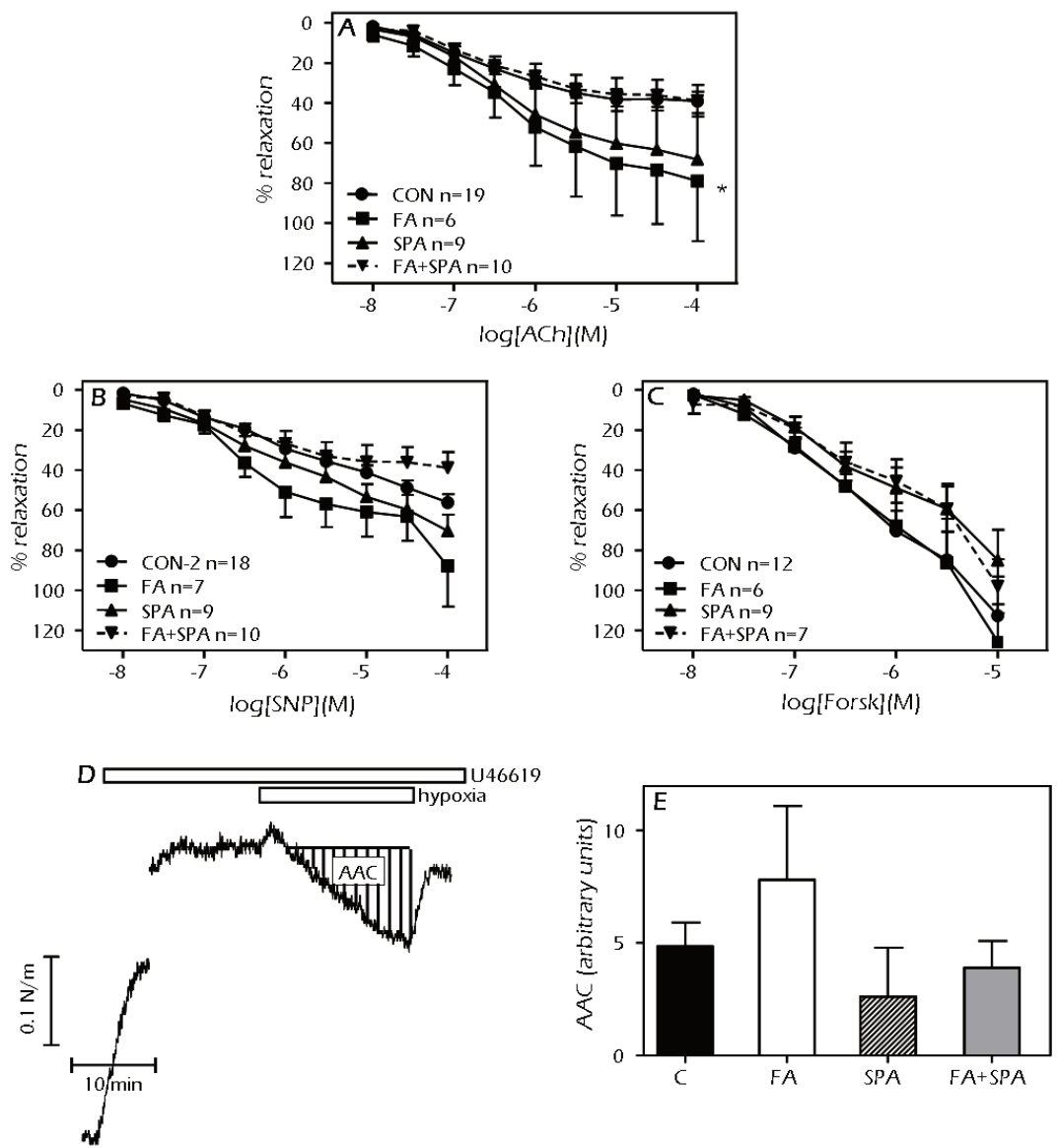

Figure 3: The contraction-dependent relaxant effects

(A-C) Concentration-dependent relaxant effects of the endothelium-dependent vasodilator acetylcholine (ACh, A), the NO-donor sodium nitroprusside (SNP, B), and the adenylate cyclase stimulator forskolin (C) in neonatal (P4) carotid arteries pre-contracted with U46619 (0.1 $\mu \mathrm{M})$. The maximal relaxation induced by ACh was significantly higher ( $\left.{ }^{*} \mathrm{P}<0.05\right)$ in the FA group than in the control (CON) and the FA+SPA groups. (D) Representative tracing showing the effects of a 10 min hypoxic challenge (switching the gas mixture in the organ chamber from 21\% O2-74\% N2-5\% CO2 to 95\% $\mathrm{N} 2-5 \% \mathrm{CO} 2)$ in a neonatal carotid artery pre-contracted with U46619 (0.1 $\mu \mathrm{M})$. (E) Summary of the relaxant responses to acute hypoxia (expressed as area above the curve, $A A C$ ) in the four experimental groups. 


\section{Apoptotic cell death (TUNEL)}

No gross morphological changes were seen in the brain of asphyctic pups compared with control pups 8 days after delivery. Typical apoptotic morphological changes like condensation, shrinkage and fragmentation were found in all four groups and were visible with both the TUNEL and the Hoechst staining (Figure 4). TUNEL stained cells were mainly found in the area surrounding the lateral ventricle and the corpus callosum and in the dorsal part of the striatum. They mostly lay together in clusters, showing a patchy pattern.

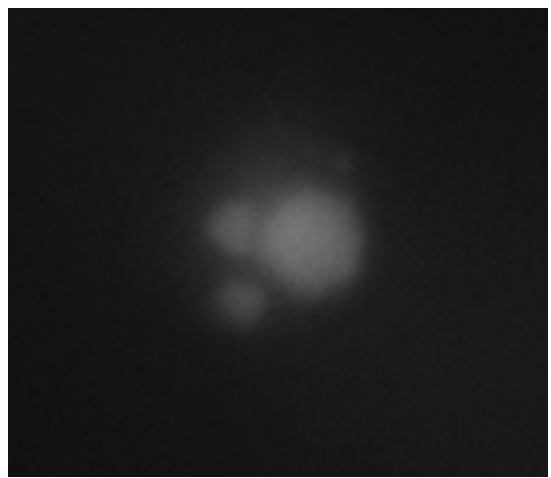

Figure 4: A representing photograph of a TUNELstained cell. This picture was taken at a high magnification (100x) and shows the defragmentation of the nucelus.

Figure 5 summarizes the results of the quantitative analysis of the TUNEL stained cells and the volume estimates in the striatum and prefrontal cortex. Two-way ANOVA analysis revealed no significant changes in either striatal or prefrontal cortex volume between groups (Figures 5B and 5E). Two-way ANOVA analysis also revealed a significant increase in the total number of apoptotic cells due to a FA insult (FA and FA+SPA group) in both areas investigated ( $p=0.03$ and $p=0.04$, respectively). However, post-hoc Bonferroni testing did not detect any significant differences between the FA and FA+SPA groups and the control group (Figures 5A and 5D). In both striatum and prefrontal cortex, SPA also significantly increased the total number of apoptotic cells (two-way ANOVA: $p=0.01$ and $p=0.02$, respectively). This difference could 
be almost completely attributed to the SPA (Post-hoc Bonferroni; control vs SPA: $p=0.01$ and $p=0.01$, resp.; $+127 \%$ and $+133 \%$, resp.), and not to FA+SPA group. Brains subjected to FA before undergoing SPA demonstrated a significant reduction in apoptotic cells in the striatum (Post-hoc Bonferroni; $p<0.001$; $-46.4 \%$ ) and prefrontal cortex (Post-hoc Bonferroni; $p=0.003 ;-44 \%$ ) compared to brains only subjected to SPA.

Taking into account both the data of the TUNEL-positive cell counting and the volume measurements allowed us to estimate the density of apoptotic cells (Figures 5C and 5F). FA caused a small increase in the mean density of apoptotic cells in the striatum and the prefrontal cortex (Two-way ANOVA: $p=0.02$ and $p=0.04$, resp.). However, post-hoc Bonferroni analysis did not find any significant differences between the FA group and the control group. Two-way ANOVA also revealed a significant increase in the mean density of apoptotic cells due to SPA in both areas ( $p<0.001$ and $p=0.003$, resp.), which could be almost entirely ascribed to the SPA group (Post-hoc Bonferroni; control vs SPA: $p<0.001$ and $p<0.001$, resp.; $+138 \%$ and $+166 \%$ ). Moreover, post-hoc testing demonstrated that the mean density of apoptotic cells in the FA+SPA group was significantly lower than in the SPA group in the striatum $(p<0.001 ;-33 \%)$ as well as the prefrontal cortex $(p=0.007 ;-47 \%)$.

\section{Discussion}

Asphyxia during development is a major cause of neonatal mortality and of lifelong neurological disabilities among survivors. Although recent evidence suggests that moderate hypothermia (core temperature $33-34^{\circ} \mathrm{C}$ for 72 hours) im $\mathrm{mprov}_{\neg}$ es the outcome of term newborns that suffered from moderate postasphyctic encephalopathy [291], there are still no other clinically proven neuroprotective agents available for these newborns. Revealing the mechanisms behind $\mathrm{HI}$ preconditioning and tolerance could therefore be an important step towards an effective therapy. We aimed to determine whether a fetal asphyctic insult protects against a subsequent more severe perinatal asphyctic insult. Both insults used are global events that are associated with both systemic metabolic and hemodynamic changes in the fetus that may influence the outcome 

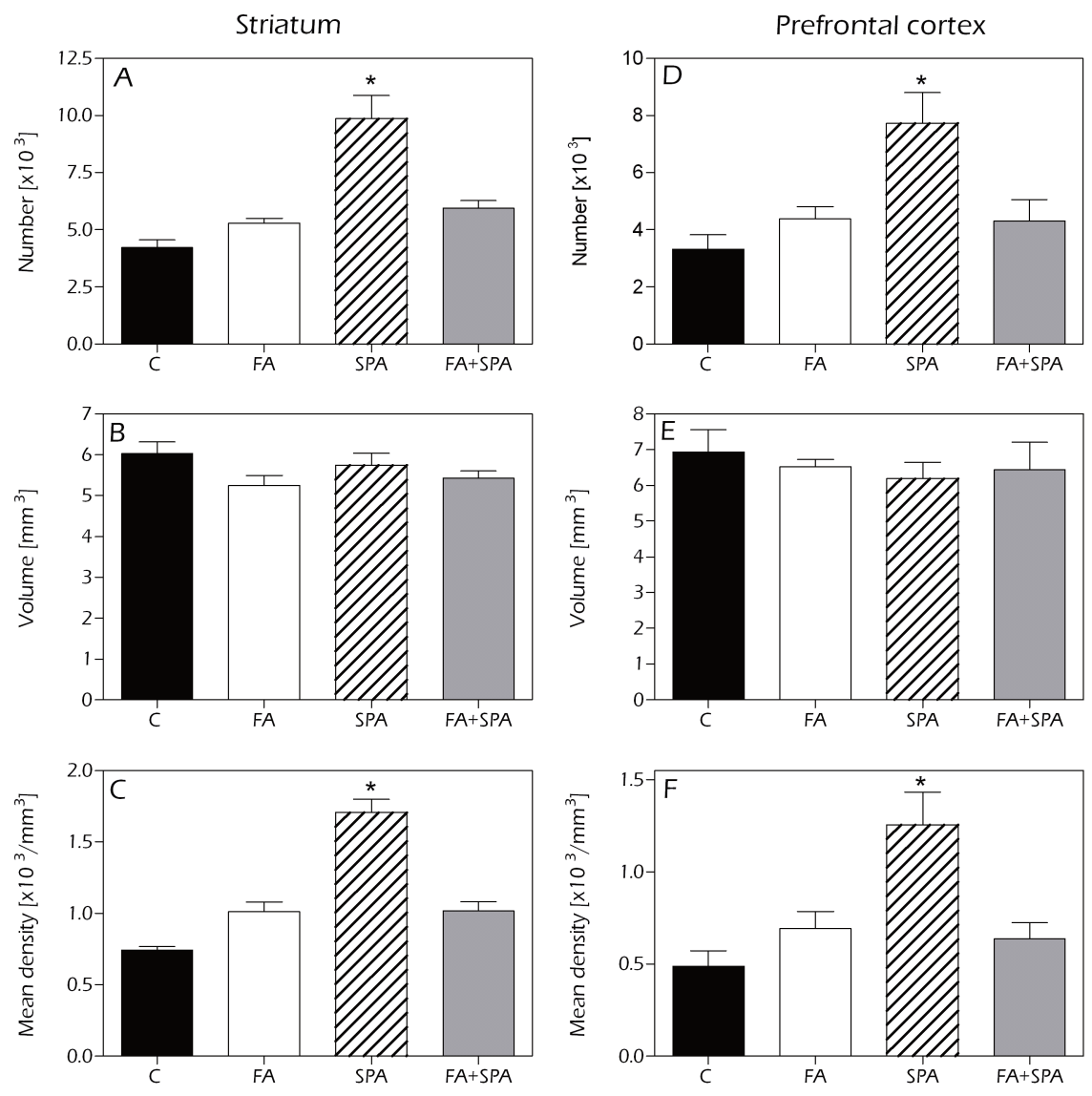

Figure 5: Quantitative analysis of TUNEL-positive cells and volume measurements of the striatum and the prefrontal cortex 8 days after the severe perinatal insult.

(A) The total number of apoptotic cells in the striatum $\left(\times 10^{3}\right)$. There is a significant increase in the number of TUNEL-positive cells in the striatum of SPA animals compared to the other groups. In addition, FA significantly decreased the number of apoptotic cells after SPA. (B) Striatal volume estimates $\left(\mathrm{mm}^{3}\right)$. There was no significant difference in striatal volume between groups. (C) The mean density of apoptotic cells in the striatum $\left(\times 10^{3} / \mathrm{mm}^{3}\right)$. The mean density of TUNEL-positive cells was higher in the SPA group than in the other groups. Furthermore, this density was significantly lower in the FA+SPA group compared to SPA group. (D) The total number of apoptotic cells in the prefrontal cortex $\left(\times 10^{3}\right)$. SPA group had a significantly more TUNEL-positive cells than the other groups. FA+SPA pups had less TUNEL-positive cells than pups only subjected to SPA. (E) Volume estimates of the prefrontal cortex $\left(\mathrm{mm}^{3}\right)$. The prefrontal cortex volume estimates did not differ between groups. $(\mathrm{F})$ The mean density of apoptotic cells in the prefrontal cortex $\left(\times 10^{3} / \mathrm{mm}^{3}\right)$. The SPA group significantly higher the mean density of TUNEL-positive cells compared to the other three groups. Additionally, FA lowered the mean density of TUNEL-positive cells after SPA. N = 4 animals per group. Data are expressed as mean + SEM. (ANOVA + Bonferroni; * $p<0.05)$. 
of the animal. These changes mimic the global and pathophysiological conditions of asphyxia induced before and during birth. Therefore, they reflect the clinical situation more closely than stroke insults. In the present study, we evaluated the effects of this novel model of preconditioning on carotid vascular reactivity and cerebral apoptotic cell death.

\section{Vascular reactivity studies}

Following severe perinatal asphyxia the autoregulatory ability of the neonatal cerebral vascular bed is impaired $[292,293]$. We aimed to analyze whether this impairment was reflected in alterations of neonatal carotid arteries reactivity. The carotid arteries play an important role in responses to hypoxia, particularly in the fetal and neonatal period, where the regulatory capacity of the small cerebral arteries is functionally immature [294]. We have observed that neonatal (P4) rat carotid arteries are sensitive to receptor-independent (high $\mathrm{K}+$ ) and -dependent (U46619) contractile agonists as well as to endotheliumdependent (ACh) and -independent (SNP and forskolin) relaxant agonists. Moreover, carotid responsiveness to acute hypoxia was also present at this age. Interestingly, the P4 carotid arteries did not respond to the adrenergic agonist $\mathrm{NE}$, which was a potent contractile agent in the adult carotid arteries.

The switch from the proliferative to the mature contractile vascular phenotype is an important developmental event whose timing appears to be vessel and species specific [295-297] . This process include maturational changes in contractile proteins, ion channels, endothelial cell function, vessel wall growth and receptor expression. In rodents an important part of these maturational changes appears to occur postnatally [295], whereas it begins before birth in sheep [296]. Accordingly, several studies demonstrated adrenergic-mediated contractions in isolated carotid arteries from fetal and neonatal lambs [296, 298] [299]. In fetal and neonatal rat carotid arteries, mounted in a pressurized myograph system, adrenergic agonists induced some reduction in lumen diameter [300, 301]. However our present data indicate that these diameter changes are probably not accompanied by a significant increase in carotid wall tension. In contrast, the vascular contractile machinery was mature to respond to non-adrenergic agonists. Fetal and perinatal asphyxia are stressors that elicit integrated cardiovascular and endocrine responses producing a redistribution 
of the combined ventricular output in favor of the adrenal, myocardial, and cerebral circulations [302]. Rat and lamb models are the most frequently used in the study of the pathophysiology of fetal and perinatal asphyxia. The differential maturation in vascular responsiveness between the two species, particularly in adrenergic responsiveness, should be taken into account when interpreting the flow redistribution response induced by asphyxia.

When we analyzed the effects of fetal and perinatal asphyxia on carotid reactivity, we observed a reduced receptor-independent contraction in the SPA group and an increased endothelium-dependent relaxation in the FA group. A similar trend, although not significant, toward increased endothelium-dependent relaxation was observed in the SPA group. Interestingly, when the animals were exposed to fetal and perinatal asphyxia, carotid artery responsiveness was similar to the observed in the control group. These findings indicate that functional changes occur in neonatal vascular reactivity following asphyxia, a result that may significantly influence asphyctic damage by altering blood flow to the brain. Endothelium-derived nitric oxide (NO) plays an important role in the autoregulation of cerebral circulation and numerous experimental evidences indicate its involvement in post HI brain injury [292, 303-308]. Excessive NO production leads to damage to the cerebral microcirculation and directly to brain tissue [303, 308, 309]. In addition, immediate postischemic cerebral hyperperfusion appears to be mediated, at least partially, by increased NO production and postischemic reperfusion damage to the brain can be reduced by NO synthase blockade [292, 304-307]. Our present data suggest that exposure to fetal asphyxia-induced preconditioning might modulate the NO regulatory ability on post $\mathrm{HI}$ cerebral blood flow.

\section{Apoptotic cell death}

To evaluate the putative brain protective effect of fetal asphyctic preconditioning, the number of apoptotic cells was analyzed in the striatum and prefrontal cortex of 8-day-old rat pups, using a TUNEL staining. The TUNEL method detects nuclear DNA fragmentation in cells. Although fragmentation is a pattern typical of apoptosis, it can also occur, to a lesser extent, in necrotic cells. As a consequence, TUNEL can sometime also stain necrotic cells. Because 
each stained cell was analyzed for apoptotic traits like nuclear fragmentation and a pyknotic nucleus with a Hoechst staining, we assume that the majority of counted cells were apoptotic.

Only a few apoptotic cells were found in the control and FA group. This kind of apoptosis probably represents naturally occurring developmental cell death. The SPA group showed a substantial increase in TUNEL-positive cells in both the striatum and prefrontal cortex. Pups that underwent FA before being subjected to SPA had significantly less apoptotic cells than pups that did not undergo FA, suggesting a protective effect. Their number of apoptotic cells was similar to that of the control and FA groups. Since the results were similar for both areas investigated (i.e. prefrontal cortex and striatum), it seems that both the damage and the protection represent a global effect. Another important finding of this study is that the SPA group showed a growth retardation which was absent in the FA+SPA group. Since the FA insult was global, this protection may include protective effects of many organs and systems, such as the heart and the arteriovenous system. This awaits further research though.

Although this study is the first to look at HI preconditioning in a global asphyxia model, our current findings are consistent with cell protection by HI preconditioning against focal $\mathrm{HI}$ injury (i.e. stroke models). Cantagrel et al. found that exposure of the pregnant dam to a hypoxic gas mixture $(8 \% 02 / 92 \% N 2)$ causes a reduction in the number of apoptotic cells in the striatum, hippocampus and cortex $24 \mathrm{~h}$ and $48 \mathrm{~h}$ after right carotid ligation on postnatal day 7 (Rice-Vannucci model) [126]. In the same rat model, Zhao et al. demonstrated that newborns subjected to a sublethal prenatal asphyxia show lower mortality rates, less brain tissue damage, less neuronal loss and fewer caspase-3 positive cells after stroke [140]. Similarly, Cai et al. demonstrated that a prenatal HI insult, achieved by clamping the ovarian and uterine arteries for $30 \mathrm{~min}$, also reduces the brain infarct size and neuronal injury induced by the Rice-Vannucci model [130].

An important remark to be made is that the neuroprotective effects induced by fetal asphyxia in this study may be partly due to the anesthetics (i.e. isoflurane). Kersten et al. reported that isoflurane can mimic the protecting effects of preconditioning in the rat myocardium, while Kapinya et al showed that isoflurane induces prolonged protection against cerebral ischemia [310, 311]. 
Moreover, Caesarean section per se induces specific neurobehavioral alterations with respect to, for example, response to stress. Furthermore, the addition of a period of asphyxia worsens some of the behavioral changes produced by C-sections alone. Consequently, isoflurane and C-section can possibly influence the results of apoptotic cell counts. For that reason, it is important to include vaginally-delivered as well as SHAM-operated control animals in further studies.

\section{Final remarks}

Animals exposed to a brief period of global FA seem to be protected against the vascular alterations and the increased apoptotic cell death in striatum and prefrontal cortex, following SPA 4 days later. This FA preconditioning is most likely the result of the delayed phase of neuroprotection, which requires new protein synthesis and gene expression. This anti-apoptotic effect probably involves several molecules, as described above, that are induced and regulated as part of a complex. To imply the role of these molecules in FA-induced tolerance, further experiments are required. Identifying these mechanisms could provide rational targets for future pharmacological intervention. 

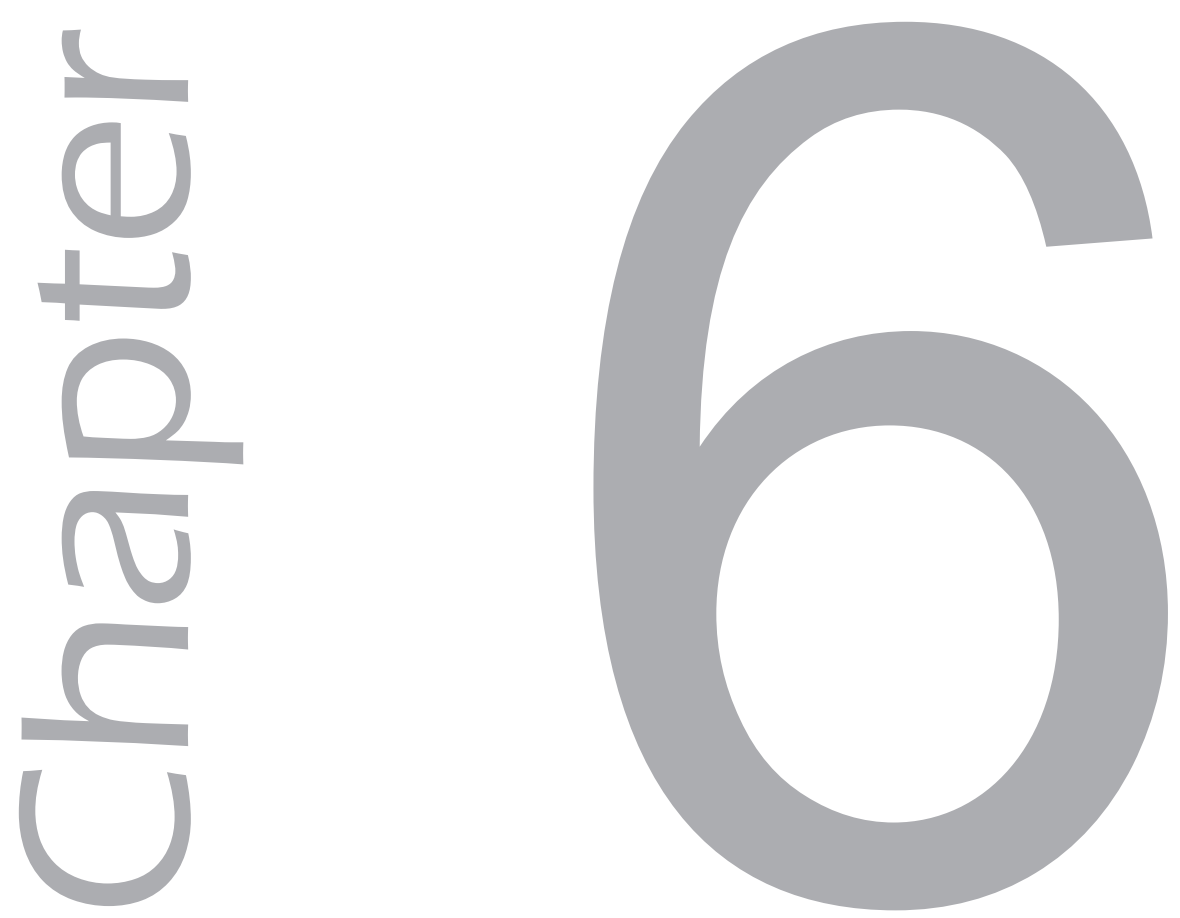

\section{Fetal asphyctic preconditioning protects against perinatal asphyxia-induced apoptosis and astrogliosis in the neonatal brain}

E.Strackx, K.E.M. Cox, D.L.A. Van den Hove, J.R.A. Van Ekkendonk, L.J.I. Zimmermann, H.W.M. Steinbusch, C.E. Blanco, J.S.H. Vles and A.W.D. Gavilanes 


\begin{abstract}
Hypoxic-ischemic preconditioning is a protection mechanism in which exposure to a sublethal episode of hypoxia-ischemia protects the brain against a subsequent more severe episode. Although a lot of postnatal and adult preconditioning models exist, very few pre- and perinatal models have been established. Therefore, the objective of this study was to develop and validate a new model to investigate fetal asphyctic preconditioning in the neonatal brain. We examined whether mild fetal asphyxia (FA), as a preconditioning stimulus, can protect against severe perinatal asphyxia (SPA). FA was induced at embryonic day 17 by completely clamping the uterine and the ovarian arteries for 30 minutes. Four days later, at postnatal day 0, SPA is provoked by submersing the uterine horns still containing the fetuses in a water bath for 19 minutes. At postnatal day 7, rat brains were examined for SPA-induced morphological changes. For that reason, sections were stained with a terminal deoxynucleotidyl transferase-mediated dUTP-biotin nick end-labeling (TUNEL) staining to detect apoptotic cell death and with a glial fibrillary acidic protein (GFAP) staining to detect reactive astrocytes. Design-based stereology was used to count TUNEL- and GFAP-positive cell numbers. Results showed that the preconditioned group showed significantly less perinatal mortality than the non-preconditioned group. Furthermore, the preconditioned animals weighed less at birth in comparison to control animals. The most important finding of this study is, however, that the preconditioned animals had a significantly lower number and density of TUNEL-positive cells in the striatum, frontoparietal cortex and hippocampus compared to the non-preconditioned animals. In addition, the preconditioned animals also had a decreased number and density of GFAP-positive astrocytes in those areas. Taken together, this study demonstrates FA preconditioning decreases the number of apoptotic cells and the number of astrocytes compared to non-preconditioned animals. Consequently, mild FA might cause neuroprotection by inducing anti-apoptotic mechanisms and by attenuating astrogliosis.
\end{abstract}



postnatally, is a major cause of neonatal mortality and morbidity $[6,161,284]$. It is frequently associated with short and long-term neurological dysfunction, like motor disabilities and learning and cognitive problems [259, 260]. The first hypothermia clinical trials seem promising. Nevertheless, meta-analysis of those trials using post-asphyctic mild-moderate hypothermia for full-term infants show a mean number needed to treat of 6 . Therefore, there is an urgent need to develop additional neuroprotective strategies that may, whether or not combined with hypothermia, provide an even better tolerance $[2,107,109,113,114,312]$.

An interesting approach to further study neuroprotection is investigating the physiological phenomenon of preconditioning. Virtually any stimulus capable of causing injury to a tissue or organ can, when applied sublethally, activate endogenous defensive mechanisms that protect against subsequent more severe injury. This phenomenon was first described in the heart by Murry and colleagues in 1986 [115]. They showed that a series of short coronary artery occlusions followed by reperfusion can protect the heart against a subsequent lethal ischemia. Since then, different models have been established to investigate this type of tolerance in various animals and organs. In the immature brain, Gidday and colleagues were the first to report neonatal preconditioning in 1994 [124]. Exposure of a 6-day-old rat pup to hypoxia (8\% FiO2 for 3h) was used as a preconditioning stimulus, followed by carotid artery occlusion one day later. They have shown that this type of preconditioning can reduce the infarct volume in the immature brain. Other studies using similar animal models confirmed these results [55]. A lower incidence in brain damage, a reduction in lesion volume and a decrease in the number of apoptotic cells in areas like the cortex, hippocampus and striatum were the main findings $[119,125,126$, $128,129]$.

Although a lot of postnatal and adult preconditioning models exist nowadays, no pre- and perinatal models have been established trying to provide protection against asphyxia during birth. Nevertheless, unique physiological mechanisms specific to the time of birth may play an important role in the development of post-asphyctic brain injury and/or neuroprotection. Additionally, the existing neonatal models all use the Rice-Vannucci model as the detri- 
mental insult. This stroke model only causes one-sided focal brain damage, which does not reproduce the most common pathological type of global asphyctic encephalopathy seen in the human neonate [168]. For those reasons, the objective of this study was to develop and validate a new model for preconditioning in the neonatal period, i.e. fetal asphyctic preconditioning. Mild fetal asphyxia was induced, as a preconditioning stimulus, by completely clamping the uterine and ovarian arteries for 30 minutes. Four days later, severe perinatal asphyxia was provoked by submersing the uterine horns, still containing the fetuses, in a water bath for 19 minutes. Both procedures better mimic the global, pathophysiological conditions of asphyxia before and during birth. Since the immature brain is known to be prone to especially apoptotic cell death, we chose to validate the model by investigating the number of apoptotic cells. Previous literature shows that in addition to developmental apoptosis, severe perinatal asphyxia-induced apoptosis peaks after 7 days [58, 59]. Therefore, this study investigated the number of TUNEL-positive cells in 7-dayold preconditioned and non-preconditioned rat pups. In addition, astrocyte numbers were assessed using a GFAP antibody to detect astrogliosis.

\section{Materials and methods}

\section{Animals}

All experiments were approved by Animal Ethics Board of Maastricht University on animal welfare according to Dutch governmental regulations. SpragueDawley rats, obtained form Charles River (Maastricht, The Netherlands), were kept under standard laboratory conditions with food and water given ad libitum, $21 \pm 2^{\circ} \mathrm{C}$ environment temperature, a $12 \mathrm{~h}$ light/dark schedule (lights on at 07:00 h) and background noise provided by radio. Exclusively male offspring were used within this study, because both morphological and behavioral evidence show a differential vulnerability to a birth insult in males versus females. A greater impact is seen in the male gender, probably due to the protecting role of the circulating estrogens in females [191, 192]. 


\section{Animal model}

Fetal asphyxia (FA) was used as a preconditioning stimulus on E17. FA was induced by completely clamping the uterine and ovarian arteries with removable clamps for 30 minutes. A second hit, severe perinatal asphyxia, was induced on E21/22 by submersing the uterine horns still containing the fetuses in a water bath, precisely calibrated at $37^{\circ} \mathrm{C}$, for exactly 19 minutes (starting from cutting off the blood circulation of the uterus to the moment the pups were taken from the water bath) $[57,130]$. The uterine horns were dissected quickly and the pups were removed, cleaned with medical swipes and stimulated manually to breathe to aid recovery within a pediatric incubator $137^{\circ} \mathrm{C}$, $60-80 \%$ humidity, normal gas values). Pups of all conditions were left to recover for approximately 60 minutes after birth in the same controlled environment. No more than 2 pups per litter were used to prevent litter effects. Afterwards, the pups were randomly cross-fostered to surrogate dams, each surrogate mother receiving 14 pups (2 pups of each condition). Seven groups of animals were used as depicted in table 1. Body weight of the pups was measured on $\mathrm{PO}$ and $\mathrm{P} 8$ or P15, respectively.

Table 1: Overview of the different experimental groups

\begin{tabular}{lccc}
\multicolumn{1}{c}{ Experimental group } & N & Intervention time & Abbreviation \\
\hline Control vaginal delivery & 8 & $/$ & CVD \\
\hline Control Caesarian delilvery & 7 & E21-22 & CCD \\
\hline SHAM-operated & 8 & E17 & SHAM \\
\hline Fetal asphyxia & 8 & E17 & FA \\
\hline Severe perinatal asphyxia & 8 & E21-22 & SPA \\
\hline SHAM + perinatal asphyxia & 7 & E17 + E21-22 & SHAM/SPA \\
\hline Fetal asphyxia + perinatal asphyxia & 8 & E17 + E21-22 & FA/SPA \\
\hline
\end{tabular}

Abbreviations: N: number; E: embryonic day

\section{Tissue preparation}

On P8, pups were anesthetized and perfused intracardially by fixative containing 4\% paraformaldehyde. Right hemispheres were entirely cut to serial, $30 \mu \mathrm{m}$-thick coronal cryostate sections. Sections were dried, defatted with a 
Triton-X100 solution and stained with cresyl violet. Left hemispheres were cut into serial, $16 \mu \mathrm{m}$-thick coronal cryostate sections to identify cell death.

\section{Immunohistochemistry}

Cell death was identified by a terminal deoxynucleotidyl transferase-mediated dUTP-biotin nick end-labeling (TUNEL) staining, as described before by Van de Berg et al [58]. Briefly, sections were permeabilized by methacarn and then labeled with the reaction mixture in a humidified box at $37^{\circ} \mathrm{C}$ for $1.5 \mathrm{~h}$. This reaction mixture consisted of $0.1 \mu \mathrm{l} \mathrm{TdT}(400 \mathrm{U} / \mu \mathrm{l}) ; 1 \mu \mathrm{l}$ cobalt chloride $(2.5 \mathrm{mM})$; $2 \mu \mathrm{l}$ TdT reaction buffer; $0.4 \mu \mathrm{l}$ biotin-dUTP $(2 \mathrm{nM}$ ); $6.5 \mu \mathrm{l}$ MilliO (Roche Diagnostics Nederland B.V., Almere, Netherlands). The reaction was stopped by placing the slides in $4 x$ standard saline citrate buffer. Next, streptavidin-Alexa 594 (1:2000 dilution; for 60 min at RT, Sigma, The Netherlands) was used to visualize nuclei labeled with biotinylated dUTP. Sections were counterstained with Hoechst 33342 (1:500 dilution; 30 min at RT; Sigma, The Netherlands). The procedure was finished by washing and enclosure with TBS: glycerol (1:3). Every TUNEL-positive cell, characterized by its intense red staining, was doublechecked with the Hoechst 33342 staining to seen whether that particular cell was highly pyknotic and whether the nucleus was fragmented.

Astrocytic changes were visualized using glial fibrillary acidic protein (GFAP) as a marker. Cryostat sections were labeled with polyclonal rabbit anti-GFAP (1:1600; Innogenetics), using donkey anti-rabbit biotinylated immunoglobulins as secondary antibodies (1:200; Jackson). Brain sections were further processed for immunohistochemistry using the avidin biotin complex technique with 3,3-diaminobenzidine (DAB) to obtain a color reaction (Vector laboratories, USA).

\section{Design-based stereology}

Design-based stereology was performed for volume measurements and cell counts, using a stereological computer microscopy system and Stereolnvestigator software (MBF Bioscience, Williston, VT, USA). The regions of interest (e.g. striatum, prefrontal cortex, parietal cortex and hippocampus) delineated 
according to Paxinos and Watson (1986) by anatomical landmarks with a $1.25 x$ magnification on live microscopic video images displayed on a monitor. Volumes were estimated by multiplying the average surface area with the section thickness, the number of sections investigated, and a factor of seven since every eighth section was used (section sampling fraction (ssf) = 1/8) [193].

The TUNEL and GFAP-positive cells were counted with the optical fractionator method [193]. Briefly, counting frames were created by the software and placed at the intersections of a grid that had been randomly placed over the section. The counting frames were replaced systematically by stepwise movements in $\mathrm{x}$ - and $\mathrm{y}$-directions. The area of the counting frame relative to the area associated with the $x$ and $y$ steps gives the area sampling fraction (asf). The optical disector height, along the z-axis, was determined by excluding the parts close to the slide and the coverslip. The height of the optical disector relative to the actual thickness of the section results in the height sampling fraction (hsf). Only counting frames for which at least a part of the frame fell within the delineated contour were used for counting. Cells were marked if they were positive and in focus within the counting area. Furthermore, the counting frames have forbidden lines to avoid edge effects. The estimated total number of positive cells ( $N$ ) was calculated from the number of cells counted (Q) according to the formula: $N=1 /$ ssf * 1 /asf * $1 /$ hsf * $Q$.

\section{Statistical analysis}

All data are represented as mean + standard error of means (+ SEM). For each parameter normality was tested using a Kolmogorov-Smirnov test. All data were normally distributed. Differences in birth weight, litter size, mortality and cell numbers/densities were tested using a one-way analysis of variance (ANOVA). Body weights over time were tested using a repeated measures ANOVA. All significant effects were analyzed in more detail using post-hoc Bonferroni tests. The accepted level of statistical significance was $p<0.05$ for all analyses. All calculations were done using the Statistical Package for the Social Sciences (SPSS 15.0 software, Chicago, IL, USA). 


\section{Results}

\section{Gestational length, litter size and mortality}

All dams reached full-term pregnancy (data not shown). Neither the sham-operation, nor the FA procedure had an effect on gestational length. Additionally, litter size did not differ between groups (data not shown). There was no significant increase in prenatal mortality due to the SHAM and FA interventions. Figure 1 shows the mortality at birth per litter. There was no mortality in the CVD and CCD group and a low level of mortality in the SHAM (7\%) and FA (13\%) group. Mortality was significantly higher in the SPA (66.8\%), SHAM/SPA (53.7\%) and FA/SPA (23.2\%) groups. Moreover, preconditioning significantly reduced the mortality due to SPA at PO (SPA versus FA/SPA; -65\%; $p<0.05$ )

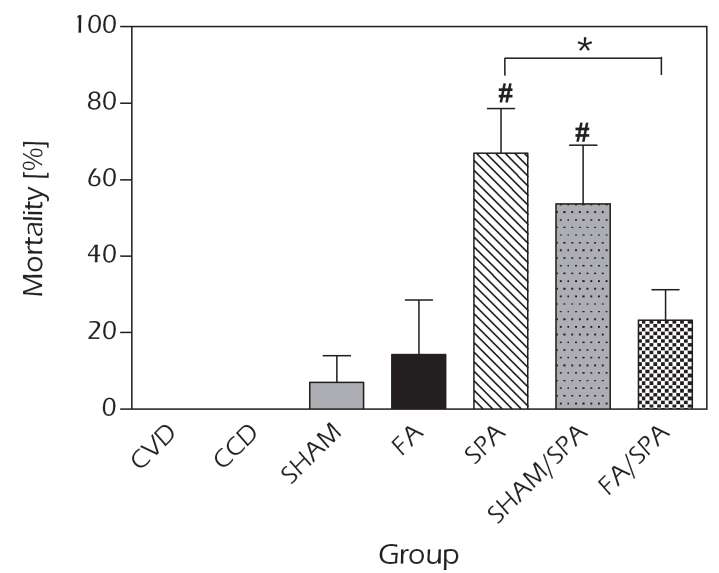

Figure 1: Mortality rates per litter at birth.

SPA and SHAM-SPA significantly increased the mortality at birth. The preconditioning stimulus significantly decreased the mortality as shown by the FA/SPA group. Data are expressed as mean mortality per litter + SEM. Abbreviations: CVD: control vaginally delivery, CCD: control caesarian delivery, SHAM: sham-operated controls, FA: fetal asphyxia, SPA: severe perinatal asphyxia. (One-way ANOVA + post-hoc Bonferroni testing; * $\mathrm{p}<0.05$, \# significantly differs from CVD, CCD, SHAM, FA and FA/SPA)

\section{Somatic growth}

Birth and body weights are presented in figure 2. One-way ANOVA analysis showed an overall effect on birth weight (Figure 2A). Post-hoc Bonferroni demonstrated that both SHAM (-9\%, $p<0.01)$ and FA (-1 1\%; $<<0.01)$ pups had 
significantly lower birth weights than control animals that did not undergo a procedure before birth (CVD, CCD and SPA). Mean body weights were measured at P7 and P14 (Figure 2B). A repeated measured ANOVA showed a significant overall effect of both time $(p<0.001)$ and group $(p<0.001)$. At $P 7$, SHAM/SPA $(p<0.05)$ and FA/SPA $(p<0.05)$ animals were significantly lighter than CVD and CCD animals. At P14, SHAM ( $p<0.05)$, FA ( $p<0.05)$, SHAM/SPA $(p<0.05)$ and $F A / S P A(p<0.05)$ animals were significantly smaller than the CVD, $C C D$ and SPA animals.
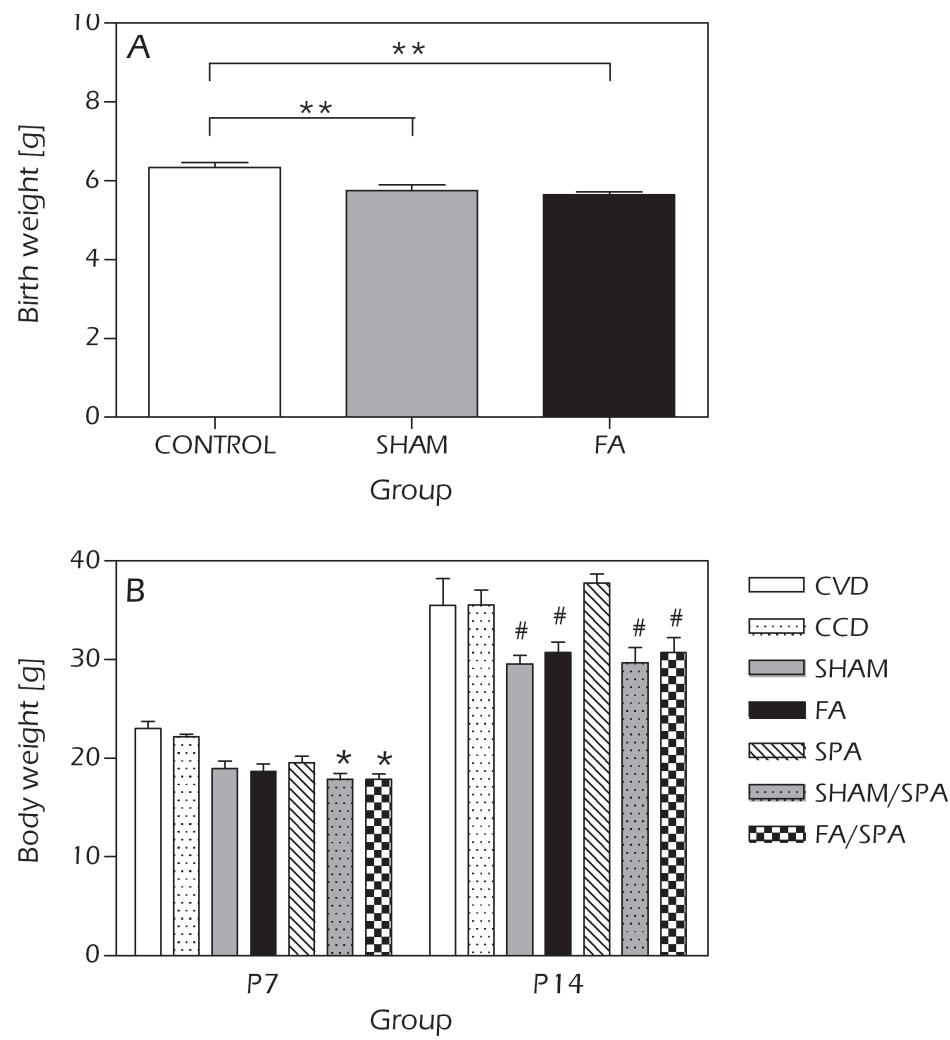

Figure 2: Mean birth and body weights.

(A) Mean birth weights. SHAM and FA animals were significantly lighter at birth in comparison to control animals. (B) At postnatal day 7 (P7), SHAM/SPA and FA/SPA animals were significantly smaller than CVD and CCD animals. AT P14, SHAM, FA, SHAM/SPA and FA/SPA animals had significantly lower body weights than CVD, CCD and SPA animals. Data are expressed as mean+ SEM. Abbreviations: P: postnatal, CVD: control vaginally delivery, CCD: control caesarian section, SHAM: sham-operated control, FA: fetal asphyxia, SPA: severe perinatal asphyxia. (One-way ANOVA + post-hoc Bonferroni; * significantly differs from CVD and CCD ( $p<0.05)$; \# significantly differs from CVD, CCD and SPA $\left.(p<0.05) ;{ }^{* *} p<0.01\right)$ 


\section{Brain weights, ratios and volumes}

Mean brain weights and body-to-brain ratios are given is figures 3A-B. No significant differences were found between any of the groups in brain weight (Figure 3A) and brain-body ratios (Figure 3B). Figures 4A-F depict the volume estimates of the total brain (4A), the lateral ventricles (4B) and the corpus callosum (4C), the striatum (4D), the frontoparietal cortex (4E) and the hippocampus (4F). No significant differences were found between groups for any of these volumes estimates.
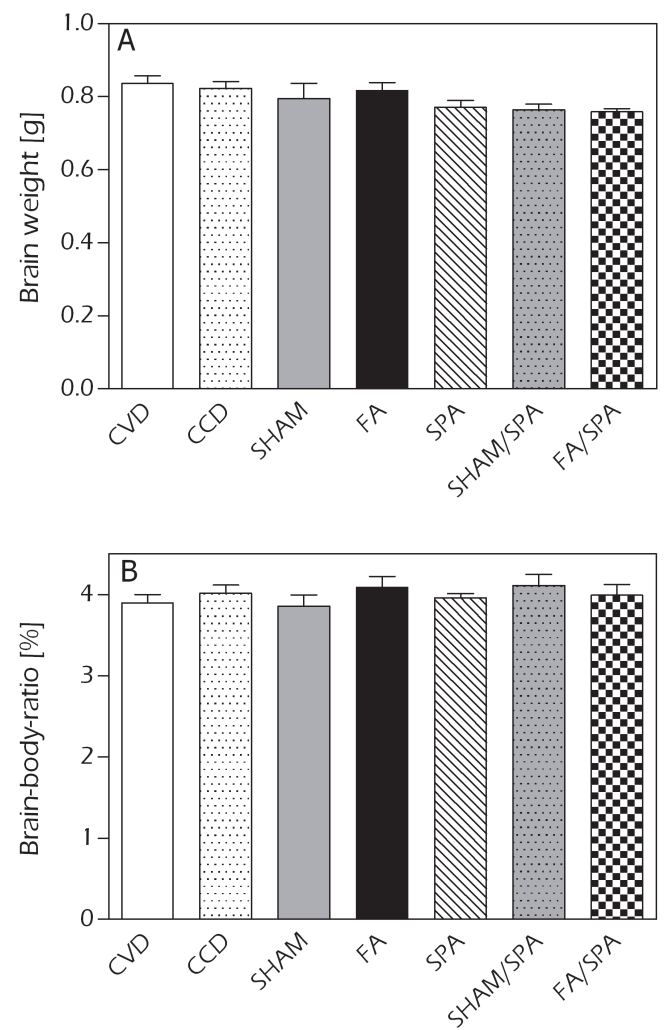

Figure 3: Brain weights and brain-body ratios at $\mathrm{P7}$.

(A) Brain weights at P7. No significant differences were found in brain weights between the groups at P7. (B) No significant differences were found in body-to-brain ratio between the different groups at P7. Data are expressed as mean + SEM. Abbreviations: CVD: control vaginally delivery, CCD: control caesarian delivery, SHAM: sham-operated controls, FA: fetal asphyxia and SPA: severe perinatal asphyxia. 

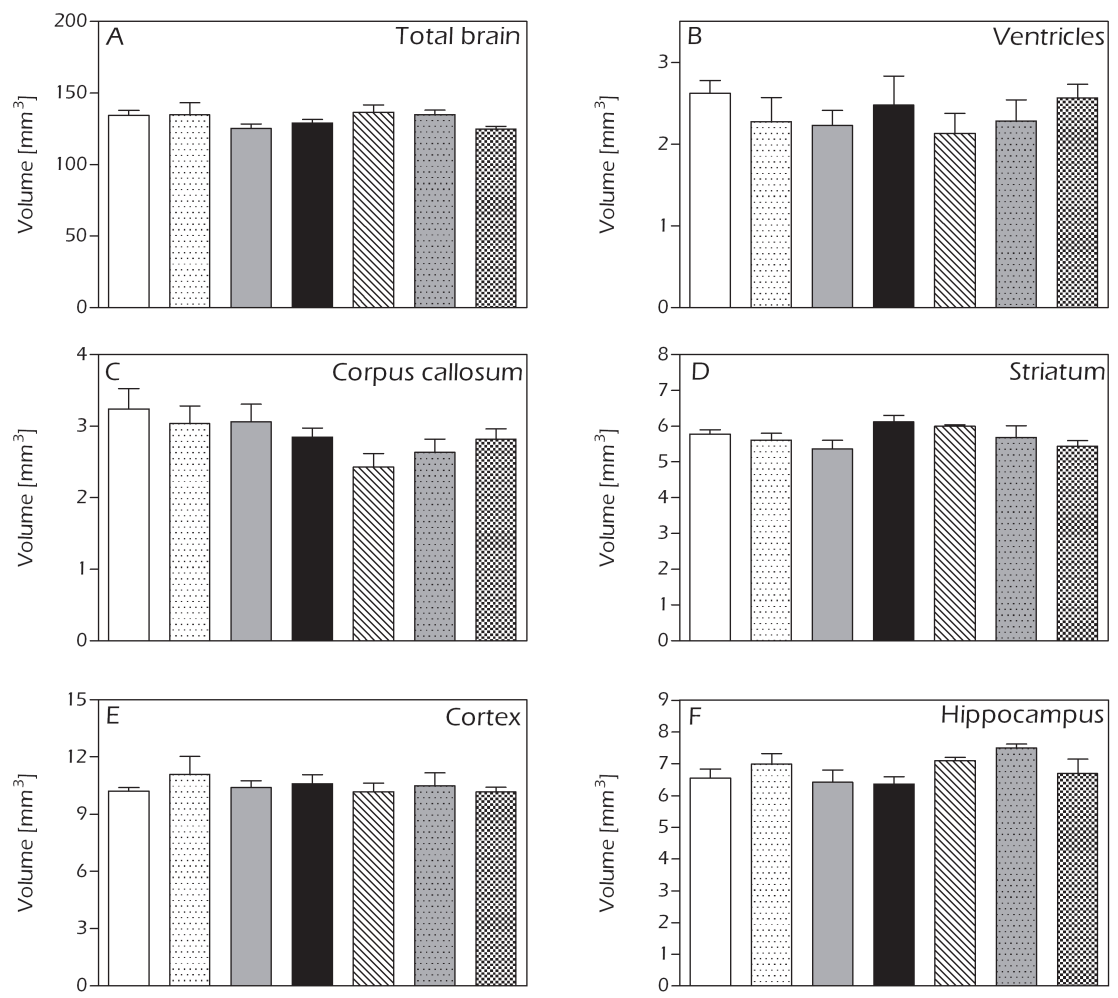

$\square$ CVD $\square$ CCD $\square$ SHAM $\square$ FA SPA

Figure 4: Volume estimates at P7

(A) Volume estimates of the total brain at P7. (B) Volume estimates of the lateral ventricles. (C) Volume estimates of the corpus callosum. (D) Volume estimates of the striatum. (E) Volume estimates of the frontoparietal cortex. (F) Volume estimates of the hippocampus. No sifnificant differences were found between the groups for any of the volume estimates measured. Data are expressed as mean + SEM. Abbreviations: CVD: control vaginally delivery, CCD: control caesarian delivery, SHAM: sham-operated controls, FA: fetal asphyxia and SPA: severe perinatal asphyxia.

\section{TUNEL-positive apoptotic cell death}

The mean number and density of TUNEL-positive cells in the striatum, frontoparietal cortex and hippocampus are shown in figure 5A-F. In the striatum (figure 5A-B), SPA animals showed a significantly higher number and density of TUNEL-positive cells in comparison to the other groups (pos-hoc Bonferroni; 
$p<0.01$ ). In addition, SHAM/SPA animals also significantly differed from CVD animals (post-hoc Bonferroni; $\mathrm{p}<0.05$ ). In the frontoparietal cortex (figure 5CD) and the hippocampus (figure 5E-F), similar results were found. In those two areas, SPA animals showed a significantly increased number and density of TUNEL- positive cells compared to all other groups (post-hoc Bonferroni; $p<0.05)$. The most important findings in this analysis are that preconditioned FA/SPA animals had 1) a lower number and density of TUNEL-positive cells than the non-preconditioned SPA animals, and 2) similar number and density of TUNEL-positive cells than the control groups in all areas investigated.

\section{GFAP-positive astrocytes}

Figures 6A-F show the mean number and mean density of GFAP-positive astrocytes in the striatum, frontoparietal cortex and hippocampus. In the striatum (figure 6A-B), the mean number and density of GFAP-positive astrocytes was significantly higher in the SPA than in the CVD, CCD, SHAM, FA and FA/SPA groups (post-hoc Bonferroni; $p<0.01$ ). SHAM/SPA significantly differed from the CVD, CCD, SHAM, FA and FA/SPA groups as well (post-hoc Bonferroni; $p<0.05$ ). In the frontoparietal cortex (figure $6 C-D$ ) and the hippocampus (figure 6E-F), SPA also significantly increased the number and density of GFAPpositive astrocytes in comparison to all other groups (post-hoc Bonferroni; $p<0.05)$. The main findings, however, are that the preconditioned FA/SPA animals had 1) a significantly lower number and density of GFAP-positive astrocytes than the non-preconditioned SPA animals, and 2) similar number and density of GFAP-positive astrocytes compared to control animals in all three areas investigated.

\section{Discussion}

In this present study, we examined whether mild fetal asphyxia (FA), as a preconditioning stimulus, can protect against the consequences of severe perinatal asphyxia (SPA). The main findings are that FA attenuates the SPA-induced mortality and neuropathology. The preconditioned animals showed significantly less apoptosis and less astrogliosis 7 days after perinatal asphyxia than 

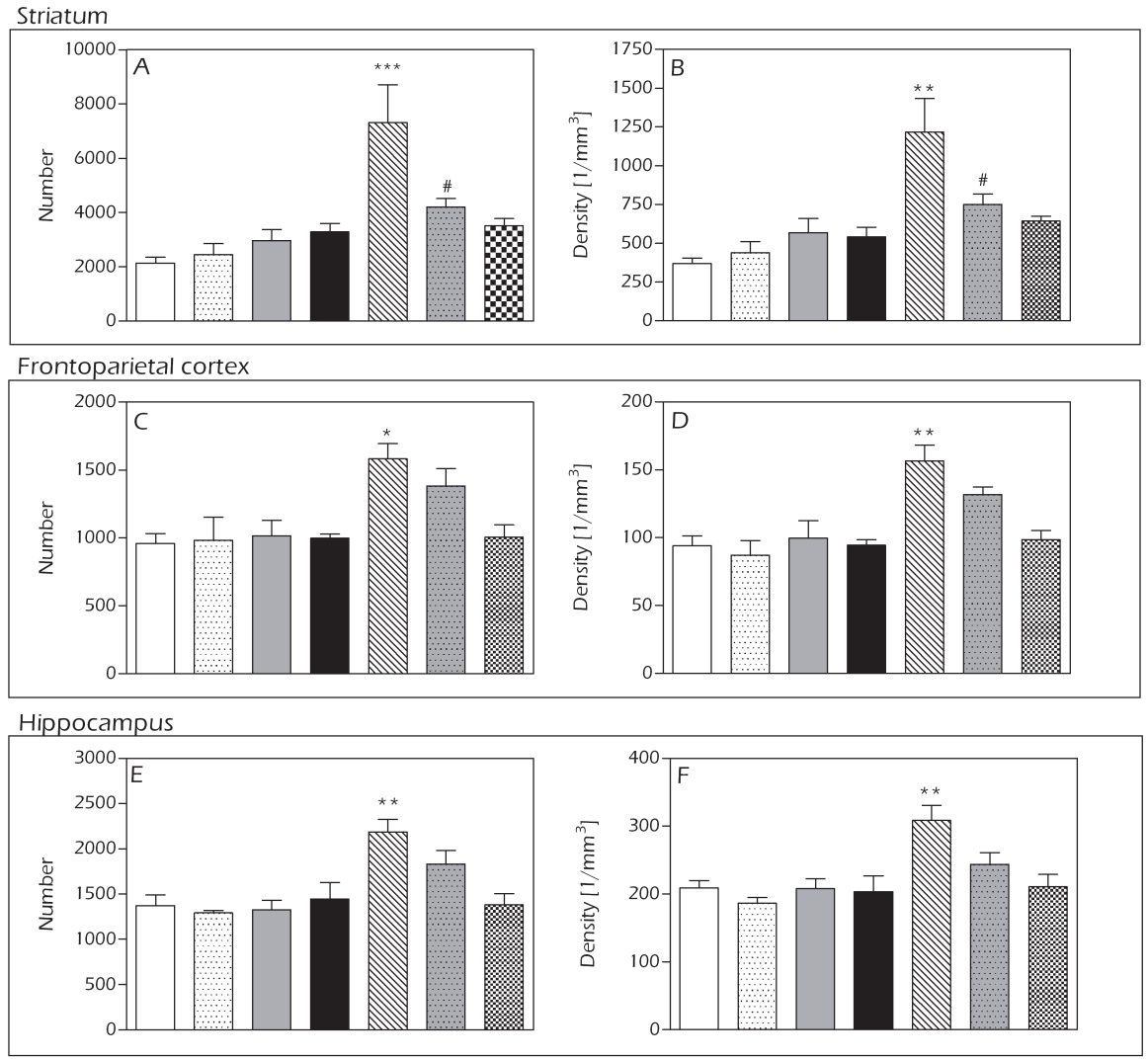

$\square$ CVD CCD SHAM SPA SHAM/SPA FA/SPA

Figure 5: Mean number $(A, C, D)$ and mean density $(B, D, F)$ of TUNEL-positive cells in different brain areas.

(A) The mean number of TUNEL-positive cells in the striatum. (B) The mean density of TUNEL-positive cells in the striatum. (C) The number of TUNEL-positive cells in the frontoparietal cortex. (D) The mean density of TUNEL-positive cells in the frontoparietal cortex. (E) The mean number of TUNEL-positive cells in the hippocampus. (F) The mean density of TUNEL-positive cells in the hippocampus. Data are expressed as mean + SEM. Abbreviations: CVD: control vaginally delivery; CCD: control caesarian section; SHAM: sham-operated controls; FA: fetal asphyxia; SPA: severe perinatal asphyxia. ( ${ }^{*}$ significantly differs from CVD, CCD, SHAM, FA and FA/SPA (post-hoc Bonferroni; $p<0.05$ ); ** significantly differs from CVD, CCD, SHAM, FA and FA/SPA (post-hoc Bonferroni; $p<0.01$ ); *** significantly differs from CVD, CCD, SHAM, FA and FA/SPA (pos-hoc Bonferroni; $p<0.001$ ); \# significantly differs from CVD (post-hoc Bonferroni; $p<0.05$ )) 


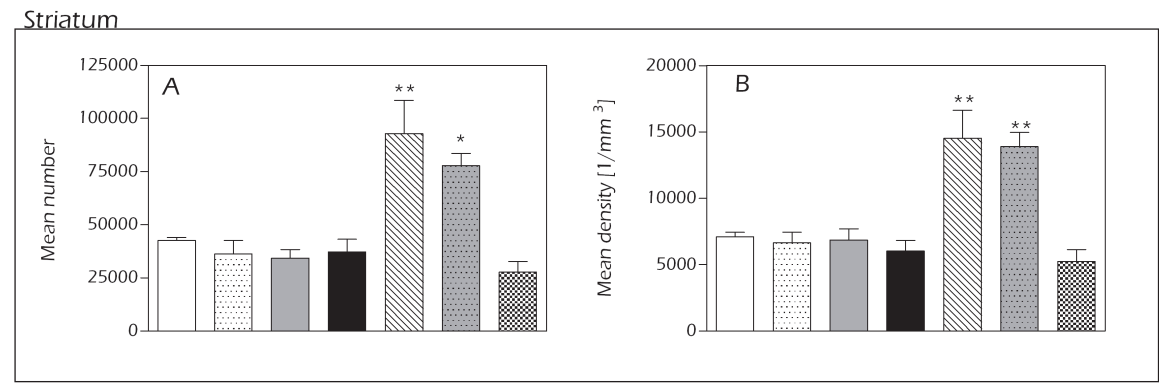

Frontoparietal cortex
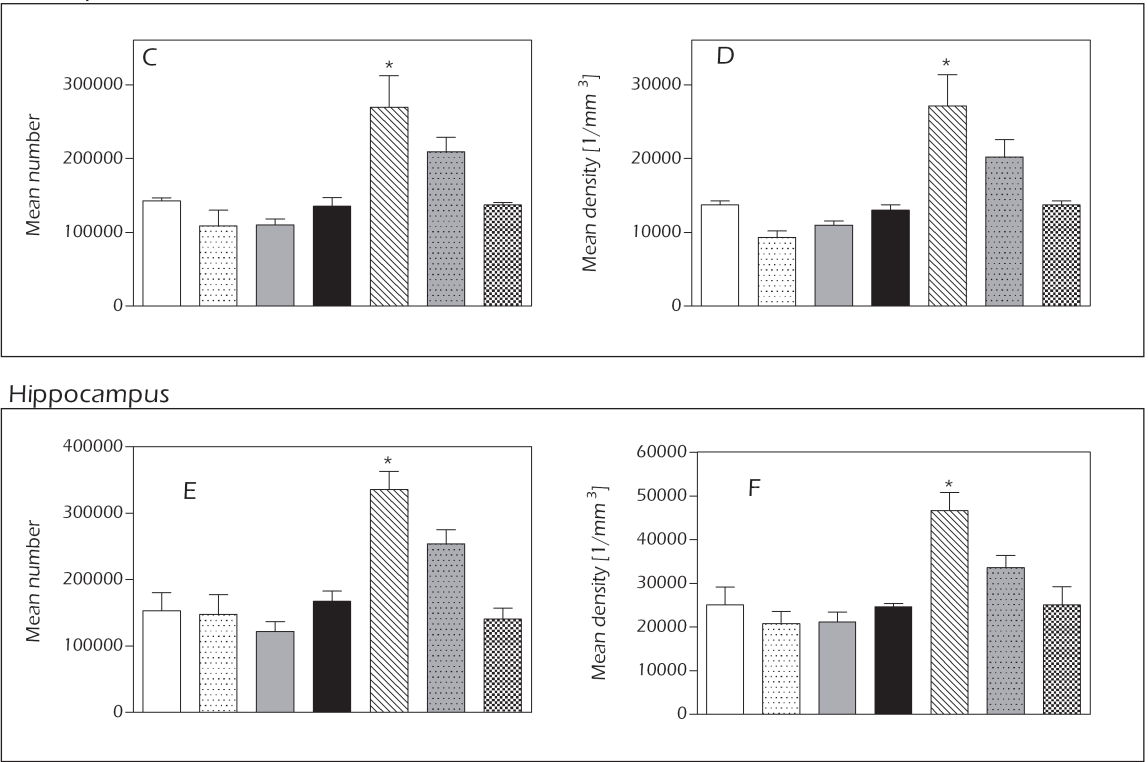

$\square$ CVD

Figure 6: The mean number $(A, C, E)$ and the mean density $(B, D, F)$ of GFAP-positive astrocytes in different areas of the brain.

(A) The mean number of GFAP-positive astrocytes in the striatum. (B) The mean density of GFAP-positive astrocytes in the striatum. (C) The mean number of GFAP-positive astrocytes in the frontoparietal cortex. (D) The mean density of GFAP-positive in the frontoparietal cortex. (E) The mean number of GFAP-positive astrocytes in the hippocampus. (F) The mean density of GFAP-positive astrocytes in the hippocampus. Data are expressed as mean + SEM. Abbreviations: CVD: control vaginally delivery; CCD: control caesarian section; SHAM: sham-operated controls; FA: fetal asphyxia; SPA: severe perinatal asphyxia. (* significantly differs from CVD, CCD, SHAM, FA and FA/SPA (post-hoc Bonferroni; $p<0.05$ ); ** significantly differs from CVD, CCD, SHAM, FA and FA/SPA (posthoc Bonferroni; $p<0.01) 1$ 
the non-preconditioned animals in all areas investigated, i.e. the striatum, the frontoparietal cortex and the hippocampus.

\section{Severe perinatal asphyxia causes a rise in apoptotic cell death and astrogliosis}

It has been demonstrated many times before that the neonatal brain is very susceptible to apoptotic cell death after asphyxia and that apoptosis plays a key role in neonatal asphyctic injury. Corresponding with the current literature, our data show that SPA significantly increased the number and the density of apoptotic cells compared to control animals at P7 in all areas investigated. Van de Berg and colleagues also demonstrated that SPA increases the number of apoptotic cells in the striatum [58]. Similarly, a higher number of apoptotic cells were reported by Dell'Anna and colleagues in the frontal cortex, the striatum and the cerebellum 8 days after SPA [59]. In contrast to our study, they did, however, not find a significant increase in the hippocampus.

The increase in apoptotic cell death after SPA in the striatum, frontoparietal cortex and hippocampus was accompanied by an increase in the number and density of GFAP-positive astrocytes (astrocytic hyperplasia). Similar increases in GFAP-positive cells were reported a few times before in different animal models of developmental asphyxia. Benjelloun and colleagues showed proliferation of astrocytes apparent $24 \mathrm{~h}$ after a left common carotid artery occlusion at P7 [313]. Furthermore, Ezquer and colleagues found an increased GFAP expression at 48h after severe hypoxia in a 7-day-old rat pup [314].

\section{Fetal asphyxia protects against apoptosis and astroglio- sis induced by severe perinatal asphyxia}

The mechanism of neonatal preconditioning still remains unclear. Based on the results of this study, a plausible mechanism might be the prevention of apoptotic cell death. The results show that the preconditioned FA/SPA animals 
had a significantly lower number and density of TUNEL-positive cells in the striatum, frontoparietal cortex and hippocampus. Although the beneficial effect of hypoxic or ischemic preconditioning in the neonatal brain has been reported many times before, we are the first to use a fetal preconditioning stimulus to protect against an asphyctic insult at birth. In another model, Cantagrel et al. already demonstrated that hypoxic-preconditioning protects against apoptosis caused by carotid ligation in a 7-day-old rat pup [126]. Comparable to our results, they found a decreased number of apoptotic cells in the striatum, cortex and hippocampus. The mechanisms explaining this attenuation in apoptotic cell death still have to be elucidated, but it probably involves several pathways. Different neonatal preconditioning models already found a few players that are likely to be involved. Lu et al. demonstrated that NF-KB is critical in the establishment of neuroprotective anti-apoptotic effects [315]. CREB and antiapoptotic genes, like Bcl-2 also seem to play an important role [129, 136, 316]. Others showed that the production of NO and NOS are essential for the induction of tolerance [121, 140, 317].

Another possible mechanism contributing to neonatal neuroprotection might be that the preconditioning stimulus, besides apoptosis, also protects against an astrogliosis reaction. After all, this study shows that the preconditioned FA/SPA animals have a significantly lower number and density of GFAP-positive astrocytes than the non-preconditioned SPA animals. This is the first report showing less astrogliosis in neonatal preconditioning. Astrogliosis is a common neurocellular event in brain pathology as a response to many diseases and/or injury [207, 208]. Its precise cause and function is still not known. For quite some time, it has been thought that astrogliosis was just a response to neuronal death to replace the lost neurons, but recent data suggest that astrogliosis is probably pathological process which can induce detrimental effects. Astrogliosis is associated with inflammatory processes and the production of reactive oxygen species [209-211]. Additional evidence suggests glial tissue can interfere with normal functioning of neuronal circuits by inhibiting regeneration and causing electrical instability [212]. Consequently, the preconditioning stimulus might cause neuroprotection by preventing astrogliosis to contribute to the malfunctioning of the certain brain areas. 


\section{Time window for preconditioning}

In the heart, preconditioning was found to be characterized by relatively short windows of protection. The first protection phase appears immediately after the preconditioning stimulus and only lasts for only 60-120 minutes [318, 319]. A second window of protection extends between $24 \mathrm{~h}$ and $72 \mathrm{~h}$ [320]. In contrast to the relatively short windows of protection in the heart, preconditioning in the brain appears to induce two relatively longer windows of tolerance [321]. In the adult rat brain, the first window, which is called rapid preconditioning, usually peaks $1 \mathrm{~h}$ after the stimulus, while the second window, called delayed preconditioning, is detectable from $24 \mathrm{~h}$, peaks at 3 days and gradually fades away until 7 days after the stimulus $[116,117,321,322]$. Nowadays, almost all preconditioning studies in the neonatal brain use a 24-hour time window between the hypoxic preconditioning stimulus and the actual insult [55, 124-126, 323]. In our study, however, the time frame between the two was much longer. The FA stimulus was applied 4 days before SPA was induced and our data show that neuroprotection was still established. In addition, Xiao and colleagues demonstrated that FA might induce an even longer window of neuroprotection [121]. They showed that mild fetal asphyxia at E17 still protects against carotid artery ligation in a 7-day-old rat pup ( 11 days). Together, this indicates that FA-induced neuroprotection has a relatively long time window and that it probably involves mechanisms of delayed neuroprotection, which requires new protein synthesis and gene expression.

\section{Fetal asphyxia as a preconditioning stimulus}

The results of this study do not show overt morphological changes due to the preconditioning stimulus per se, i.e. 30 minutes of FA. FA did not cause any differences in the number of apoptotic and GFAP-positive cells compared to control animals. Nevertheless, both FA and the sham-procedure had a significant effect on birth and body weight. As stated by the Barker hypothesis, low birth weight infants have a higher risk to develop adult-onset diseases as cardiovascular problems [324]. Neurological problems, like schizophrenia, ADHD, depression and learning problems, have been related to lower birth weights as well [325-330]. Consequently, it is important for the future studies to investigate 
the long-term functional outcome of these low birth weight animals. An additional reason to test the long-term functional outcome is that when FA is applied for a longer period of time, i.e. 75 minutes, clear morphological and behavioral deficits will develop later in life $[152,153]$. Severe FA causes the loss of nigral dopaminergic neurons associated with impaired locomotion at 6 months of age [153]. Additionally, at 19 months of age serotonergic cells were lost in the dorsal raphe nucleus, which was related to increased anxiety-related behavior [152], and severe FA also resulted in the loss of striatal synapses [331].

\section{General conclusion}

In conclusion, this study demonstrates that mild fetal asphyxia can protect against apoptosis and astrogliosis induced by severe perinatal asphyxia. Consequently, mild FA might cause neuroprotection by inducing anti-apoptotic mechanisms and by attenuating astrogliosis. Future studies are, however, necessary to study the long-term functional consequences of this neuroprotective strategy and to evaluate the mechanisms underlying this type of tolerance. Identifying these mechanisms may provide potential targets for future pharmacological intervention. 

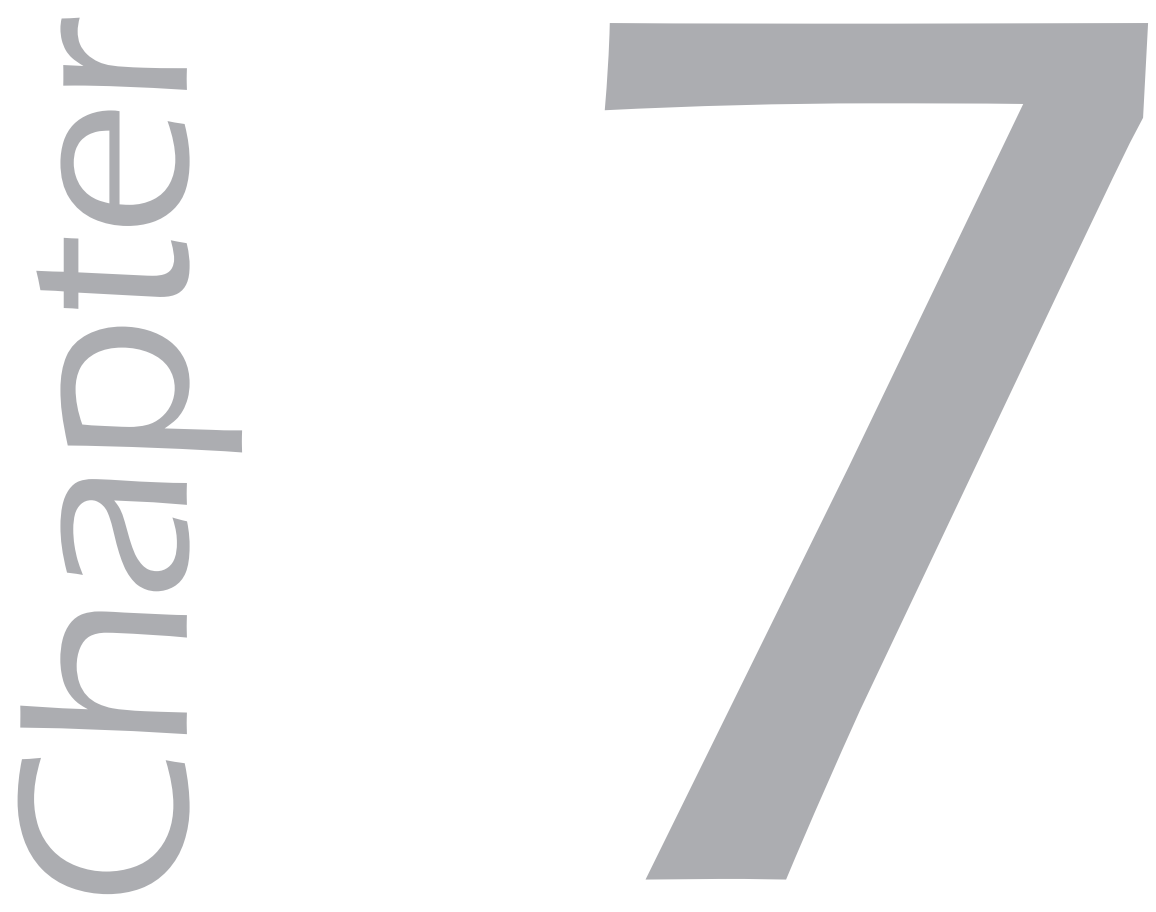

Fetal asphyctic preconditioning protects against perinatal asphyxia-induced behavioral consequences in adulthood

E.Strackx, D.L.A. Van den Hove, J. Prickaerts, L. Zimmermann, H.W.M. Steinbusch, C.E. Blanco, A.W.D. Gavilanes and J.S.H. Vles 


\begin{abstract}
Perinatal asphyxia is one of the major causes of neuronal injury and impaired development in infants. We recently have shown that a brief episode of experimental fetal asphyxia (FA) can provoke an endogenous neuroprotection against subsequent severe perinatal asphyxia (SPA). The long-lasting functional consequences of FA preconditioning are not clear yet. The aim of the study was to determine if FA preconditioning can provide a long-lasting behavioral protection against SPA. FA was induced, as a preconditioning stimulus, by clamping the uterine vasculature for 30 minutes on E17. At birth, SPA was induced by placing the uterine horns in a water bath for 19 minutes. At 6 months of age, functional outcome was assessed using different behavioral tests: the open field for locomotor activity, the elevated zero maze for anxiety-related behavior, the forced swim test for depression-related behavior and the object recognition task for cognition. Data showed that FA preconditioning improved postnatal mortality after SPA. At the age of 6 months, the total distance moved in the open field and elevated zero maze was significantly less in the SPA group compared to the control groups. In addition, cognitive performance in the object recognition task was impaired in the SPA offspring compared to the control groups. Most importantly, FA preconditioning was able to preserve both locomotor activity and cognition function. In conclusion, FA preconditioning induces a long-lasting, functional protection against SPA. Therefore, this model seems to offer good opportunities for the identification and characterization of the underlying mechanisms of preconditioning.
\end{abstract}




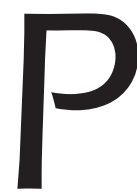

erinatal asphyxia is one of the major causes of neonatal mortality and morbidity, contributing to neuronal injury and impaired development in infants. It is frequently associated with long-term deficits, like motor and cognitive problems [6, 161, 259-261, 284]. Except for post-asphyctic hypothermia, there is still a lack of effective therapeutic strategies to treat post-asphyctic encephalopathy [312]. Interestingly, we have recently shown that a brief episode of experimental fetal asphyxia can provoke endogenous neuroprotective mechanisms against a subsequent more severe asphyctic insult at birth $([332,333]$ in press). This phenomenon is called "hypoxic-ischemic preconditioning" or "asphyctic preconditioning" [332]. The molecular mechanisms underlying hypoxic-ischemic or asphyctic preconditioning have not been fully elucidated yet.

For the immature brain, a preconditioning animal model has been developed in which exposure to $8 \%$ oxygen 1 day before unilateral carotid artery occlusion in a 7-day-old rat pup significantly reduces the infarct volume [124]. Unilateral carotid artery occlusion merely causes one-sided focal brain damage, which does not reproduce the most common pathological type of global asphyctic encephalopathy in the human neonate. Furthermore, the model does not take into account the unique physiological mechanisms specific to the time of birth that may also play an important role in the development of post-asphyctic brain injury. For these reasons, the present study used a new rat model, combining global fetal asphyxia (FA), as a preconditioning stimulus, with severe global asphyxia (severe perinatal asphyxia; SPA) at birth.

In addition, it is important to note that practically all reports on hypoxic-ischemic preconditioning focus on the short-term, histological outcome. Shortterm, histological neuroprotection was already established in our model. The FA preconditioning stimulus seems to protect against apoptotic cell death caused by SPA [333]. However, the long-term, functional consequences of FA preconditioning are not clear yet. Therefore, the aim of this study was to determine if this new rat model, e.g. FA preconditioning, can also provide a longlasting protection at a behavioral level. For this purpose, the uterine vasculature was clamped for 30 minutes on embryonic day 17 (E17), as was previously done by Cai and colleagues [130]. On postnatal day 0 (PO), the uterine horns still containing the fetuses were placed in a water bath for 19 minutes. At 6 months of age, functional outcome was assessed using different behavioral 
tests. The open field task was chosen to study spontaneous locomotion, since it is known that asphyxia during development results, depending on the time point investigated and the model used, in either hypo or hyperactivity [166, $197,274]$. It has also been reported that perinatal asphyxia can induce anxietyrelated behavior, especially in adult animals. Weitzdoerfer et al. showed that SPA leads to increased anxiety-related behavior in 2-year-old animals in the elevated plus maze [103]. For that reason, the elevated zero was performed. The forced swim task was selected to study the response to stress/depressionrelated behavior, because Boksa et al. reported that 10 or 15 minutes of perinatal anoxia causes increased immobility over 6 trials of the forced swim task [334]. The last test performed was the object recognition task to test memory/cognitive function, since a few reports showed that perinatal asphyxia is associated with mild defects in both learning and spatial and non-spatial memory, tested in the Morris water maze, multiple T-maze and novel object recognition $[57,97,101,102]$. Finally, basal and stress-induced plasma corticosterone levels were measured to test the responsiveness of the hypothalamo-pituitaryadrenal (HPA) axis to explain potential differences in affective behavior.

\section{Materials and methods}

\section{Animals}

All experiments were approved by Animal Ethics Board of Maastricht University on animal welfare according to Dutch governmental regulations. All efforts were made to minimize the pain and stress levels experienced by the animals and to minimize the number of rats necessary to produce reliable scientific data. Sprague-Dawley rats, obtained from Charles River (Germany), were kept under standard laboratory conditions with food and water given ad libitum, $21 \pm 2^{\circ} \mathrm{C}$ environment temperature, a $12 \mathrm{~h}$ light/dark schedule (lights on at 07:00 h) and background noise provided by radio. Exclusively male offspring were used within this study, because both morphological and behavioral evidence show a differential vulnerability to a birth insult in males versus females [191, 192]. 


\section{Preconditioning model and study groups}

The new rat model for FA preconditioning is as a combination of two previously well described models. A model for FA is used as a first hit at embryonic day 17 (E 17), while a model for SPA is used as a second hit at E22 [57, 130, 152, $153,335]$. Seven groups of 10-12 offspring were used (Table 1; 82 offspring in total). To control for effects of the Caesarian section procedure in stead of vaginal delivery at PO the CCD group was used and to control for the anesthetics and operation at E17, the SHAM group was added.

Pregnant rats were randomly assigned to an experimental group. No more than two male pups per litter per condition were examined to prevent litter effects. Therefore, in total 70 pregnant dams were used to obtain 82 offspring. Breeding was done by the authors themselves and pregnancy was determined by the observation of vaginal plugs at EO.

Table 1: An overview of the experimental groups

\begin{tabular}{lcccc}
\multicolumn{1}{c}{ Experimental group } & N & Intervention time & Abbreviation \\
\hline Control vaginal delivery & 8 & $/$ & CVD \\
\hline Control Caesarian delilvery & 7 & E21-22 & CCD \\
\hline SHAM-operated & 8 & E17 & SHAM \\
\hline Fetal asphyxia & 8 & E17 & FA \\
\hline Severe perinatal asphyxia & 8 & E21-22 & SPA \\
\hline SHAM + perinatal asphyxia & 7 & E17 + E21-22 & SHAM/SPA \\
\hline Fetal asphyxia + perinatal asphyxia & 8 & E17 + E21-22 & FA/SPA \\
\hline Abbreviations: N: number: E:Embryonic day & & &
\end{tabular}

Abbreviations: N: number; E:Embryonic day

\section{Fetal asphyxia procedure}

At E 17, pregnant rats were anesthetized by isoflurane (induction 4\%, maintenance $1,5 \%$ ). To prevent hypothermia, all procedures were done within a controlled environment $\left(37^{\circ} \mathrm{C}, 60-80 \%\right.$ humidity, normal gas values). Rats were subjected to a midline laparotomy to expose both uterine horns. FA was achieved by completely clamping both the uterine and the ovarian arteries with removable clamps. In the mean time, the uterine horns were kept wet, by rinsing them with a saline solution. After 30 minutes, the clamps were removed 
to allow reperfusion. The uterine horns were placed back intra-abdominally and the abdominal wall was repaired. SHAM-operated rats underwent the same procedure except for the clamping.

\section{Severe perinatal asphyxia procedure}

At E22, after the first pup was born vaginally, pregnant rats were euthanized by decapitation and rapidly hysterectomized. The uterine horns containing the fetuses were detached. One uterine horn was placed in a water bath, calibrated at $37^{\circ} \mathrm{C}$, for exactly 19 minutes (starting from cutting off the blood circulation of the uterus to the moment the pups were taken from the water bath). Afterwards, the uterine horn was dissected quickly and the pups were removed, cleaned with medical swipes and stimulated manually to breathe in a neonatal closed incubator $\left(37^{\circ} \mathrm{C}, 60-80 \%\right.$ humidity, room air). Subsequently, they were left to recover for approximately 60 minutes in the same controlled environment. The umbilical cords were ligated and cut to separate the pups from there placentas. Pups were randomly cross-fostered to surrogate dams, which had given birth normally the same day. Each surrogate mother received 14 pups, i.e. 2 pups from each intervention group. Body weights of the pups were measured at the following time points: PO, P7, P14, P21, 2 months, 3 months and 6 months.

\section{Behavioral experiments}

The different behavioral tasks were performed from least stressful to most stressful in the following order: 1. 'Object recognition task', 2. 'Open field task', 3. 'Elevated zero maze task' and 4. 'Forced swim task'. All tests were started at the age of 6 months and executed within the 4 consecutive weeks.

\section{Object recognition task}

To measure cognition, the object recognition task (ORT) was performed as described in detail before [336]. In brief, the rat was placed in a circular arena, $83 \mathrm{~cm}$ in diameter, with a $40 \mathrm{~cm}$-high enclosure which is half transparent and opaque. Two objects were placed in the arena in a symmetrical position. Dur- 
ing the first week, animals were allowed to adapt to the arena and procedure before the first trial. A testing session was comprised of two trials, each with a duration of 3 minutes. The animal was always placed facing the wall in the middle front segment of the arena. During the first trial (T1), the animal was exposed to two identical objects, while during the second trial (T2) it was presented to two dissimilar objects, i.e. a familiar one and a new one. Four different sets of objects were used, each object being available in triplicate. All combinations were used in a balanced manner to reduce potential biases due to object preference. This procedure was done with different delay-intervals, namely 1, 4 and 24 hours. The time spent exploring each object during $\mathrm{T} 1$ and $\mathrm{T} 2$ was scored manually on a personal computer. Exploration is defined as touching or directing the nose to the object at a distance of no more than $2 \mathrm{~cm}$. Sitting on the object is not considered as exploratory behavior. To avoid the presence of olfactory clues, the arena and the object were cleaned with ethanol. The discrimination index ( $d 2$ ), as a measure of cognitive performance, was calculated as follows: time spent exploring the new object minus the time spent exploring the old object divided by the total time spent exploring both objects during $\mathrm{T} 2$.

\section{Open field task}

To study spontaneous locomotor behavior, the animals were tested in an open field as described previously [153]. This test was conducted by placing the rat in the centre of a $50 \times 50 \mathrm{~cm}$ plexiglas, square box with a $30 \mathrm{~cm}$-high enclosure. The movements were recorded for 60 minutes with a film-camera attached to a computer running the Ethovision ${ }^{\mathrm{TM}}$ program (Noldus Equipment, Wageningen, The Netherlands). The total distance moved and the mean velocity while moving were measured.

\section{Elevated zero maze task}

To study anxiety-related behavior, the elevated zero maze (EZM) was used as previously described [337]. The EZM is a circular alley $1100 \mathrm{~cm}$ diameter; $10 \mathrm{~cm}$ path width), which is elevated $20 \mathrm{~cm}$ above the floor. It is divided into four parts, namely 2 open and 2 closed parts. The rats were placed in one of the open parts (facing the closed part) and were allowed to explore the maze for 5 min- 
utes. Movements were scored automatically using an infrared video camera and a computer system (Ethovision Pro, Noldus, Wageningen, The Nederlands). Parameters determined were time spent in each part of the maze and total distance travelled.

\section{Forced swim task}

To score depression-related behavior, the forced swim task (FST) was performed $[338,339]$. Animals were placed into a plexiglass cylinder $140 \mathrm{~cm}$ tall; $17 \mathrm{~cm}$ diameter) filled with water $\left(30 \mathrm{~cm}\right.$ high) maintained at $23-25^{\circ} \mathrm{C}$. The movements of the rats were automatically scored with a computerized system (Ethovision Pro, Noldus, Wageningen, The Nederlands) during a 10-minute session under low light conditions. The behavioral variables scored were: immobility, mobility and strong mobility (immobility/mobility threshold: 11.5 and mobility/strong mobility threshold: 17). Immobility reflects no movement at all and/or minor movements necessary to keep the nose above the water Mobility represents swimming and strong mobility reflects 'escape behavior' like climbing against the walls or diving.

\section{Measurement of the corticosterone response}

To test the responsiveness of the hypothalamo-pituitary-adrenal (HPA) axis, stress-induced plasma corticosterone secretion was measured. This was done one week after behavioral testing. For this purpose, rats were stressed by placing them in a small mice cage filled with $500 \mathrm{ml}$ water of $23-25^{\circ} \mathrm{C}$ for 20 minutes (always between 10 a.m. and 1 p.m.). Blood samples were collected via saphenous vein puncture immediately before the stressor to measure basal levels and immediately after the stressor. The rat was then returned to the home cage and left undisturbed for 40 minutes after which a third blood sample was taken to measure the recovery. Blood samples were kept cold on ice and centrifuged at 5000rpm for 10 minutes at $4^{\circ} \mathrm{C}$ and the plasma was frozen down at $-80^{\circ} \mathrm{C}$ for subsequent use. Corticosterone was measured directly on $1 \mathrm{ml}$ dried dichloromethane and extracted for radioimmunoassay using corticosterone ${ }^{-125}$. The radioimmunological reaction was performed overnight at $4^{\circ} \mathrm{C}$ after which a secondary antibody system was used to separate bound and unbound steroid as previously described [340]. 


\section{Statistics}

All data are represented as mean + standard error of means (+ SEM). For each parameter normality was tested using a Kolmogorov-Smirnov test. Differences in birth weight, litter size, mortality, open field parameters and forced swim task parameters were tested using a one-way analysis of variance (ANOVA). Body weights over time and ORT were analyzed using a repeated measures ANOVA. All significant effects were analyzed in more detail using post-hoc Bonferroni tests. Since the time spent in the elevated zero maze was not normally distributed, a non-parametric Kruskal-Wallis test was used. The accepted level of statistical significance was $p<0.05$ for all analyses. All calculations were done using the Statistical Package for the Social Sciences (SPSS 15.0 software, Chicago, IL, USA).

\section{Results}

\section{Gestational length, litter size and mortality}

All pregnant dams delivered at E22. Neither the sham-operation, nor the FA procedure had an effect on gestational length. Additionally, litter size did not differ between groups. There was no significant increase in prenatal mortality due to the SHAM and FA interventions. Figure 1 shows the perinatal mortality per litter assessed 60 minutes after birth. One-way ANOVA analysis showed a significant effect of group on mortality $(F(6,70)=15.41 ; p<0.0001)$. At P0, there was no mortality in the CVD, CCD and SHAM group and a low level of mortality $(4.7 \%)$ in the FA group. Post-hoc bonferroni testing showed that mortality was significantly higher in the SPA (66.7\%), SHAM/SPA (55.8\%) and FA/SPA (33.4\%) groups. Moreover, preconditioning significantly reduced the mortality due to SPA at PO (SPA versus FA/SPA; -50\%; $p<0.01$ ). In contrast, SHAM-SPA mortality did not significantly differ from either SPA or FA-SPA. In addition, mortality was also assessed in at P7, P14 and P21, but no significant differences were found between the groups. Remarkably, two SPA animals died around the age of 5 months, while no other animals died after P21. 


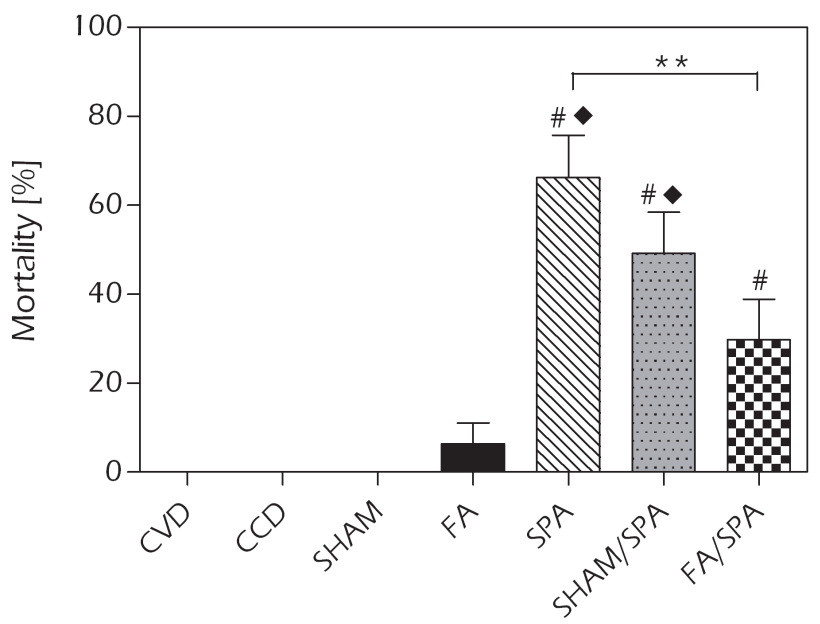

Figure 1: The mortality rates per litter at birth.

At birth, no CVD, CCD or SHAM animals died. There was a significant increase in mortality in the SHAM/SPA, the FA/SPA and the SPA group. Most importantly, FA/SPA group showed a lower mortality than the SPA group. Data are expressed as mean mortality per litter + SEM. ( $n=7$ litters for CVD, CCD, SHAM and FA group and $n=14$ litters for the SPA, SHAM-SPA and FA-SPA group; One-way ANOVA + post-hoc Bonferroni; ** $p<0.01$; \# significantly differs from CVD, CCD and SHAM; significantly differs from FA)

\section{Somatic growth}

Birth weights are depicted in figure 2A. Only birth weight of the control groups (CVD+CCD), the FA group and the SHAM group are given in this figure, since SPA cannot have any effect on the birth weight yet. We found an overall effect on birth weight (One-way ANOVA; F(2,230)=98.87; $p<0,001)$. Post-hoc Bonferroni analysis demonstrated that the body weights of pups that underwent a sham-operation (SHAM and SHAM/SPA; -14\%) or FA procedure (FA and FA/SPA; $-14 \%$ ) were significantly lower than pups that did not undergo any intervention at E17 (CVD, CCD and SPA).

Body weights were also measured throughout the course of the study at P7, P14, P21, 2 months, 3 months and 6 months. Mean body weights at the different time points are given in figure 2B. At P7, no significant differences were found between groups. At P14, FA/SPA and SHAM/SPA animals had signifi- 
cantly lower body weights than CVD animals. At P21, post-hoc analysis showed that FA, SHAM, SHAM/SPA and FA/SPA animals had lower body weights compared to CVD, CCD and SPA animals. At that time point, SPA animals did not differ from controls. At 2 months of age, SHAM, FA, SHAM/SPA and FA/SPA still differed from CVD, CCD and SPA animals, while at 3 months only the SHAM/SPA and the FA/SPA animals differed from the CVD. At 6 months of age, in contrary, SPA animals were significantly smaller than CVD, $C C D$, FA and FA-SPA animals. As shown in figure $2 C$, the FA animals differentiated into two distinct groups at the age of 6 months. One group that was significantly heavier than the CVD $(+12 \%$; $p<0.05)$ group and the other group that was significantly smaller than the CVD group (-15\%; $p<0.01)$.
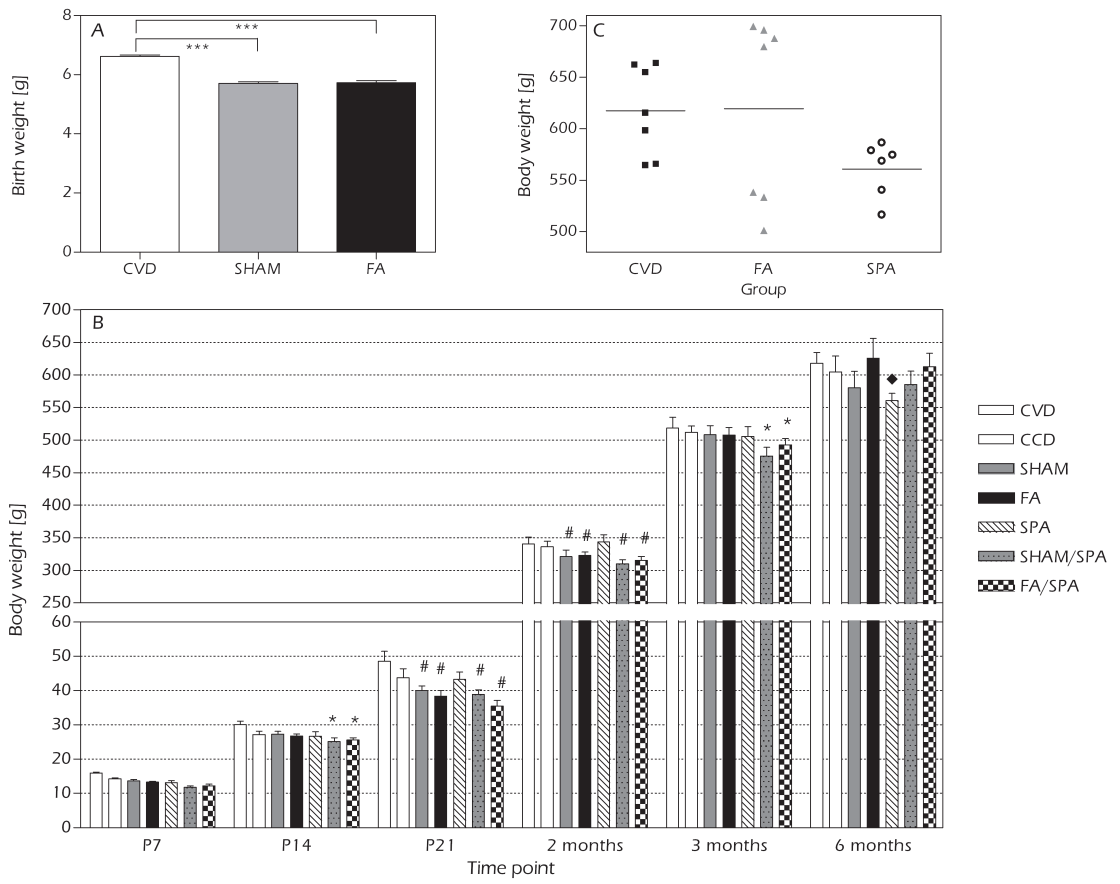

Figure 2: Birth and body weights

(A) Birth weights of the control, SHAM-operated and fetal asphyxia pups. (One-way ANOVA + post-hoc Bonferroni; ${ }^{* *} P<0.001$ ) (B) Body weights of the animals at P7, P14, P21, 2 months, 3 months and 6 months of age. (Two-way ANOVA + post-hoc Bonferroni; \# differs significantly from CVD, CCD and SPA; * differs significantly from the CVD group; significantly differs from CVD, CCD, FA and FA-SPA) (C) The body weights of the CVD, FA and SPA animals at 6 months of age. FA animals differentiated into two different groups. A group that was significantly smaller than the control animals and a group that was significantly heavier than the control animals. Data are expressed as mean + SEM. 


\section{Brain weights}

In figure $3 A-B$ the brain weights and brain-body ratios at 6 months of age are shown. One-way ANOVA analysis showed a significant effect of group on brain weights $(F(6,38)=3.37 ; p<0.01)$. Brains of SPA animals weight significantly less than the other groups (CVD, CCD, SHAM, FA, SHAM-SPA and FA-SPA; Posthoc Bonferroni; $p<0.05$; figure $3 A$ ). When corrected for body weight, however, no significant differences were found (figure 3B).
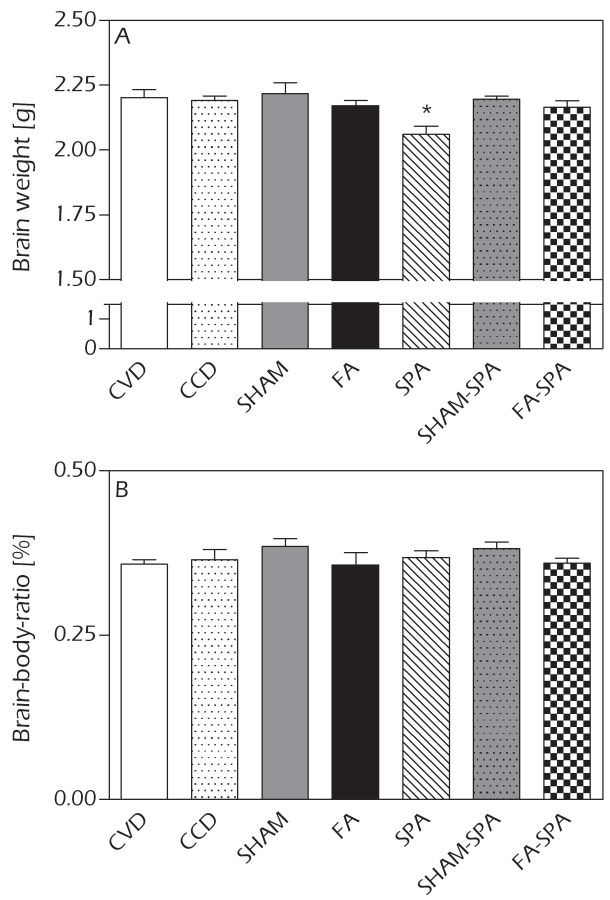

Figure 3: Brain weights

(A) Brain weights of the animals at 6 months of age. SPA brains were significantly smaller than the brains of the other groups. (B) Brain-body ratios of the animals at 6 months of age. When corrected for body weight, no significant differences were found in brain weights. Data are expressed as mean + SEM. (Two-way ANOVA + post-hoc Bonferroni; * differs significantly from the CVD, CCD, SHAM, FA, SHAM-SPA and FA-SPA group; $p<0.05$ ) 


\section{Behavioral testing}

\section{Open field (OF)}

Figure 4 depicts the results of the OF, expressed as total distance moved [in $\mathrm{cm}$ ] and mean velocity while moving [in $\mathrm{cm} / \mathrm{s}]$. There was an overall effect on the total distance moved (One-way ANOVA; F(6,72)=3.45; $p<0.01$; Figure 4A). Post-hoc Bonferroni testing showed that the SPA group and the SHAM/SPA significantly differed from the CVD, CCD, SHAM, FA and FA/SPA group. The preconditioned group (FA/SPA) performed equal to the control groups. There were no significant differences between the groups in mean velocity (Figure 4B).
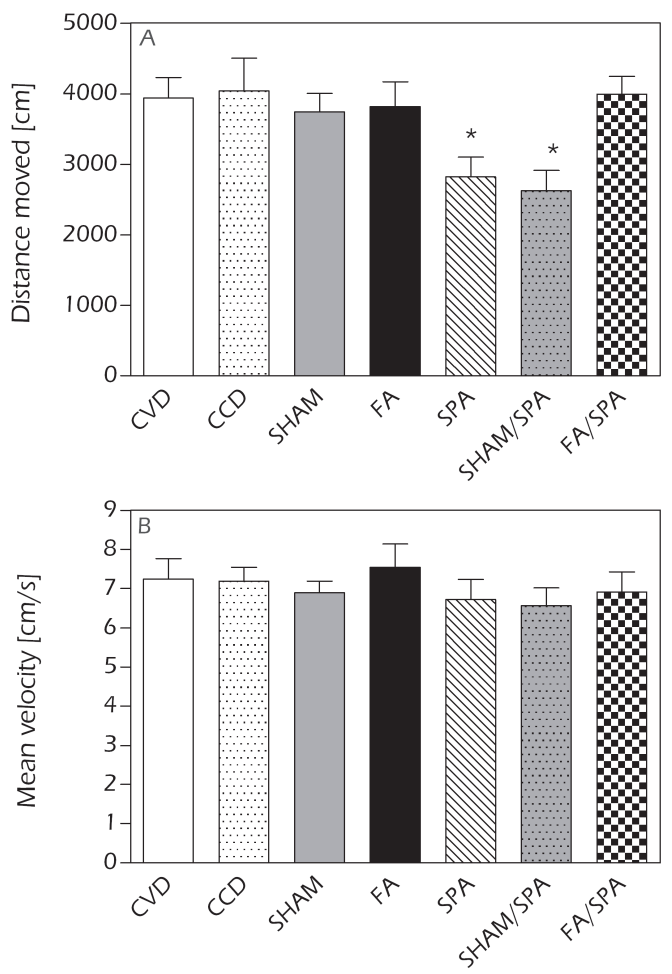

Figure 4: Open field

(A) The mean distance moved [in cm]. The SPA and the SHAM/SPA groups moved significantly less than the other groups (One-way ANOVA; $p<0.01$ ) (B) The mean velocity while moving [in $\mathrm{cm} / \mathrm{s}$ ]. There were no significant differences in velocity between the groups. (One-way ANOVA + Post-hoc Bonferroni test; * differs significantly from CVD, CCD, SHAM, FA and FA/SPA groups) 


\section{Elevated zero maze (EZM)}

The results of the EZM are shown in figure 5. Data are expressed as time spent in the open and closed arms [in s] and as total distance moved in the open arms, the closed arms and the total arena [in $\mathrm{cm}$ ]. No significant differences were found in the time spent in either the open or closed arms. There was, however, a significant effect of group on the total distance moved (Two-way ANOVA; group $x$ zone; $F(12,213)=4.02 ; p<0.001)$. Post-hoc Bonferroni test showed that SPA significantly lowered the distance moved in the total arena compared to CVD, CCD, SHAM, FA and FA/SPA.
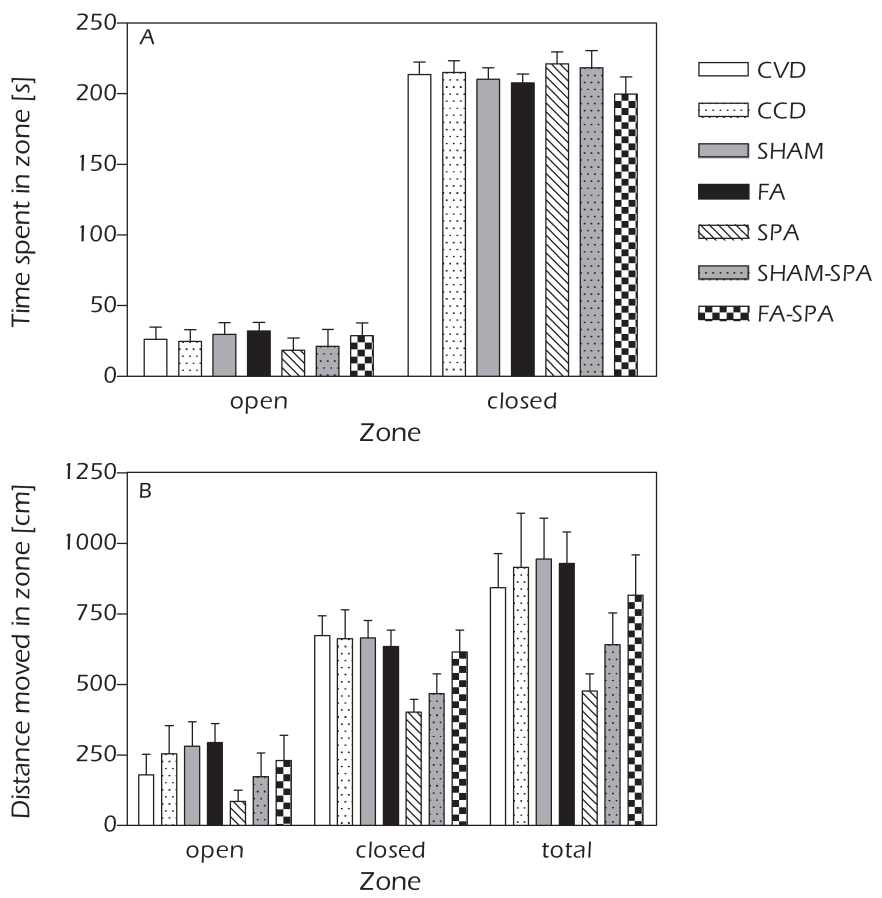

Figure 5: Elevated zero maze

(A) The time spent [in s] in the open en the closed arms of the elevated zero maze. No significant differences were found between the groups. (B) The total distant moved [in cm] in the open and closed arms, as well as the total arena of the elevated zero maze. No significant differences were found between the groups in the distance moved in the open and the closed arms. For the total distance moved, however, the SPA group moved significantly less than the CVD, CCD, SHAM, FA and FA/SPA groups (One-way ANOVA + Post-hoc Bonferroni test; * differs significantly from CVD, CCD, SHAM, FA and FA/SPA groups; $p<0.01$ ) 


\section{Forced swim task (FST)}

The mobility was measured during the forced swim task, expressed as time spent immobile, mobile and, when shown, strong mobile. No significant differences were found between the groups for any of the parameter measured (data not shown).

\section{Object recognition task (ORT)}

Figure 6 represents the results of the ORT. The animals were tested at 3 different time-intervals, i.e. 1, 4 and 24 hours, and the relative discrimination index (d2) was used as a measure of object memory. The repeated measures ANOVA showed an overall significant effect of group $(F(6,215)=7.61 ; p<0.001)$ and time $(F(2,215)=41.27 ; p<0.001)$. This difference could be completely ascribed to the SHAM/SPA and SPA groups. Post-hoc Bonferroni testing showed that the SPA and SHAM/SPA group had a significantly smaller $d 2$ over time than the all other groups ( $P<0.01$ for all comparisons). The preconditioned group (FA/SPA) performed equal to the control groups. In addition, a one-way ANOVA analysis showed a significant overall effect of group at the 1-hour interval $(p<0.01)$ and at the 4-hour interval $(p<0.05)$. At the 1-hour interval, SPA and SHAM/SPA animals had a significant smaller $d 2$ than the other animals (resp. $p<0.01$ and $p<0.05$ for all comparisons; post-hoc Bonferroni test). Similarly, at the 4-hour interval the SPA and SHAM/SPA groups scored significantly lower than the other groups ( $p<0.05$ for all comparisons; post-hoc Bonferroni). No significant effects were seen at the 24-hour interval. No significant differences were found between the SPA and the SHAM/SPA group at any of the intervals. Furthermore, additional analysis of location preference (left of right side of the arena) and object preference showed no differences between groups /data not shown).

\section{Corticosterone response}

Table 2 shows the results of the corticosterone measurements at 6 months of age. Corticosterone levels were measured under basal, stress and recovery condition. Two-way ANOVA analysis showed a significant effect of condition $|F| 2$, 218 ) 150.8 ; $p<0.001$ ) on the level of corticosterone. For all 7 groups, the 20 
minute stress period significantly increased the level of plasma corticosterone in comparison to basal level and after the 40 minute recovery period the corticosterone level dropped back to basal level. Moreover, no significant differences were found between the groups under any of the conditions.

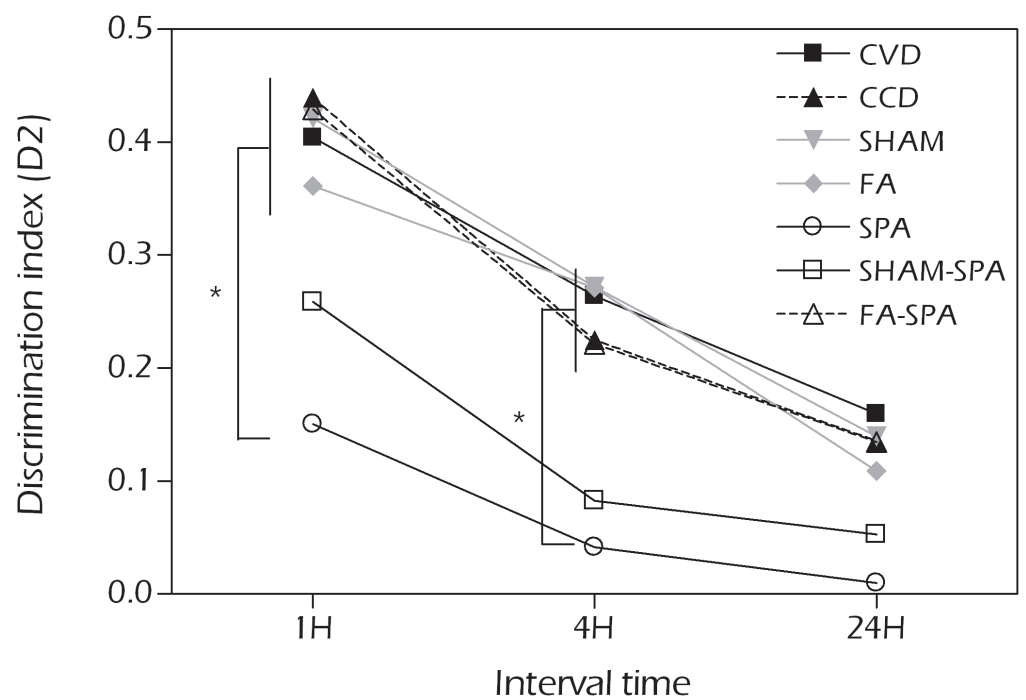

Figure 6: Object recognition task

The relative discrimination index (d2) at different time-intervals, i.e. 1, 4 and 24 hours, during the object recognition task. The $d 2$ of the SPA and the SHAM/SPA group was significantly lower over time than the other groups (repeated measures ANOVA; $p<0.001+$ post-hoc Bonferroni; * $p<0.01$ ).

Table 2: Corticosterone measurements

\begin{tabular}{lcccccc} 
& \multicolumn{2}{c}{ BASAL } & \multicolumn{2}{c}{ STRESS } & \multicolumn{2}{c}{ RECOVERY } \\
& MEAN & SEM & MEAN & SEM & MEAN & SEM \\
\hline CVD & 180.33 & 22.73 & 368.09 & 21.66 & 186.26 & 19.81 \\
\hline CCD & 174.63 & 22.31 & 291.34 & 38.80 & 209.20 & 49.04 \\
\hline SHAM & 145.39 & 16.73 & 264.94 & 46.12 & 133.36 & 32.32 \\
\hline FA & 149.82 & 22.14 & 304.57 & 42.62 & 134.28 & 25.19 \\
\hline SPA & 150.53 & 29.21 & 289.86 & 41.97 & 180.18 & 33.90 \\
\hline SHAM/SPA & 169.08 & 60.53 & 361.67 & 55.83 & 183.69 & 72.91 \\
\hline FA/SPA & 149.70 & 12.38 & 366.30 & 14.42 & 146.38 & 14.94
\end{tabular}

No significant differences were found between the groups under any of the conditions. Abbreviations: CVD: control vaginal delivery, CCD: control caesarean delivery, SHAM: sham-operated, FA: fetal asphyxia, SPA: severe perinatal asphyxia. $\mathrm{N}=12$ for each group. (One-way ANOVA) 


\section{Discussion}

Almost all experimental reports on neuroprotective strategies, like pharmacological interventions, hypothermia and preconditioning against post-asphyctic brain injury focus on the immediate, histological outcome [124-126]. Neuroprotection is typically established by quantifying the reduction in infarct volume or the degree of cell death a few days after the insult [124-126]. Nevertheless, post-asphyctic injury is a dynamic process extending up to weeks or even months after the initial insult $[58,152,154]$, which does not necessarily result in extensive or explicit brain damage on a morphological level $[89,155]$. In some cases, it only results in subtle deficits in brain functioning or behavior. These might be reasons why some clinical neuroprotective strategies failed in a long-term setting, despite good protective results in short-term, morphological studies [158]. Some of the protective strategies might just delay the injury rather than providing a lasting protection and histological preservation does not necessarily mean that neuronal functioning is preserved $[156,157]$. Shortterm, histological protection was already established in our model. The FA preconditioning stimulus seemed to protect against early cell death. FA/SPA animals showed significant less apoptotic cells in the striatum and prefrontal cortex than the SPA animals 7days after the insult [[333] in press]. The aim of this study was to determine whether FA preconditioning, can also provide a long-lasting (6 months) protection at a behavioral level.

\section{Why a novel model for asphyctic preconditioning?}

In this present study, we aimed to validate a novel model of FA preconditioning. At E 17, the uterine vasculature was clamped for 30 minutes as a preconditioning stimulus, followed by severe perinatal asphyxia 4 days later. The preconditioning stimulus at E17 was chosen, because it was already established to work by Cai et al [130].

This model has several advantages over the existing models. Both insults are global events that are associated with both systemic metabolic and hemody- 
namic changes that might influence the outcome of the fetus. These changes mimic the global, pathophysiological conditions of asphyxia before and during birth. Furthermore, unique physiological mechanisms specific to the time of birth, like hormonal changes, might play an important role in the development of asphyctic encephalopathy. There are also major physiological differences, like energy consumption, between a near-term fetus and 1-day-old animal. Overall, the fetus is more resistant to hypoxia than the neonate [141-143]. For these reasons, it is particularly important to study the multifactorial effects of asphyxia and preconditioning during the processes of pregnancy and delivery. The insults used in this study, therefore, reflect the clinical situation more closely than commonly used stroke models that are induced in a 7-day-old rat pup. Stroke models merely cause one-sided focal brain damage, which does not simulate the most common pathological type of global asphyctic encephalopathy in the human neonate [168]. However, the brain of a rat fetus at birth is more immature than that of a human infant. With regard to synaptogenesis and myelinization, the rat brain only resembles the human equivalent at approximately 10 days after birth [145-148]. Nonetheless, premature neonates more often suffer from perinatal asphyxia and hypoxic-ischemic encephalopathy than term infants $[149,151]$, making these time point of interference relevant as well.

Of note, fetal asphyctic preconditioning will never be clinically applicable. Nevertheless, this new model might be a good approach to unravel the underlying mechanisms of neuroprotection during fetal or early neonatal development. These unknown mechanisms and new targets might be different in comparison to the existing models because of a few reasons. First, this new model uses global insults in stead of the commonly used Rice-Vannucci model, which induces only focal brain damage. Therefore, different inducers and effectors might come into play due to the distinct nature of the insults. Next, the second insult takes place during the process of birth. Since most asphyxia most commonly occurs during birth and birth is characterized by unique physiological mechanisms, it might be important to study the effects under the exact same conditions. 


\section{The post-injury effects of fetal asphyctic preconditioning}

\section{Mortality}

The first observation was a profound reduction in immediate perinatal mortality in the preconditioned animals compared to the non-preconditioned animals. Since the FA preconditioning stimulus affects the fetus globally, it is plausible that the stimulus provides whole-body protection. The lower mortality rates may, therefore, be caused by the protective effects on different organs and systems, like the cardiovascular system [341, 342].

\section{Somatic growth}

The second important observation was the somatic growth, measured as birth and body weights of the offspring. At birth, FA and SHAM animals were significantly smaller than control animals. Since the weight reduction was the approximately the same for both groups, this is most likely due to the intervention performed at E17, i.e. anesthesia and manipulation of the uterus. Post-operative effects on the feeding behavior of the mothers can be ruled out, since the SHAM-operated and FA-operated dams gained a similar amount of weight during pregnancy as the unoperated control dams (data not shown). These differences in body weight disappeared at P7 and reappeared at P14, which possibly reflects an unsuccessful attempt trying to make up the growth arrear. Finally, body weight gradually equalized over time. At 6 months of age, half of the FA animals were even heavier than control animals, which will be discussed in the next paragraph.

\section{Behavioral effect of severe perinatal asphyxia}

The third important observation was that SPA caused significant deficits in both spontaneous locomotor activity and cognition. SPA animals moved significantly less than the control animals in both the open field and the elevated zero maze. Additionally, SPA animals performed worse in the object recognition task. Previous studies had already demonstrated that different asphyctic insults in the developing fetus can cause a number of functional impairments, like slower eye opening and righting reflex, changes in locomotor and sensorimotor ac- 
tivity and memory deficits in the neonatal, juvenile and adult periods [89, 98, $100,343,344]$. In addition, Kiss et al. showed in a similar model that perinatal asphyxia causes a marked delay in neurobehavioral development [345]. Reflexes like negative geotaxis, sensory, righting, grasp gait and auditory startle reflexes were delayed for 1-4 days. Most studies, however, used the Rice-Vannucci stroke model, which results in a different injury pattern than the SPA model, for which only a handful of studies have been published. In one of these studies, Boksa et al. showed that 10, 15 or 20 minutes of birth asphyxia caused mild deficits in spatial learning in the Morris water maze at 4 months of age [101]. In the same model, Van de Berg et al. and Weitzdoerfer et al. also described long-term spatial memory impairments in aging rats 18 months and 2 years, respectively) also using Morris water maze testing [57, 102]. In addition, Simola et al. also showed an impairment in non-spatial memory due to a 20 minute perinatal anoxia using the novel object memory [97].

With respect to locomotor activity, this study showed that SPA decreased the distance moved in the open field, while the velocity was unaffected. The distance moved in these animals was also affected in the elevated zero maze. This contradiction between distance moved and velocity can be explained by the fact that the velocity of the animals was only measured while the animal was actually moving. It can therefore be explained by the fact that SPA animals were showed less spontaneous activity (i.e. sitting still more) or moved less of the time as compared to the other animals. This might not only reflect an activity component, but motivational component might be involved as well. Because of the stressful circumstances, this motivational component might be the reason why no differences were found in mobility during the forced swim task Moreover the forced swim task also tests learned helplessness. Our open field results confirm the current literature on neonatal asphyxia. Lower basal activity in the open field and a reduced stereotyped behavior in 6 or 8-month-old animals were reported before [153, 346]. Loidl et al. also found hypoactivity during open field testing in 5-month-old animals exposed to perinatal asphyxia [166]. In contrast, some other studies, mostly inducing hypoxia, report hyperactivity. For example, Cai and colleagues demonstrated that 30 minutes of fetal asphyxia at E17 increased locomotor activity on postnatal days 13 and 15 [93]. A similar increase in locomotor activity was seen between postnatal days 12 and 18 in a bilateral carotid artery occlusion model at P4 [197]. In both studies 
this hyperactivity seemed transient, since it was no longer present after 3 weeks. Furthermore, 19-20 minutes SPA caused a decrease in locomotion, while a milder form (15-16 minutes) caused an increase in locomotion. These inconsistencies may be explained by the type of insult, the severity of the insult, or the timing, i.e. developmental stage of the brain. It is important to note that, like these studies, we did not find any behavioral changes at an earlier time point, i.e. 3 months (pilot data; data not published).

In this study, no significant differences were found on anxiety-related behavior as tested during the elevated zero maze. This is in contrast to previous studies. For example, Weitzdoerfer et al. found increased anxiety-related behavior in the elevated plus maze in 2-year-old perinatal asphyctic animals [103]. Venerosi et al. showed that also other aspects of emotional behavior are affected as well after perinatal asphyxia [347]. They found higher ultrasound production in male asphyctic offspring at P13 and a more ultrasound emission during training session of the fear conditioning task in adult rats. They did, however, not find significant differences in the task itself. A possible explanation for this discrepancy with our results might be that, in comparison to the other studies, 6 months is too early to detect effects on anxiety-related behavior. Moreover, no differences were found in depression-related behavior during the forced swim task. The absence of changes in emotional behavior, like anxiety and depression-related behavior nicely corresponds with the absence of changes in basal and stress-induced corticosterone levels, showing a normal responsiveness of the HPA-axis. This is in accordance with the report of Venerosi et al., which showed a normal physiological response to stress, as measured by corticosterone levels, in the same SPA model [346].

\section{The protective effects of fetal asphyctic preconditioning}

The fourth and most important observation of this study was that the preconditioning stimulus was able to preserve spontaneous locomotor activity as well as cognition in the 6-month-old animals subjected to SPA. In contrast to the SPA animals, FA/SPA animals performed comparable to control animals in the open field, elevated zero maze and object recognition tasks. Similar results have already been reported, though using different preconditioning stimuli and insults. Zhao et al. showed that isoflurane preconditioning improved the motor 
coordination after ischemia in 1-month-old rats [123]. However, isoflurane preconditioning was not able to improve spatial memory performance [343]. Hypoxic preconditioning, in contrast, did improve both sensimotor and spatial/cognitive functions up to 8 weeks after ischemia as shown by Gustavsson et al. [128]. The current study is, however, the first to look at the long-term functional consequences in a preconditioning study with global asphyctic insults. Revealing the mechanisms of neuroprotection was beyond the scope of this study. This will be addressed in the near future.

It is important to note that the SHAM-operation per se caused some protective effects as well: a small decrease in mortality and a minor amelioration of learning/memory. However, these differences between the SPA and SHAM/SPA group were not statistically significant. Concerning the mortality rates, the SHAM-SPA group did also not significantly differ from the FA-SPA group, meaning that part of the beneficial effect is probably caused by the SHAM-procedure. This might be the result of a brief exposure to the inhalation anesthetic, isoflurane. As reviewed by Wang et al., isoflurane can protect the brain against both global and focal hypoxia and/or ischemia [348]. This holds true only for the mortality data, regarding the behavioral data, there was a significant difference between SHAM-SPA and the FA-SPA animals in the open field and the object recognition task. In these test, SHAM-SPA animals behaved almost identical to SPA animals. In summary, the anesthesia might have an important beneficial effect on the mortality rates, while it seems to be the mild fetal asphyxia itself that has important beneficial effects on a behavioral level.

\section{The effects of the preconditioning stimulus per se}

Our study revealed no evidence that the preconditioning stimulus itself, i.e. the 30-minute FA insult, caused any functional changes. The SHAM and FA animals scored exactly the same as the control animals in all behavioral tests performed. In spite of this, SHAM and FA animals had significantly lower birth weights than control animals. These differences in body weight were still apparent after 3 weeks and 2 months. According the Barker hypothesis, lower birth weights, which often reflect adverse intrauterine influences, are associated with a higher risk of adult-onset diseases, like cardiovascular problems [324]. Lower birth weights have been implicated as well as a risk factor for 
schizophrenia, attention deficit/hyperactivity disorder, depression and learning deficits [325-330]. It is important to remark that, based on body weights, FA animals differentiated into two groups at 6 months of age. Half of the animals are heavier than the control animals. This might have important implications concerning the Barker hypothesis. Low birth weights are often followed by growth spurt, often leading to higher than normal body weights in the long run. About a possible link between a growth spurt later in life and neurological dysfunction nothing is published yet. Nonetheless, in this study the weight differences did not seem to have evident functional neurological consequences, though further research is necessary. No significant differences were found between both groups of FA animals for any of the behavioral test. Maybe the 6 month time point is too early to already see behavioral differences since the FA body weights only start to differentiate now.

It is also important to note that no significant differences were found between the CVD and the CCD group, showing that the caesarian section procedure per se had no effects. The CCD group was added to this experiment, because some reports suggest that a C-section might cause a low grade of fetal distress or brain hypoxia during the first $24 \mathrm{~h}$ of life, as shown by altered respiratory rates, activity levels, plasma catecholamines, glucocorticoids and a rise in brain lactate in both rats and humans [349, 350]. In addition, it was also shown that caesarian section causes long lasting effects on dopamine levels and metabolism, dopamine receptor binding, as well as in dopamine-related behavior in adult rats in comparison with vaginal birth [349, 351-354]. Nevertheless, in this study no effects were found of C-section per se on locomotion, anxiety and depression-related behavior or cognition.

\section{Concluding remarks}

FA preconditioning induces a long-lasting functional protection against SPA. This model, therefore, seems to offer good opportunities for the identification and characterization of the underlying mechanisms of preconditioning. Understanding these mechanisms can give us new insights into its clinical potential by providing new targets for future therapies. 

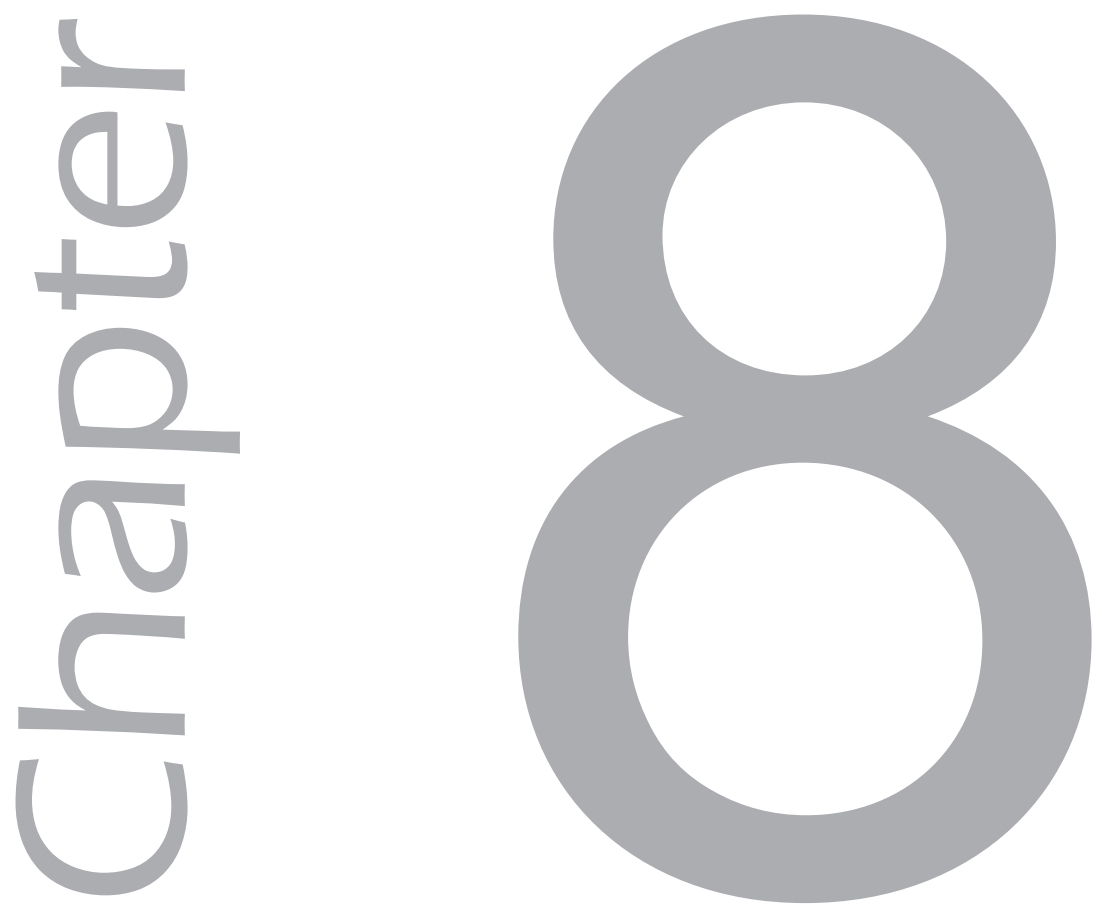

General discussion

E.Strackx 


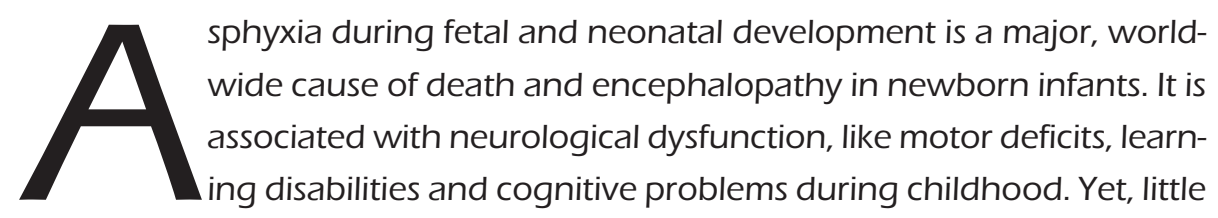
is known about its long-term functional consequences during early adulthood and aging. This is the first main issue that was addressed in this thesis. Chapters 2 to 4 aimed at investigating the long-term behavioral and morphological consequences of severe fetal asphyxia.

Additionally, almost no effective therapeutic strategies are available so far to threat infants with hypoxic-ischemic encephalopathy. Nevertheless, one of the most exciting and promising concepts put forward during the last two decades is the concept of hypoxic-ischemic preconditioning. This is the second main issue addressed in this thesis. Chapters 5 to 7 aimed at developing and validating a new rat model for fetal asphyctic preconditioning. The main results concerning these two topics are summarized below.

\section{The long term effects of severe fetal asphyxia:}

\section{A summary}

In the first part of this thesis, we revealed that severe fetal asphyxia has several long lasting behavioral and morphological consequences.

First, we showed that severe fetal asphyxia can produce long-term impairments in the nigrostriatal dopaminergic system and dopamine-mediated locomotion in 6-month-old rats [153]. Fetal asphyctic rats showed lower basal and amphetamine-induced locomotor activity during open field testing. These behavioral changes were associated with a diminished number of dopaminergic neurons in the substantia nigra pars compacta, a lower tyrosine hydroxylaseimmunoreactivity in the striatum and nigrostriatal astrogliosis. In addition, severe fetal asphyxia caused white matter loss and ventriculomegaly. Second, we demonstrated that severe fetal asphyxia leads to long-term deficits in the raphe serotonergic system and anxiety-related behavior in middle aged rats, i.e. at 19 months of age [152]. We found a decline in the number of serotonergic cells in the dorsal raphe nucleus, combined with higher serotonin-immunore- 
activity. These morphological changes were accompanied by an increase in anxiety-related behavior. Third, severe fetal asphyxia also caused long-term changes in synaptic numbers and densities in the same 19-month-old rats. They showed a significant decrease in the number and density of presynaptic boutons in the striatum, whereas an increase in presynaptic bouton number was found in the 5 th layer of the frontal cortex.

In summary, our observations show that severe asphyxia during pregnancy can cause long-lasting consequences in young adult and middle-aged animals. Since part of these consequences are age-related and only present later in life, fetal asphyxia might induce accelerated aging. This concept was already put forward in the first three chapters of this thesis. In addition, these changes might be of great importance in understanding the pathophysiology of different neurological disorders.

\section{Mild fetal asphyxia as a preconditioning stimulus:}

\section{A summary}

The second part of this thesis concentrates on the development and validation of a new rat model for fetal asphyctic preconditioning. In this new model we used a mild fetal asphyxia insult as a preconditioning stimulus.

First, our findings indicate that mild fetal asphyxia causes a short-term morphological neuroprotection against severe perinatal asphyxia four days later. Preconditioning significantly decreased the number and density of apoptotic cells in the striatum, frontoparietal cortex and hippocampus at postnatal day 8 in comparison to animals without the preconditioning stimulus. Moreover, preconditioned animals had less astrogliosis in response to severe perinatal asphyxia when compared non-preconditioned animals. Second, we illustrated that mild fetal asphyxia also provokes a long-term functional protection against severe perinatal asphyxia-induced behavioral consequences. Preconditioned animals had a better locomotor function and memory performance at the age of 6 months than non-preconditioned animals. 
Summarized, our data show that mild fetal asphyxia, as a preconditioning stimulus, can provide both a short and long-lasting morphological and functional neuroprotection against severe perinatal asphyxia. Therefore, this model seems to offer good opportunities for further identifying and characterizing the underlying mechanisms of asphyctic tolerance.

\section{Mild versus severe fetal asphyxia}

Nowadays it is widely accepted that a noxious stimulus, like hypoxia-ischemia or asphyxia, can cause a wide spectrum of responses ranging from detrimental via no effect at all to even beneficial [5]. Beyond a certain threshold, asphyxia can cause extensive brain injury by for example inducing apoptotic cell death, whereas below that threshold favorable effects like tolerance are induced. This phenomenon was clearly demonstrated in thesis. When fetal asphyxia was applied for 75 minutes, harmful morphological and behavioral consequences became apparent. Like summarized above, animals subjected to a 75-minute fetal asphyctic insult showed a loss in nigral dopaminergic and raphe serotonergic neurons associated with motor and anxiety-related behavioral changes later in life $[152,153]$. When that same insult was applied for a shorter period of time, i.e. 30 minutes, the response appeared to be protective. As shown in this thesis, a 30-minute fetal asphyctic insult caused neuroprotection against severe perinatal asphyxia-induced cell death, astrogliosis and behavioral changes. In addition, the preconditioning stimulus per se did not seem to cause any adverse effects. No short-term morphological or longterm behavioral changes could be observed due to mild fetal asphyxia using this model. The boundaries, however, between the beneficial and detrimental response still have to be determined.

\section{Methodological considerations}

\section{Animal model}

A major stipulation an experimental animal model must fulfill is to mimic the clinical situation as close as possible. The best known and most widely used 
animal model for neonatal hypoxic-ischemic encephalopathy is the Rice-Vannucci model [168]. The model is based on the Levine preparation in the adult rat. In a 7-day-old postnatal rat pup unilateral common carotid artery occlusion is performed followed by systemic hypoxia (8\% oxygen). Although this model has proven very useful to study perinatal brain damage, it strongly deviates from the clinical picture. This Rice-Vannucci model causes merely one-sided focal brain damage, which does not mimic global encephalopathy most often seen in the human neonate. The animal models we used in this thesis, in contrast, are global events that are associated with both systemic metabolic and hemodynamic changes that may influence the outcome of the fetus. In this way, these models better simulate the pathophysiological conditions before and during birth. This was already thoroughly discussed in chapter 1 of this thesis.

\section{Rodent brain development}

The rat brain at birth is more immature than that of the human newborn at term. With regard to synaptogenesis and myelination, the rat brain only resembles the human equivalent at approximately 10 days after birth [145-148]. For that reason, most reports use the Rice-Vannucci model which induces stroke at postnatal days 7-10 [168]. In our experiments, however, asphyxia was induced at either embryonic day 17 or postnatal day 0 . Therefore, our global asphyxia models might more closely reflect the situation in the premature infant. Nonetheless, premature neonates more often suffer from perinatal asphyxia and hypoxic-ischemic encephalopathy than term infants, making these time points of interference as relevant $[149,151]$.

\section{Apoptotic cell death}

The method used to detect apoptotic cell death in this thesis was a TUNEL staining. The TUNEL method detects nuclear DNA fragmentation in cells. Although fragmentation is a pattern typical of apoptosis, it can also occur, to a lesser extent, in necrotic cells. As a consequence, TUNEL can sometime also stain necrotic cells. Because each stained cell was analyzed for apoptotic traits like nuclear fragmentation and a pyknotic nucleus with a Hoechst staining, we assume that the majority of counted cells were apoptotic. In addition, it is cur- 
rently thought that delayed cell death may be apoptotic and/or necrotic and that both forms of cell death may not be mutually exclusive. Hybrid cells can be seen that display morphological features of both types. This has been described as the 'apoptosis-necrosis continuum' [61, 87]. Comparison with adult animal models, however, reveals that apoptosis is more prevalent in the immature brain. Especially the immature cerebral cortex and the basal ganglia contain higher numbers of apoptotic cells.

\section{Implications: Severe fetal asphyxia as an early life risk}

\section{factor for adult disease?}

Most important developmental processes in the brain occur during the prenatal and early postnatal period, when cells are born, connections are made and the brain is myelinized. Different insults can interfere with normal brain development and possible consequences might not be expressed until later in life. Infant or childhood events play an important role in adult disease etiology. This concept is translated into the 'developmental origins of health and disease' hypothesis (DOHaD) [355-357]. Possibly an adverse intrauterine environment can 'reprogram the fetus' in order to survive and this long lasting reprogramming might have adverse consequences in adulthood. Associations have been found between low birth weight, as a marker for adverse intrauterine exposures, and ischemic heart disease, hypertension, obesity and insulin resistance [324]. However, recently, this DOHaD hypothesis has also received attention in relation to neurological and neurodegenerative diseases like depression, Alzheimer's disease or Parkinson's disease [325, 358-362].

Although direct implications for the human fetus cannot be correctly extrapolated yet, this thesis shows that severe fetal asphyxia can cause subtle but longlasting changes in the brain. These changes may be relevant for better understanding the pathogenesis of different adult neurological disorders [166, 180]. Severe fetal asphyxia might be a contributing factor to neurological diseases by making the brain more prone to neurodegeneration or other detrimental processes later in life. Loss of neurons due to early disruptions in brain development (e.g. severe fetal asphyxia; first hit) may produce a long-term vulnerability to a second or third hit (e.g. normal aging or other risk factors) for the 
development of neurological disorders. In chapter 2, for example, we demonstrated the loss of dopaminergic neurons related to a decrease in locomotor activity. It seems plausible that severe fetal asphyxia could accelerate the onset and progression of age-related loss of dopaminergic neurons, enhancing the vulnerability to develop parkinsonian symptoms [223]. Another example is given in chapter 3 . In that chapter, we showed that severe fetal asphyxia causes age-related changes in the raphe serotonergic system associated with increased anxiety-related behavior. Again, it might be possible that fetal asphyxia could be a risk factor for the progression or aggravation of the age-related loss of serotonergic neurons, thereby increasing the susceptibility to develop mood disorders later in life. All the assumptions made above await further research though.

Taken together, severe fetal asphyxia might be a significant risk factor for both the development and the progression of adult diseases. This is an area of investigation that is still unexplored, but may be very relevant for the development of interventions and preventive strategies.

\section{Preconditioning: From animal experiments to clinical practice}

Our knowledge about neonatal neuroprotection and preconditioning is mainly restricted to animal models and in-vitro work. Therefore, an extrapolation from these experimental data to the clinically daily routine is still impossible. Almost all animal models on neuroprotective strategies, like pharmacological interventions, hypothermia and preconditioning against post-asphyctic brain injury, focus on the immediate, histological outcome [124-126]. Neuroprotection is typically established by quantifying the reduction in infarct volume or the degree of cell death a few days after the insult, i.e. 1 week or shorter [124-126]. These methods are often unable to reliably detect more subtle morphological changes. In addition, post-asphyctic injury is a dynamic process extending up to weeks or even months after the initial insult $[58,152,154]$, which does not necessarily result in extensive or explicit brain damage on a morphological level $[89,155]$. These might be reasons why some clinical neuroprotective strate- 
gies failed in the long run, despite good protective results in short-term, morphological animal studies [158]. Some of the protective strategies might just delay the injury rather than providing a lasting protection and histological preservation does not necessarily mean that neuronal functioning is preserved $[156,157]$. Consequently, it is important to study both long-term functional and morphological consequences of neuroprotective strategies, like fetal asphyctic preconditioning, more extensively. A first effort was made in chapters 5-7 of this thesis. At the moment, the rather limited knowledge of the processes associated with neuroprotection renders it difficult to already suggest a specific therapeutic application.

\section{Current questions, open issues and future directions}

Despite increasing attention over the last 10 years, the fields of developmental asphyxia and neonatal preconditioning are still reasonably new and immature. A lot of issues remain tentative and hundreds of questions continue to be unanswered.

First, we must continue to identify the exact patterns, consequences and mechanisms of neonatal asphyxia and associated encephalopathy. Especially data on long-term processes and outcome are missing. The first three chapters in this thesis were a first step towards bridging this gap. A more extensive knowledge on these matters will enable scientists to acquire better insights that in the end might contribute to the development of therapeutic or preventive strategies. Furthermore, this information might help clinicians in the future to more accurately predict the long-term neurodevelopmental outcome of an individual child.

Second, we need additional data on the processes underlying preconditioning in the immature brain, as well as on its consequences. Evidently, a few key points need to be resolved before extrapolating these neuroprotective responses to the human patient. With respect to our studies in chapter 5-7, the optimal timing and the optimal duration of the fetal asphyctic stimulus still have to be determined. In addition, further studies are necessary to investigate how quickly neuroprotection is induced and how long exactly that neuroprotec- 
tion will persist. With this additional information, we might be able to optimize the stimulus in order to maximize the beneficial effects. Other questions are: Can a tolerant state also provide chronic neuroprotection? Are there potential side-effects yet to be identified? Is the preconditioning stimulus per se truly non-injurious? And is it really possible to selectively induce the beneficial effects of the noxious stimulus? Another issue to be solved is that clinicians rarely have the opportunity to intervene before the onset of asphyxia. To maximize the results, it might be that the potential agent has to be given prior to the insult, which at the moment cannot always be predicted yet. Therefore, postconditioning might be an interesting area of investigations as well. Moreover, we should keep in mind that the neuroprotective effects most likely involve several relevant biochemical signaling pathways that may consist of both dependent and independent actors that might produce wide and heterogeneous array of responses. For that reason, we have to find out if there is a serial pathway or a combination of serial and parallel pathways that converge in one or a few common effector molecules. But, will targeting one or a few of those individual effector molecules provide as much neuroprotection as the preconditioning stimulus itself?

In conclusion, many questions remain yet unanswered. As always, new findings raise more questions than answers.

\section{Concluding remarks}

\section{Fetal asphyxia: Friend or foe?}

The present work stresses that every coin has two sides. While severe fetal asphyxia is a serious threat to development (foe), causing both short and longterm functional and morphological impairments in the rat brain, mild fetal asphyxia can induce a long-lasting neuroprotection (friend). The question, however, whether the knowledge about fetal asphyctic preconditioning can contribute to a cure of neonatal encephalopathy remains yet unsolved. 


\section{References}

1. Gidday, J.M., Cerebral preconditioning and ischaemic tolerance. Nat Rev Neurosci, 2006. 7(6): p. 437-48.

2. Gunn, A.J., Cerebral hypothermia for prevention of brain injury following perinatal asphyxia. Curr Opin Pediatr, 2000. 12(2): p. 111-5.

3. Lawn, J.E., S. Cousens, and J. Zupan, 4 million neonatal deaths: when? Where? Why? Lancet, 2005. 365(9462): p. 891-900.

4. Johnston, M.V., et al., Neurobiology of hypoxic-ischemic injury in the developing brain. Pediatr Res, 2001. 49(6): p. 735-41.

5. Dirnagl, U., R.P. Simon, and J.M. Hallenbeck, Ischemic tolerance and endogenous neuroprotection. Trends Neurosci, 2003. 26(5): p. 248-54.

6. Low, J.A., Determining the contribution of asphyxia to brain damage in the neonate. J Obstet Gynaecol Res, 2004. 30(4): p. 276-86.

7. Pierrat, V., et al., Prevalence, causes, and outcome at 2 years of age of newborn encephalopathy: population based study. Arch Dis Child Fetal Neonatal Ed, 2005. 90(3): p. F257-61.

8. Low, J.A., Relationship of fetal asphyxia to neuropathology and deficits in children. Clin Invest Med, 1993. 16(2): p. 133-40.

9. Marchoudi, Z., Asphyxia in newborns requires better access to cool caps to improve outcomes, lower cost of treatment. 2008, Medical News Today.

10. Little, W.J., On the influence of abnormal parturition, difficult labours, premature birth and asphyxia neonatorum on the mental and physical condition of the child, especially in relation to deformities. Trans. Obstet. Soc. London, 1861. 3: p. 293-344.

11. Osler, W., The cerebral palsies of childhood. 1889, London: HK. Lewis.

12. Brann, A.W., Jr. and R.E. Myers, Central nervous system findings in the newborn monkey following severe in utero partial asphyxia. Neurology, 1975. 25(4): p. 327-38.

13. Myers, R.E., Fetal asphyxia due to umbilical cord compression. Metabolic and brain pathologic consequences. Biol Neonate, 1975. 26(1-2): p. 21-43.

14. Myers, R.E., Four patterns of perinatal brain damage and their conditions of occurrence in primates. Adv Neurol, 1975. 10: p. 223-34.

15. Eastman, N.J., Foetal blood studies III: The chemical nature of asphyxia neonatorum and its bearing on certain practical problems. Bull. J. Hop. Hosp., 
1932. 50: p. 1-39.

16. Sunshine, P., Perinatal asphyxia: an overview, in Fetal and neonatal brain injury, D.K. Stevenson, W.E. Benitz, and P. Sunshine, Editors. 2003, Cambridge University Press: Cambridge. p. 3-29.

17. Westgate, J.A., A.J. Gunn, and T.R. Gunn, Antecedents of neonatal encephalopathy with fetal acidaemia at term. Br J Obstet Gynaecol, 1999. 106(8): p. 774-82.

18. Parer, J.T., Effects of fetal asphyxia on brain cell structure and function: limits of tolerance. Comp Biochem Physiol A Mol Integr Physiol, 1998. $119(3)$ : p. 711-6.

19. Bennet, L., et al., The cardiovascular and cerebrovascular responses of the immature fetal sheep to acute umbilical cord occlusion. J Physiol, 1999. 517 ( Pt 1): p. 247-57.

20. Jensen, A. and R. Berger, Fetal circulatory responses to oxygen lack. J Dev Physiol, 1991. 16(4): p. 181-207.

21. Hanson, M.A., Do we now understand the control of the fetal circulation? Eur J Obstet Gynecol Reprod Biol, 1997. 75(1): p. 55-61.

22. Gunn, A.J., et al., The premature fetus: not as defenseless as we thought, but still paradoxically vulnerable? Dev Neurosci, 2001 . 23(3): p. 175-9.

23. van Handel, M., et al., Long-term cognitive and behavioral consequences of neonatal encephalopathy following perinatal asphyxia: a review. Eur J Pediatr, 2007. 166(7): p. 645-54.

24. Barnett, A., et al., Neurological and perceptual-motor outcome at 5 - 6 years of age in children with neonatal encephalopathy: relationship with neonatal brain MRI. Neuropediatrics, 2002. 33(5): p. 242-8.

25. Thornberg, E., et al., Birth asphyxia: incidence, clinical course and outcome in a Swedish population. Acta Paediatr, 1995. 84(8): p. 927-32.

26. Casteels, I., et al., Cortical visual impairment following perinatal hypoxia: clinicoradiologic correlation using magnetic resonance imaging. J Pediatr Ophthalmol Strabismus, 1997. 34(5): p. 297-305.

27. Jiang, Z.D., Long-term effect of perinatal and postnatal asphyxia on developing human auditory brainstem responses: peripheral hearing loss. Int J Pediatr Otorhinolaryngol, 1995. 33(3): p. 225-38.

28. Jiang, Z.D., et al., One-third of term babies after perinatal hypoxia-ischaemia have transient hearing impairment: dynamic change in hearing threshold during the neonatal period. Acta Paediatr, 2004. 93(1): p. 82-7. 
29. Jiang, Z.D., et al., Differential changes in peripheral and central components of the brain stem auditory evoked potentials during the neonatal period in term infants after perinatal hypoxia-ischemia. Ann Otol Rhinol Laryngol, 2004. 113(7): p. 571-6.

30. Brodsky, M.C., K.J. Fray, and C.M. Glasier, Perinatal cortical and subcortical visual loss: mechanisms of injury and associated ophthalmologic signs. Ophthalmology, 2002. 109(1): p. 85-94.

31. Martin, E. and A.J. Barkovich, Magnetic resonance imaging in perinatal asphyxia. Arch Dis Child Fetal Neonatal Ed, 1995. 72(1): p. F62-70.

32. Mercuri, E., et al., MRI lesions and infants with neonatal encephalopathy. Is the Apgar score predictive? Neuropediatrics, 2002. 33(3): p. 150-6.

33. Cowan, F., et al., Origin and timing of brain lesions in term infants with neonatal encephalopathy. Lancet, 2003. 361(9359): p. 736-42.

34. Mercuri, E., et al., Neonatal neurological examination in infants with hypoxic ischaemic encephalopathy: correlation with MRI findings. Neuropediatrics, 1999. 30(2): p. 83-9.

35. Yokochi, K. and S. Fujimoto, Magnetic resonance imaging in children with neonatal asphyxia: correlation with developmental sequelae. Acta Paediatr, 1996. 85(1): p. 88-95.

36. Squier, W. and F.M. Cowan, The value of autopsy in determining the cause of failure to respond to resuscitation at birth. Semin Neonatol, 2004. 9(4): p. 331-45.

37. Hunt, R.W., et al., Apparent diffusion coefficient in the posterior limb of the internal capsule predicts outcome after perinatal asphyxia. Pediatrics, 2004. 114(4): p. 999-1003.

38. Miller, S.P., et al., Patterns of brain injury in term neonatal encephalopathy. J Pediatr, 2005. 146(4): p. 453-60.

39. Pasternak, J.F. and M.T. Gorey, The syndrome of acute near-total intrauterine asphyxia in the term infant. Pediatr Neurol, 1998. 18(5): p. 391-8.

40. Sie, L.T., et al., MR patterns of hypoxic-ischemic brain damage after prenatal, perinatal or postnatal asphyxia. Neuropediatrics, 2000. 31(3): p. 128-36.

41. Alexander, G.E. and M.D. Crutcher, Functional architecture of basal ganglia circuits: neural substrates of parallel processing. Trends Neurosci, 1990. 13(7): p. 266-71.

42. de Vries, L.S. and F. Groenendaal, Neuroimaging in the preterm infant. Ment Retard Dev Disabil Res Rev, 2002. 8(4): p. 273-80. 
43. Back, S.A., et al., Late oligodendrocyte progenitors coincide with the developmental window of vulnerability for human perinatal white matter injury. J Neurosci, 2001. 21(4): p. 1302-12.

44. Haynes, R.L., et al., Nitrosative and oxidative injury to premyelinating oligodendrocytes in periventricular leukomalacia. J Neuropathol Exp Neurol, 2003. 62(5): p. 441-50.

45. McQuillen, P.S. and D.M. Ferriero, Selective vulnerability in the developing central nervous system. Pediatr Neurol, 2004. 30(4): p. 227-35.

46. Pierson, C.R., et al., Gray matter injury associated with periventricular leukomalacia in the premature infant. Acta Neuropathol, 2007. 114(6): p. 619-31.

47. Inder, T.E., et al., Abnormal cerebral structure is present at term in premature infants. Pediatrics, 2005. 115(2): p. 286-94.

48. Inder, T.E., et al., Defining the nature of the cerebral abnormalities in the premature infant: a qualitative magnetic resonance imaging study. J Pediatr, 2003. 143(2): p. 171-9.

49. Peterson, B.S., et al., Regional brain volume abnormalities and long-term cognitive outcome in preterm infants. Jama, 2000. 284(15): p. 1939-47.

50. Isaacs, E.B., et al., Hippocampal volume and everyday memory in children of very low birth weight. Pediatr Res, 2000. 47(6): p. 713-20.

51. Yager, J.Y., Animal models of hypoxic-ischemic brain damage in the newborn. Semin Pediatr Neurol, 2004. 11(1): p. 31-46.

52. Northington, F.J., Brief update on animal models of hypoxic-ischemic encephalopathy and neonatal stroke. Ilar J, 2006. 47(1): p. 32-8.

53. Ashwal, S. and W.J. Pearce, Animal models of neonatal stroke. Curr Opin Pediatr, 2001. 13(6): p. 506-16.

54. van Dijk, A.J., et al., Umbilical cord clamping in term piglets: a useful model to study perinatal asphyxia? Theriogenology, 2008. 70(4): p. 662-74.

55. Vannucci, R.C., J. Towfighi, and S.J. Vannucci, Hypoxic preconditioning and hypoxic-ischemic brain damage in the immature rat: pathologic and metabolic correlates. J Neurochem, 1998. 71(3): p. 1215-20.

56. Vannucci, R.C. and S.J. Vannucci, A model of perinatal hypoxic-ischemic brain damage. Ann N Y Acad Sci, 1997. 835: p. 234-49.

57. Van de Berg, W.D., et al., Perinatal asphyxia results in changes in presynaptic bouton number in striatum and cerebral cortex-a stereological and behavioral analysis. J Chem Neuroanat, 2000. 20(1): p. 71-82.

58. Van de Berg, W.D., et al., Perinatal asphyxia induced neuronal loss by apop- 
tosis in the neonatal rat striatum: a combined TUNEL and stereological study. Exp Neurol, 2002. 174(1): p. 29-36.

59. Dell'Anna, E., et al., Delayed neuronal death following perinatal asphyxia in rat. Exp Brain Res, 1997. 115(1): p. 105-15.

60. Northington, F.J., et al., Delayed neurodegeneration in neonatal rat thalamus after hypoxia-ischemia is apoptosis. J Neurosci, 2001. 21(6): p. 1931-8.

61. Northington, F.J., et al., Early Neurodegeneration after Hypoxia-Ischemia in Neonatal Rat Is Necrosis while Delayed Neuronal Death Is Apoptosis. Neurobiol Dis, 2001. 8(2): p. 207-19.

62. Cai, Z., J.B. Hutchins, and P.G. Rhodes, Intrauterine hypoxia-ischemia alters nitric oxide synthase expression and activity in fetal and neonatal rat brains. Brain Res Dev Brain Res, 1998. 109(2): p. 265-9.

63. Cai, Z., et al., Intrauterine hypoxia-ischemia increases N-methyl-Daspartate-induced cGMP formation and glutamate accumulation in cultured rat cerebellar granule cells. Pediatr Res, 1995. 38(1): p. 107-12.

64. Jensen, A., et al., Perinatal brain damage-from pathophysiology to prevention. Eur J Obstet Gynecol Reprod Biol, 2003. 110 Suppl 1: p. S70-9.

65. Berger, R. and Y. Garnier, Pathophysiology of perinatal brain damage. Brain Res Brain Res Rev, 1999. 30(2): p. 107-34.

66. Mishra, O.P. and M. Delivoria-Papadopoulos, Cellular mechanisms of hypoxic injury in the developing brain. Brain Res Bull, 1999. 48(3): p. 233-8.

67. Vexler, Z.S. and D.M. Ferriero, Molecular and biochemical mechanisms of perinatal brain injury. Semin Neonatol, 2001. 6(2): p. 99-108.

68. Vannucci, S.J. and H. Hagberg, Hypoxia-ischemia in the immature brain. J Exp Biol, 2004. 207(Pt 18): p. 3149-54.

69. Arundine, M. and M. Tymianski, Molecular mechanisms of calcium-dependent neurodegeneration in excitotoxicity. Cell Calcium, 2003. 34(4-5): p. 32537.

70. Berger, R., et al., Effects of acute asphyxia on brain energy metabolism in fetal guinea pigs near term. J Dev Physiol, 1991. 16(1): p. 9-11.

71. Berger, R., et al., Cerebral energy metabolism in immature and mature guinea pig fetuses during acute asphyxia. J Dev Physiol, 1992. 18(3): p. 125-8. 72. Mishra, O.P. and M. Delivoria-Papadopoulos, $\mathrm{Na}+\mathrm{K}+-\mathrm{ATPase}$ in developing fetal guinea pig brain and the effect of maternal hypoxia. Neurochem Res, 1988. 13(8): p. 765-70.

73. Benveniste, H., Glutamate, microdialysis, and cerebral ischemia: lost in trans- 
lation? Anesthesiology, 2009. 110(2): p. 422-5.

74. Benveniste, $\mathrm{H}_{\text {., }}$ et al., Elevation of the extracellular concentrations of glutamate and aspartate in rat hippocampus during transient cerebral ischemia monitored by intracerebral microdialysis. J Neurochem, 1984. 43(5): p. 136974.

75. Dirnagl, U., C. Iadecola, and M.A. Moskowitz, Pathobiology of ischaemic stroke: an integrated view. Trends Neurosci, 1999. 22(9): p. 391-7.

76. Delivoria-Papadopoulos, M. and O.P. Mishra, Mechanisms of perinatal cerebral injury in fetus and newborn. Ann N Y Acad Sci, 2000. 900: p. 159-68.

77. Pourcyrous, M., et al., Brain superoxide anion generation in asphyxiated piglets and the effect of indomethacin at therapeutic dose. Pediatr Res, 1993. 34(3): p. 366-9.

78. Kumar, A., et al., Free radical injury and blood-brain barrier permeability in hypoxic-ischemic encephalopathy. Pediatrics, 2008. 122(3): p. e722-7.

79. Kumar, A., et al., Oxidative stress in perinatal asphyxia. Pediatr Neurol, 2008. 38(3): p. 181-5.

80. Mishra, O.P. and M. Delivoria-Papadopoulos, Lipid peroxidation in developing fetal guinea pig brain during normoxia and hypoxia. Brain Res Dev Brain Res, 1989. 45(1): p. 129-35.

81. Mishra, O.P. and M. Delivoria-Papadopoulos, Anti-oxidant enzymes in fetal guinea pig brain during development and the effect of maternal hypoxia. Brain Res, 1988. 470(2): p. 173-9.

82. Takashima, S., et al., Immunohistochemistry of superoxide dismutase-1 in developing human brain. Brain Dev, 1990. 12(2): p. 211-3.

83. Inder, T.E., et al., Lipid peroxidation as a measure of oxygen free radical damage in the very low birthweight infant. Arch Dis Child Fetal Neonatal Ed, 1994. 70(2): p. F107-11.

84. Smith, C.V., et al., Oxidant stress responses in premature infants during exposure to hyperoxia. Pediatr Res, 1993. 34(3): p. 360-5.

85. Evans, P.J., et al., Bleomycin-detectable iron in the plasma of premature and full-term neonates. FEBS Lett, 1992. 303(2-3): p. 210-2.

86. Palmer, C. and R.C. Vannucci, Cellular and molecular biology of perinatal hypoxic-ischemic encephalopathy, in Fetal and neonatal brain injury, D.K. Stevenson, W.E. Benitz, and P. Sunshine, Editors. 2003, Press syndicate of the university of Cambridge: Cambridge. p. 58-82.

87. Martin, L.J., et al., Neurodegeneration in excitotoxicity, global cerebral is- 
chemia, and target deprivation: A perspective on the contributions of apoptosis and necrosis. Brain Res Bull, 1998. 46(4): p. 281-309.

88. Speiser, Z., et al., Hyperactivity in rats following postnatal anoxia. Behav Brain Res, 1983. 7(3): p. 379-82.

89. Balduini, W., et al., Long-lasting behavioral alterations following a hypoxic/ischemic brain injury in neonatal rats. Brain Res, 2000. 859(2): p. 318-25. 90. Hershkowitz, M., V.E. Grimm, and Z. Speiser, The effects of postnatal anoxia on behaviour and on the muscarinic and beta-adrenergic receptors in the hippocampus of the developing rat. Brain Res, 1983. 283(2-3): p. 147-55.

91. Dell'Anna, M.E., et al., Neonatal anoxia induces transitory hyperactivity, permanent spatial memory deficits and CA1 cell density reduction in developing rats. Behav Brain Res, 1991. 45(2): p. 125-34.

92. Iuvone, L., M.C. Geloso, and E. Dell'Anna, Changes in open field behavior, spatial memory, and hippocampal parvalbumin immunoreactivity following enrichment in rats exposed to neonatal anoxia. Exp Neurol, 1996. 139(1): p. 2533.

93. Cai, Z., et al., Prenatal hypoxia-ischemia alters expression and activity of nitric oxide synthase in the young rat brain and causes learning deficits. Brain Res Bull, 1999. 49(5): p. 359-65.

94. Jansen, E.M. and W.C. Low, Long-term effects of neonatal ischemic-hypoxic brain injury on sensorimotor and locomotor tasks in rats. Behav Brain Res, 1996. 78(2): p. 189-94.

95. Jansen, E.M. and W.C. Low, Quantitative analysis of contralateral hemisphere hypertrophy and sensorimotor performance in adult rats following unilateral neonatal ischemic-hypoxic brain injury. Brain Res, 1996. 708(1-2): p. 93-9.

96. Bona, E., B.B. Johansson, and H. Hagberg, Sensorimotor function and neuropathology five to six weeks after hypoxia-ischemia in seven-day-old rats. Pediatr Res, 1997. 42(5): p. 678-83.

97. Simola, N., et al., Acute perinatal asphyxia impairs non-spatial memory and alters motor coordination in adult male rats. Exp Brain Res, 2008. 185(4): p. 595-601.

98. Ikeda, T., et al., Selective and long-term learning impairment following neonatal hypoxic-ischemic brain insult in rats. Behav Brain Res, 2001. 118(1): p. 17-25.

99. Wagner, B.P., J. Nedelcu, and E. Martin, Delayed postischemic hypother- 
mia improves long-term behavioral outcome after cerebral hypoxia-ischemia in neonatal rats. Pediatr Res, 2002. 51(3): p. 354-60.

100. Young, R.S., et al., Behavioral performance of rats following neonatal hypoxia-ischemia. Stroke, 1986. 17(6): p. 1313-6.

101. Boksa, P., A. Krishnamurthy, and W. Brooks, Effects of a period of asphyxia during birth on spatial learning in the rat. Pediatr Res, 1995. 37(4 Pt 1): p. 48996.

102. Weitzdoerfer, R., et al., Long-term sequelae of perinatal asphyxia in the aging rat. Cell Mol Life Sci, 2002. 59(3): p. 519-26.

103. Weitzdoerfer, R., et al., Long-term influence of perinatal asphyxia on the social behavior in aging rats. Gerontology, 2004. 50(4): p. 200-5.

104. Volpe, J.J., Perinatal brain injury: from pathogenesis to neuroprotection. Ment Retard Dev Disabil Res Rev, 2001. 7(1): p. 56-64.

105. Gluckman, P.D., C.S. Pinal, and A.J. Gunn, Hypoxic-ischemic brain injury in the newborn: pathophysiology and potential strategies for intervention. Semin Neonatol, 2001. 6(2): p. 109-20.

106. van Bel, F. and F. Groenendaal, Long-term pharmacologic neuroprotection after birth asphyxia: where do we stand? Neonatology, 2008. 94(3): p. 203-10.

107. Gunn, A.J. and P.D. Gluckman, Head cooling for neonatal encephalopathy: the state of the art. Clin Obstet Gynecol, 2007. 50(3): p. 636-51.

108. Gunn, A.J., et al., Neuroprotection with prolonged head cooling started before postischemic seizures in fetal sheep. Pediatrics, 1998. 102(5): p. 1098106.

109. Gluckman, P.D., et al., Selective head cooling with mild systemic hypothermia after neonatal encephalopathy: multicentre randomised trial. Lancet, 2005. 365(9460): p. 663-70.

110. Shankaran, S., Neonatal encephalopathy: treatment with hypothermia. J Neurotrauma, 2009. 26(3): p. 437-43.

111. Shankaran, S., et al., Whole-body hypothermia for neonatal encephalopathy: animal observations as a basis for a randomized, controlled pilot study in term infants. Pediatrics, 2002. $110(2$ Pt 1): p. 377-85.

112. Shankaran, S. and A.R. Laptook, Hypothermia as a treatment for birth asphyxia. Clin Obstet Gynecol, 2007. 50(3): p. 624-35.

113. Shankaran, S., et al., Whole-body hypothermia for neonates with hypoxicischemic encephalopathy. N Engl J Med, 2005. 353(15): p. 1574-84. 
114. Shankaran, S., et al., Outcomes of safety and effectiveness in a multicenter randomized, controlled trial of whole-body hypothermia for neonatal hypoxic-ischemic encephalopathy. Pediatrics, 2008. 122(4): p. e791-8.

115. Murry, C.E., R.B. Jennings, and K.A. Reimer, Preconditioning with ischemia: a delay of lethal cell injury in ischemic myocardium. Circulation, 1986. 74(5): p. 1124-36.

116. Perez-Pinzon, M.A., et al., Rapid preconditioning protects rats against ischemic neuronal damage after 3 but not 7 days of reperfusion following global cerebral ischemia. J Cereb Blood Flow Metab, 1997. 17(2): p. 175-82.

117. Perez-Pinzon, M.A., et al., Rapid ischemic preconditioning protects rats from cerebral anoxia/ischemia. Adv Exp Med Biol, 1997. 428: p. 155-61.

118. Kitagawa, K., et al., 'Ischemic tolerance' phenomenon found in the brain. Brain Res, 1990. 528(1): p. 21-4.

119. Jones, N.M. and M. Bergeron, Hypoxic preconditioning induces changes in HIF-1 target genes in neonatal rat brain. J Cereb Blood Flow Metab, 2001. 21(9): p. $1105-14$.

120. Mallard, C. and H. Hagberg, Inflammation-induced preconditioning in the immature brain. Semin Fetal Neonatal Med, 2007. 12(4): p. 280-6.

121. Xiao, F., et al., Reduced nitric oxide is involved in prenatal ischemia-induced tolerance to neonatal hypoxic-ischemic brain injury in rats. Neurosci Lett, 2000. 285(1): p. 5-8.

122. Wada, T., T. Kondoh, and N. Tamaki, Ischemic "cross" tolerance in hypoxic ischemia of immature rat brain. Brain Res, 1999. 847(2): p. 299-307.

123. Zhao, P., et al., Isoflurane preconditioning improves long-term neurologic outcome after hypoxic-ischemic brain injury in neonatal rats. Anesthesiology, 2007. 107(6): p. 963-70.

124. Gidday, J.M., et al., Neuroprotection from ischemic brain injury by hypoxic preconditioning in the neonatal rat. Neurosci Lett, 1994. 168(1-2): p. 221-4. 125. Ota, A., et al., Hypoxic-ischemic tolerance phenomenon observed in neonatal rat brain. Am J Obstet Gynecol, 1998. 179(4): p. 1075-8.

126. Cantagrel, S., et al., Hypoxic preconditioning reduces apoptosis in a rat model of immature brain hypoxia-ischaemia. Neurosci Lett, 2003. 347(2): p. 106-10.

127. Jones, N.M., et al., Long-term functional and protective actions of preconditioning with hypoxia, cobalt chloride, and desferrioxamine against hypoxic-ischemic injury in neonatal rats. Pediatr Res, 2008. 63(6): p. 620-4. 
128. Gustavsson, M., et al., Hypoxic preconditioning confers long-term reduction of brain injury and improvement of neurological ability in immature rats. Pediatr Res, 2005. 57(2): p. 305-9.

129. Lin, W.Y., et al., CREB activation in the rapid, intermediate, and delayed ischemic preconditioning against hypoxic-ischemia in neonatal rat. J Neurochem, 2009. 108(4): p. 847-59.

130. Cai, Z., J.D. Fratkin, and P.G. Rhodes, Prenatal ischemia reduces neuronal injury caused by neonatal hypoxia-ischemia in rats. Neuroreport, 1997. 8(6): p. 1393-8.

131. Wang, X., et al., Potential role of IGF-I in hypoxia tolerance using a rat hypoxic-ischemic model: activation of hypoxia-inducible factor 1 alpha. Pediatr Res, 2004. 55(3): p. 385-94.

132. Bernaudin, M., et al., Brain genomic response following hypoxia and reoxygenation in the neonatal rat. Identification of genes that might contribute to hypoxia-induced ischemic tolerance. J Biol Chem, 2002. 277(42): p. 3972838.

133. Jones, N.M., et al., Hypoxic preconditioning produces differential expression of hypoxia-inducible factor- 1 alpha (HIF-1 alpha) and its regulatory enzyme HIF prolyl hydroxylase 2 in neonatal rat brain. Neurosci Lett, 2006. 404(1-2): p. 72-7.

134. Laudenbach, V., et al., Neonatal hypoxic preconditioning involves vascular endothelial growth factor. Neurobiol Dis, 2007. 26(1): p. 243-52.

135. Brucklacher, R.M., R.C. Vannucci, and S.J. Vannucci, Hypoxic preconditioning increases brain glycogen and delays energy depletion from hypoxiaischemia in the immature rat. Dev Neurosci, 2002. 24(5): p. 411-7.

136. Lee, H.T., et al., cAMP response element-binding protein activation in ligation preconditioning in neonatal brain. Ann Neurol, 2004. 56(5): p. 611-23.

137. Ikeda, T., et al., Important role of 72-kd heat shock protein expression in the endothelial cell in acquisition of hypoxic-ischemic tolerance in the immature rat. Am J Obstet Gynecol, 2000. 182(2): p. 380-6.

138. Chang, Y.C. and C.C. Huang, Perinatal brain injury and regulation of transcription. Curr Opin Neurol, 2006. 19(2): p. 141-7.

139. Williamson, C.L., et al., Mitochondria protection from hypoxia/reoxygenation injury with mitochondria heat shock protein 70 overexpression. Am J Physiol Heart Circ Physiol, 2008. 294(1): p. H249-56.

140. Zhao, P. and Z. Zuo, Prenatal hypoxia-induced adaptation and neuro- 
protection that is inducible nitric oxide synthase-dependent. Neurobiol Dis, 2005. 20(3): p. 871-80.

141. Vannucci, R.C. and T.E. Duffy, Carbohydrate metabolism in fetal and neonatal rat brain during anoxia and recovery. Am J Physiol, 1976. 230(5): p. 1269-75.

142. Dawes, G.S., et al., Some observations on foetal and new-born rhesus monkeys. J Physiol, 1960. 152: p. 271-98.

143. Dawes, G.S., J.C. Mott, and H.J. Shelley, The importance of cardiac glycogen for the maintenance of life in foetal lambs and newborn animals during anoxia. J Physiol, 1959. 146(3): p. 516-38.

144. Lynch, J.K. and K.B. Nelson, Epidemiology of perinatal stroke. Curr Opin Pediatr, 2001. 13(6): p. 499-505.

145. Clancy, B., et al., Extrapolating brain development from experimental species to humans. Neurotoxicology, 2007. 28(5): p. 931-7.

146. Clancy, B., R.B. Darlington, and B.L. Finlay, Translating developmental time across mammalian species. Neuroscience, 2001. 105(1): p. 7-17.

147. Dobbing, J. and J. Sands, Comparative aspects of the brain growth spurt. Early Hum Dev, 1979. 3(1): p. 79-83.

148. Romijn, H.J., M.A. Hofman, and A. Gramsbergen, At what age is the developing cerebral cortex of the rat comparable to that of the full-term newborn human baby? Early Hum Dev, 1991. 26(1): p. 61-7.

149. Skrablin, S., et al., Perinatal factors associated with the neurologic impairment of children born preterm. Int J Gynaecol Obstet, 2008. 102(1): p. 12-8.

150. Evrard, P., P. Gressens, and J.J. Volpe, New concepts to understand the neurological consequences of subcortical lesions in the premature brain. Biol Neonate, 1992. 61(1): p. 1-3.

151. Volpe, J.J., Brain injury in the premature infant-current concepts of pathogenesis and prevention. Biol Neonate, 1992. 62(4): p. 231-42.

152. Strackx, E., et al., Fetal asphyxia leads to a decrease in dorsal raphe serotonergic neurons. Dev Neurosci, 2008. 30(5): p. 358-66.

153. Strackx, E., et al., A combined behavioral and morphological study on the effects of fetal asphyxia on the nigrostriatal dopaminergic system in adult rats. Exp Neurol, 2008. 211 (2): p. 413-22.

154. Geddes, R., R.C. Vannucci, and S.J. Vannucci, Delayed cerebral atrophy following moderate hypoxia-ischemia in the immature rat. Dev Neurosci, 2001. 23(3): p. 180-5. 
155. Hori, N. and D.O. Carpenter, Functional and morphological changes induced by transient in vivo ischemia. Exp Neurol, 1994. 129(2): p. 279-89.

156. Trescher, W.H., S. Ishiwa, and M.V. Johnston, Brief post-hypoxicischemic hypothermia markedly delays neonatal brain injury. Brain Dev, 1997. 19(5): p. 326-38.

157. Kawaguchi, M., et al., Isoflurane delays but does not prevent cerebral infarction in rats subjected to focal ischemia. Anesthesiology, 2000. 92(5): p. $1335-42$.

158. Corbett, D. and S. Nurse, The problem of assessing effective neuroprotection in experimental cerebral ischemia. Prog Neurobiol, 1998. 54(5): p. 531 48.

159. Hill, A., Current concepts of hypoxic-ischemic cerebral injury in the term newborn. Pediatr Neurol, 1991. 7(5): p. 317-25.

160. Younkin, D.P., Hypoxic-ischemic brain injury of the newborn-statement of the problem and overview. Brain Pathol, 1992. 2(3): p. 209-10.

161. Low, J.A., Reflections on the occurrence and significance of antepartum fetal asphyxia. Best Pract Res Clin Obstet Gynaecol, 2004. 18(3): p. 375-82.

162. Hultman, C.M., P. Sparen, and S. Cnattingius, Perinatal risk factors for infantile autism. Epidemiology, 2002. 13(4): p. 417-23.

163. Lewis, S.W. and R.M. Murray, Obstetric complications, neurodevelopmental deviance, and risk of schizophrenia. J Psychiatr Res, 1987. 21(4): p. 41321.

164. Cannon, M., P.B. Jones, and R.M. Murray, Obstetric complications and schizophrenia: historical and meta-analytic review. Am J Psychiatry, 2002. 159(7): p. 1080-92.

165. Faraone, S.V. and J. Biederman, Neurobiology of attention-deficit hyperactivity disorder. Biol Psychiatry, 1998. 44(10): p. 951-8.

166. Loidl, C.F., et al., Effects of hypothermia and gender on survival and behavior after perinatal asphyxia in rats. Physiol Behav, 2000. 68(3): p. 263-9.

167. Nyakas, C., B. Buwalda, and P.G. Luiten, Hypoxia and brain development. Prog Neurobiol, 1996. 49(1): p. 1-51.

168. Vannucci, R.C. and S.J. Vannucci, Perinatal hypoxic-ischemic brain damage: evolution of an animal model. Dev Neurosci, 2005. 27(2-4): p. 81-6.

169. Casaer, P., Old and new facts about perinatal brain development. J Child Psychol Psychiatry, 1993. 34(1): p. 101-9.

170. Naeye, R.L. and E.C. Peters, Antenatal hypoxia and low IQ values. Am J Dis 
Child, 1987. 141(1): p. 50-4.

171. Lindahl, E., et al., Neonatal risk factors and later neurodevelopmental disturbances. Dev Med Child Neurol, 1988. 30(5): p. 571-89.

172. Uvebrant, P. and G. Hagberg, Intrauterine growth in children with cerebral palsy. Acta Paediatr, 1992. 81(5): p. 407-12.

173. Klawitter, V., et al., Plasticity of the central nervous system (CNS) following perinatal asphyxia: Does nicotinamide provide neuroprotection? Amino Acids, 2006. 31(4): p. 377-84.

174. Barkovich, A.J., MR and CT evaluation of profound neonatal and infantile asphyxia. AJNR Am J Neuroradiol, 1992. 13(3): p. 959-72; discussion 973-5.

175. Pasternak, J.F., T.A. Predey, and M.A. Mikhael, Neonatal asphyxia: vulnerability of basal ganglia, thalamus, and brainstem. Pediatr Neurol, 1991. 7(2): p. 147-9.

176. Serrano, M., et al., Perinatal asphyxia may cause reduction in CSF dopamine metabolite concentrations. Neurology, 2007. 69(3): p. 311-3.

177. Klawitter, V., et al., Plasticity of basal ganglia neurocircuitries following perinatal asphyxia: effect of nicotinamide. Exp Brain Res, 2007.

178. Chen, Y., et al., Perinatal asphyxia-induced changes in rat brain tyrosine hydroxylase-immunoreactive cell body number: effects of nicotine treatment. Neurosci Lett, 1997. $221(2-3)$ : p. 77-80.

179. Chen, Y., et al., Perinatal asphyxia induces long-term changes in dopamine D1, D2, and D3 receptor binding in the rat brain. Exp Neurol, 1997. 146(1): p. 74-80.

180. Loidl, C.F., et al., Long-term effects of perinatal asphyxia on basal ganglia neurotransmitter systems studied with microdialysis in rat. Neurosci Lett, 1994. 175(1-2): p. 9-12.

181. Oo, T.F., C. Henchcliffe, and R.E. Burke, Apoptosis in substantia nigra following developmental hypoxic-ischemic injury. Neuroscience, 1995. 69(3): p. 893-901.

182. Burke, R.E., et al., Neonatal hypoxic-ischemic or excitotoxic striatal injury results in a decreased adult number of substantia nigra neurons. Neuroscience, 1992. 50(3): p. 559-69.

183. Groenewegen, H.J., The basal ganglia and motor control. Neural Plast, 2003. 10(1-2): p. 107-20.

184. Marchand, R. and L.J. Poirier, Isthmic origin of neurons of the rat substantia nigra. Neuroscience, 1983. 9(2): p. 373-81. 
185. Lauder, J.M. and F.E. Bloom, Ontogeny of monoamine neurons in the locus coeruleus, Raphe nuclei and substantia nigra of the rat. I. Cell differentiation. J Comp Neurol, 1974. 155(4): p. 469-81.

186. Lauder, J.M. and F.E. Bloom, Ontogeny of monoamine neurons in the locus coeruleus, raphe nuclei and substantia nigra of the rat. II. Synaptogenesis. J Comp Neurol, 1975. 163(3): p. 251-64.

187. Gates, M.A., et al., Re-examining the ontogeny of substantia nigra dopamine neurons. Eur J Neurosci, 2006. 23(5): p. 1384-90.

188. Shults, C.W., et al., Dopaminergic cells align along radial glia in the developing mesencephalon of the rat. Neuroscience, 1990. 38(2): p. 427-36.

189. Specht, L.A., et al., Light-microscopic immunocytochemical localization of tyrosine hydroxylase in prenatal rat brain. II. Late ontogeny. J Comp Neurol, 1981. 199(2): p. 255-76.

190. Burke, R.E., Postnatal developmental programmed cell death in dopamine neurons. Ann N Y Acad Sci, 2003. 991: p. 69-79.

191. El-Khodor, B.F. and P. Boksa, Differential vulnerability of male versus female rats to long-term effects of birth insult on brain catecholamine levels. Exp Neurol, 2003. 182(1): p. 208-19.

192. Zhang, Y.Q., et al., Effects of gender and estradiol treatment on focal brain ischemia. Brain Res, 1998. 784(1-2): p. 321-4.

193. Schmitz, C. and P.R. Hof, Design-based stereology in neuroscience. Neuroscience, 2005. 130(4): p. 813-31.

194. Kuczenski, R., et al., Hippocampus norepinephrine, caudate dopamine and serotonin, and behavioral responses to the stereoisomers of amphetamine and methamphetamine. J Neurosci, 1995. 15(2): p. 1308-17.

195. Chen, Y., et al., Nicotine treatment counteracts perinatal asphyxia-induced changes in the mesostriatal/limbic dopamine systems and in motor behaviour in the four-week-old male rat. Neuroscience, 1995. 68(2): p. 531-8.

196. Vaillancourt, C. and P. Boksa, Birth insult alters dopamine-mediated behavior in a precocial species, the guinea pig. Implications for schizophrenia. Neuropsychopharmacology, 2000. 23(6): p. 654-66.

197. Fan, L.W., et al., Hypoxia-ischemia induced neurological dysfunction and brain injury in the neonatal rat. Behav Brain Res, 2005. 165(1): p. 80-90.

198. Young, C., et al., Potential of ketamine and midazolam, individually or in combination, to induce apoptotic neurodegeneration in the infant mouse brain. Br J Pharmacol, 2005. 146(2): p. 189-97. 
199. Rudin, M., et al., Single-dose ketamine administration induces apoptosis in neonatal mouse brain. J Basic Clin Physiol Pharmacol, 2005. 16(4): p. 231-43. 200. Ferro, M.M., et al., Neuroprotective effect of ketamine/xylazine on two rat models of Parkinson's disease. Braz J Med Biol Res, 2007. 40(1): p. 89-96.

201. Bjorklund, A. and S.B. Dunnett, Dopamine neuron systems in the brain: an update. Trends Neurosci, 2007. 30(5): p. 194-202.

202. Johnston, M.V., Hypoxic and ischemic disorders of infants and children. Lecture for 38th meeting of Japanese Society of Child Neurology, Tokyo, Japan, July 1996. Brain Dev, 1997. 19(4): p. 235-9.

203. Ungethum, U., et al., Effects of perinatal asphyxia on the mesostriatal/mesolimbic dopamine system of neonatal and 4-week-old male rats. Exp Brain Res, 1996. $112(3)$ : p. 403-10.

204. Chen, Y., et al., Short- and long-term effects of perinatal asphyxia on monoamine, amino acid and glycolysis product levels measured in the basal ganglia of the rat. Brain Res Dev Brain Res, 1997. 104(1-2): p. 19-30.

205. Morales, P., et al., Perinatal asphyxia impairs connectivity and dopamine neurite branching in organotypic triple culture from rat substantia nigra, neostriatum and neocortex. Neurosci Lett, 2003. 348(3): p. 175-9.

206. Klawitter, V., et al., Effects of perinatal asphyxia on cell survival, neuronal phenotype and neurite growth evaluated with organotypic triple cultures. Amino Acids, 2005. 28(2): p. 149-55.

207. Norton, W.T., et al., Quantitative aspects of reactive gliosis: a review. Neurochem Res, 1992. 17(9): p. 877-85.

208. Dusart, I., S. Marty, and M. Peschanski, Glial changes following an excitotoxic lesion in the CNS-II. Astrocytes. Neuroscience, 1991. 45(3): p. 541-9.

209. da Cunha, A., et al., Control of astrocytosis by interleukin-1 and transforming growth factor-beta 1 in human brain. Brain Res, 1993. 631(1): p. 3945.

210. Capani, F., et al., Changes in reactive oxygen species (ROS) production in rat brain during global perinatal asphyxia: an ESR study. Brain Res, 2001 . 914/12): p. 204-7.

211. Rostworowski, M., et al., Astrogliosis in the neonatal and adult murine brain post-trauma: elevation of inflammatory cytokines and the lack of requirement for endogenous interferon-gamma. J Neurosci, 1997. 17(10): p. 3664-74.

212. Reier, P., Gliosis following CNS injury: the anatomy of astrocytic scars and 
their influences on axonal elongation. Astrocytes, Cell Biology and Pathology of Astrocytes., ed. V.A. Fedoroff S. 1986, New York: Academic Press. 263-324. 213. Montgomery, D.L., Astrocytes: form, functions, and roles in disease. Vet Pathol, 1994. 31(2): p. 145-67.

214. Magistretti, P.J. and L. Pellerin, Cellular bases of brain energy metabolism and their relevance to functional brain imaging: evidence for a prominent role of astrocytes. Cereb Cortex, 1996. 6(1): p. 50-61.

215. Ment, L.R., et al., Association of chronic sublethal hypoxia with ventriculomegaly in the developing rat brain. Brain Res Dev Brain Res, 1998. 111 (2): p. 197-203.

216. Turner, C.P., et al., A1 adenosine receptors mediate hypoxia-induced ventriculomegaly. Proc Natl Acad Sci U S A, 2003. 100(20): p. 11718-22.

217. Rademakers, R.P., et al., Central cortico-subcortical involvement: a distinct pattern of brain damage caused by perinatal and postnatal asphyxia in term infants. J Comput Assist Tomogr, 1995. 19(2): p. 256-63.

218. Ment, L.R., et al., The etiology and outcome of cerebral ventriculomegaly at term in very low birth weight preterm infants. Pediatrics, 1999. 104(2 Pt 1): p. 243-8.

219. Barkovich, A.J. and S.K. Sargent, Profound asphyxia in the premature infant: imaging findings. AJNR Am J Neuroradiol, 1995. 16(9): p. 1837-46.

220. Back, S.A. and S.A. Rivkees, Emerging concepts in periventricular white matter injury. Semin Perinatol, 2004. 28(6): p. 405-14.

221. Stewart, A. and V. Kirkbride, Very preterm infants at fourteen years: relationship with neonatal ultrasound brain scans and neurodevelopmental status at one year. Acta Paediatr Suppl, 1996. 416: p. 44-7.

222. Ouahba, J., et al., Prenatal isolated mild ventriculomegaly: outcome in 167 cases. Bjog, 2006. 113(9): p. 1072-9.

223. Barlow, B.K., et al., The gestational environment and Parkinson's disease: evidence for neurodevelopmental origins of a neurodegenerative disorder. Reprod Toxicol, 2007. 23(3): p. 457-70.

224. Rees, S. and T. Inder, Fetal and neonatal origins of altered brain development. Early Hum Dev, 2005. 81(9): p. 753-61.

225. Salhab, W.A. and J.M. Perlman, Severe fetal acidemia and subsequent neonatal encephalopathy in the larger premature infant. Pediatr Neurol, 2005. 32(1): p. 25-9.

226. Hoeger, H., et al., Long term neurological and behavioral effects of graded 
perinatal asphyxia in the rat. Life Sci, 2000. 66(10): p. 947-62.

227. Strackx, E., et al., A combined behavioral and morphological study on the effects of fetal asphyxia on the nigrostriatal dopaminergic system in adult rats. Exp Neurol, 2008. 211 (2): p. 413-422.

228. Ressler, K.J. and C.B. Nemeroff, Role of serotonergic and noradrenergic systems in the pathophysiology of depression and anxiety disorders. Depress Anxiety, 2000. 12 Suppl 1: p. 2-19.

229. Stockmeier, C.A., Involvement of serotonin in depression: evidence from postmortem and imaging studies of serotonin receptors and the serotonin transporter. J Psychiatr Res, 2003. 37(5): p. 357-73.

230. Arango, V., M.D. Underwood, and J.J. Mann, Serotonin brain circuits involved in major depression and suicide. Prog Brain Res, 2002. 136: p. 443-53. 231. Graeff, F.G., et al., Role of 5-HT in stress, anxiety, and depression. Pharmacol Biochem Behav, 1996. 54(1): p. 129-41.

232. Whitaker-Azmitia, P.M., Serotonin and brain development: role in human developmental diseases. Brain Res Bull, 2001. 56(5): p. 479-85.

233. Di Pino, G.D., et al., Roles for Serotonin in Neurodevelopment: More than just Neural Transmission. Current Neuropharmacology, 2004. 2(4): p. 403-417. 234. Michelsen, K.A., C. Schmitz, and H.W. Steinbusch, The dorsal raphe nucleus-from silver stainings to a role in depression. Brain Res Rev, 2007. 55(2): p. 329-42.

235. Lieben, C.K., et al., Acute tryptophan depletion induced by a gelatin-based mixture impairs object memory but not affective behavior and spatial learning in the rat. Behav Brain Res, 2004. 151(1-2): p. 53-64.

236. Paxinos, G. and C. Watson, The rat brain in stereotaxic coordinates. 1986, San Diego: Academic Press.

237. De Haan, H.H., A.J. Gunn, and P.D. Gluckman, Experiments in perinatal brain injury: What have we learnt? Prenatal and Neonatal Medicine 1996. 1(1): p. 16-15.

238. Bennet, L., et al., Relationship between evolving epileptiform activity and delayed loss of mitochondrial activity after asphyxia measured by near-infrared spectroscopy in preterm fetal sheep. J Physiol, 2006. 572(Pt 1): p. 141-54.

239. Aitken, A.R. and I. Tork, Early development of serotonin-containing neurons and pathways as seen in wholemount preparations of the fetal rat brain. J Comp Neurol, 1988. 274(1): p. 32-47.

240. Lidov, H.G. and M.E. Molliver, An immunohistochemical study of serotonin 
neuron development in the rat: ascending pathways and terminal fields. Brain Res Bull, 1982. 8(4): p. 389-430.

241. Rubenstein, J.L., Development of serotonergic neurons and their projections. Biol Psychiatry, 1998. 44(3): p. 145-50.

242. Wallace, J.A. and J.M. Lauder, Development of the serotonergic system in the rat embryo: an immunocytochemical study. Brain Res Bull, 1983. 10(4): p. 459-79.

243. Flood, D.G. and P.D. Coleman, Neuron numbers and sizes in aging brain: comparisons of human, monkey, and rodent data. Neurobiol Aging, 1988. 9/56): p. 453-63.

244. Henderson, G., B.E. Tomlinson, and P.H. Gibson, Cell counts in human cerebral cortex in normal adults throughout life using an image analysing computer. J Neurol Sci, 1980. 46(1): p. 113-36.

245. Brizzee, K.R. and J.M. Ordy, Age pigments, cell loss and hippocampal function. Mech Ageing Dev, 1979. 9(1-2): p. 143-62.

246. Mani, R.B., J.B. Lohr, and D.V. Jeste, Hippocampal pyramidal cells and aging in the human: a quantitative study of neuronal loss in sectors CA 1 to CA4. Exp Neurol, 1986. 94(1): p. 29-40.

247. Sapolsky, R.M., L.C. Krey, and B.S. McEwen, Prolonged glucocorticoid exposure reduces hippocampal neuron number: implications for aging. J Neurosci, 1985. 5(5): p. 1222-7.

248. Esiri, M.M., Ageing and the brain. J Pathol, 2007. 211 (2): p. 181-7.

249. Morrison, J.H. and P.R. Hof, Selective vulnerability of corticocortical and hippocampal circuits in aging and Alzheimer's disease. Prog Brain Res, 2002. 136: p. 467-86.

250. Eysel, U.T. and U. Wolfhard, The effects of partial retinal lesions on activity and size of cells in the dorsal lateral geniculate nucleus. J Comp Neurol, 1984. 229(2): p. 301-9.

251. Kiernan, J.A. and A.J. Hudson, Changes in sizes of cortical and lower motor neurons in amyotrophic lateral sclerosis. Brain, 1991. 114 ( Pt 2): p. 84353.

252. Mountjoy, C.Q., et al., Cortical neuronal counts in normal elderly controls and demented patients. Neurobiol Aging, 1983. 4(1): p. 1-11.

253. Nyakas, C., et al., Postnatal development of hippocampal and neocortical cholinergic and serotonergic innervation in rat: effects of nitrite-induced prenatal hypoxia and nimodipine treatment. Neuroscience, 1994. 59(3): p. 541-59. 
254. van Luijtelaar, M.G., J.A. Tonnaer, and H.W. Steinbusch, Aging of the serotonergic system in the rat forebrain: an immunocytochemical and neurochemical study. Neurobiol Aging, 1992. 13(2): p. 201-15.

255. Graeff, F.G., M.B. Viana, and P.O. Mora, Dual role of 5-HT in defense and anxiety. Neurosci Biobehav Rev, 1997. 21(6): p. 791-9.

256. Boguszewski, P. and J. Zagrodzka, Emotional changes related to age in rats-a behavioral analysis. Behav Brain Res, 2002. 133(2): p. 323-32.

257. Indredavik, M.S., et al., Psychiatric symptoms and disorders in adolescents with low birth weight. Arch Dis Child Fetal Neonatal Ed, 2004. 89(5): p. F44550.

258. Meltzer, C.C., et al., Serotonin in aging, late-life depression, and Alzheimer's disease: the emerging role of functional imaging. Neuropsychopharmacology, 1998. 18(6): p. 407-30.

259. Marlow, N., et al., Neuropsychological and educational problems at school age associated with neonatal encephalopathy. Arch Dis Child Fetal Neonatal Ed, 2005. 90(5): p. F380-7.

260. Rennie, J.M., C.F. Hagmann, and N.J. Robertson, Outcome after intrapartum hypoxic ischaemia at term. Semin Fetal Neonatal Med, 2007. 12(5): p. 398-407.

261. Barrett, R.D., et al., Destruction and reconstruction: hypoxia and the developing brain. Birth Defects Res C Embryo Today, 2007. 81(3): p. 163-76.

262. Strackx, E., et al., Fetal asphyxia leads to a decrease in dorsal raphe serotonergic neurons. Developmental Neuroscience, 2008. in press.

263. Rutten, B.P., et al., Age-related loss of synaptophysin immunoreactive presynaptic boutons within the hippocampus of APP751SL, PS1M146L, and APP751SL/PS1M 146L transgenic mice. Am J Pathol, 2005. 167(1): p. 161-73.

264. Rutten, B.P., et al., No alterations of hippocampal neuronal number and synaptic bouton number in a transgenic mouse model expressing the betacleaved C-terminal APP fragment. Neurobiol Dis, 2003. 12(2): p. 110-20.

265. Horner, C.H., H.A. Davies, and M.G. Stewart, Hippocampal synaptic density and glutamate immunoreactivity following transient cerebral ischaemia in the chick. Eur J Neurosci, 1998. 10(12): p. 3913-7.

266. Stepanov, S.S., et al., An ultrastructural study into the effect of global transient cerebral ischaemia on the synaptic population of the cerebellar cortex in rats. Resuscitation, 1998. 39(1-2): p. 99-106.

267. Ramirez, M.R., et al., Neonatal hypoxia-ischemia reduces ganglioside, phos- 
pholipid and cholesterol contents in the rat hippocampus. Neurosci Res, 2003. 46(3): p. 339-47.

268. Wong, T.P., et al., Synaptic numbers across cortical laminae and cognitive performance of the rat during ageing. Neuroscience, 1998. 84(2): p. 403-12. 269. Smith, T.D., et al., Circuit-specific alterations in hippocampal synaptophysin immunoreactivity predict spatial learning impairment in aged rats. J Neurosci, 2000. 20(17): p. 6587-93.

270. George, S., et al., Induced cerebral hypothermia reduces post-hypoxic loss of phenotypic striatal neurons in preterm fetal sheep. Exp Neurol, 2007. 203(1): p. 137-47.

271. Mallard, E.C., et al., Repeated asphyxia causes loss of striatal projection neurons in the fetal sheep brain. Neuroscience, 1995. 65(3): p. 827-36.

272. Mallard, E.C., et al., Repeated episodes of umbilical cord occlusion in fetal sheep lead to preferential damage to the striatum and sensitize the heart to further insults. Pediatr Res, 1995. 37(6): p. 707-13.

273. Goni-de-Cerio, F., et al., Early cell death in the brain of fetal preterm lambs after hypoxic-ischemic injury. Brain Res, 2007. 1151: p. 161-71.

274. Van de Berg, W.D., et al., Impact of perinatal asphyxia on the GABAergic and locomotor system. Neuroscience, 2003. 117(1): p. 83-96.

275. Temel, Y., et al., The functional role of the subthalamic nucleus in cognitive and limbic circuits. Prog Neurobiol, 2005. 76(6): p. 393-413.

276. Fiala, J.C., J. Spacek, and K.M. Harris, Dendritic spine pathology: cause or consequence of neurological disorders? Brain Res Brain Res Rev, 2002. 39(1): p. 29-54.

277. Stroemer, R.P., T.A. Kent, and C.E. Hulsebosch, Increase in synaptophysin immunoreactivity following cortical infarction. Neurosci Lett, 1992. 147(1): p. 21-4.

278. Rosell, A. and J.M. Gimenez-Amaya, Anatomical re-evaluation of the corticostriatal projections to the caudate nucleus: a retrograde labeling study in the cat. Neurosci Res, 1999. 34(4): p. 257-69.

279. Tanaka, D., Jr., Differential laminar distribution of corticostriatal neurons in the prefrontal and pericruciate gyri of the dog. J Neurosci, 1987. 7(12): p. 4095-106.

280. Thomson, A.M. and A.P. Bannister, Interlaminar connections in the neocortex. Cereb Cortex, 2003. 13(1): p. 5-14.

281. Horn, D., N. Levy, and E. Ruppin, Neuronal-based synaptic compensation: 
a computational study in Alzheimer's disease. Neural Comput, 1996. 8(6): p. 1227-43.

282. Sugahara, M. and H. Shiraishi, Synaptic density of the prefrontal cortex regulated by dopamine instead of serotonin in rats. Brain Res, 1998. 814(1-2): p. 143-56.

283. Uyanikgil, Y., et al., Beneficial effects of melatonin on morphological changes in postnatal cerebellar tissue owing to epileptiform activity during pregnancy in rats: light and immunohistochemical study. Brain Res Dev Brain Res, 2005. 159(2): p. 79-86.

284. Lawn, J.E., et al., Why are 4 million newborn babies dying each year? Lancet, 2004. 364(9432): p. 399-401.

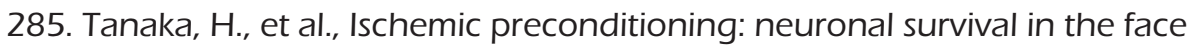
of caspase-3 activation. J Neurosci, 2004. 24(11): p. 2750-9.

286. Stagliano, N.E., et al., Focal ischemic preconditioning induces rapid tolerance to middle cerebral artery occlusion in mice. J Cereb Blood Flow Metab, 1999. 19(7): p. 757-61.

287. Plaschke, K., et al., Permanent cerebral hypoperfusion: 'preconditioninglike' effects on rat energy metabolism towards acute systemic hypotension. Brain Res, 2000. 858(2): p. 363-70.

288. Cogolludo, A., et al., Activation of neutral sphingomyelinase is involved in acute hypoxic pulmonary vasoconstriction. Cardiovasc Res, 2008.

289. Kantor, O., et al., Selective striatal neuron loss and alterations in behavior correlate with impaired striatal function in Huntington's disease transgenic rats. Neurobiol Dis, 2006. 22(3): p. 538-47.

290. Paxinos, G. and C. Watson, The rat brain in stereotaxic coordinates. 1982, San Diego: Academic press.

291. Hoehn, T., et al., Therapeutic hypothermia in neonates. Review of current clinical data, ILCOR recommendations and suggestions for implementation in neonatal intensive care units. Resuscitation, 2008. 78(1): p. 7-12.

292. Dorrepaal, C.A., et al., Inhibition of nitric oxide synthesis following severe hypoxia-ischemia restores autoregulation of cerebral blood flow in newborn lambs. Early Hum Dev, 2001. 60(3): p. 159-170.

293. Lou, H.C., N.A. Lassen, and B. Friis-Hansen, Impaired autoregulation of cerebral blood flow in the distressed newborn infant. J Pediatr, 1979. 94(1): p. 118-21.

294. Williams, J.M., et al., Chronic hypoxic decreases in soluble guanylate cy- 
clase protein and enzyme activity are age dependent in fetal and adult ovine carotid arteries. J Appl Physiol, 2006. 100(6): p. 1857-66.

295. Yoshida, T. and G.K. Owens, Molecular determinants of vascular smooth muscle cell diversity. Circ Res, 2005. 96(3): p. 280-91.

296. Hutanu, C., et al., Vascular development in early ovine gestation: carotid smooth muscle function, phenotype, and biochemical markers. Am J Physiol Regul Integr Comp Physiol, 2007. 293(1): p. R323-33.

297. Rzucidlo, E.M., K.A. Martin, and R.J. Powell, Regulation of vascular smooth muscle cell differentiation. J Vasc Surg, 2007. 45 Suppl A: p. A25-32.

298. Su, C., et al., Development of neuroeffector mechanisms in the carotid artery of the fetal lamb. Blood Vessels, 1977. 14(1): p. 12-24.

299. Longo, L.D., et al., Developmental changes in alpha 1-adrenergic receptors, IP3 responses, and NE-induced contraction in cerebral arteries. Am J Physiol, 1996. 271(6 Pt 2): p. H2313-9.

300. Campbell, M.E., et al., Maternal nutrient restriction reduces carotid artery constriction without increasing nitric oxide synthesis in the late gestation rat fetus. Pediatr Res, 2005. 58(5): p. 840-4.

301. Williams, S.J., et al., Differential effects of maternal hypoxia or nutrient restriction on carotid and femoral vascular function in neonatal rats. Am J Physiol Regul Integr Comp Physiol, 2005. 288(2): p. R360-7.

302. Fletcher, A.J., et al., Development of the ovine fetal cardiovascular defense to hypoxemia towards full term. Am J Physiol Heart Circ Physiol, 2006. 291(6): p. H3023-34.

303. Beckman, J.S., The double-edged role of nitric oxide in brain function and superoxide-mediated injury. J Dev Physiol, 1991. 15(1): p. 53-9.

304. Clavier, N., et al., Effect of postischemic hypoperfusion on vasodilatory mechanisms in cats. Am J Physiol, 1994. 267(5 Pt 2): p. H2012-8.

305. Clavier, N., et al., Cerebral blood flow is reduced by $\mathrm{N}$ omega-nitro-Larginine methyl ester during delayed hypoperfusion in cats. Am J Physiol, 1994. 267(1 Pt 2): p. H174-81.

306. Dorrepaal, C.A., et al., Effect of post-hypoxic-ischemic inhibition of nitric oxide synthesis on cerebral blood flow, metabolism and electrocortical brain activity in newborn lambs. Biol Neonate, 1997. 72(4): p. 216-26.

307. Greenberg, R.S., et al., Effect of nitric oxide synthase inhibition on postischemic cerebral hyperemia. Am J Physiol, 1995. 269(1 Pt 2): p. H34 1-7.

308. Sato, S., et al., Electron paramagnetic resonance study on nitric oxide pro- 
duction during brain focal ischemia and reperfusion in the rat. Brain Res, 1994. 647(1): p. 91-6.

309. Perlman, J.M., Intervention strategies for neonatal hypoxic-ischemic cerebral injury. Clin Ther, 2006. 28(9): p. 1353-65.

310. Kapinya, K.J., K. Prass, and U. Dirnagl, Isoflurane induced prolonged protection against cerebral ischemia in mice: a redox sensitive mechanism? Neuroreport, 2002. 13(11): p. 1431-5.

311. Kersten, J.R., et al., Isoflurane mimics ischemic preconditioning via activation of K(ATP) channels: reduction of myocardial infarct size with an acute memory phase. Anesthesiology, 1997. 87(2): p. 361-70.

312. Sarkar, S., et al., Effects of therapeutic hypothermia on multiorgan dysfunction in asphyxiated newborns: whole-body cooling versus selective head cooling. J Perinatol, 2009.

313. Benjelloun, N., et al., Inflammatory responses in the cerebral cortex after ischemia in the P7 neonatal Rat. Stroke, 1999. 30(9): p. 1916-23; discussion 1923-4.

314. Ezquer, M.E., S.R. Valdez, and A.M. Seltzer, Inflammatory responses of the substantia nigra after acute hypoxia in neonatal rats. Exp Neurol, 2006. 197(2): p. 391-8.

315. Lu, X., et al., Activation of NF-\{kappa\}B is a critical element in the antiapoptotic effect of anesthetic preconditioning. Am J Physiol Heart Circ Physiol, 2009.

316. Gustavsson, M., et al., Global gene expression in the developing rat brain after hypoxic preconditioning: involvement of apoptotic mechanisms? Pediatr Res, 2007. 61(4): p. 444-50.

317. Gidday, J.M., et al., Nitric oxide mediates cerebral ischemic tolerance in a neonatal rat model of hypoxic preconditioning. J Cereb Blood Flow Metab, 1999. 19(3): p. 331-40.

318. Lawson, C.S., D.J. Coltart, and D.J. Hearse, "Dose"-dependency and temporal characteristics of protection by ischaemic preconditioning against ischaemia-induced arrhythmias in rat hearts. J Mol Cell Cardiol, 1993. 25(12): p. 1391-402.

319. Li, Y.W., P. Whittaker, and R.A. Kloner, The transient nature of the effect of ischemic preconditioning on myocardial infarct size and ventricular arrhythmia. Am Heart J, 1992. 123(2): p. 346-53.

320. Baxter, G.F., F.M. Goma, and D.M. Yellon, Characterisation of the infarct- 
limiting effect of delayed preconditioning: timecourse and dose-dependency studies in rabbit myocardium. Basic Res Cardiol, 1997. 92(3): p. 159-67.

321. Obrenovitch, T.P., Molecular physiology of preconditioning-induced brain tolerance to ischemia. Physiol Rev, 2008. 88(1): p. 211-47.

322. Perez-Pinzon, M.A. and J.G. Born, Rapid preconditioning neuroprotection following anoxia in hippocampal slices: role of the K+ ATP channel and protein kinase C. Neuroscience, 1999. 89(2): p. 453-9.

323. Cimarosti, $H_{\text {., }}$ et al., Hypoxic preconditioning in neonatal rat brain involves regulation of excitatory amino acid transporter 2 and estrogen receptor alpha. Neurosci Lett, 2005. 385(1): p. 52-7.

324. Barker, D.J., The origins of the developmental origins theory. J Intern Med, 2007. 261(5): p. 412-7.

325. Thompson, C., et al., Birth weight and the risk of depressive disorder in late life. Br J Psychiatry, 2001. 179: p. 450-5.

326. Nilsson, E., et al., Fetal growth restriction and schizophrenia: a Swedish twin study. Twin Res Hum Genet, 2005. 8(4): p. 402-8.

327. Breslau, N., et al., Psychiatric sequelae of low birth weight at 6 years of age. J Abnorm Child Psychol, 1996. 24(3): p. 385-400.

328. Breslau, N., et al., Low birth weight and neurocognitive status at six years of age. Biol Psychiatry, 1996. 40(5): p. 389-97.

329. Breslau, N. and H.D. Chilcoat, Psychiatric sequelae of low birth weight at 11 years of age. Biol Psychiatry, 2000. 47(11): p. 1005-11.

330. Johnson, E.O. and N. Breslau, Increased risk of learning disabilities in low birth weight boys at age 11 years. Biol Psychiatry, 2000. 47(6): p. 490-500.

331. Strackx, E., et al., Fetal asphyxia leads to the loss of striatal presynaptic boutons in adult rats. Int J Dev Neurosci, 2009.

332. Hagberg, H., et al., Preconditioning and the developing brain. Semin Perinatol, 2004. 28(6): p. 389-95.

333. Strackx, E., et al., Alterations of brain apoptotic cell death and carotid artery reactivity in a fetal asphyctic preconditioning model. Frontiers in Bioscience, 2009. In press.

334. Boksa, P., D. Wilson, and J. Rochford, Responses to stress and novelty in adult rats born vaginally, by cesarean section or by cesarean section with acute anoxia. Biol Neonate, 1998. 74(1): p. 48-59.

335. Bjelke, B., et al., Asphyctic lesion: proliferation of tyrosine hydroxylase-immunoreactive nerve cell bodies in the rat substantia nigra and functional 
changes in dopamine neurotransmission. Brain Res, 1991. 543(1): p. 1-9.

336. Rutten, K., et al., Sub-chronic rolipram treatment leads to a persistent improvement in long-term object memory in rats. Neurobiol Learn Mem, 2008. 90(3): p. 569-75.

337. Shepherd, J.K., et al., Behavioural and pharmacological characterisation of the elevated "zero-maze" as an animal model of anxiety. Psychopharmacology (Berl), 1994. 116(1): p. 56-64.

338. Van den Hove, D.L., et al., Prenatal restraint stress and long-term affective consequences. Dev Neurosci, 2005. 27(5): p. 313-20.

339. Porsolt, R.D., et al., Behavioural despair in rats: a new model sensitive to antidepressant treatments. Eur J Pharmacol, 1978. 47(4): p. 379-91.

340. Sulon, J., et al., Radioimmunoassay for aldosterone and deoxycorticosterone in human plasma. Comparison of various antisera and determination of normal values. Clin Chim Acta, 1976. 72(3): p. 275-84.

341. Wu, X., et al., Hypoxic preconditioning induces delayed cardioprotection through p38 MAPK-mediated calreticulin upregulation. Shock, 2007. 27(5): p. 572-7.

342. Shi, Y., et al., Mitochondrial big conductance KCa channel and cardioprotection in infant rabbit heart. J Cardiovasc Pharmacol, 2007. 50(5): p. 497-502. 343. McAuliffe, J.J., L. Miles, and C.V. Vorhees, Adult neurological function following neonatal hypoxia-ischemia in a mouse model of the term neonate: water maze performance is dependent on separable cognitive and motor components. Brain Res, 2006. $1118(1)$ : p. 208-21.

344. Lubics, A., et al., Neurological reflexes and early motor behavior in rats subjected to neonatal hypoxic-ischemic injury. Behav Brain Res, 2005. 157(1): p. 157-65.

345. Kiss, P., et al., Effects of perinatal asphyxia on the neurobehavioral and retinal development of newborn rats. Brain Res, 2009. 1255: p. 42-50.

346. Venerosi, A., et al., Acute global anoxia during C-section birth affects dopamine-mediated behavioural responses and reactivity to stress. Behav Brain Res, 2004. 154(1): p. 155-64.

347. Venerosi, A., et al., C-section birth per se or followed by acute global asphyxia altered emotional behaviour in neonate and adult rats. Behav Brain Res, 2006. 168(1): p. 56-63.

348. Wang, L., R.J. Traystman, and S.J. Murphy, Inhalational anesthetics as preconditioning agents in ischemic brain. Curr Opin Pharmacol, 2008. 8(1): p. 104- 
10.

349. El-Khodor, B.F. and P. Boksa, Long-term reciprocal changes in dopamine levels in prefrontal cortex versus nucleus accumbens in rats born by Caesarean section compared to vaginal birth. Exp Neurol, 1997. 145(1): p. 118-29.

350. Vogl, S.E., et al., Mode of delivery is associated with maternal and fetal endocrine stress response. Bjog, 2006. 113(4): p. 441-5.

351. Vaillancourt, C. and P. Boksa, Caesarean section birth with general anesthesia increases dopamine-mediated behavior in the adult rat. Neuroreport, 1998. 9(13): p. 2953-9.

352. Berger, N., C. Vaillancourt, and P. Boksa, Genetic factors modulate effects of C-section birth on dopaminergic function in the rat. Neuroreport, 2000. $11(3)$ : p. 639-43.

353. El-Khodor, B. and P. Boksa, Caesarean section birth produces long term changes in dopamine D1 receptors and in stress-induced regulation of D3 and D4 receptors in the rat brain. Neuropsychopharmacology, 2001. 25(3): p. 42339.

354. Boksa, P., Y. Zhang, and A. Bestawros, Dopamine D1 receptor changes due to caesarean section birth: effects of anesthesia, developmental time course, and functional consequences. Exp Neurol, 2002. 175(2): p. 388-97.

355. Gillman, M.W., et al., Meeting report on the 3rd International Congress on Developmental Origins of Health and Disease (DOHaD). Pediatr Res, 2007. $61(5$ Pt 1): p. 625-9.

356. Gluckman, P.D., et al., Life-long echoes-a critical analysis of the developmental origins of adult disease model. Biol Neonate, 2005. 87(2): p. 127-39.

357. Gluckman, P.D., M.A. Hanson, and C. Pinal, The developmental origins of adult disease. Matern Child Nutr, 2005. 1(3): p. 130-41.

358. Borenstein, A.R., C.I. Copenhaver, and J.A. Mortimer, Early-life risk factors for Alzheimer disease. Alzheimer Dis Assoc Disord, 2006. 20(1): p. 63-72.

359. Landrigan, P.J., et al., Early environmental origins of neurodegenerative disease in later life. Environ Health Perspect, 2005. 113(9): p. 1230-3.

360. Miller, D.B. and J.P. O'Callaghan, Do early-life insults contribute to the latelife development of Parkinson and Alzheimer diseases? Metabolism, 2008. 57 Suppl 2: p. S44-9.

361. Whalley, L.J., F.D. Dick, and G. McNeill, A life-course approach to the aetiology of late-onset dementias. Lancet Neurol, 2006. 5(1): p. 87-96.

362. Gale, C.R. and C.N. Martyn, Birth weight and later risk of depression in a national birth cohort. Br J Psychiatry, 2004. 184: p. 28-33. 


\section{Chapter 1}

In chapter 1, a general introduction was given on the current knowledge of developmental asphyxia and hypoxic-ischemic preconditioning. In addition, the different animal models and the aims of the studies in this thesis were introduced.

\section{Chapter 2}

In chapter 2, we investigated the long-term effects of fetal asphyxia on locomotor activity and the associated dopaminergic circuitry. Severe fetal asphyxia was induced at embryonic day 17 by clamping the uterine and ovarian arteries for 75 minutes, followed by full-term vaginally delivery. At 6 months of age, rats were tested for changes in locomotor activity and tyrosine hydroxylase (TH) was used as a marker for the assessment of the dopaminergic system. Behavioral analysis demonstrated that fetal asphyctic animals showed less basal and amphetamine-induced locomotor activity. This decrease in locomotion was related to a substantial loss of dopaminergic neurons in the substantia nigra pars compacta and a loss of TH-immunoreactivity in the striatum. Further, more GFAP-positive astrocytes were found in both areas. A last finding was that fetal asphyxia also caused enlarged ventricles associated with smaller white matter volumes. In conclusion, this chapter shows that fetal asphyxia can cause long-lasting changes in motor behavior associated with morphological changes in the dopaminergic nigrostriatal circuitry.

\section{Chapter 3}

In chapter 3, the same severe fetal asphyxia model was used as in chapter 1 to investigate its long-term effects on the serotonergic (5-HT) system. At 19 months of age, rats were tested for anxiety-related behavior during an open field test. Fetal asphyctic animals spent significantly less time in the center and more time along the walls, demonstrating increased anxiety-related behavior. Post-mortem analysis of the brains revealed that these behavioral changes 
were associated with an age-related loss of serotonergic cells in the dorsal raphe. In addition, these neurons were smaller in comparison to control animals. Summarized, these results of this chapter support the notion that asphyctic insults during critical periods of brain development could predispose to serotonergic abnormalities and anxiety deficits in adulthood.

\section{Chapter 4}

Chapter 4 is the last chapter that investigates the long-term effects of severe fetal asphyxia. In this chapter, we investigated the effects on synapse number. Synaptophysin was used as a marker for presynaptic boutons. Using image analysis, we found that fetal asphyxia decreased the number and the density of presynaptic boutons in the striatum, while there was an increase in the 5th layer of the frontal cortex. The results of this fourth chapter suggest that fetal asphyxia can have long-lasting effects on synaptic organization that might contribute to a developmental etiology of different neurological disorders and aging.

\section{Chapter 5}

In chapter 5, the concept of fetal asphyctic preconditioning was introduced for the first time. A fetal asphyctic insult (FA) was induced, as a preconditioning stimulus, by clamping the uterine vasculature for 30 minutes on embryonic day 17 (E17, first hit). At birth (PO), severe perinatal asphyxia (SPA) was induced by placing the uterine horns, still containing the fetuses, in a water bath for 19 minutes. Using a TUNEL-staining cell death was examined in the striatum and the prefrontal cortex. Results showed that the preconditioned animals showed significantly less cell death in both areas compared to non-preconditioned animals. Therefore, this study shows the first evidence that this model offers new possibilities to study global fetal asphyctic preconditioning. However, additional experiments with extra control groups are necessary.

\section{Chapter 6}

In chapter 6, the new fetal asphyctic preconditioning model was further vali- 
dated. Additional control groups and brain regions were analysed. The results confirmed the findings of the 5th chapter. Preconditioning lowered cell death in the striatum, prefrontal cortex and hippocampus in comparison to non-preconditioned animals. In addition, the preconditioned animals also showed a decrease in the number of astrocytes. Furtermore, the extra control groups showed that there were no significant changes due to the experimental procedures per se. Taken together, we can conclude that fetal asphyctic preconditioning causes a decrease in cell death and astrogliosis. Consequently, mild FA might cause neuroprotection by inducing anti-apoptotic mechanisms and by attenuating astrogliosis.

\section{Chapter 7}

The aim of this last experimental chapter was to investigate if fetal asphyctic preconditioning can, in addition to a histological neuroprotection, also provide the long-lasting behavioral protection. For that reason, the functional outcome was assessed at 6 months of age using different behavioral tests: the open field for locomotor activity, the elevated zero maze for anxiety-related behavior, the forced swim test for depression-related behavior and the object recognition task for cognition. Locomotor activity in the open field test and cognitive performance in the object recognition task were impaired in the nonpreconditioned animals compared to the control groups. Most importantly, fetal asphyctic preconditioning was able to preserve both locomotor activity and cognition function. In conclusion, fetal asphytcic preconditioning induces a long-lasting, functional protection against severe perinatal asphyxia.

\section{Chapter 8}

\section{Fetal asphyxia: Friend or foe?}

In this last chapter the main findings of this thesis are discussed and an attempt is made to put these findings in a clinical perspective. In conclusion, the present work stresses that every coin has two sides. While severe fetal asphyxia is a serious threat to development, causing both short and long-term functional and morphological impairments in the rat brain, mild fetal asphyxia can induce 
a long-lasting neuroprotection. The question, however, whether the knowledge about fetal asphyctic preconditioning can contribute to a cure of neonatal encephalopathy remains yet unsolved. 


\section{Vereenvoudigde samenvatting}

Een tekort aan bloedstroming en zuurstof (asfyxie) voor, tijdens of vlak na de geboorte is wereldwijd nog steeds één van de meest voorkomende problemen in de verloskunde en de neonatologie. Het vormt één van de voornaamste oorzaken van mortaliteit bij pasgeborenen, aangezien het ongeveer 23\% van alle neonatale sterfgevallen veroorzaakt. Het ontstaan van asfyxie ligt meestal in een gebrekkige zuurstofoverdracht van moeder naar kind. De meest frequente oorzaken hiervan zijn een loslatende placenta, een afklemming van de navelstreng of een moeilijke bevalling. Op deze manier krijgt het kind uiteindelijk te weinig zuurstof in het bloed en de vitale organen, met hersenschade als mogelijk gevolg. Hersenbeschadiging door asfyxie komt voor bij ongeveer 1 per 1000 van alle voldragen pasgeborenen. Afhankelijk van de duur en de ernst van het zuurstofgebrek kunnen deze structurele beschadigingen leiden tot de ontwikkeling van verschillende aandoeningen, zoals een mentale achterstand, leerachterstand, epilepsie en/of motorische stoornisssen. Een onherstelbaar hersenletsel is een tragedie voor het kind en de familie en het brengt belangrijke medische, sociale en economische implicaties met zich mee.

Over de lange termijn effecten van asfyctische hersenschade, bijvoorbeeld tijdens de adolescentie of op volwassen leeftijd, is echter weinig of niets bekend. Mogelijk zou het een belangrijke factor kunnen zijn die bijdraagt aan neurodegeneratieve ziekten, zoals versnelde verouderingsprocessen bij bijvoorbeeld de ziekte van Parkinson of de ziekte van Alzheimer.

Hoewel er volop onderzoek gebeurt, zijn er momenteel maar weinig efficiënte therapieën voorhanden. Tot voor kort bestond de behandeling enkel uit het bestrijden van de voornaamste symptomen, zoals controleren van de bloeddruk, het behandelen van epileptische aanvallen en de ademhaling ondersteunen. De laatste jaren werd echter een groot aantal onderzoeken verricht naar effectievere behandelings- en preventiemethoden. Uit deze resultaten is gebleken dat bij voldragen pasgeborenen koeling van het hoofd en/of van het hele lichaam de kans op de ontwikkeling van neurologische aandoeningen vermindert. Deze behandelingsstrategie is echter enkel effectief gebleken bij matige asfyxie. Er is dus een dringende nood aan nieuwe en betere behan- 
delingsmethoden.

Experimentele dierenmodellen, zoals in deze thesis beschreven worden, zijn dan ook een waardevol hulpmiddel om de - nog onbekende - lange termijn gevolgen van asfyxie en nieuwe behandelingsmethoden te onderzoeken.

De onderzoeksvraag in deze thesis bestaat uit 2 delen. De eerste hoofdvraag heeft als doel de voornaamste lange termijn gevolgen van asfyxie vóór de geboorte te karakteriseren. Dit komt dan ook aan bod in hoofdstukken 2 tot en met 4. De tweede hoofdvraag van de thesis richt zich meer op beschermende mechanismen. De hoofdstukken 5 tot en met 7 concentreren zich op de ontwikkeling van een nieuw dierenmodel voor asfyctische preconditionering. Preconditionering is het fenomeen waarbij een milde asfyxie het weefsel resistenter maakt tegen de schadelijke effecten van een langdurige asfyxie. Hoewel er inmiddels al behoorlijk wat onderzoek verricht is naar asfyctische preconditionering, zijn de exacte mechanismen nog steeds onbekend.

Met betrekking tot de eerste onderzoeksvraag, werd in hoofdstuk 2 onderzocht of ernstige asfyxie tijdens de prenatale periode kan leiden tot veranderingen in motoractiviteit en de gerelateerde hersenstructuren op volwassen leeftijd. Ernstige foetale asfyxie werd geïnduceerd in een zwangere rat door de bloedvaten naar de baarmoeder en de ovaria af te klemmen voor 75 minuten op dag 17 van de zwangerschap. Uit dit onderzoek is gebleken dat een dergelijke foetale asfyxie leidt tot verminderde motoractiviteit op de volwassen leeftijd van 6 maanden. Dit was geassocieerd met een daling van het aantal dopaminerge neuronen in de substantia nigra, dewelke betrokken zijn bij het initiëren van beweging. Daarnaast werden in dit proefschrift ook de effecten van foetale asfyxie op angstgedrag en de gerelateerde hersengebieden bestudeerd (hoofdstuk 3). Met deze studie werd aangetoond dat foetale asfyxie een verhoogd angstgedrag veroorzaakt op een leeftijd van 19 maanden. Deze gedragsverandering was gerelateerd aan verlies van serotonerge neuronen in de dorsale raphe, een hersenkern belangrijk voor angst- en depressiegerelateerd gedrag. In een laatse studie met dit dierenmodel (hoofdstuk 4), werd gekeken naar het aantal verbindingen tussen hersencellen, ook wel synapsen genoemd. Deze verbindingen zijn belangrijk voor de communicatie tussen verschillende cellen. Er kwamen veranderingen naar voren in twee verschillende hersengebieden. In het striatum werd een daling van het aantal 
synapsen waargenomen, terwijl in de prefrontale cortex een stijging werd gevonden. Samenvattend zouden we uit deze bevindingen kunnen concluderen dat ernstige foetale asphyxie verstrekkende en langdurige gevolgen heeft. Verder leveren deze studies het eerste bewijs dat foetale asfyxie mogelijk een bijdragende factor is voor de ontwikkeling van verschillende neurodegeneratieve ziekten, zoals de ziekte van Parkinson. Om die reden zouden deze bevindingen belangrijke implicaties kunnen hebben voor het verklaren van het ontstaan van bepaalde neurologische aandoeningen.

Zoals hierboven reeds werd aangehaald, handelt de tweede onderzoeksvraag in dit proefschrift over asfyctische preconditionering. In de volgende hoofdstukken werd een nieuw rattenmodel geïntroduceerd. Een milde foetale asfyxie werd geïnduceerd, als een preconditioneringsstimulus, door de bloedvaten naar de baarmoeder en ovaria af te klemmen voor slechts 30 minuten (in tegenstelling tot 75 minuten in de eerste hoofdstukken) op dag 17 van de zwangerschap. Vier dagen later, op de dag van de geboorte, werd een ernstige perinatale asfyxie teweeggebracht door de baarmoeder met de rattenpupjes onder te dompelen in een waterbad gedurende 20 minuten. De hypothese is dat milde foetale asfyxie (=preconditionering) beschermt tegen hersenbeschadiging veroorzaakt door ernstige perinatale asfyxie. In hoofdstuk 5 en hoofdstuk 6 werd naar voren gebracht dat de gepreconditioneerde dieren, zoals verwacht, minder celdood (apoptose) vertoonden dan de nietgepreconditioneerde dieren 7 dagen na de geboorte in alle onderzochte hersengebieden, waaronder de hippocampus, de cortex en het striatum. Verder hadden de gepreconditioneerde dieren minder gliose dan de nietgepreconditioneerde dieren. Gliose is een reactie van het hersenweefsel op een schadelijke stimulus, waarbij de steuncellen in de hersenen, ook wel astrocyten genaamd, vermeerderen. Preconditionering beschermt het brein dus tegen acute hersenschade. De langdurige, gedragsmatige effecten van asfyctische preconditionering werden geanalyseerd in hoofdstuk 7. Ernstige perinatale asfyxie veroorzaakte problemen met zowel spontane motoractiviteit als leren en geheugen. Deze problemen werden volledig tenietgedaan door preconditionering. Uit deze studies kunnen we besluiten dat milde foetale asfyxie, als een preconditioningsstimulus, waarschijnlijk een langdurige bescherming biedt tegen asfyctische hersenbeschadiging en de geassocieerde gedragsmatige problemen. Deze bevindingen zouden mogelijk belangrijke klinische 
implicaties kunnen hebben. Om ethische redenen, is preconditionering van de hersenen echter niet klinisch toepasbaar bij patiënten. Het doel van dit onderzoek is echter om dezelfde of gelijkaardige beschermende mechanismen na te bootsen via bijvoorbeeld farmacologische interventies. Verder experimenteel en klinisch onderzoek is nodig om na te gaan of deze potentiële beschemingsmechanismen ook in de klinische praktijk toepasbaar zijn.

\section{Slotopmerking}

Foetale asfyxie: Vriend of vijand?

Dit proefschrift toont aan dat de effecten van foetale asfyxie tweeledig kunnen zijn. In het eerste deel werd aangetoond dat ernstige foetale asfyxie een gevaarlijke bedreiging kan vormen voor de ontwikkeling (vijand). Zo leidt ernstige foetale asfyxie tot een stoornis van de motoractiviteit en angstgedrag, wat gepaard gaat met het verlies van respectievelijk dopaminerge en serotonerge neuronen. Ook het aantal verbindingen tussen hersencellen verandert onder invloed van ernstige asfyxie. In tegenstelling tot deze schadelijke bevindingen, laat het tweede deel van het proefschrift zien dat asfyxie, wanneer deze voldoende mild is, ook een beschermende werking kan bieden (vriend). Verder onderzoek zal echter moeten uitwijzen of deze beschermende mechanismen mogelijk kunnen bijdragen aan de ontwikkeling van een nieuwe geneeswijze. 


\section{Dankwoord}

Aan het begin lijkt een promotie-onderzoek een lange tijd, maar voor je het weet zit je te knoeien met de teksten en de indeling van je proefschift. Dit alles zou onmogelijk zijn zonder de hulp en de inspiratie van anderen. Daarom zou ik van deze laatste bladzijden gebruik willen maken om hen allemaal te bedanken.

Allereerst zou ik graag mijn promotoren, Prof. dr. Hans Vles en Prof. dr. Luc Zimmermann, bedanken om mij de mogelijkheid te geven een promotie-onderzoek te starten. Jullie hebben me de kans gegeven om mezelf verder te ontwikkelen zowel op wetenschappelijk als op persoonlijk vlak. Bedankt voor de fijne samenwerking en het in mij gestelde vertrouwen.

Beste Danilo, copromotor, met jou is dit promotietraject begonnen, waarvan dit proefschrift de wetenschappelijke kroon is. Je hebt me veel vrijheid en verantwoordelijkheid gegeven, hetgeen ik zeer heb gewaardeerd. Heel erg bedankt voor je hulp, tijd en 'altijd-even-positieve feedback'. Je bekeek mijn onderzoeksresulaten vanuit een ander, meer klinisch, perspectief en dat heeft me ontzettend geholpen. Deze link tussen fundamenteel wetenschappelijk onderzoek en de kliniek was voor mij een belangrijke motivator. Ik hoop dat we deze prettige samenwerking nog lang mogen verder zetten.

Daniël, jammer dat je op het laatste moment dan toch geen copromotor kon zijn. Voor mij had je dat meer dan verdiend. In elk geval bedankt voor je kritisch wetenschappelijke blik op de experimenten en op dit proefschrift. Het was motiverend om met je van gedachten te wisselen. Ik hoop dan ook dat ik in de toekomst zo nu en dan toch nog eens jouw expertise mag raadplegen.

Prof. dr. Harry Steinbusch als hoofd van de School of Mental Health and Neurosciende, heeft u mijn onderzoek altijd vanop afstand gevolgd. $U$ hield mijn vorderingen nauwlettend in het oog en u was altijd bereikbaar voor de nodige discussies. Prof. dr. Carlos Blanco, ook u hield mijn vooruitgang en manuscripten vanop een afstand in het oog. Bedankt voor jullie inzet! 
Mijn paranimfen, Marijke en Rinske, altijd goed om jullie in de buurt te hebben. Marijke, het zal voor niemand een verrasssing zijn dat ik jou gekozen heb als paranimf. We hebben immers samen al veel leuke en minder leuke dingen meegemaakt, ook voor onze tijd als AIO. Denk bijvoorbeeld maar aan onze leuke 'Diepenbeek-tijd' en ons tripje naar New York. Bedankt voor alles en heel veel succes met het afronden van jouw promotie. Rinske, mijn andere paranimf, ook jou ken ik al van op de MLW schoolbanken, hoewel ik je later pas echt heb leren kennen. Ook jij bedankt voor de super mooie tijd. Samen feestjes organiseren op het lab en samen acrobatieken zou ik voor geen geld in de wereld willen missen. Ook jij heel veel succes met het afronden van jouw promotie-onderzoek. Meisjes, ik vind het een grote eer dat jullie op deze dag beide langs me staan.

Plezier hebben in je werk is ook voor een groot gedeelte afhankelijk van je werkomgeving. Daarom zijn natuurlijk ook de collega's belangrijk met wie je altijd kon overleggen, tips kon krijgen en waar je uiteraard ook je blijdschap en frustraties kwijt kon. Zonder jullie waren de voorbije 3 jaar niet hetzelfde geweest. Dames, Annerieke, Eva B, Eva vD, Evi, Imke, Kathleen, Kim, Marijke, Marisela en Rinske, bedankt voor alle mooie momenten in de Efteling, op de skipiste, tijdens het stappen, tijdens de koffiepauzes of gewoon in het labo. Bij deze zou ik ook graag alle andere collega's van de afdelingen basale neurowetenschappen en kindergeneeskunde willen bedanken zonder hen allemaal bij naam te noemen! In het bijzonder zou ik ook de analysten (Hellen, Marjanne, Denise, Jorike, Marjan en Eline) en de mensen van het secretariaat (Marie-Therèse, Anouk en Akke) willen vermelden voor al het harde werk. Natuurlijk mag ik de CPV-ers niet vergeten. Ook jullie hebben allen een steentje bijgedragen aan dit promotietraject. Tot slot, zou ik ook mijn opvolgsters, Evi en Kimberly, heel veel succes willen wensen met hun promotie-onderzoek! Hopelijk volgen er nog enkele mooie en productieve jaren samen.

En, gelukkig bestaat er ook meer dan alleen onderzoek in het leven, ook al lijkt dat niet altijd zo. Promoveren kan niet zonder afwisseling, ontspanning en afleiding. Naast de collega's zijn er dan ook veel mensen die me, op meer en minder cruciale momenten, terzijde hebben gestaan. Mijn schoonfamilie, Mieke, Jaak, Nele, Michael, Lieve, Willem, Jan en Ana. Jullie hebben de vorderingen van mijn onderzoek en dit boekje altijd met belangstelling gevolgd. Dit ver- 
dient een groot applaus. Krijn, oma en opa dit geldt uiteraard ook voor jullie. Kristine, lieve zus, jij ook bedankt voor alles! Zorg goed voor mijn metekindje!

Liefste papa en mama, ook jullie mag ik zeker niet vergeten. Promoveren daar kies je zelf voor en natuurlijk moet je dat zelf doen, maar het feit dat alle mogelijkheden door jullie werden geboden om mezelf te ontwikkelen, zoals als ik dat wilde, is veel meer dan enkel een bedankje waard. Door jullie onvoorwaardelijke liefde, steun en vertrouwen, in welk opzicht dan ook, heb ik me kunnen ontwikkelen tot de persoon die ik nu ben... en dat is meer dan een gepromoveerd onderzoeker alleen. Dikke kus en bedankt!

Ward, lieve schat, het feit dat ik altijd op jou kan rekenen, is heel veel voor me waard. Ik zie je graag! Bedankt voor alles dat was, is en nog komen zal...

Tenslotte; ik weet dat velen deze tekst als eerste lezen, en zich dan stiekem afvragen: 'Word ik genoemd?'. Mezelf kennende, vergeet ik waarschijnlijk wel mensen die ik niet had willen en mogen vergeten. Dat je dit nu leest, maakt de kans groot dat je één van die mensen bent. Daarom, bij deze, ook voor jou: Bedankt!

Eveline

Hasselt, januari 2010 


\section{Curriculum vitae}

Eveline Strackx werd geboren op 10 oktober 1984 in Neerpelt (België). In 2002 voltooide zij haar middelbare opleiding wetenschappen-wiskunde aan het 'Koninklijk Atheneum' in Overpelt. In datzelfde jaar startte ze haar studie biomedische wetenschappen aan het vroegere 'Limburgs Universitair Centrum' in Diepenbeek (nu Universiteit Hasselt). Haar masterstage liep ze op de afdeling 'Psychiatrie en Neuropsychologie' aan de Universiteit van Maastricht (UM). In 2006 behaalde ze dan ook haar masterdiploma in de afstudeerrichting 'Klinische Moleculaire Levenswetenschappen'. Twee maanden later startte ze als promovenda aan de 'School of Mental Health and Neuroscience' (MheNS) aan de UM in samenwerking met de afdeling kindergeneeskunde en de 'European Graduate School of Neuroscience' (EURON) onder de supervisie van Prof. Vles, Prof. Zimmermann, dr. Gavilanes and dr. Van den hove. Tijdens dit 3-jarig traject onderzocht ze de effecten van foetale asfyxie en foetale asfyctische preconditionering tijdens vroege periodes van de hersenontwikkeling. De resultaten van haar onderzoek worden beschreven in dit proefschrift. Verder ontving ze recent een Kootstra Fellowship, een beurs voor talentvolle aspirant postdocs van de UM.

Eveline Strackx was born on October 10th 1984 in Neerpelt (Belgium). In 2002 she graduated from secondary school at the 'Koninklijk Atheneum' in Overpelt. Later that year, she started her bachelor Biomedical Sciences at the former Limburg University Center at Diepenbeek. She did her master internship at the department of Psychiatry en Neuropsychology at the Maastricht University(UM). In 2006 she graduated as a master in Clinical Molecular Lifesciences. Two months later, she started her PhD-project at the School of Mental Health and Neuroscience (MheNS) at the UM in collaboration with the department of pediatrics and the European Graduate School of Neuroscience (EURON) under the supervision of Prof. Vles, Prof. Zimmermann, dr. Gavilanes and dr. Van den Hove. During this 3-year-during period, she investigated the effects of fetal asphyxia and fetal asphyctic preconditioning during early periods of brain development. The results of her PhD-projects are presented in this thesis. Furthermore, she was recently awarded a Kootstra Fellowship for talented aspirant postdocs by the UM. 


\section{List of publications}

\section{Peer-reviewed publications}

E. Strackx, D.L.A. Van den Hove, H.P. Steinbusch, H.W.M. Steinbusch, J.S.H Vles, C.E. Blanco and A.W.D. Gavilanes (2008). A combined behavioral and morphological study on the effects of fetal asphyxia on the nigrostriatal dopaminergic system in the adult rat. Experimental Neurology, 211 (2): 413-422.

E. Strackx, D.L.A. Van den Hove, H.P. Steinbusch, J. Prickaerts, J.S.H. Vles, C.E. Blanco, H.W.M. Steinbusch and A.W.D. Gavilanes (2008). Fetal asphyxia leads to a decrease in dorsal raphe serotonergic neurons. Developmental Neuroscience 30(5); 358-366.

A.W.D. Gavilanes, E. Strackx (joint first), B.W. Kramer, M. Gantert, D.L.A. Van den Hove, H.P. Steinbusch, Y. Garnier, E. Cornips, H.W.M. Steinbusch, L.J. Zimmermann and J.S.H. Vles (2009). Chorioamnionitis induced by intraamniotic lipopolysaccharide resulted in an interval-dependent increase in central nervous system injury in the fetal sheep. American Journal of Obstetrics and Gynecology, 200(4); 437.e1-8.

E. Strackx, B. Zoer, D.L.A. Van den Hove, H.P. Steinbusch, H.W.M. Steinbusch, C.E. Blanco, J.S.H. Vles, E. Villamor and A.W.D. Gavilanes (In press). Alterations of brain apoptotic cell death and carotid artery reactivity in a fetal asphyxia preconditioning model. Frontiers in Bioscience.

A.W.D. Gavilanes, M. Gantert, E. Strackx, L.J. Zimmermann, S. Seeldrayers, J.S.H. Vles and B.W. Kramer (In press). Increased intrapartal EEG delta frequency corresponds to CNS injury caused by chorioamnionitis in the fetal sheep. Frontiers in Bioscience. 
E. Strackx, D.L.A. Van den Hove, H.P. Steinbusch, H.W.M. Steinbusch, J.S.H. Vles, C.E. Blanco and A.W.D. Gavilanes (in press). Fetal asphyxia leads to a loss of striatal presynaptic boutons in adult rats. International Journal of Developmental Neuroscience.

E. Strackx, A.W.D. Gavilanes, D.L.A. Van den Hove, j. Prickaerts, L.J. Zimmermann, H.W.M. Steinbusch, C.E. Blanco and J.S.H. Vles (Accepted). Fetal asphyctic preconditioning protects against perinatal asphyxia-induced behavioral consequences in adulthood. Behavioral Brain Research.

E. Strackx, D.L.A. Van den Hove, L.J. Zimmermann, H.W.M. Steinbusch, C.E. Blanco, J.S.H. Vles and A.W.D. Gavilanes (Submitted.) Fetal asphyctic preconditioning protects against perinatal-asphyxia induced apoptosis and astrogliosis.

E.Strackx, M. Gantert, I.A.J. van Kooten, R. Rieke, H. Hürter, M.A.M. Lemmens, H.W.M. Steinbusch, L.J.I. Zimmermann, J.S.H. Vles, Y. Garnier, A.W.D. Gavilanes and B.W. Kramer (Submitted). Increased cerebellar granule cell number in sheep following prenatal injection of lipopolysaccharide.

\footnotetext{
Abstracts

E. Strackx, D.L.A. Van den Hove, H.P. Steinbusch, H.W.M. Steinbusch, J.S.H. Vles, C.E. Blanco and A.W.D. Gavilanes (2007) Age-related differences in the serotonergic system of the dorsal raphe nucleus after fetal asphyxia. Euron PhD student meeting, Brussels (Poster)

E. Strackx, D.L.A. Van den Hove, H.P. Steinbusch, H.W.M. Steinbusch, J.S.H. Vles, C.E. Blanco and A.W.D. Gavilanes (2007) Fetal asphyxia affects the nigrostriatal dopaminergic system and motor behavior in 6-month-old rats. Research Day of School of Mental Health and Neuroscience, Maastricht (Poster)

E. Strackx, D.L.A. Van den Hove, H.P. Steinbusch, H.W.M. Steinbusch, J.S.H. Vles, B.W. Kramer and A.W.D. Gavilanes (2008) Maturation-related differences in the response to endotoxin-induced chorioamnionitis in the ovine fetal brain.
} 
Research Day of School of Mental Health and Neuroscience, Maastricht (Poster)

E. Strackx, M. Gantert, D.L.A. Van den Hove, H.P. Steinbusch, Y. Garnier, L.J. Zimmermann, B.W. Kramer, A.W.D. Gavilanes and J.S.H. Vles (2008) Maturation-related differences in the response of brain, cerebellum and spinal cord to antanatal inflammation in fetal sheep. Pediatric Academic Societies' meeting, Hawaï (Poster)

B. Zoer, E. Strackx, L. Cobeño, J.S.H. Vles, E. Villamor and A.W.D. Gavilanes (2008) Reactivity of neonatal carotid arteries in a model of preconditioning induced by fetal asphyxia. Pediatrics Academic Societies' Meeting, Hawaï (Poster)

E. Strackx, D.L.A. Van den Hove, H.P. Steinbusch, C.E. Blanco, H.W.M. Steinbusch, J.S.H. Vles and A.W.D. Gavilanes (2008) Fetal asphyxia protects against severe perinatal asphyxia-induced apoptosis in a new rat model for hypoxicischemic preconditioning. Euron PhD Student Meeting, Aachen /Oral presentation)

E. Strackx, D.L.A. Van den Hove, H.P. Steinbusch, C.E. Blanco, H.W.M. Steinbusch, J.S.H. Vles and A.W.D. Gavilanes (2008) Fetal asphyxia protects against severe perinatal asphyxia-induced apoptotic cell death in a new rat model for hypoxic-ischemic preconditioning. Society for Neuroscience, Washington (Poster)

E. Strackx, D.L.A. Van den Hove, H.P. Steinbusch, C.E. Blanco, H.W.M. Steinbusch, J.S.H. Vles and A.W.D. Gavilanes (2009) Behavioral consequences of fetal asphyctic preconditioning in the adult rat. Research Day of School of Mental Health and Neuroscience, Maastricht (Poster)

E. Strackx, D.L.A. Van den Hove, H.P. Steinbusch, H.W.M. Steinbusch, C.E. Blanco, J.S.H. Vles and A.W.D. Gavilanes (2009) No behavioral changes in the adult rat in a new model for fetal asphyctic preconditioning. Pediatric Academic Societies' Meeting, Baltimore (Poster) 
Author

D.L.A. Van den Hove, PhD

H.P. Steinbusch

H.W.M. Steinbusch, Prof.

J.S.H. Vles, Prof.

C.E. Blanco, Prof

A.W.D. Gavilanes, PhD, MD.

J. Prickaerts, PhD

B. Zoer, MSC

E. Villamor, PhD, MD

K.E.M. Cox

J.R.A. Van Ekkendonk

L.J.I. Zimmermann, Prof.

\section{Affiliations of the co-authors}

Affiliation

School for Mental Health and Neuroscience Maastricht University, The Netherlands EURON

School for Mental Health and Neuroscience Maastricht University, The Netherlands EURON

School for Mental Health and Neuroscience Maastricht University, The Netherlands EURON

Department of Child Neurology Maastricht University Medical Center Maastricht, The Netherlands

Department of Pediatrics Maastricht University Medical Center Maastricht, The Netherlands

Department of Pediatrics Maastricht University Medical Center Maastricht, The Netherlands School for Mental Health and Neuroscience Maastricht University, The Netherlands EURON

Department of Pediatrics Maastricht University Medical Center Maastricht, The Netherlands Department of Pediatrics Maastricht University Medical Center Maastricht, The Netherlands

School for Mental Health and Neuroscience Maastricht University, The Netherlands EURON

School for Mental Health and Neuroscience Maastricht University, The Netherlands EURON

Department of Pediatrics Maastricht University Medical Center Maastricht, The Netherlands 
